\title{
High-energy operator product expansion at sub-eikonal level
}

\author{
Giovanni Antonio Chirilli \\ Institut für Theoretische Physik, Universität Regensburg, \\ Universitätsstrasse 31, D-93040 Regensburg, Germany \\ E-mail: giovanni.chirilli@gmail.com
}

ABSTRACT: The high energy Operator Product Expansion for the product of two electromagnetic currents is extended to the sub-eikonal level in a rigorous way. I calculate the impact factors for polarized and unpolarized structure functions, define new distribution functions, and derive the evolution equations for unpolarized and polarized structure functions in the flavor singlet and non-singlet case.

KEYwORDS: Perturbative QCD, Resummation

ArXiv EPrint: 2101.12744 


\section{Contents}

1 Introduction 1

2 Operator Product Expansion at high-energy 3

3 Sub-eikonal corrections to the high-energy OPE $\quad 8$

3.1 Quark propagator in the background of gluon 8

$\begin{array}{ll}3.2 & \text { Quark propagator in the background of quark fields } \\ \end{array}$

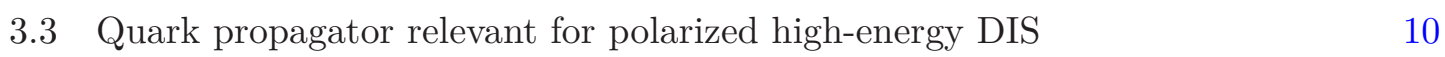

4 Operator Product Expansion with sub-eikonal terms 11

$\begin{array}{lll}4.1 & \text { OPE with quark sub-eikonal terms } & 11\end{array}$

$\begin{array}{lll}\text { 4.2 OPE with gluon sub-eikonal terms } & 15\end{array}$

$\begin{array}{lll}4.3 & \text { OPE with sub-eikonal corrections: flavor singlet } & 16\end{array}$

$\begin{array}{lll}\text { 4.4 OPE with sub-eikonal contributions: flavor non-singlet } & 17\end{array}$

$\begin{array}{llr}5 & \text { Parametrization of the forward matrix elements } & 18\end{array}$

6 Evolution equation of sub-eikonal corrections $\quad 21$

6.1 Diagrams with $\hat{Q}_{1 x}$ and $\hat{Q}_{5 x}$ quantum 22

6.2 Diagrams with $\hat{\tilde{Q}}_{1 x}$ and $\tilde{\tilde{Q}}_{5 x}$ quantum 24

$\begin{array}{lll}6.3 & \text { BK-type diagrams } & 25\end{array}$

6.3.1 BK-type diagrams for $Q_{1 x}$ and $Q_{5 x} \quad 26$

6.3.2 BK-type diagrams with $\tilde{Q}_{1 x}, \tilde{Q}_{5 x}, \mathcal{F}_{x} \quad 26$

$\begin{array}{ll}6.4 & \text { Evolution equation with } \epsilon^{i j} F_{i j} \text { quantum }\end{array}$

6.5 Diagrams with quark-to-gluon propagator 34

6.5.1 Quark-to-gluon propagator 34

$\begin{array}{ll}\text { 6.5.2 Operators definition } & 37\end{array}$

6.5.3 Quark-to-gluon diagrams for $\tilde{Q}_{1 x}$ and $\tilde{Q}_{5 x} \quad 37$

6.5.4 Quark-to-gluon diagrams for $\hat{Q}_{1 x}$ and $\hat{Q}_{5 x} \quad 41$

7 Summary of evolution equations $\quad \mathbf{4 5}$

$7.1 Q_{1 x}$ and $\tilde{Q}_{1 x}$ operators 46

$7.2 Q_{5 x}, \tilde{Q}_{5 x}$, and $\mathcal{F}_{x}$ operators: flavor singlet 47

$7.3 Q_{5 x}, \tilde{Q}_{5 x}$, and $\mathcal{F}_{x}$ operators: flavor non-singlet $\quad 50$

8 Conclusions $\quad 51$

$\begin{array}{ll}\text { A Notation } & 53\end{array}$ 
B Quark and gluon propagators with sub-eikonal corrections 54

B.1 Quark propagator in the background of gluon fields 55

B.2 Gluon propagator in the background of gluon field 59

B.3 Gluon propagator in the background of quark field 60

$\begin{array}{ll}\text { C Evaluation of matrix elements } & \mathbf{6 0}\end{array}$

$\begin{array}{lll}\text { C.1 Matrix element with } \hat{\mathcal{O}}_{1} \text { operator } & 61\end{array}$

$\begin{array}{lll}\text { C.2 Matrix element with } \hat{\mathcal{O}}_{j} \text { operator } & 62\end{array}$

C.3 Matrix element with $\hat{\mathcal{O}}_{\bullet *}$ operator 63

$\begin{array}{lll}\text { C.4 Quark propagator for } g_{1} \text { structure function } & 63\end{array}$

D Derivation of the OPE with quark-sub-eikonal corrections $\quad 64$

$\begin{array}{ll}\text { E Coefficients } I_{1}^{\mu \nu} \text { and } I_{5}^{\mu \nu} & 65\end{array}$

$\begin{array}{lll}\text { F Evolution equation for operators } \mathcal{Q}_{1 x} \text { and } \mathcal{Q}_{5 x} & 66\end{array}$

$\begin{array}{lll}\text { F.1 Diagrams with } \mathcal{Q}_{1 x} \text { and } \mathcal{Q}_{5 x} \text { quantum } & 66\end{array}$

$\begin{array}{lll}\text { F.2 Diagrams quark-to-gluon for } \mathcal{Q}_{1 x} \text { and } \mathcal{Q}_{5 x} & 69\end{array}$

$\begin{array}{lll}\text { F.2.1 Quark-to-gluon diagrams for } \mathcal{Q}_{1 x} & 72\end{array}$

$\begin{array}{lll}\text { F.2.2 Quark-to-gluon diagrams for } \mathcal{Q}_{5 x} & 74\end{array}$

F.3 Evolution equation with operators in the adjoint representation $\quad 75$

\section{Introduction}

In view of future collider experiments, in which hadronic matter will be probed at unprecedented kinematic regimes, there has been, in recent years, an ever growth of interest in understanding the fundamental properties of hadrons, spin and mass, from their constituents, quarks, and gluons [1-6, 9-13].

At high energy (Regge limit) scattering amplitudes are dominated by gluon dynamics, in particular, the cross section of Deep Inelastic Scattering (DIS) processes, is dominated, in the unpolarized case, by the gluon structure function. Within the Leading Log Approximation (LLA), the resummation of log of energy through BFKL [14, 15] formalism predicts a steep rise of the DIS cross-section that has been observed experimentally at HERA experiments $[16,17]$.

In perturbative quantum chromodynamics (pQCD) a standard technique to study the asymptotic behavior of cross-sections is the operator product expansions (OPE). In deep inelastic scattering (DIS), in the Bjorken limit, the $T$-product of two electromagnetic currents is expanded in terms of coefficient functions, perturbatively calculable, and matrix elements of non-local operators that encode the non perturbative information of the process. Evolution equations of the non-local matrix elements with respect to the factorization scale $\mu_{f}$ provide information on the scaling behavior of the parton distributions [18]. 
About twenty-five years ago, the high-energy Operator Product Expansion was introduced for the first time as a method to study the asymptotic behavior of high-energy structure functions from a gauge-invariant formalism [19]. Since then, the high-energy OPE proved to be a successful method to systematically study high-energy scattering amplitudes [20-23]. Within the high-energy OPE the scattering amplitude is factorized in rapidity space in coefficient functions (also called impact factors) and matrix elements of Wilson lines. The evolution equation of Wilson lines with respect to the rapidity parameter provides the energy dependence of the amplitude: each step in rapidity generates a new Wilson line at a different point in impact parameter space thus obtaining the non-linear Balitsky-JIMWLK evolution equation [19, 24-26] which in the dipole approximation takes the form of the Balitsky-Kovchegov equation [27, 28] (see refs. [29, 30] for a review).

Contrary to the OPE in the Bjorken limit, in the high-energy OPE the coefficient functions and the matrix elements both receive perturbative and non-perturbative contributions. The latter, however, are screened by the saturation scale, which being much larger than the scale of the confinement region, justifies the applicability of perturbative methods.

The unpolarized DIS structure functions at small-x are known at next-to-leading order (NLO) $[22,23]$ (see also [32]) in $\alpha_{s}$ and at next-leading-logarithmic accuracy [20, 31]. The NLO pomeron intercept, through which the log of energy are resummed at next-to-leading log approximation (NLL), has been calculated long ago [31] and later confirmed through the linearizion of the NLO Balitsky-Kovchegov equation [20]. Moreover, the pomeron residue, which is related to the impact factor is now available in an analytic form in coordinate and momentum space at NLO $[22,23]$. These two ingredients, the NLO pomeron intercept and the NLO pomeron residue allow one to study the behavior of the small-x structure function at NLO accuracy in $\alpha_{s}$ and at NLL approximation. A first fit of the dipole model to HERA data using the full NLO impact factor and Balitsky-Kovchegov evolution has been performed in ref. [33].

Unlike the unpolarized one, the behavior of the polarized DIS structure functions at small-x is not yet well understood. In the polarized case, the pomeron intercept receives an extra complication due to the presence of double logarithm of energy $\alpha_{s} \ln ^{2} x_{B}^{-1}[36$, 37]. Such double logarithms of energy are also present in the unpolarized quark structure function $[34,35]$, although they are suppressed by one power of energy with respect to the gluon one. In the polarized case, instead, quark structure functions are not suppressed thus their behavior at small- $x_{B}$ is as relevant as the gluon structure functions.

What makes the study of polarized structure functions even more challenging is the fact that, the double logarithm of energy, $\alpha_{s} \ln ^{2} x_{B}^{-1}$, cannot be reached from the LO anomalous dimension of twist two operators. Indeed, if on one side, the relation at the non-physical point $n=1$ ( $n$ being the moment index), between the BFKL and DGLAP was established through the anomalous dimension of twist two operators [38, 39], on the other, such relation cannot be easily obtained in the polarized case due to the presence of double log of energy contributions which are absent in the LO [40] and NLO polarized splitting functions [41]. However, the double log of energy contribution, $\alpha_{s} \ln ^{2} x_{B}^{-1}$, appears at there-loop polarized splitting function [42-45] and the result is in agreement with the one originally found in refs. [36, 37]. 
The plan is to bring the knowledge of the unpolarized and polarized small-x structure function at the same level. To this end, first we need to extend the high-energy OPE of the $T$-product of two-electromagnetic currents to include terms that are not symmetric in the exchange of the two Lorentz indexes of the DIS hadronic tensor. Indeed, in the polarized DIS both the leptonic tensor and the hadronic one have to be antisymmetric. To obtain antisymmetric contributions in the high-energy OPE, it is necessary to relax the eikonal approximation and allow sub-eikonal terms to enter into the game. As we will show in the subsequent sections, the OPE with sub-eikonal contributions will be given in terms of new operators and the task will be to calculate their evolution equations. An important ingredient to perform the high-energy OPE with sub-eikonal corrections is the quark and gluon propagators with sub-eikonal corrections. In ref. [46] such corrections have been all calculated as deviation from the shock-wave approximation: the corrections are suppressed by the large Lorentz boost parameter. Corrections to the eikonal approximation can be also included by assuming that the fields of the background shock-wave do not have the same transverse momenta, rather they are ordered. In this case the sub-eikonal corrections are included as light-cone expansion of the background shock-wave [47, 48]. However, these corrections will be irrelevant for our analysis since here we are interested in small-x dynamics rather than studying the overlapping kinematic regime where these corrections will play a central role.

In section 2 we will review the main idea behind the high-energy OPE in the unpolarized case. We will derive the leading order (LO) impact factor and the associated Wilson-line operators together with their evolution equations. This will serve as a smooth transition to the OPE at sub-eikonal level in section 3 where we will give a new expression (equivalent to the one obtained before in ref. [46]) of the quark propagator with sub-eikonal corrections in the background of gluon fields, and will identify the relevant sub-eikonal corrections which will be used, in section 4 , to calculate the impact factors for polarized and unpolarized structure functions. In section 5 we summarize the parametrization of the matrix elements of operators found through the OPE, and identify 10 new distribution functions. In section 6 we will derive the evolution equations for the operators associated to the sub-eikonal impact factors using the propagators calculated in previous work, ref. [46]. In the same section we will calculate, for the first time, the quark-to-gluon propagator and use it to calculate diagrams that have not been calculated before in the contest of spin at small-x. Summary of the evolution equations for singlet and non-singlet case will be presented in section 7 . In the last section we will summarize our findings and compare them with other results that have been obtained in the same direction. We will argue that the result we obtained although agree in some limiting case, they actually differ from the ones calculated in refs. $[5,7,8]$ because of the way the quark and gluon operators are treated under one loop evolution.

\section{Operator Product Expansion at high-energy}

Before calculating the sub-eikonal corrections to the high-energy OPE for DIS, let us first briefly review the high-energy OPE in the eikonal approximation. 
The inclusive differential DIS cross-section in the laboratory frame for detecting the final lepton in the solid angle $d \Omega$ with final energy within $\left[E^{\prime}, E^{\prime}+d E^{\prime}\right]$ is

$$
\frac{d^{2} \sigma}{d \Omega d E^{\prime}}=\frac{\alpha^{2}}{M q^{4}} \frac{E^{\prime}}{E} L_{\mu \nu} W^{\mu \nu}
$$

Here the hadronic target of mass $M$ has momentum $P^{\mu}=p_{2}^{\mu}+\frac{M^{2}}{s} p_{1}^{\mu}$, and the virtual photon has momentum $q^{\mu}=p_{1}^{\mu}-x_{B} p_{2}^{\mu}$ with $p_{1}^{\mu}, p_{2}^{\mu}$ light-cone vectors such that $p_{1}^{\mu} p_{2 \mu}=\frac{s}{2}$ and $x_{B}=\frac{-q^{2}}{s} \ll 1$. The leptonic tensor is denoted by $L^{\mu \nu}$ and the hadronic one by $W^{\mu \nu}$.

In strong and electromagnetic interactions parity is conserved, thus the hadronic tensor can be expanded in terms of the unpolarized structure functions $F_{1}$ and $F_{2}$ and the polarized structure functions $g_{1}$ and $g_{2}$

$$
\begin{aligned}
W_{\mu \nu}= & \left(-g_{\mu \nu}+\frac{q_{\mu} q_{\nu}}{q^{2}}\right) F_{1}\left(x, Q^{2}\right)+\left(P_{\mu}-q_{\mu} \frac{q \cdot P}{q^{2}}\right)\left(P_{\nu}-q_{\nu} \frac{q \cdot P}{q^{2}}\right) \frac{F_{2}\left(x, Q^{2}\right)}{P \cdot q} \\
& +i \varepsilon_{\mu \nu \lambda \sigma} q^{\lambda} S^{\sigma} \frac{M}{P \cdot q} g_{1}\left(x, Q^{2}\right)+i \varepsilon_{\mu \nu \lambda \sigma} q^{\lambda}\left(S^{\sigma}-P^{\sigma} \frac{q \cdot S}{q \cdot P}\right) \frac{M}{P \cdot q} g_{2}\left(x, Q^{2}\right)
\end{aligned}
$$

where $S^{\mu}$ is the spin of the target that satisfies $S^{2}=-1$ and $S \cdot P=0$.

To extract the polarized structure functions $g_{1}$ and $g_{2}$, we need the antisymmetric part of the leptonic tensor. This means that both the incoming lepton and the hadronic target have to be polarized.

With the help of the optical theorem, the hadronic tensor is related to the imaginary part of the Fourier transform of the $T$-product of two electromagnetic currents

$$
W_{\mu \nu}=\frac{1}{\pi} \operatorname{Im} T_{\mu \nu} .
$$

where

$$
T_{\mu \nu}=i \int d^{4} x e^{i q \cdot x}\left\langle P, S\left|\mathrm{~T}\left\{j_{\mu}(x) j_{\nu}(0)\right\}\right| P, S\right\rangle
$$

To study the polarized structure functions $g_{1}$ and $g_{2}$ at high-energy we need to extract the antisymmetric part of the $T_{\mu \nu}$ tensor. This is what we will do in the next section.

The T-product of two electromagnetic currents is considered in the background of gluon field. As we will see later, in order to calculate sub-eikonal corrections it will be necessary to consider the OPE in the background of gluons and quarks as well. However for the moment we consider a background made only of gluons.

In the spectator frame the background field reduces to a shock wave (see appendix A and [29] for review), and the virtual photon, which mediates the interactions between the lepton and the nucleon (or nucleus) in DIS processes, splits into a quark anti-quark pair long before the interaction with the target. In the eikonal approximation the propagation of the quark anti-quark pair in the shock wave background, reduces to two infinite Wilson lines. Although with less probability, the virtual photon may fluctuate in a quark, an anti-quark and a gluon before interacting with the target. In this case, the number of Wilson lines increases. Consequently, at high energy, the T-product of two electromagnetic currents is 
expanded in terms of infinite Wilson lines as

$$
\begin{aligned}
& T\left\{\overline{\hat{\psi}}(x) \gamma^{\mu} \hat{\psi}(x) \overline{\hat{\psi}}(y) \gamma^{\nu} \hat{\psi}(y)\right\} \\
& =\int d^{2} z_{1} d^{2} z_{2} I_{\mathrm{LO}}^{\mu \nu}\left(z_{1}, z_{2}, x, y\right) \operatorname{Tr}\left\{\hat{U}_{z_{1}}^{\eta} \hat{U}_{z_{2}}^{\dagger \eta}\right\} \\
& \quad+\int d^{2} z_{1} d^{2} z_{2} d^{2} z_{3} I_{\mathrm{NLO}}^{\mu \nu}\left(z_{1}, z_{2}, z_{3}, x, y\right)\left[\operatorname{tr}\left\{\hat{U}_{z_{1}}^{\eta} \hat{U}_{z_{3}}^{\dagger \eta}\right\} \operatorname{tr}\left\{\hat{U}_{z_{3}}^{\eta} \hat{U}_{z_{2}}^{\dagger \eta}\right\}-N_{c} \operatorname{tr}\left\{\hat{U}_{z_{1}}^{\eta} \hat{U}_{z_{2}}^{\dagger \eta}\right\}\right]+\cdots
\end{aligned}
$$

where with the symbol ^ over the fields we indicate operators, $U_{x}=\operatorname{Pexp}\left\{i g \int d x^{+} A^{-}\left(x^{+}+\right.\right.$ $\left.\left.x_{\perp}\right)\right\}$ is the Wilson line and $A^{-}$is the background gluon field. The first term of expansion (2.5), which is proportional to the LO impact factor $I_{\mathrm{LO}}$, corresponds to the probability for the virtual photon, to split in a quark-anti-quark pair. The second term, proportional to the NLO impact factor $I_{N L O}$, and which has been calculated in refs. [22, 23], corresponds to the probability that the virtual photon has to split in a quark-anti-quark and a gluon before scattering with the target. Here we use the light-cone variables $x^{ \pm}=\frac{x^{0} \pm x^{3}}{\sqrt{2}}$. In appendix A we provide further details on the notation used throughout this paper.

Let us see how to get expansion (2.5) in a bit more detail. We know that to get the DIS cross-section we need to evaluate the T-product of two electromagnetic currents in the nucleus or nucleon target state $|P, S\rangle$

$$
\left\langle P, S\left|\mathrm{~T}\left\{\overline{\hat{\psi}}(x) \gamma^{\mu} \hat{\psi}(x) \overline{\hat{\psi}}(y) \gamma^{\nu} \hat{\psi}(y)\right\}\right| P, S\right\rangle
$$

Since we do not know exactly the proton or nucleus state in terms of quarks and gluons, we are forced to make approximations which are suitable for the kinematic regime under consideration. As anticipated before, at high-energy (Regge limit), the target state, made by quarks and gluons, represents the background field which, in the conveniently chosen spectator frame, shrinks into a shock wave. In first (eikonal) approximation the shock-wave is made only by gluon field. In light of these considerations we have

$$
\left\langle P, S\left|\mathrm{~T}\left\{\overline{\hat{\psi}}(x) \gamma^{\mu} \hat{\psi}(x) \overline{\hat{\psi}}(y) \gamma^{\nu} \hat{\psi}(y)\right\}\right| P, S\right\rangle \quad \rightarrow \quad\left\langle\mathrm{T}\left\{\bar{\psi}(x) \gamma^{\mu} \psi(x) \bar{\psi}(y) \gamma^{\nu} \psi(y)\right\}\right\rangle_{A}
$$

where the subscript $A$ indicates that the matrix element is evaluated in the background of gluon field generated by the target. The transition from the l.h.s. to the r.h.s. of eq. (2.7) is similar to the analysis that one performs in the usual local OPE in which one considers the target in terms of its partonic content, quarks or gluons. In ref. [18] it was shown that the local OPE can be reformulated in terms of non-local operators using the background field method. It turns out that also at high-energy (Regge) limit it is more convenient to perform the OPE analysis using the background field method [19]. Once the relevant operators are obtained, and we will see they are infinite Wilson lines, they will be evaluated in the target state again. We now perform functional integration over the spinor fields. Considering only the fully connected diagrams, we have (see figure 1)

$$
\left\langle\mathrm{T}\left\{\bar{\psi}(x) \gamma^{\mu} \psi(x) \bar{\psi}(y) \gamma^{\nu} \psi(y)\right\}\right\rangle_{A}=\operatorname{tr}\left\{\left\langle x\left|\frac{1}{\not P+i \epsilon}\right| y\right\rangle \gamma^{\nu}\left\langle y\left|\frac{1}{\not P+i \epsilon}\right| x\right\rangle \gamma^{\mu}\right\}
$$

where $P^{\mu}=p^{\mu}+g A^{\mu}$ and $A^{\mu}$ is the background gluon field. In eq. (2.8) we have used the Schwinger notation for the quark propagator which in the eikonal approximation is

$$
\langle\mathrm{T} \psi(x) \bar{\psi}(y)\rangle^{x^{+}>0>y^{+}} \int d^{4} z \delta\left(z^{+}\right) \frac{\not x-\not z}{2 \pi^{2}(x-z)^{4}} \not \phi_{2} U_{z} \frac{\not \neq-\not y}{2 \pi^{2}(y-z)^{4}}
$$




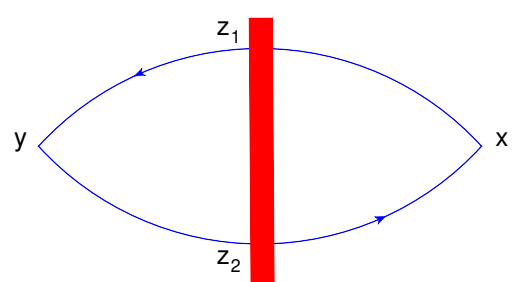

Figure 1. Diagram for the LO impact factor in the eikonal approximation. As usual, we will indicate in blue the quantum field and in red the classical background ones.

with $x^{\mu} \gamma_{\mu}=\not x$. In figure 1 the blue lines represent the quark and anti-quark fields, while the red band represents the background shock-wave field.

Using propagator (2.9) in eq. (2.8) arrive at

$$
\begin{aligned}
& \left\langle\mathrm{T}\left\{\bar{\psi}(x) \gamma^{\mu} \psi(x) \bar{\psi}(y) \gamma^{\nu} \psi(y)\right\}\right\rangle_{A} \\
& \stackrel{x^{+}>0>y^{+}}{=}-\frac{1}{4 \pi^{6}\left(x_{*} y_{*}\right)^{4}} \int d^{2} z_{1} d^{2} z_{2}\left\langle\operatorname{Tr}\left\{U\left(z_{1 \perp}\right) U^{\dagger}\left(z_{2 \perp}\right)\right\}\right\rangle_{A} \\
& \times \frac{\operatorname{tr}\left\{\left(\not x-\not \phi_{1}\right) \not p_{2}\left(\not y-\not \not_{1}\right) \gamma^{\nu}\left(\not y-\not \not_{2}\right) \not p_{2}\left(\not x-\not \not_{2}\right) \gamma^{\mu}\right\}}{\left[\mathcal{Z}_{1}+i \epsilon\right]^{3}\left[\mathcal{Z}_{2}+i \epsilon\right]^{3}}+\ldots
\end{aligned}
$$

where the $\ldots$ stand for higher order corrections in $\alpha_{s}$ or sub-eikonal corrections. In eq. (2.10) we defined

$$
\mathcal{Z}_{i} \equiv \frac{\left(x-z_{i}\right)_{\perp}^{2}}{x_{*}}-\frac{\left(y-z_{i}\right)_{\perp}^{2}}{y_{*}}-\frac{4}{s}\left(x_{\bullet}-y_{\bullet}\right)
$$

where we use the short-hand notation (see appendix A for further details on the notations used through out the paper)

$$
\begin{aligned}
& p_{1}^{\mu} x_{\mu}=x_{\bullet}=\sqrt{\frac{s}{2}} x^{-}=\sqrt{\frac{s}{2}} x_{+}, \\
& p_{2}^{\mu} x_{\mu}=x_{*}=\sqrt{\frac{s}{2}} x^{+}=\sqrt{\frac{s}{2}} x_{-} .
\end{aligned}
$$

Using the identity

$$
\begin{aligned}
\operatorname{tr}\left\{\left(\not x-\not \not_{1}\right) \not p_{2}\left(\not y-\not \not_{1}\right) \gamma^{\nu}\left(\not y-\not \not_{2}\right) \not p_{2}\left(\not x-\not \not_{2}\right) \gamma^{\mu}\right\} \\
=4 x_{*}^{2} y_{*}^{2} \frac{\partial^{2}}{\partial x_{\mu} \partial y_{\nu}}\left(-\mathcal{Z}_{1} \mathcal{Z}_{2}+\frac{z_{12 \perp}^{2}}{x_{*} y_{*}}(x-y)^{2}\right)
\end{aligned}
$$

we define the LO impact factor as

$$
\mathcal{I}_{L O}^{\mu \nu}\left(z_{1 \perp}, z_{2 \perp} ; x, y\right) \equiv \frac{1}{\pi^{6}\left(x_{*} y_{*}\right)^{2}} \frac{\left(\mathcal{Z}_{1}+i \epsilon\right)^{-3}}{\left(\mathcal{Z}_{2}+i \epsilon\right)^{3}} \frac{\partial^{2}}{\partial x_{\mu} \partial y_{\nu}}\left(\mathcal{Z}_{1} \mathcal{Z}_{2}-\frac{z_{12 \perp}^{2}}{x_{*} y_{*}}(x-y)^{2}\right)
$$

and the high-energy OPE takes the form

$$
\mathrm{T}\left\{\overline{\hat{\psi}}(x) \gamma^{\mu} \hat{\psi}(x) \overline{\hat{\psi}}(y) \gamma^{\nu} \hat{\psi}(y)\right\}=\int d z_{1} d z_{2} \mathcal{I}_{L O}^{\mu \nu}\left(z_{1 \perp}, z_{2 \perp} ; x, y\right) \operatorname{Tr}\left\{\hat{U}\left(z_{1 \perp}\right) \hat{U}^{\dagger}\left(z_{2 \perp}\right)\right\}+\ldots
$$




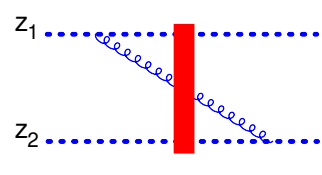

a)

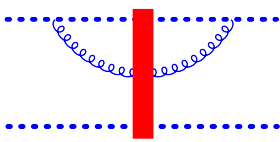

c)

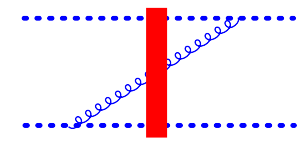

b)

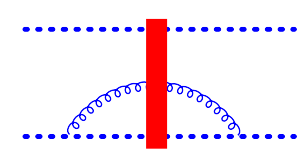

d)

Figure 2. Sample of diagrams (real ones) for the LO BK equation.

Eq. (2.16) tell us that in the high-energy limit the operators standing to the left of the equation are approximated by the ones standing to the right.

The LO impact factor enjoys two nice properties: electromagnetic gauge invariance

$$
\frac{\partial}{\partial x^{\mu}} \mathcal{I}_{L O}^{\mu \nu}\left(z_{1 \perp}, z_{2 \perp} ; x, y\right)=0
$$

and Möbius $\operatorname{SL}(2, C)$ conformal invariance

$$
\int d^{2} z_{1} d^{2} z_{2} \mathcal{I}_{L O}^{\mu \nu}\left(z_{1 \perp}, z_{2 \perp} ; x, y\right) \stackrel{\mathrm{inv}_{\dot{1}}}{\longrightarrow} \int d^{2} z_{1} d^{2} z_{2} \mathcal{I}_{L O}^{\mu \nu}\left(z_{1 \perp}, z_{2 \perp} ; x, y\right)
$$

where the symbol $\stackrel{\text { inv }}{\longrightarrow}$ means that we perform the inversion transformation $\frac{x^{\mu}}{x^{2}}$ to all coordinates.

If we try to calculate one loop correction either to the coefficient function (the impact factor) or to the matrix element of the Wilson-line operators, we will find divergences which are identified by rapidity divergences as a remnant of the fact that the parameter we use to discriminate between background (or classical) field from the quantum field is indeed the rapidity. As explained in the Introduction, at high-energy fields are ordered in their rapidity.

The rapidity divergences we just mentioned represent the log of energy which are usually resummed through an evolution equation. The easiest way to identify such log of energy is to consider one loop correction to the matrix element of Wilson lines, rather then to the coefficient function. In refs. $[22,23]$ a one loop correction to the coefficient function (NLO impact factor) was calculated, and it was shown that the BK evolution equation can also be obtained from the one loop correction to the coefficient function (impact factor). One may observe the similarity with the non-local OPE where DGLAP evolution kernel can be obtained either from the one loop evolution of the non-local operator or from the NLO coefficient function [18].

The evolution of the $\operatorname{Tr}\left\{\hat{U}\left(z_{\perp}\right) \hat{U}^{\dagger}\left(z_{\perp}^{\prime}\right)\right\}$ with respect to rapidity is the BK equation. The diagrams (except the virtual ones) contributing to the kernel of the evolution equation are given in figure 2. The evolution equation is [19]

$$
\begin{aligned}
\left\langle\operatorname{Tr}\left\{U_{z_{1}} U_{z_{2}}^{\dagger}\right\}\right\rangle= & \frac{\alpha_{s}}{2 \pi} \int \frac{d \alpha}{\alpha} \int d^{2} z \frac{\left(z_{1}-z_{2}\right)^{2}}{\left(z_{1}-z\right)^{2}\left(z_{2}-z\right)^{2}} \\
& \times\left[\operatorname{Tr}\left\{U_{z_{1}} U_{z}^{\dagger}\right\} \operatorname{Tr}\left\{U_{z} U_{z_{2}}^{\dagger}\right\}-N_{c} \operatorname{Tr}\left\{U_{z_{1}} U_{z_{2}}^{\dagger}\right\}\right] .
\end{aligned}
$$


The evolution equation is obtained in the background fileld method by integrating over the infinitesimal step in rapidity where $\int_{\eta_{2}}^{\eta_{1}} \frac{d \alpha}{\alpha} \rightarrow \Delta \eta$. Taking derivative with respect to rapidity we get the evolution equation

$$
\frac{d}{d \eta} \hat{\mathcal{U}}_{z_{1} z_{2}}^{\eta}=\frac{\alpha_{s} N_{c}}{2 \pi} \int d^{2} z \frac{\left(z_{1}-z_{2}\right)^{2}}{\left(z_{1}-z\right)^{2}\left(z_{2}-z\right)^{2}}\left[\hat{\mathcal{U}}_{z_{1} z}^{\eta}+\hat{\mathcal{U}}_{z z_{2}}^{\eta}-\hat{\mathcal{U}}_{z_{1} z_{2}}^{\eta}-\hat{\mathcal{U}}_{z_{1}}^{\eta} \hat{\mathcal{U}}_{z z_{2}}^{\eta}\right]
$$

where we indicate the rapidity dependence of the operators by the subscript $\eta$ and we defined the operator

$$
\hat{\mathcal{U}}\left(x_{\perp}, y_{\perp}\right)=1-\frac{1}{N_{c}} \operatorname{Tr}\left\{\hat{U}\left(x_{\perp}\right) \hat{U}^{\dagger}\left(y_{\perp}\right)\right\}
$$

Equation (2.20) is the Balitsky evolution equation. The operator we started with, $\operatorname{Tr}\left\{\hat{U}_{z_{1}} \hat{U}_{z_{2}}^{\dagger}\right\}$, after one loop evolution became a sum of two operators. One is clearly the same as the original one (before evolution), the other one, $\operatorname{Tr}\left\{\hat{U}_{z_{1}} \hat{U}_{z}^{\dagger}\right\} \operatorname{Tr}\left\{\hat{U}_{z} \hat{U}_{z_{2}}^{\dagger}\right\}$ is a new operator. To solve the evolution equation one should, in principle, find the evolution equation of the new operator as well. However, this will actually generate a further new operator. This process generates a hierarchy of evolution equations known as Balitsky-hierarchy. It is also known that the Balitsky-hierarchy is equivalent to the JIMWLK formalism [24-26] and for this reason, in the literature, they are mentioned together as B-JIMWLK equation.

In the large $N_{c}$ approximation, the matrix element of the new operator factorizes into a product of operators equal to the one we started with, thus breaking the Balitsky-hierarchy to the non-linear Balitsky-Kovchegov [19, 27, 28] equation

$$
\frac{d}{d \eta}\left\langle\mathcal{U}_{z_{1} z_{2}}^{\eta}\right\rangle=\frac{\alpha_{s} N_{c}}{2 \pi} \int \frac{d^{2} z\left(z_{1}-z_{2}\right)^{2}}{\left(z_{1}-z\right)^{2}\left(z_{2}-z\right)^{2}}\left[\left\langle\mathcal{U}_{z_{1} z}^{\eta}\right\rangle+\left\langle\mathcal{U}_{z z_{2}}^{\eta}\right\rangle-\left\langle\mathcal{U}_{z_{1} z_{2}}^{\eta}\right\rangle-\left\langle\mathcal{U}_{z_{1}}^{\eta}\right\rangle\left\langle\mathcal{U}_{z z_{2}}^{\eta}\right\rangle\right]
$$

If sub-eikonal corrections are included, as we will see, we will obtain new evolution equations which will generate different type of new operators.

\section{Sub-eikonal corrections to the high-energy OPE}

Now we are going to include the sub-eikonal corrections to the high-energy OPE. To this end we need the quark propagator with sub-eikonal corrections which was calculated in ref. [46].

\subsection{Quark propagator in the background of gluon}

The quark propagator in the background of gluon field with sub-eikonal corrections was calculated in ref. [46]. In appendix B, we provide a new derivation of the quark propagator which confirms the one obtained in the previous publication [46]. In the same appendix, we also put the result in a new form which facilitates the analysis of the matrix elements with sub-eikonal corrections. The new form of the quark propagator in the background of 
gluon fields up to subeikonal terms is

$$
\begin{aligned}
& \left\langle x\left|\frac{i}{\hat{P}+i \epsilon}\right| y\right\rangle \\
& =\left[\int_{0}^{+\infty} \frac{d \alpha}{2 \alpha} \theta\left(x_{*}-y_{*}\right)-\int_{-\infty}^{0} \frac{d \alpha}{2 \alpha} \theta\left(y_{*}-x_{*}\right)\right] e^{-i \alpha\left(x_{\bullet}-y_{\bullet}\right)} \frac{1}{\alpha s} \\
& \quad \times\left\langle x_{\perp}\right| e^{-i \frac{\hat{p}_{\perp}^{2}}{\alpha s} x_{*}}\left\{\hat{\not p} \not p_{2}\left[x_{*}, y_{*}\right] \hat{\not p}+\hat{\not p} \not p_{2} \hat{\mathcal{O}}_{1}\left(p_{\perp} ; x_{*}, y_{*}\right) \hat{\not p}\right. \\
& \quad+\frac{i \alpha s}{2} \epsilon^{i j} \gamma^{5} \gamma_{i} \hat{\mathcal{O}}_{j}\left(p_{\perp} ; x_{*}, y_{*}\right)-\frac{1}{2} \not p_{2}\left[\hat{p}^{j}, \hat{\mathcal{O}}_{j}\left(p_{\perp} ; x_{*}, y_{*}\right)\right]-\frac{i}{2} \epsilon^{i j} \gamma^{5} \not p_{2}\left\{\hat{p}_{i}, \hat{\mathcal{O}}_{j}\left(p_{\perp} ; x_{*}, y_{*}\right)\right\} \\
& \left.\quad+\frac{i \alpha s}{2} \epsilon^{i j} \gamma^{5} \gamma_{j}\left\{p_{i}, \mathcal{O}_{\bullet *}\left(x_{*}, y_{*}\right)\right\}-\frac{1}{2} \not p_{2}\left[\hat{p}_{\perp}^{2}, \hat{\mathcal{O}}_{\bullet *}\left(x_{*}, y_{*}\right)\right]\right\} e^{i \frac{\hat{p}_{\perp}^{2}}{\alpha s} y_{*}}\left|y_{\perp}\right\rangle+O\left(\lambda^{-2}\right) .
\end{aligned}
$$

where operators $\mathcal{O}_{1}, \mathcal{O}_{j}$ and $\mathcal{O}_{\bullet *}$ are defined in eqs. (B.13), (B.15), and (B.17). This is a new expression (equivalent to the one obtained before in ref. [46]) of the quark propagator with sub-eikonal corrections in the background of gluon fields.

As it is shown in appendix C, from all the terms present in the quark propagator (3.1), the only one that contributes to the polarized $g_{1}$ structure function is

$$
\begin{aligned}
& \langle\mathrm{T}\{\psi(x) \bar{\psi}(y)\}\rangle_{\psi, \bar{\psi}} \\
& \stackrel{\substack{x_{*}>0>y_{*} \\
\ni}}{+\infty} \frac{d \alpha}{4 s \alpha^{3}} e^{-i \alpha\left(x_{\bullet}-y_{\bullet}\right)} \int d^{2} z\left\langle x_{\perp}\left|\not p e^{-i \frac{\hat{p}_{\perp}^{2}}{\alpha s} x_{*}}\right| z_{\perp}\right\rangle \\
& \quad \times i g \int_{-\infty}^{+\infty} d \frac{2}{s} z_{*} \not p_{2}\left[\infty p_{1}, z_{*}\right]_{z} \frac{1}{2} \sigma^{i j} F_{i j}\left(z_{*}, z_{\perp}\right)\left[z_{*},-\infty p_{1}\right]_{z}\left\langle z_{\perp}\left|\not p e^{i \frac{\hat{p}^{2}}{\alpha s} y_{*}}\right| y_{\perp}\right\rangle
\end{aligned}
$$

where we used $\sigma^{i j}=\frac{i}{2}\left(\gamma^{i} \gamma^{j}-\gamma^{j} \gamma^{i}\right)$, and the $\hbar$-inspired notation $d^{n} \alpha=\frac{d^{n} \alpha}{(2 \pi)^{n}}$, and we defined the gauge link at fixed transverse position $z_{\perp}$ as

$$
\operatorname{Pexp}\left\{i g \frac{2}{s} \int_{y_{*}}^{x_{*}} d z_{*} A_{\bullet}\left(z_{*}, z_{\perp}\right)\right\} \equiv\left[x_{*} p_{1}+z_{\perp}, y_{*} p_{1}+z_{\perp}\right] \equiv\left[x_{*}, y_{*}\right]_{z} .
$$

We will work again in coordinate space so, performing the Fourier transform of (3.2), we have

$$
\begin{aligned}
& \langle\operatorname{Tr}\{\psi(x) \bar{\psi}(y)\}\rangle_{\psi, \bar{\psi}} \\
& \stackrel{x_{*}>0>y_{*}}{\ni}-\frac{1}{16 \pi^{3} x_{*}^{2} y_{*}^{2}} \int \frac{d^{2} z}{[\mathcal{Z}+i \epsilon]^{2}}\left(\frac{2}{s} x_{*} \not \not_{1}+\not X_{\perp}\right) \frac{1}{s} \not p_{2} \gamma^{5} \mathcal{F}\left(z_{\perp}\right)\left(\frac{2}{s} x_{*} \not \not_{1}+Y_{\perp}\right)
\end{aligned}
$$

with

$$
\mathcal{Z} \equiv \frac{(x-z)_{\perp}^{2}}{x_{*}}-\frac{(y-z)_{\perp}^{2}}{y_{*}}-\frac{4}{s}\left(x_{\bullet}-y_{\bullet}\right)
$$

and where we have introduced the short-hand notation $X_{\perp}^{\mu}=(x-z)_{\perp}^{\mu}$ and similarly for $Y_{\perp}^{\mu}$ and defined, for later convenience, the operator

$$
\mathcal{F}\left(z_{\perp}\right) \equiv i g \frac{s}{2} \int_{-\infty}^{+\infty} d z_{*}\left[\infty p_{1}, z_{*}\right]_{z} \epsilon^{i j} F_{i j}\left(z_{*}, z_{1 \perp}\right)\left[z_{*},-\infty p_{1}\right]_{z} .
$$


We use the symbol $\ni$ to indicate that we are considering only one of the possible terms that make up the quark propagator. Moreover, the superscript $x_{*}>0>y_{*}$ indicates that we are considering only the contribution in which the quark goes through the shock-wave from positive values of light-cone coordinate, $x_{*}>0$, to negative ones, $y_{*}<0$.

Throughout this paper we use the convention $\gamma^{5}=i \gamma^{0} \gamma^{1} \gamma^{2} \gamma^{3}=-\frac{i}{4 !} \epsilon_{\mu \nu \rho \sigma}$ and $\epsilon^{0123}=$ 1. The two dimensional antisymmetric tensor is $p_{1 \mu} p_{2 \nu} \epsilon^{\mu \nu i j}=\frac{s}{2} \epsilon^{i j}$ where, as usual, Latin indexes take values 1,2 and $\epsilon^{12}=-\epsilon^{21}=1$.

\subsection{Quark propagator in the background of quark fields}

The quark propagator in the background of quark fields is [46]

$$
\begin{aligned}
\langle\mathrm{T}\{\psi(x) \bar{\psi}(y)\}\rangle_{\psi, \bar{\psi}} \stackrel{x_{*}>0>y_{*}}{\ni} g^{2} \int_{0}^{+\infty} \frac{d \alpha}{2 \alpha} \int_{-\infty}^{+\infty} d z_{*} \int_{-\infty}^{z_{*}} d z_{*}^{\prime} e^{-i \alpha\left(x_{\bullet}-y_{\bullet}\right)} \\
\times \frac{1}{\alpha^{4} s^{4}}\left\langle x_{\perp}\right| e^{-i \frac{\hat{p}_{\perp}^{2}}{\alpha s} x_{*}} \not p p_{2} \not p\left[\infty p_{1}, z_{*}\right] \gamma^{\mu} t^{a} \psi\left(z_{*}\right)\left(\delta_{\mu}^{\xi}-\frac{p_{2 \mu}}{p_{*}} p^{\xi}\right)\left[z_{*}, z_{*}^{\prime}\right]^{a b} \\
\times\left(g_{\xi \nu}-p_{\xi} \frac{p_{2 \nu}}{p_{*}}\right) \bar{\psi}\left(z_{*}^{\prime}\right) t^{b} \gamma^{\nu}\left[z_{*}^{\prime},-\infty p_{1}\right] \not p p_{2} \not p e^{i \frac{\hat{p}_{\perp}^{2}}{\alpha s} y_{*}}\left|y_{\perp}\right\rangle
\end{aligned}
$$

Performing the Fourier transform we have

$$
\begin{aligned}
& \langle\mathrm{T}\{\psi(x) \bar{\psi}(y)\}\rangle_{\psi, \bar{\psi}} \\
& \stackrel{x_{*}>0>y_{*}}{\ni}-\frac{g^{2}}{16 \pi^{3} x_{*}^{2} y_{*}^{2}} \int_{-\infty}^{+\infty} d z_{*} \int_{-\infty}^{z_{*}} d z_{*}^{\prime} \int \frac{d^{2} z}{\left(\mathcal{Z}^{2}+i \epsilon\right)^{2}}\left(\frac{2}{s} x_{*} \not p_{1}+X_{\perp}\right) \\
& \times\left[\infty p_{1}, z_{*}\right]_{z} t^{a}\left(\gamma_{\perp}^{\mu} \psi\left(z_{*}, x_{\perp}\right)\left[z_{*}, z_{*}^{\prime}\right]_{z}^{a b} \bar{\psi}\left(z_{*}^{\prime}, z_{\perp}\right) \gamma_{\mu}^{\perp}\right) t^{b}\left[z_{*}^{\prime},-\infty p_{1}\right]_{z}\left(\frac{2}{s} y_{*} \not p_{1}+Y_{\perp}\right)
\end{aligned}
$$

To highlight the structure of the propagator (3.8), let us define the following operator

$$
\begin{aligned}
& Q_{i j}^{\alpha \beta}\left(x_{\perp}\right) \\
& \equiv g^{2} \int_{-\infty}^{+\infty} d z_{*} \int_{-\infty}^{z_{*}} d z_{*}^{\prime}\left(\left[\infty p_{1}, z_{*}\right]_{x} t^{a} \psi^{\alpha}\left(z_{*}, x_{\perp}\right)\left[z_{*}, z_{*}^{\prime}\right]_{x}^{a b} \bar{\psi}^{\beta}\left(z_{*}^{\prime}, x_{\perp}\right) t^{b}\left[z_{*}^{\prime},-\infty p_{1}\right]_{x}\right)_{i j}
\end{aligned}
$$

where $\alpha$, and $\beta$ are spinor indexes, and $i, j$ are color indexes in the fundamental representation. Thus, the quark propagator (3.8) becomes

$$
\begin{aligned}
& \langle\mathrm{T}\{\psi(x) \bar{\psi}(y)\}\rangle_{\psi, \bar{\psi}} \\
& \stackrel{x_{*}>0>y_{*}}{\ni}-\frac{1}{16 \pi^{3} x_{*}^{2} y_{*}^{2}} \int \frac{d^{2} z}{(\mathcal{Z}+i \epsilon)^{2}}\left(\frac{2}{s} x_{*} \not p_{1}+\not X_{\perp}\right) \gamma_{\perp}^{\mu} Q\left(z_{\perp}\right) \gamma_{\mu}^{\perp}\left(\frac{2}{s} y_{*} \not p_{1}+Y_{\perp}\right) .
\end{aligned}
$$

\subsection{Quark propagator relevant for polarized high-energy DIS}

The quark propagator we need for polarized DIS at high-energy is the sum of the eikonal propagator (2.9), the sub-eikonal correction due to the background of gluon field (3.4), and the sub-eikonal correction due to the background made by quark fields eq. (3.10). Putting 


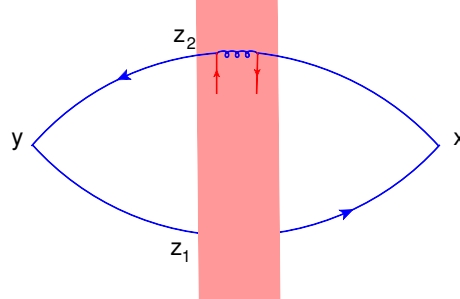

a)

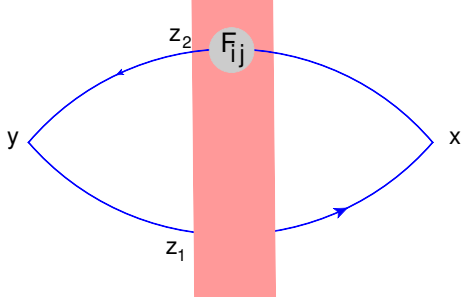

b)

Figure 3. Diagram of the LO impact factor with quark-sub-eikonal correction.

all these contributions together we have

$$
\begin{aligned}
& \langle\mathrm{T}\{\psi(x) \bar{\psi}(y)\}\rangle_{A, \psi, \bar{\psi}}
\end{aligned}
$$

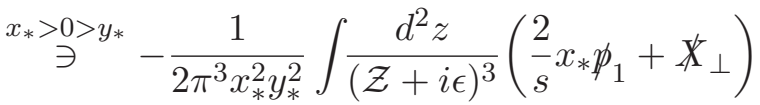

$$
\begin{aligned}
& \times\left\{i \not p_{2} U\left(z_{\perp}\right)+\frac{\mathcal{Z}}{8}\left(\frac{1}{s} \not p_{2} \gamma^{5} \mathcal{F}\left(z_{\perp}\right)+\gamma_{\perp}^{\mu} Q\left(z_{\perp}\right) \gamma_{\mu}^{\perp}\right)\right\}\left(\frac{2}{s} y_{*} \not p_{1}+Y_{\perp}\right)
\end{aligned}
$$

Propagator (3.11) will be used to calculate the impact factor in figure 3.

\section{Operator Product Expansion with sub-eikonal terms}

\subsection{OPE with quark sub-eikonal terms}

Now that we obtained the quark propagator with the necessary sub-eikonal corrections, we are ready to extend the expansion of the T-product of two electromagnetic currents to the polarized DIS case.

The diagram we need to calculate are given in figure 3. As before, we make functional integration over the spinor field

$$
\left\langle\mathrm{T}\left\{j^{\mu}(x) j^{\nu}(y)\right\}\right\rangle_{A, \psi, \bar{\psi}}=\operatorname{tr}\left\{\left\langle x\left|\frac{1}{\not P+i \epsilon}\right| y\right\rangle \gamma^{\nu}\left\langle y\left|\frac{1}{\not P+i \epsilon}\right| x\right\rangle \gamma^{\mu}\right\}
$$

where for each quark propagator in eq. (4.1) we should use eq. (3.11). We will consider operators $\mathcal{F}\left(z_{\perp}\right)$ and $Q\left(z_{\perp}\right)$ separately starting, in this section, with $Q\left(z_{\perp}\right)$.

The product of two quark propagators given in eq. (3.11) will result in four terms. The first term is a product of two eikonal propagators which we considered in section 2 . The second and the third terms are obtained by the product of one eikonal term and one sub-eikonal term; we will consider them in this section. The last term is the product of two sub-eikonal terms, and since it will contribute as a sub-sub-eikonal correction it will be disregarded.

As it is illustrated in figure 3, if we do not want to exceed our precision, we need a sub-eikonal correction to only one of the two interactions between the quark and the 
shock-wave target while keeping eikonal the other one. Diagram 3 a, is

$$
\begin{aligned}
& \left\langle\mathrm{T}\left\{j^{\mu}(x) j^{\nu}(y)\right\}\right\rangle_{\text {Figure } 3 a} \stackrel{x_{*}>0>y_{*}}{\ni} \frac{i}{32 \pi^{6} x_{*}^{4} y_{*}^{4}} \int \frac{d^{2} z_{1} d^{2} z_{2}}{\left[\mathcal{Z}_{1}+i \epsilon\right]^{3}\left[\mathcal{Z}_{2}+i \epsilon\right]^{2}} \\
& \times\left[\operatorname{Tr} \operatorname{tr}\left\{\gamma^{\mu} X_{2} \gamma_{\perp}^{\rho} Q\left(z_{2 \perp}\right) \gamma_{\rho}^{\perp} Y_{2} \gamma^{\nu} Y_{1} \not \not_{2} U_{z_{1}}^{\dagger} X_{1}\right\}\right. \\
& \left.-\left(\operatorname{Tr} \operatorname{tr}\left\{\gamma^{\mu} X_{2} \gamma_{\perp}^{\rho} Q\left(z_{2 \perp}\right) \gamma_{\rho}^{\perp} Y_{2} \gamma^{\nu} Y_{1} \not \not_{2} U_{z_{1}}^{\dagger} X_{1}\right\}\right)^{\dagger}\right]
\end{aligned}
$$

where we used again the short-hand notation $X_{i}^{\mu}=\frac{2}{s} x_{*} p_{1}^{\mu}+X_{i \perp}^{\mu}$ with $X_{i \perp}^{\mu}=x_{\perp}^{\mu}-z_{i \perp}^{\mu}$ and $i=1,2$ and similarly for $Y_{i}$. Moreover, we use tr for trace over spinor indexes while Tr over color indexes (we refer the reader to appendix D for the details of the derivation of eq. (4.2)).

Our task is now the evaluation of the double trace

$$
i \operatorname{Tr} \operatorname{tr}\left\{\gamma^{\mu} X_{2} \gamma_{\perp}^{\rho} Q\left(z_{2 \perp}\right) \gamma_{\rho}^{\perp} Y_{2} \gamma^{\nu} Y_{1} \not \not_{2} U_{z_{1}}^{\dagger} X_{1}\right\}
$$

After some lengthy algebra, and using the rescaling of the spinor field given in eq. (A.3), one can show that the trace over the spinor indexes can be cast in the following form

$$
\begin{aligned}
i \bar{\psi}\left(z_{*}^{\prime}, z_{2 \perp}\right) \gamma_{\rho}^{\perp} \hat{Y}_{2} \gamma^{\nu} \hat{Y}_{1} \not p_{2} \hat{X}_{1} \gamma^{\mu} \hat{X}_{2} \gamma_{\perp}^{\rho} \psi\left(z_{*}, z_{2 \perp}\right) \\
=\frac{8}{s} \bar{\psi}\left(z_{*}^{\prime}, z_{2 \perp}\right) i \not p_{1} \psi\left(z_{*}, z_{2 \perp}\right) I_{1}^{\mu \nu}\left(x_{*}, y_{*} ; z_{1 \perp}, z_{2 \perp}\right) \\
\quad-\frac{8}{s} \bar{\psi}\left(z_{*}^{\prime}, z_{2 \perp}\right) \gamma^{5} \not p_{1} \psi\left(z_{*}, z_{2 \perp}\right) I_{5}^{\mu \nu}\left(x_{*}, y_{*} ; z_{1 \perp}, z_{2 \perp}\right)+O\left(\lambda^{-1}\right)
\end{aligned}
$$

where

$$
I_{1}^{\mu \nu}\left(x, y ; z_{1}, z_{2}\right)=\frac{1}{2} x_{*}^{2} y_{*}^{2} \frac{\partial^{2}}{\partial x_{\mu} \partial y_{\nu}}\left(\mathcal{Z}_{1} \mathcal{Z}_{2}-z_{12 \perp}^{2} \frac{(x-y)^{2}}{x_{*} y_{*}}\right),
$$

with $\mathcal{Z}_{i}$ defined in eq. (2.11), and

$$
I_{5}^{\mu \nu}\left(x, y ; z_{1}, z_{2}\right)=\left(x_{*} \partial_{x}^{\mu}-p_{2}^{\mu}\right)\left(y_{*} \partial_{y}^{\nu}-p_{2}^{\nu}\right)\left[\left(\vec{Y}_{1} \times \vec{Y}_{2}\right) X_{1} \cdot X_{2}-\left(\vec{X}_{1} \times \vec{X}_{2}\right) Y_{1} \cdot Y_{2}\right]
$$

where we used the notation for vector product in two dimensions

$$
\vec{x} \times \vec{y}=\epsilon^{i j} x_{i} y_{j}
$$

The explicit expressions of $I_{1}^{\mu \nu}$ and $I_{5}^{\mu \nu}$ can be found in appendix E.

In result (4.4) we obtained two operators with definite parity. The symmetric tensor $I_{1}^{\mu \nu}$ is proportional to the parity even operator $\bar{\psi}\left(z_{*}^{\prime}, z_{2 \perp}\right) \not p_{1} \psi\left(z_{*}, z_{2 \perp}\right)$, while the operator proportional to the antisymmetric tensor $I_{5}^{\mu \nu}$ is proportional to the parity odd operator $\bar{\psi}\left(z_{*}^{\prime}, z_{2 \perp}\right) \gamma^{5} \not p_{1} \psi\left(z_{*}, z_{2 \perp}\right)$. 
Using result (4.4) in the eq. (4.2), we arrive at

$$
\begin{aligned}
\left\langle\mathrm{T}\left\{j^{\mu}(x) j^{\nu}(y)\right\}\right\rangle_{A, \psi, \bar{\psi}} \stackrel{x_{*}>0>y_{*}}{\ni} \frac{1}{4 \pi^{6} s x_{*}^{4} y_{*}^{4}} \int \frac{d^{2} z_{1} d^{2} z_{2}}{\left[\mathcal{Z}_{1}+i \epsilon\right]^{3}\left[\mathcal{Z}_{2}+i \epsilon\right]^{2}} \\
\times\left[\left(\operatorname{Tr}\left\{\mathcal{Q}_{1 z_{2}} U_{z_{1}}^{\dagger}\right\}+\operatorname{Tr}\left\{\mathcal{Q}_{1 z_{2}}^{\dagger} U_{z_{1}}\right\}\right) I_{1}^{\mu \nu}\left(x, y ; z_{1}, z_{2}\right)\right. \\
\\
\left.-\left(\operatorname{Tr}\left\{\mathcal{Q}_{5 z_{2}} U_{z_{1}}^{\dagger}\right\}+\operatorname{Tr}\left\{\mathcal{Q}_{5 z_{2}}^{\dagger} U_{z_{1}}\right\}\right) I_{5}^{\mu \nu}\left(x, y ; z_{1}, z_{2}\right)\right]
\end{aligned}
$$

where we defined the operators $\mathcal{Q}_{1 z}$ and $\mathcal{Q}_{5 z}$ as

$$
\begin{aligned}
\mathcal{Q}_{1}\left(z_{\perp}\right) \equiv & g^{2} \int_{-\infty}^{+\infty} d z_{*} \int_{-\infty}^{z_{*}} d z_{*}^{\prime}\left[\infty p_{1}, z_{*}\right]_{z} t^{a} \operatorname{tr}\left\{i \not p_{1} \psi\left(z_{*}, z_{\perp}\right)\right. \\
& \left.\times\left[z_{*}, z_{*}^{\prime}\right]_{z}^{a b} \bar{\psi}\left(z_{*}^{\prime}, z_{\perp}\right)\right\} t^{b}\left[z_{*}^{\prime},-\infty p_{1}\right]_{z}, \\
\mathcal{Q}_{5}\left(z_{\perp}\right) \equiv & g^{2} \int_{-\infty}^{+\infty} d z_{*} \int_{-\infty}^{z_{*}} d z_{*}^{\prime}\left[\infty p_{1}, z_{*}\right]_{z} t^{a} \operatorname{tr}\left\{\gamma^{5} \not p_{1} \psi\left(z_{*}, z_{\perp}\right)\right. \\
& \left.\times\left[z_{*}, z_{*}^{\prime}\right]_{z}^{a b} \bar{\psi}\left(z_{*}^{\prime}, z_{\perp}\right)\right\} t^{b}\left[z_{*}^{\prime},-\infty p_{1}\right]_{z} .
\end{aligned}
$$

To put result (4.8) in the same form as eq. (2.16), we define the quark-sub-eikonal impact factors as

$$
\begin{aligned}
& \mathcal{I}_{1}^{\mu \nu}\left(x, y ; z_{1}, z_{2}\right)=\frac{1}{4 \pi^{6} x_{*}^{4} y_{*}^{4}} \frac{I_{1}^{\mu \nu}\left(x, y ; z_{1}, z_{2}\right)}{\left[\mathcal{Z}_{1}+i \epsilon\right]^{3}\left[\mathcal{Z}_{2}+i \epsilon\right]^{2}} \\
& \mathcal{I}_{5}^{\mu \nu}\left(x, y ; z_{1}, z_{2}\right)=-\frac{1}{4 \pi^{6} x_{*}^{4} y_{*}^{4}} \frac{I_{5}^{\mu \nu}\left(x, y ; z_{1}, z_{2}\right)}{\left[\mathcal{Z}_{1}+i \epsilon\right]^{3}\left[\mathcal{Z}_{2}+i \epsilon\right]^{2}}
\end{aligned}
$$

thus the high-energy OPE with quark sub-eikonal contributions is

$$
\begin{aligned}
& T\left\{\overline{\hat{\psi}}(x) \gamma^{\mu} \hat{\psi}(x) \overline{\hat{\psi}}(y) \gamma^{\nu} \hat{\psi}(y)\right\} \\
& \stackrel{x_{*}>0>y_{*}}{\ni} \frac{1}{s} \int d^{2} z_{1} d^{2} z_{2}\left[\left(\operatorname{Tr}\left\{\hat{\mathcal{Q}}_{1 z_{2}} \hat{U}_{z_{1}}^{\dagger}\right\}+\operatorname{Tr}\left\{\hat{\mathcal{Q}}_{1 z_{2}}^{\dagger} \hat{U}_{z_{1}}\right\}\right) \mathcal{I}_{1}^{\mu \nu}\left(x, y ; z_{1}, z_{2}\right)\right. \\
& \left.+\left(\operatorname{Tr}\left\{\hat{\mathcal{Q}}_{5 z_{2}} \hat{U}_{z_{1}}^{\dagger}\right\}+\operatorname{Tr}\left\{\hat{\mathcal{Q}}_{5 z_{2}}^{\dagger} \hat{U}_{z_{1}}\right\}\right) \mathcal{I}_{5}^{\mu \nu}\left(x, y ; z_{1}, z_{2}\right)\right]
\end{aligned}
$$

We notice that the sub-eikonal quark contribution to the quark propagator, eq. (3.9), gave rise to two different impact factors and to two different operators. The impact factor (coefficient function) $\mathcal{I}_{1}$ which is associated to the operator $\operatorname{Tr}\left\{\hat{\mathcal{Q}}_{1 z_{2}} \hat{U}_{z_{1}}^{\dagger}\right\}+\operatorname{Tr}\left\{\hat{\mathcal{Q}}_{1 z_{2}}^{\dagger} \hat{U}_{z_{1}}\right\}$ is a sub-eikonal correction to eikonal unpolarized case eq. (2.16) because it is symmetric under the exchange $\mu \leftrightarrow \nu, x \leftrightarrow y$. Moreover, we notice that there is a relation between the LO impact factor $\mathcal{I}_{L O}^{\mu \nu}$ and the impact factor $\mathcal{I}_{1}^{\mu \nu}$. Indeed, using definitions (2.15) and (4.11) we can write

$$
\mathcal{I}_{1}^{\mu \nu}=\frac{\mathcal{Z}_{2}}{8} \mathcal{I}_{L O}^{\mu \nu}
$$

The impact factor $\mathcal{I}_{5}$ and its associated operator $\operatorname{Tr}\left\{\hat{\mathcal{Q}}_{5 z_{2}} \hat{U}_{z_{1}}^{\dagger}\right\}+\operatorname{Tr}\left\{\hat{\mathcal{Q}}_{5 z_{2}}^{\dagger} \hat{U}_{z_{1}}\right\}$. 
Like the LO impact factor, one can check that the coefficients $\mathcal{I}_{1}^{\mu \nu}$, and $\mathcal{I}_{5}^{\mu \nu}$ in eqs. (4.11) and (4.12) satisfy the electromagnetic gauge invariance

$$
\begin{aligned}
\partial_{\mu}^{x} \frac{1}{\mathcal{Z}_{2}^{2} \mathcal{Z}_{1}^{3} x_{*}^{4} y_{*}^{4}} I_{1}^{\mu \nu}=\frac{1}{x_{*}^{5} y_{*}^{4} \mathcal{Z}_{2}^{3} \mathcal{Z}_{1}^{4}}[ & x_{*} \mathcal{Z}_{1} \mathcal{Z}_{2} \partial_{\mu}^{x} I_{1}^{\mu \nu}-I_{1}^{\mu \nu}\left(4 \mathcal{Z}_{1} \mathcal{Z}_{2} p_{2 \mu}\right. \\
& \left.\left.+3 x_{*} \mathcal{Z}_{2} \partial_{\mu}^{x} \mathcal{Z}_{1}+2 x_{*} \mathcal{Z}_{1} \partial_{\mu}^{x} \mathcal{Z}_{2}\right)\right]=0
\end{aligned}
$$

and

$$
\begin{aligned}
\partial_{\mu}^{x} \frac{1}{\mathcal{Z}_{2}^{2} \mathcal{Z}_{1}^{3} x_{*}^{4} y_{*}^{4}} I_{5}^{\mu \nu}=\frac{1}{x_{*}^{5} y_{*}^{4} \mathcal{Z}_{2}^{3} \mathcal{Z}_{1}^{4}}[ & x_{*} \mathcal{Z}_{1} \mathcal{Z}_{2} \partial_{\mu}^{x} I_{5}^{\mu \nu}-I_{5}^{\mu \nu}\left(4 \mathcal{Z}_{1} \mathcal{Z}_{2} p_{2 \mu}\right. \\
& \left.\left.+3 x_{*} \mathcal{Z}_{2} \partial_{\mu}^{x} \mathcal{Z}_{1}+2 x_{*} \mathcal{Z}_{1} \partial_{\mu}^{x} \mathcal{Z}_{2}\right)\right]=0 .
\end{aligned}
$$

One may also easily check that impact factors (4.11) and (4.12) are Möbius $\operatorname{SL}(2, C)$ invariant.

Before proceeding with the calculation of the evolution equations, we observe that, working out the color algebra we can rewrite operator $Q_{i j}^{\alpha \beta}$ defined in (3.9) as

$$
\begin{aligned}
Q_{i j}^{\alpha \beta}\left(x_{\perp}\right)=-g^{2} \int_{-\infty}^{+\infty} d z_{*} \int_{-\infty}^{z_{*}} d z_{*}^{\prime}[ & \frac{1}{2} U_{x}^{i j} \bar{\psi}^{\alpha}\left(z_{*}^{\prime}, x_{\perp}\right)\left[z_{*}^{\prime}, z_{*}\right]_{x} \psi^{\beta}\left(z_{*}, x_{\perp}\right) \\
& \left.+\frac{1}{2 N_{c}}\left(\left[\infty p_{1}, z_{*}\right]_{x} \psi^{\beta}\left(z_{*}, x_{\perp}\right) \bar{\psi}^{\alpha}\left(z_{*}^{\prime}, x_{\perp}\right)\left[z_{*}^{\prime},-\infty\right]\right)_{i j}\right]
\end{aligned}
$$

From (4.17), we see that in the large $N_{c}$ limit, the $Q_{i j}^{\alpha \beta}$ operator reduces to the product of an infinite Wilson line times the usual light-cone quark operator when multiplied by $\not p_{1}=\sqrt{\frac{s}{2}} \gamma_{+}$(recall that $\gamma_{+}=\gamma^{-}$is what is call the good component) or to the parity odd quark operator when multiplied by $\gamma^{5} \not p_{1}$.

We define the light-ray quark operators as

$$
\begin{aligned}
Q_{1 x} & =-g^{2} \int_{-\infty}^{+\infty} d z_{*} \int_{-\infty}^{z_{*}} d z_{*}^{\prime} \bar{\psi}\left(z_{*}^{\prime}, x_{\perp}\right) i \not p_{1}\left[z_{*}^{\prime}, z_{*}\right]_{x} \psi\left(z_{*}, x_{\perp}\right), \\
Q_{1 x}^{\dagger} & =g^{2} \int_{-\infty}^{+\infty} d z_{*} \int_{-\infty}^{z_{*}} d z_{*}^{\prime} \bar{\psi}\left(z_{*}, x_{\perp}\right) i \not p_{1}\left[z_{*}, z_{*}^{\prime}\right]_{x} \psi\left(z_{*}^{\prime}, x_{\perp}\right), \\
Q_{5 x} & \equiv-g^{2} \int_{-\infty}^{+\infty} d z_{*} \int_{-\infty}^{z_{*}} d z_{*}^{\prime} \bar{\psi}\left(z_{*}^{\prime}, x_{\perp}\right) \gamma^{5} \not p_{1}\left[z_{*}^{\prime}, z_{*}\right]_{x} \psi\left(z_{*}, x_{\perp}\right), \\
Q_{5 x}^{\dagger} & \equiv g^{2} \int_{-\infty}^{+\infty} d z_{*} \int_{-\infty}^{z_{*}} d z_{*}^{\prime} \bar{\psi}\left(z_{*}, x_{\perp}\right) \gamma^{5} \not p_{1}\left[z_{*}, z_{*}^{\prime}\right]_{x} \psi\left(z_{*}^{\prime}, x_{\perp}\right),
\end{aligned}
$$

and

$$
\begin{aligned}
& \tilde{Q}_{1 i j}\left(x_{\perp}\right) \equiv g^{2} \int_{-\infty}^{+\infty} d z_{*} \int_{-\infty}^{z_{*}} d z_{*}^{\prime}\left(\left[\infty p_{1}, z_{*}\right]_{x} \operatorname{tr}\left\{\psi\left(z_{*}, x_{\perp}\right) \bar{\psi}\left(z_{*}^{\prime}, x_{\perp}\right) i \not p_{1}\right\}\left[z_{*}^{\prime},-\infty p_{1}\right]\right)_{i j}, \\
& \tilde{Q}_{1 i j}^{\dagger}\left(x_{\perp}\right) \equiv-g^{2} \int_{-\infty}^{+\infty} d z_{*} \int_{-\infty}^{z_{*}} d z_{*}^{\prime}\left(\left[-\infty p_{1}, z_{*}^{\prime}\right]_{x} \operatorname{tr}\left\{\psi\left(z_{*}^{\prime}, x_{\perp}\right) \bar{\psi}\left(z_{*}, x_{\perp}\right) i \not p_{1}\right\}\left[z_{*}, \infty p_{1}\right]\right)_{i j}, \\
& \tilde{Q}_{5 i j}\left(x_{\perp}\right) \equiv g^{2} \int_{-\infty}^{+\infty} d z_{*} \int_{-\infty}^{z_{*}} d z_{*}^{\prime}\left(\left[\infty p_{1}, z_{*}\right]_{x} \operatorname{tr}\left\{\psi\left(z_{*}, x_{\perp}\right) \bar{\psi}\left(z_{*}^{\prime}, x_{\perp}\right) \gamma^{5} \not p_{1}\right\}\left[z_{*}^{\prime},-\infty p_{1}\right]\right)_{i j} \\
& \tilde{Q}_{5 i j}^{\dagger}\left(x_{\perp}\right) \equiv g^{2} \int_{-\infty}^{+\infty} d z_{*} \int_{-\infty}^{z_{*}} d z_{*}^{\prime}\left(\left[-\infty p_{1}, z_{*}^{\prime}\right]_{x} \operatorname{tr}\left\{\psi\left(z_{*}^{\prime}, x_{\perp}\right) \bar{\psi}\left(z_{*}, x_{\perp}\right) \gamma^{5} \not p_{1}\right\}\left[z_{*}, \infty p_{1}\right]\right)_{i j}
\end{aligned}
$$




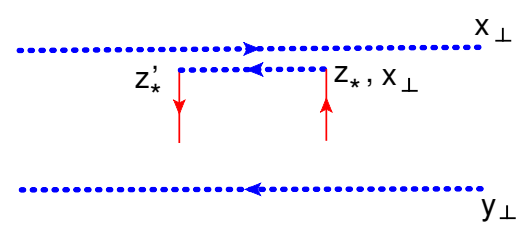

a)

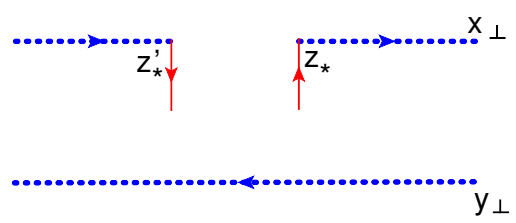

b)

Figure 4. Figure a) represents the operators $\operatorname{Tr}\left\{U_{x} U_{y}^{\dagger}\right\} Q_{1 x}$, and $\operatorname{Tr}\left\{U_{x} U_{y}^{\dagger}\right\} Q_{5 x}$, while figure b) represents operators $\operatorname{Tr}\left\{\tilde{Q}_{1 x} U_{y}^{\dagger}\right\}$, and $\operatorname{Tr}\left\{\tilde{Q}_{5 x} U_{y}^{\dagger}\right\}$.

where $i, j$ are color indexes in the fundamental representation. So, we can rewrite the operator $\operatorname{Tr}\left\{\hat{\mathcal{Q}}_{1 x} \hat{U}_{y}^{\dagger}\right\}$, and $\operatorname{Tr}\left\{\hat{\mathcal{Q}}_{5 x} \hat{U}_{y}^{\dagger}\right\}$, and their adjoint conjugated as

$$
\begin{aligned}
\operatorname{Tr}\left\{\hat{\mathcal{Q}}_{1 x} \hat{U}_{y}^{\dagger}\right\} & =\frac{1}{2} \operatorname{Tr}\left\{\hat{U}_{y}^{\dagger} \hat{U}_{x}\right\} \hat{Q}_{1 x}-\frac{1}{2 N_{c}} \operatorname{Tr}\left\{\hat{U}_{y}^{\dagger} \hat{\tilde{Q}}_{1 x}\right\} \\
\operatorname{Tr}\left\{\hat{\mathcal{Q}}_{1 x}^{\dagger} \hat{U}_{y}\right\} & =\frac{1}{2} \operatorname{Tr}\left\{\hat{U}_{y} \hat{U}_{x}^{\dagger}\right\} \hat{Q}_{1 x}^{\dagger}-\frac{1}{2 N_{c}} \operatorname{Tr}\left\{\hat{U}_{y} \hat{\tilde{Q}}_{1 x}^{\dagger}\right\} \\
\operatorname{Tr}\left\{\hat{\mathcal{Q}}_{5 x} \hat{U}_{y}^{\dagger}\right\} & =\frac{1}{2} \operatorname{Tr}\left\{\hat{U}_{y}^{\dagger} \hat{U}_{x}\right\} \hat{Q}_{5 x}-\frac{1}{2 N_{c}} \operatorname{Tr}\left\{\hat{U}_{y}^{\dagger} \hat{\tilde{Q}}_{5 x}\right\} \\
\operatorname{Tr}\left\{\hat{\mathcal{Q}}_{5 x}^{\dagger} \hat{U}_{y}\right\} & =\frac{1}{2} \operatorname{Tr}\left\{\hat{U}_{y} \hat{U}_{x}^{\dagger}\right\} \hat{Q}_{5 x}^{\dagger}-\frac{1}{2 N_{c}} \operatorname{Tr}\left\{\hat{U}_{y} \hat{\tilde{Q}}_{5 x}^{\dagger}\right\}
\end{aligned}
$$

Operators (4.26)-(4.29) are the new operators of which we will calculate the evolution equations and in provide the parametrization through new distribution functions. They are represented in figure 4.

The OPE (4.13) in terms of operators (4.26)-(4.29) is

$$
\begin{aligned}
& T\left\{\overline{\hat{\psi}}(x) \gamma^{\mu} \hat{\psi}(x) \overline{\hat{\psi}}(y) \gamma^{\nu} \hat{\psi}(y)\right\} \\
& \stackrel{x_{*}>0>y_{*}}{\ni} \frac{1}{2 s} \int d^{2} z_{1} d^{2} z_{2}\left[\mathcal { I } _ { 1 } ^ { \mu \nu } ( x , y ; z _ { 1 } , z _ { 2 } ) \left(\operatorname{Tr}\left\{\hat{U}_{z_{1}}^{\dagger} \hat{U}_{z_{2}}\right\} \hat{Q}_{1 z_{2}}+\operatorname{Tr}\left\{\hat{U}_{z_{1}} \hat{U}_{z_{2}}^{\dagger}\right\} \hat{Q}_{1 z_{2}}^{\dagger}\right.\right. \\
& \left.-\frac{1}{N_{c}} \operatorname{Tr}\left\{\hat{U}_{z_{1}}^{\dagger} \hat{\tilde{Q}}_{1 z_{2}}\right\}-\frac{1}{N_{c}} \operatorname{Tr}\left\{\hat{U}_{z_{1}} \hat{\tilde{Q}}_{1 z_{2}}^{\dagger}\right\}\right) \\
& +\mathcal{I}_{5}^{\mu \nu}\left(x, y ; z_{1}, z_{2}\right)\left(\operatorname{Tr}\left\{\hat{U}_{z_{1}}^{\dagger} \hat{U}_{z_{2}}\right\} \hat{Q}_{5 z_{2}}+\operatorname{Tr}\left\{\hat{U}_{z_{1}} \hat{U}_{z_{2}}^{\dagger}\right\} \hat{Q}_{5 z_{2}}^{\dagger}\right. \\
& \left.\left.-\frac{1}{N_{c}} \operatorname{Tr}\left\{\hat{U}_{z_{1}}^{\dagger} \hat{\tilde{Q}}_{5 z_{2}}\right\}-\frac{1}{N_{c}} \operatorname{Tr}\left\{\hat{U}_{z_{1}} \hat{\tilde{Q}}_{5 z_{2}}^{\dagger}\right\}\right)\right]
\end{aligned}
$$

\subsection{OPE with gluon sub-eikonal terms}

The contribution to the high-energy OPE of the T-product of two electromagnetic currents due to sub-eikonal terms in the background of gluon fields, given in eq. (3.4), is obtained 
again by performing the spinor contractions (see figure $3 \mathrm{~b}$ )

$$
\begin{aligned}
\left\langle\bar{\psi}(x) \gamma^{\mu} \psi(x) \bar{\psi}(y) \gamma^{\nu} \psi(y)\right\rangle_{A} & \operatorname{tr}\left\{\left\langle x\left|\frac{1}{\not P+i \epsilon}\right| y\right\rangle \gamma^{\nu}\left\langle y\left|\frac{1}{\not P+i \epsilon}\right| x\right\rangle \gamma^{\mu}\right\} \\
x_{*}>0>y_{*} & \frac{g}{64 \pi^{6}} \int d^{2} z_{1} d^{2} z_{2} \frac{\left[\mathcal{Z}_{2}+i \epsilon\right]}{\left[\mathcal{Z}_{1}+i \epsilon\right]^{3}} \operatorname{tr}\left\{X_{1} \not p_{2} Y_{1} \gamma^{\nu} Y_{2} \not p_{2} \sigma_{\perp}^{\rho \sigma} X_{2} \gamma^{\mu}\right\} \\
\times & {\left[\int_{-\infty}^{+\infty} d \omega_{*} \operatorname{Tr}\left\{\left[\infty p_{1}, \omega_{*}\right]_{z_{2}} F_{\rho \sigma}^{\perp}\left[\omega_{*},-\infty p_{1}\right]_{z_{2}} U_{z_{1}}^{\dagger}\right\}\right.} \\
& \left.\quad-\int_{-\infty}^{+\infty} d \omega_{*} \operatorname{Tr}\left\{U_{z_{1}}\left[-\infty p_{1}, \omega_{*}\right]_{z_{2}} F_{\rho \sigma}^{\perp}\left[\omega_{*}, \infty p_{1}\right]_{z_{2}}\right\}\right] .
\end{aligned}
$$

After a lengthy algebra, the calculation of the gamma matrices in (4.31) gives

$$
\begin{aligned}
\operatorname{tr}\left\{X_{1} \not p_{2} Y_{1} \gamma^{\nu} Y_{2} \not p_{2} \sigma_{\perp}^{\alpha \beta} X_{2} \gamma^{\mu}\right\} \\
\quad=8 i \epsilon^{\alpha \beta}\left(x_{*} \partial_{x}^{\mu}-p_{2}^{\mu}\right)\left(y_{*} \partial_{y}^{\nu}-p_{2}^{\nu}\right)\left[\left(\vec{X}_{1} \times \vec{X}_{2}\right) Y_{1} \cdot Y_{2}-\left(\vec{Y}_{1} \times \vec{Y}_{2}\right) X_{1} \cdot X_{2}\right] \\
\quad \equiv 8 i \epsilon^{\alpha \beta} I_{\mathcal{F}}^{\mu \nu} .
\end{aligned}
$$

So, it turns out that $I_{\mathcal{F}}^{\mu \nu}=-I_{5}^{\mu \nu}$. This should not be surprising because the two operators, $\mathcal{Q}_{5 x}$ and $\mathcal{F}_{x}$ are both parity odd.

Using the operator $\mathcal{F}_{z}$ defined in eq. (3.6), eq. (4.31) becomes

$$
\begin{aligned}
& \left\langle\bar{\psi}(x) \gamma^{\mu} \psi(x) \bar{\psi}(y) \gamma^{\nu} \psi(y)\right\rangle_{A} \\
& \quad=\operatorname{tr}\left\{\left\langle x\left|\frac{1}{\not P+i \epsilon}\right| y\right\rangle \gamma^{\nu}\left\langle y\left|\frac{1}{\not P+i \epsilon}\right| x\right\rangle \gamma^{\mu}\right\} \\
& \stackrel{x_{*}>0>y_{*}}{\quad} \frac{1}{4 \pi^{6} s x_{*}^{4} y_{*}^{4}} \int d^{2} z_{1} d^{2} z_{2} \frac{\left[\mathcal{Z}_{2}+i \epsilon\right]^{-2}}{\left[\mathcal{Z}_{1}+i \epsilon\right]^{3}} I_{\mathcal{F}}^{\mu \nu}\left(z_{1}, z_{1} ; x, y\right)\left[\operatorname{Tr}\left\{U_{z_{1}}^{\dagger} \mathcal{F}_{z_{2}}\right\}+\operatorname{Tr}\left\{U_{z_{1}} \mathcal{F}_{z_{2}}^{\dagger}\right\}\right] .
\end{aligned}
$$

and using the definition of $\mathcal{I}_{5}^{\mu \nu},(4.33)$ can be written as

$$
\begin{aligned}
\mathrm{T}\left\{\hat{\bar{\psi}}(x) \gamma^{\mu} \psi(x) \overline{\hat{\psi}}(y) \gamma^{\nu} \hat{\psi}(y)\right\} \\
\stackrel{x_{*}>0>y_{*}}{\ni} \frac{1}{s} \int d^{2} z_{1} d^{2} z_{2} \mathcal{I}_{5}^{\mu \nu}\left(z_{1 \perp}, z_{2 \perp} ; x, y\right)\left[\operatorname{Tr}\left\{\hat{U}_{z_{1}}^{\dagger} \hat{\mathcal{F}}_{z_{2}}\right\}+\operatorname{Tr}\left\{\hat{U}_{z_{1}} \hat{\mathcal{F}}_{z_{2}}^{\dagger}\right\}\right]
\end{aligned}
$$

therefore, the coefficient $\mathcal{I}_{5}^{\mu \nu}$ is also the impact-factor associated to the operator $\operatorname{Tr}\left\{\hat{U}_{z_{1}}^{\dagger} \hat{\mathcal{F}}\left(z_{2 \perp}\right)\right\}+\operatorname{Tr}\left\{\hat{U}_{z_{1}} \hat{\mathcal{F}}^{\dagger}\left(z_{2 \perp}\right)\right\}$.

\subsection{OPE with sub-eikonal corrections: flavor singlet}

We can now add together the contributions of the quark, eq. (4.13), and gluon, eq. (4.34), sub-eikonal corrections. We have

$$
\begin{aligned}
& \mathrm{T}\left\{\overline{\hat{\psi}}(x) \gamma^{\mu} \psi(x) \overline{\hat{\psi}}(y) \gamma^{\nu} \hat{\psi}(y)\right\} \\
& =\int d z_{1} d z_{2} \mathcal{I}_{L O}^{\mu \nu}\left(z_{1 \perp}, z_{2 \perp} ; x, y\right)\left[\operatorname{Tr}\left\{\hat{U}_{z_{1}} \hat{U}_{z_{2}}^{\dagger}\right\}+\frac{\mathcal{Z}_{2}}{8 s}\left(\operatorname{Tr}\left\{\hat{U}_{z_{1}}^{\dagger} \hat{\mathcal{Q}}_{1 z_{2}}\right\}+\operatorname{Tr}\left\{\hat{U}_{z_{1}} \hat{\mathcal{Q}}_{1 z_{2}}^{\dagger}\right\}\right)\right] \\
& +\frac{1}{s} \int d^{2} z_{1} d^{2} z_{2} \mathcal{I}_{5}^{\mu \nu}\left(z_{1 \perp}, z_{2 \perp} ; x, y\right)\left[\operatorname{Tr}\left\{\left(\hat{\mathcal{Q}}_{5 z_{2}}+\hat{\mathcal{F}}_{z_{2}}\right) \hat{U}_{z_{1}}^{\dagger}\right\}+\operatorname{Tr}\left\{\left(\hat{\mathcal{Q}}_{5 z_{2}}^{\dagger}+\hat{\mathcal{F}}_{z_{2}}^{\dagger}\right) \hat{U}_{z_{1}}\right\}\right] \\
& +\mathcal{O}\left(\alpha_{s}\right)+\mathcal{O}\left(\lambda^{-2}\right)
\end{aligned}
$$


The same expansion can be written in terms of the operators $\hat{Q}_{1}\left(x_{\perp}\right)$ and $\hat{Q}_{5}\left(x_{\perp}\right), \hat{\tilde{Q}}_{1}\left(x_{\perp}\right)$ and $\hat{\tilde{Q}}_{5}\left(x_{\perp}\right)$ and their adjoint conjugated as

$$
\begin{aligned}
& \operatorname{T}\left\{\overline{\hat{\psi}}(x) \gamma^{\mu} \psi(x) \overline{\hat{\psi}}(y) \gamma^{\nu} \hat{\psi}(y)\right\} \\
= & \int d z_{1} d z_{2} \mathcal{I}_{L O}^{\mu \nu}\left(z_{1 \perp}, z_{2 \perp} ; x, y\right)\left[\operatorname{Tr}\left\{\hat{U}_{z_{1}}^{\dagger} \hat{U}_{z_{2}}^{\dagger}\right\}\right. \\
& \left.+\frac{\mathcal{Z}_{2}}{16 s}\left(\operatorname{Tr}\left\{\hat{U}_{z_{1}}^{\dagger} \hat{U}_{z_{2}}\right\} \hat{Q}_{1 z_{2}}+\operatorname{Tr}\left\{\hat{U}_{z_{1}} \hat{U}_{z_{2}}^{\dagger}\right\} \hat{Q}_{1 z_{2}}^{\dagger}-\frac{1}{N_{c}} \operatorname{Tr}\left\{\hat{U}_{z_{1}}^{\dagger} \hat{\tilde{Q}}_{1 z_{2}}\right\}-\frac{1}{N_{c}} \operatorname{Tr}\left\{\hat{U}_{z_{1}} \hat{\tilde{Q}}_{1 z_{2}}^{\dagger}\right\}\right)\right] \\
& +\frac{1}{s} \int d^{2} z_{1} d^{2} z_{2} \mathcal{I}_{5}^{\mu \nu}\left(z_{1 \perp}, z_{2 \perp} ; x, y\right)\left[\operatorname{Tr}\left\{\hat{U}_{z_{1}}^{\dagger} \hat{U}_{z_{2}}\right\} \hat{Q}_{5 z_{2}}+\operatorname{Tr}\left\{\hat{U}_{z_{1}} \hat{U}_{z_{2}}^{\dagger}\right\} \hat{Q}_{5 z_{2}}^{\dagger}\right. \\
& \left.-\frac{1}{N_{c}} \operatorname{Tr}\left\{\hat{U}_{z_{1}}^{\dagger}\left(\hat{\tilde{Q}}_{5 z_{2}}-2 N_{c} \hat{\mathcal{F}}_{z_{2}}\right)\right\}-\frac{1}{N_{c}} \operatorname{Tr}\left\{\hat{U}_{z_{1}}\left(\hat{\tilde{Q}}_{5 z_{2}}^{\dagger}-2 N_{c} \hat{\mathcal{F}}_{z_{2}}^{\dagger}\right)\right\}\right] \\
& +\mathcal{O}\left(\alpha_{s}\right)+\mathcal{O}\left(\lambda^{-2}\right)
\end{aligned}
$$

Equations (4.36) and (4.35) are equivalent, and are the high-energy OPE with the subeikonal corrections that come in with a $\frac{1}{s}$ factor. In principle, if we consider the full quark propagator in the background of gluon field, (3.1), there might be other sub-eikonal contributions to the unpolarized and polarized high-energy OPE (see appendix C for more details). In other words, if we use the full quark propagator (3.1), from diagram in figure $3 \mathrm{~b}$ we will get other terms besides the one we obtained using only the term proportional to $\epsilon^{i j} F_{i j}$. However the new terms will not contribute to $g_{1}$ structure function.

Considering that both $\hat{\mathcal{Q}}_{5 x}$ and $\hat{\mathcal{F}}_{x}$ are parity odd operators and that their impact factors results, which came from two independent and different calculations, are equal, we may consider this as an indirect proof of the validity of the entire result.

At small-x, the unpolarized quark structure function is known to be energy suppressed with respect to the gluon structure function. Indeed, this can be observed from our OPE result (4.36), where the sub-eikonal impact factors $\mathcal{I}_{1}^{\mu \nu}$ and $\mathcal{I}_{5}^{\mu \nu}$ are proportional to $\frac{1}{s}$.

In the large $N_{c}$ approximation we have

$$
\begin{aligned}
\mathrm{T}\{\overline{\hat{\psi}}(x) & \left.\gamma^{\mu} \psi(x) \overline{\hat{\psi}}(y) \gamma^{\nu} \hat{\psi}(y)\right\} \\
= & \int d z_{1} d z_{2}\left\{\mathcal{I}_{L O}^{\mu \nu}\left(z_{1 \perp}, z_{2 \perp} ; x, y\right) \operatorname{Tr}\left\{\hat{U}_{z_{1}} \hat{U}_{z_{2}}^{\dagger}\right\}\right. \\
& +\frac{1}{2 s} \mathcal{I}_{1}^{\mu \nu}\left(z_{1 \perp}, z_{2 \perp} ; x, y\right)\left(\operatorname{Tr}\left\{\hat{U}_{z_{1}}^{\dagger} \hat{U}_{z_{2}}\right\} \hat{Q}_{1 z_{2}}+\operatorname{Tr}\left\{\hat{U}_{z_{1}} \hat{U}_{z_{2}}^{\dagger}\right\} \hat{Q}_{1 z_{2}}^{\dagger}\right) \\
& +\frac{1}{2 s} \mathcal{I}_{5}^{\mu \nu}\left(z_{1 \perp}, z_{2 \perp} ; x, y\right)\left(\operatorname{Tr}\left\{\hat{U}_{z_{1}}^{\dagger} \hat{U}_{z_{2}}\right\} \hat{Q}_{5 z_{2}}+\operatorname{Tr}\left\{\hat{U}_{z_{1}} \hat{U}_{z_{2}}^{\dagger}\right\} \hat{Q}_{5 z_{2}}^{\dagger}\right. \\
& \left.\left.+2 \operatorname{Tr}\left\{U_{z_{1}}^{\dagger} \mathcal{F}_{z_{2}}\right\}+2 \operatorname{Tr}\left\{U_{z_{1}} \mathcal{F}_{z_{2}}^{\dagger}\right\}\right)\right\}+\mathcal{O}\left(\alpha_{s}\right)+\mathcal{O}\left(\lambda^{-2}\right)+\mathcal{O}\left(1 / N_{c}\right),
\end{aligned}
$$

(recall that $\left.\mathcal{I}_{1}^{\mu \nu}=\frac{\mathcal{Z}_{2}}{8} \mathcal{I}_{L O}^{\mu \nu}\right)$.

\subsection{OPE with sub-eikonal contributions: flavor non-singlet}

In the flavor non-singlet case, the high-energy OPE will be the same as in (4.36) with the exception that the operator $\hat{\mathcal{F}}\left(x_{\perp}\right)$ will be absent because it does not allow exchange of 
flavor with the target. So we have

$$
\begin{aligned}
& \operatorname{T}\left\{\overline{\hat{\psi}}(x) \gamma^{\mu} \psi(x) \overline{\hat{\psi}}(y) \gamma^{\nu} \hat{\psi}(y)\right\} \\
& =\int d z_{1} d z_{2} \mathcal{I}_{L O}^{\mu \nu}\left(z_{1 \perp}, z_{2 \perp} ; x, y\right)\left[\operatorname{Tr}\left\{\hat{U}_{z_{1}} \hat{U}_{z_{2}}^{\dagger}\right\}+\frac{\mathcal{Z}_{2}}{8 s}\left(\operatorname{Tr}\left\{U_{z_{1}}^{\dagger} \mathcal{Q}_{1 z_{2}}\right\}+\operatorname{Tr}\left\{U_{z_{1}} \mathcal{Q}_{1 z_{2}}^{\dagger}\right\}\right)\right] \\
& \quad+\frac{1}{s} \int d^{2} z_{1} d^{2} z_{2} \mathcal{I}_{5}^{\mu \nu}\left(z_{1 \perp}, z_{2 \perp} ; x, y\right)\left[\operatorname{Tr}\left\{\hat{\mathcal{Q}}_{5 z_{2}} \hat{U}_{z_{1}}^{\dagger}\right\}+\operatorname{Tr}\left\{\hat{\mathcal{Q}}_{5 z_{2}}^{\dagger} \hat{U}_{z_{1}}\right\}\right] \\
& \quad+\mathcal{O}\left(\alpha_{s}\right)+\mathcal{O}\left(\lambda^{-2}\right)
\end{aligned}
$$

As for the singlet case, the same expansion can be written in terms of the operators $\hat{Q}_{1}\left(x_{\perp}\right)$ and $\hat{Q}_{5}\left(x_{\perp}\right), \hat{\tilde{Q}}_{1}\left(x_{\perp}\right)$ and $\hat{\tilde{Q}}_{5}\left(x_{\perp}\right)$ and their adjoint conjugated as

$$
\begin{aligned}
& \operatorname{T}\left\{\overline{\hat{\psi}}(x) \gamma^{\mu} \psi(x) \overline{\hat{\psi}}(y) \gamma^{\nu} \hat{\psi}(y)\right\} \\
& =\int d z_{1} d z_{2}\left\{\mathcal{I}_{L O}^{\mu \nu}\left(z_{1 \perp}, z_{2 \perp} ; x, y\right)+\frac{\mathcal{Z}_{2}}{16 s} \mathcal{I}_{L O}^{\mu \nu}\left(z_{1 \perp}, z_{2 \perp} ; x, y\right)\left(\operatorname{Tr}\left\{\hat{U}_{z_{1}}^{\dagger} \hat{U}_{z_{2}}\right\} \hat{Q}_{1 z_{2}}\right.\right. \\
& \left.+\operatorname{Tr}\left\{\hat{U}_{z_{1}} \hat{U}_{z_{2}}^{\dagger}\right\} \hat{Q}_{1 z_{2}}^{\dagger}-\frac{1}{N_{c}} \operatorname{Tr}\left\{\hat{U}_{z_{1}}^{\dagger} \hat{\tilde{Q}}_{1 z_{2}}\right\}-\frac{1}{N_{c}} \operatorname{Tr}\left\{\hat{U}_{z_{1}} \hat{\tilde{Q}}_{1 z_{2}}^{\dagger}\right\}\right) \\
& \quad+\frac{1}{s} \mathcal{I}_{5}^{\mu \nu}\left(z_{1 \perp}, z_{2 \perp} ; x, y\right)\left(\operatorname{Tr}\left\{\hat{U}_{z_{1}}^{\dagger} \hat{U}_{z_{2}}\right\} \hat{Q}_{5 z_{2}}+\operatorname{Tr}\left\{\hat{U}_{z_{1}} \hat{U}_{z_{2}}^{\dagger}\right\} \hat{Q}_{5 z_{2}}^{\dagger}\right. \\
& \left.\left.\quad-\frac{1}{N_{c}} \operatorname{Tr}\left\{\hat{U}_{z_{1}}^{\dagger} \hat{\tilde{Q}}_{5 z_{2}}\right\}-\frac{1}{N_{c}} \operatorname{Tr}\left\{\hat{U}_{z_{1}} \hat{\tilde{Q}}_{5 z_{2}}^{\dagger}\right\}\right)+\mathcal{O}\left(\alpha_{s}\right)+\mathcal{O}\left(\lambda^{-2}\right)\right\}
\end{aligned}
$$

In the large $N_{c}$ approximation we can simplify to

$$
\begin{aligned}
& \operatorname{T}\left\{\overline{\hat{\psi}}(x) \gamma^{\mu} \psi(x) \overline{\hat{\psi}}(y) \gamma^{\nu} \hat{\psi}(y)\right\} \\
&=\int d z_{1} d z_{2}\{\left\{\mathcal{I}_{L O}^{\mu \nu}\left(z_{1 \perp}, z_{2 \perp} ; x, y\right)\right. \\
&+\frac{\mathcal{Z}_{2}}{16 s} \mathcal{I}_{L O}^{\mu \nu}\left(z_{1 \perp}, z_{2 \perp} ; x, y\right)\left(\operatorname{Tr}\left\{\hat{U}_{z_{1}}^{\dagger} \hat{U}_{z_{2}}\right\} \hat{Q}_{1 z_{2}}+\operatorname{Tr}\left\{\hat{U}_{z_{1}} \hat{U}_{z_{2}}^{\dagger}\right\} \hat{Q}_{1 z_{2}}^{\dagger}\right) \\
&+\frac{1}{s} \mathcal{I}_{5}^{\mu \nu}\left(z_{1 \perp}, z_{2 \perp} ; x, y\right)\left(\operatorname{Tr}\left\{\hat{U}_{z_{1}}^{\dagger} \hat{U}_{z_{2}}\right\} \hat{Q}_{5 z_{2}}+\operatorname{Tr}\left\{\hat{U}_{z_{1}} \hat{U}_{z_{2}}^{\dagger}\right\} \hat{Q}_{5 z_{2}}^{\dagger}\right) \\
&\left.+\mathcal{O}\left(\alpha_{s}\right)+\mathcal{O}\left(\lambda^{-2}\right)+\mathcal{O}\left(1 / N_{c}\right)\right\}
\end{aligned}
$$

We will find the evolution of these operators in the next sections.

\section{Parametrization of the forward matrix elements}

In this section we give an account of the operators that we have found and provide their parametrization through new distribution functions (all of dimensions $\left[M^{-2}\right]$ ).

We denote by $S_{L}^{\mu}$ the longitudinal spin of the hadronic target. In the DIS kinematics we have $M S_{L}^{\mu} \simeq \lambda P^{\mu}$ so, we may write $S^{\mu} \simeq \frac{\lambda}{M} P^{\mu}+S_{\perp}^{\mu}$ with helicity $\lambda$.

To consider forward matrix elements, we need to define the reduced matrix elements. Consider an operator $\hat{O}\left(x_{\perp}, y_{\perp}\right)$, function of two transverse distances, which can be one of 
the dipole type of operators we are going to consider in this section. Then, we define the reduced matrix elements as

$$
\left\langle P, S\left|\hat{O}\left(k_{\perp}\right)\right| P^{\prime}, S\right\rangle=2 \pi \frac{s}{2} \delta\left(\left(P^{\prime}-P\right) \cdot p_{1}\right)\left\langle\left\langle P, S\left|\hat{O}\left(k_{\perp}\right)\right| P, S\right\rangle\right\rangle
$$

with $P^{\prime \mu}=P^{\mu}+\beta p_{2}^{\mu}$ and

$$
\int d^{2} \Delta e^{-i(k, \Delta)_{\perp}}\left\langle\left\langle P, S\left|\hat{O}\left(x_{\perp}, y_{\perp}\right)\right| P, S\right\rangle\right\rangle=\left\langle\left\langle P, S\left|\hat{O}\left(k_{\perp}\right)\right| P, S\right\rangle\right\rangle
$$

where we defined $\Delta_{\perp}^{\mu} \equiv(x-y)_{\perp}^{\mu}$. The delta function $\delta\left(\left(P^{\prime}-P\right) \cdot p_{1}\right)$ takes into account that forward matrix elements of dipole type operators contain unrestricted integration along $p_{1}$.

Let us start considering the relevant matrix elements. Consider matrix element with operator $\hat{Q}_{1}\left(x_{\perp}\right)$

$$
\begin{aligned}
\int d^{2} \Delta & \left.e^{i(\Delta, k)}\left\langle\langle P, S|\left[Q_{1}\left(x_{\perp}\right) \operatorname{Tr}\left\{U_{x} U_{y}^{\dagger}\right\}+\text { a.c. }\right] \mid P, S\right\rangle\right\rangle \\
= & -i g^{2} \int d^{2} \Delta e^{i(\Delta, k)} \int_{-\infty}^{+\infty} d z_{*} \int_{-\infty}^{z_{*}} d z_{*}^{\prime} \\
& \left.\times\left\langle\langle P, S|\left[\bar{\psi}\left(z_{*}^{\prime}, x_{\perp}\right) \not p_{1}\left[z_{*}^{\prime}, z_{*}\right]_{x} \psi\left(z_{*}, x_{\perp}\right) \operatorname{Tr}\left\{U_{x} U_{y}^{\dagger}\right\}+\text { a.c }\right] \mid P, S\right\rangle\right\rangle \\
= & \frac{s}{2}\left(q_{1}\left(k_{\perp}^{2}, x\right)+\frac{\vec{S} \times \vec{k}}{M} q_{1 T}\left(k_{\perp}^{2}, x\right)\right) .
\end{aligned}
$$

with $\epsilon^{i j} S_{i} k_{j}=\vec{S} \times \vec{k}$. (recall $z_{*}, z_{*}^{\prime}$ are dimensionless) and a.c stands for adjoint conjugated.

Matrix element with operator $\hat{\tilde{Q}}_{1}\left(x_{\perp}\right)$

$$
\begin{aligned}
\int d^{2} \Delta & \left.e^{i(\Delta, k)}\left\langle\langle P, S|\left[\operatorname{Tr}\left\{\tilde{Q}_{1}\left(x_{\perp}\right) U_{y}^{\dagger}\right\}+\text { a.c. }\right] \mid P, S\right\rangle\right\rangle \\
= & i g^{2} \int d^{2} \Delta e^{i(\Delta, k)} \int_{-\infty}^{+\infty} d z_{*} \int_{-\infty}^{z_{*}} d z_{*}^{\prime} \\
& \left.\times\left\langle\langle P, S|\left[\operatorname{Tr}\left\{\left[\infty p_{1}, z_{*}\right]_{x} \operatorname{tr}\left\{\psi\left(z_{*}, x_{\perp}\right) \bar{\psi}\left(z_{*}^{\prime}, x_{\perp}\right) \not p_{1}\right\}\left[z_{*}^{\prime},-\infty p_{1}\right] U_{y}^{\dagger}\right\}+\text { a.c. }\right] \mid P, S\right\rangle\right\rangle \\
= & \frac{s}{2}\left(\tilde{q}_{1}\left(k_{\perp}^{2}, x\right)+\frac{\vec{S} \times \vec{k}}{M} \tilde{q}_{1 T}\left(k_{\perp}^{2}, x\right)\right)
\end{aligned}
$$

Matrix element with operator $\hat{Q}_{5}\left(x_{\perp}\right)$

$$
\begin{aligned}
\int d^{2} \Delta & \left.e^{i(\Delta, k)}\left\langle\langle P, S|\left[Q_{5}\left(x_{\perp}\right) \operatorname{Tr}\left\{U_{x} U_{y}^{\dagger}\right\}+\text { a.c }\right] \mid P, S\right\rangle\right\rangle \\
= & -g^{2} \int d^{2} \Delta e^{i(\Delta, k)} \int_{-\infty}^{+\infty} d z_{*} \int_{-\infty}^{z_{*}} d z_{*}^{\prime} \\
& \left.\times\left\langle\langle P, S|\left[\bar{\psi}\left(z_{*}^{\prime}, x_{\perp}\right) \gamma^{5} \not p_{1}\left[z_{*}^{\prime}, z_{*}\right]_{x} \psi\left(z_{*}, x_{\perp}\right) \operatorname{Tr}\left\{U_{x} U_{y}^{\dagger}\right\}+\text { a.c }\right] \mid P, S\right\rangle\right\rangle \\
= & \frac{s}{2}\left(\lambda q_{5 L}\left(k_{\perp}^{2}, x\right)-\frac{(S, k)_{\perp}}{M} q_{5 T}\left(k_{\perp}^{2}, x\right)\right) .
\end{aligned}
$$


Matrix element with operator $\hat{\tilde{Q}}_{5}\left(x_{\perp}\right)$

$$
\begin{aligned}
\int d^{2} \Delta & \left.e^{i(\Delta, k)}\left\langle\langle P, S|\left[\operatorname{Tr}\left\{\tilde{Q}_{5}\left(x_{\perp}\right) U_{y}^{\dagger}\right\}+\text { a.c }\right] \mid P, S\right\rangle\right\rangle \\
= & g^{2} \int d^{2} \Delta e^{i(\Delta, k)} \int_{-\infty}^{+\infty} d z_{*} \int_{-\infty}^{z_{*}} d z_{*}^{\prime} \\
& \left.\times\left\langle\langle P, S|\left[\operatorname{Tr}\left\{\left[\infty p_{1}, z_{*}\right]_{x} \operatorname{tr}\left\{\psi\left(z_{*}, x_{\perp}\right) \bar{\psi}\left(z_{*}^{\prime}, x_{\perp}\right) \gamma^{5} \not p_{1}\right\}\left[z_{*}^{\prime},-\infty p_{1}\right] U_{y}^{\dagger}\right\}+\text { a.c }\right] \mid P, S\right\rangle\right\rangle \\
= & \frac{s}{2}\left(\lambda \tilde{q}_{5 L}\left(k_{\perp}^{2}, x\right)-\frac{(S, k)_{\perp}}{M} \tilde{q}_{5 T}\left(k_{\perp}^{2}, x\right)\right) .
\end{aligned}
$$

We can also parametrize the matrix elements with the operators $\mathcal{Q}_{1 x}$ and $\mathcal{Q}_{5 x}$ as

$$
\begin{gathered}
\left.\int d^{2} \Delta e^{i(\Delta, k)}\left\langle\langle P, S|\left[\operatorname{Tr}\left\{\mathcal{Q}_{1}\left(x_{\perp}\right) U_{y}^{\dagger}\right\}+\text { a.c }\right] \mid P, S\right\rangle\right\rangle \\
=\frac{s}{2}\left(Q_{1}\left(k_{\perp}^{2}, x\right)+\frac{\vec{S} \times \vec{k}}{M} Q_{1 T}\left(k_{\perp}^{2}, x\right)\right) .
\end{gathered}
$$

and

$$
\begin{aligned}
\int d^{2} & \left.\Delta e^{i(\Delta, k)}\left\langle\langle P, S| \operatorname{Tr}\left\{\mathcal{Q}_{5}\left(x_{\perp}\right) U_{y}^{\dagger}\right\}+\text { a.c } \mid P, S\right\rangle\right\rangle \\
= & \frac{s}{2}\left(\lambda Q_{5 L}\left(k_{\perp}^{2}, x\right)+\frac{(S, k)_{\perp}}{M} Q_{1 T}\left(k_{\perp}^{2}, x\right)\right) .
\end{aligned}
$$

Using eqs. (4.26)-(4.29) we can find the following relations

$$
\begin{gathered}
2 Q_{1}\left(k_{\perp}^{2}, x\right)=q_{1}\left(k_{\perp}^{2}, x\right)-\frac{1}{N_{c}} \tilde{q}_{1}\left(k_{\perp}^{2}, x\right), \\
2 Q_{1 T}\left(k_{\perp}^{2}, x\right)=q_{1 T}\left(k_{\perp}^{2}, x\right)-\frac{1}{N_{c}} \tilde{q}_{1 T}\left(k_{\perp}^{2}, x\right), \\
2 Q_{5 L}\left(k_{\perp}^{2}, x\right)=q_{5 L}\left(k_{\perp}^{2}, x\right)-\frac{1}{N_{c}} \tilde{q}_{5 L}\left(k_{\perp}^{2}, x\right), \\
2 Q_{5 T}\left(k_{\perp}^{2}, x\right)=q_{5 T}\left(k_{\perp}^{2}, x\right)-\frac{1}{N_{c}} \tilde{q}_{5 T}\left(k_{\perp}^{2}, x\right) .
\end{gathered}
$$

The matrix element with the $\epsilon^{i j} F_{i j}\left(x_{*}, x_{\perp}\right)$ term is

$$
\begin{aligned}
& \left.\int d^{2} \Delta e^{i(\Delta, k)_{\perp}} \int_{-\infty}^{+\infty} d z_{*}\left\langle\langle P, S|\left[\operatorname{Tr}\left\{\left[\infty p_{1}, z_{*}\right]_{x} i g \frac{s}{2} \epsilon^{i j} F_{i j}\left(z_{*}, x_{\perp}\right)\left[z_{*},-\infty p_{1}\right]_{x} U_{y}^{\dagger}\right\}+\text { a.c }\right] \mid P, S\right\rangle\right\rangle \\
& =\frac{s}{2}\left[\lambda \frac{k_{\perp}^{2}}{M^{2}} G_{L}\left(k_{\perp}^{2}, x\right)+\frac{(S, k)_{\perp}}{M} G_{T}\left(k_{\perp}^{2}, x\right)\right]
\end{aligned}
$$

with helicity $\lambda= \pm \frac{1}{2}$. In $(5.13), G_{L}\left(k_{\perp}^{2}, x\right)$ and $G_{T}\left(k_{\perp}^{2}, x\right)$ are the polarized longitudinal and transverse gluon distributions of dimension $\left[M^{-2}\right]$ (see appendix $\mathrm{C}$ for details). Note that the parameterizations (5.3)-(5.13) are similar to the standard parameterizations for TMD [51-53]. 


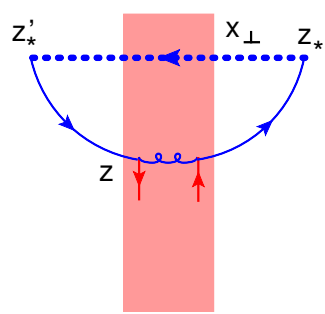

a)

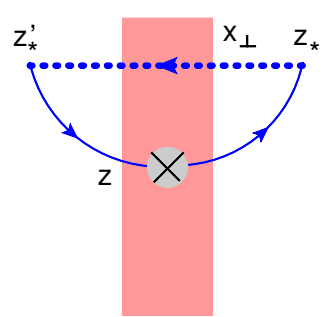

b)

Figure 5. Diagrams with $\hat{Q}_{1 x}$ and $\hat{Q}_{5 x}$ quantum.

\section{Evolution equation of sub-eikonal corrections}

In the eikonal approximation, which was presented in section 2, we first derived the LO impact factor and its associated dipole operator $\operatorname{Tr}\left\{U\left(x_{\perp}\right) U^{\dagger}\left(y_{\perp}\right)\right\}$, eq. (2.16), and then we proceeded with the calculation of the evolution of the dipole operator thus obtaining the BK equation. The plan is to repeat the same steps at sub-eikonal level. In the previous sections we derived the sub-eikonal impact factors and their associated operators. Our task is now the calculation of the evolution equations of these operators.

We use again the background field method. We have to separate fields in quantum and classical and perform the functional integration over the quantum fields leaving untouched the classical one. As a result of this procedure we will obtain a relation between the operator at the starting renormalization point $\eta_{1}$ and the new operators at the end renormalization point $\eta_{2}$ convoluted with a coefficient, the evolution kernel, which is the result of the functional integration over the quantum fields living in the infinitesimal rapidity interval $\eta_{1}-\eta_{2}=\Delta \eta$. This is nothing but the application of the Wilsonian renormalization group. Here the evolution parameter $\eta$ is the rapidity of the fields which we use to discriminate between classical and quantum fields. Indeed, at high energies, fields are ordered in rapidity space therefore it is natural to use it as the evolution parameter and as factorization parameter for scattering amplitudes. This logic is equal to the one adopted in the Bjorken limit, where the fields are ordered according to their transverse momentum and the factorization parameter $\mu_{f}$ discriminates between classical and quantum fields according to their transverse momenta.

A feature of the BK equation (and of BFKL equation) is that it is free of ultra violet (UV) and infra red (IR) divergences. Moreover, in the limit of vanishing dipole size unitarity is restored. What we will observe in the evolution equations of sub-eikonal operators, is the absence of this property which can be translated into a double log of energy contribution of the type $\alpha_{s} \ln ^{2} \frac{\alpha_{1}}{\alpha_{2}}$ where $\alpha_{1}$ and $\alpha_{2}$ are the longitudinal momenta of the fields within the infinitesimal step in rapidity where the quantum fields live.

To find evolution equation, the first step is to separate the gluon and the quark files in quantum and classical: $A_{\mu} \rightarrow A_{\mu}^{c l}+A_{\mu}^{q}$ and $\psi \rightarrow \psi^{c l}+\psi^{q}$. With this separation, the operator, for example, $\operatorname{Tr}\left\{\hat{\mathcal{Q}}_{1 z_{2}} \hat{U}_{z_{1}}^{\dagger}\right\}$ will generate several terms which will turn into Feynman diagrams through functional integration over the quantum fields. If the fields 
of the sub-eikonal corrections are classical, they will be in the shock-wave and we have to use the eikonal quark and gluon propagators. If, instead, the fields of the sub-eikonal operators are quantum, then they will be outside the shock-wave and will be functionally integrated. In this case, the sub-eikonal correction will be provided by the quark and gluon propagators with sub-eikonal corrections.

The relation we are looking for is of the type

$$
\operatorname{Tr}\left\{\hat{\mathcal{Q}}_{1 z_{2}} \hat{U}_{z_{1}}^{\dagger}\right\}^{\eta_{1}}=\Delta \eta K \otimes \hat{\mathcal{O}}^{\eta_{2}}
$$

where $\hat{\mathcal{O}}^{\eta_{2}}$ is the, in principle yet unknown, operator which we will obtain after one loop evolution. The new operator is convoluted with the kernel $K$ which is the result of functional integration. If one is able to solve the evolution equation, then we can convolute the solution with the impact factor with suitable initial conditions. This procedure gives the DIS structure functions and their behavior at high energy.

One of the operators we need to consider is

$$
\operatorname{Tr}\left\{\hat{\mathcal{Q}}_{1 x} \hat{U}_{y}^{\dagger}\right\}=\frac{1}{2} \hat{Q}_{1 x} \operatorname{Tr}\left\{\hat{U}_{x} \hat{U}_{y}^{\dagger}\right\}-\frac{1}{2 N_{c}} \operatorname{Tr}\left\{\hat{\tilde{Q}}_{x} \hat{U}_{y}^{\dagger}\right\}
$$

We will calculate the evolution of the l.h.s. in the appendix, while in the next section we will consider separately the evolution of the two terms in the r.h.s. Similarly will be done for the operator with $\hat{\mathcal{Q}}_{5}\left(x_{\perp}\right)$. The reason we calculate the evolution equation of the r.h.s. for the two terms will be clear when we consider diagrams with $\hat{\mathcal{F}}\left(x_{\perp}\right)$ quantum (see figures 9 and 10).

\subsection{Diagrams with $\hat{Q}_{1 x}$ and $\hat{Q}_{5 x}$ quantum}

As we explained in the previous section, we have to split all field in quantum and classical. Here we consider the case in which the operators $Q_{1 x}$ and $Q_{5 x}$ are quantum. The diagrams are give in figure 5. Let us consider operator $\operatorname{Tr}\left\{U_{y}^{\dagger} U_{x}\right\} Q_{1 x}$ and diagram 5a where there are quark fields in the background. First notice that

$$
\left\langle\operatorname{Tr}\left\{U_{y}^{\dagger} U_{x}\right\} Q_{1 x}\right\rangle_{\text {Figure 5 }}=\operatorname{Tr}\left\{U_{y}^{\dagger} U_{x}\right\}\left\langle Q_{1 x}\right\rangle_{\text {Figure } 5}
$$

So for the moment we will consider only $Q_{1 x}$.

As usual we indicate the quantum fields with the superscript $q$. We have

$$
\left\langle Q_{1 x}\right\rangle_{\text {Figure } 5 a}=g^{2} \int_{-\infty}^{+\infty} d z_{*} \int_{-\infty}^{z_{*}} d z_{*}^{\prime}\left\langle\operatorname{tr}\left\{i \not p_{1} \psi^{q}\left(z_{*}, x_{\perp}\right)\left[z_{*}, z_{*}^{\prime}\right]_{x}^{\dagger} \bar{\psi}^{q}\left(z_{*}^{\prime}, x_{\perp}\right)\right\}\right\rangle
$$

We need the quark propagator in the background of quark filed given in eq. (3.8) and get

$$
\begin{aligned}
& \left\langle Q_{1 x}\right\rangle_{\text {Figure } 5 a}=\frac{\alpha_{s}}{4 \pi^{2}} \int_{0}^{+\infty} \frac{d \alpha}{\alpha} \int d^{2} z g^{2} \int_{-\infty}^{+\infty} d z_{1 *} \int_{-\infty}^{z_{1 *}} d z_{2 *} \\
& \times\left[\frac{1}{2} \operatorname{Tr}\left\{U_{x}^{\dagger} U_{z}\right\} \operatorname{tr}\left\{i \not p_{1} \frac{(\not x-\not)_{\perp} \gamma_{\perp}^{\mu}}{(x-z)_{\perp}^{2}} \psi\left(z_{1 *}, z_{\perp}\right)\left[z_{1 *}, z_{2 *}\right]_{z}^{\dagger} \bar{\psi}\left(z_{2 *}, z_{\perp}\right) \frac{\gamma_{\perp}^{\mu}(\not k-\not x)_{\perp}}{(x-z)_{\perp}^{2}}\right\}\right. \\
& -\frac{1}{2 N_{c}} \operatorname{Tr}\left\{U _ { x } ^ { \dagger } [ \infty p _ { 1 } , z _ { 1 * } ] _ { z } \operatorname { t r } \left\{i \not p_{1} \frac{(\not x-\not)_{\perp} \gamma_{\perp}^{\mu}}{(x-z)_{\perp}^{2}} \psi\left(z_{1 *}, z_{\perp}\right) \bar{\psi}\left(z_{2 *}, z_{\perp}\right)\right.\right. \\
& \left.\left.\left.\times \frac{\gamma_{\perp}^{\mu}(\not-\not \not)_{\perp}}{(x-z)_{\perp}^{2}}\right\}\left[z_{2 *},-\infty p_{2}\right]_{z}\right\}\right] .
\end{aligned}
$$


Now, use

$$
\operatorname{tr}\left\{\not p_{1}(\not x-\not)_{\perp} \gamma_{\perp}^{\mu} \psi\left(z_{1 *}\right) \bar{\psi}\left(z_{2 *}\right) \gamma_{\mu}^{\perp}(\not-\not x)_{\perp}\right\}=2(x-z)_{\perp}^{2} \operatorname{tr}\left\{\not p_{1} \psi\left(z_{1 *}\right) \bar{\psi}\left(z_{2 *}\right)\right\}
$$

and arrive at

$$
\begin{aligned}
\left\langle Q_{1 x}\right\rangle_{\text {Figure } 5 a}= & \frac{\alpha_{s}}{4 \pi^{2}} \int_{0}^{+\infty} \frac{d \alpha}{\alpha} \int \frac{d^{2} z}{(x-z)_{\perp}^{2}} g^{2} \int_{-\infty}^{+\infty} d z_{1 *} \int_{-\infty}^{z_{1 *}} d z_{2 *} \\
\times & {\left[\operatorname{Tr}\left\{U_{x}^{\dagger} U_{z}\right\} \operatorname{tr}\left\{i \not p_{1} \psi\left(z_{1 *}, z_{\perp}\right)\left[z_{1 *}, z_{2 *}\right]_{z}^{\dagger} \bar{\psi}\left(z_{2 *}, z_{\perp}\right)\right\}\right.} \\
& \left.-\frac{1}{N_{c}} \operatorname{Tr}\left\{U_{x}^{\dagger}\left[\infty p_{1}, z_{1 *}\right]_{z} \operatorname{tr}\left\{i \not p_{1} \psi\left(z_{1 *}, z_{\perp}\right) \bar{\psi}\left(z_{2 *}, z_{\perp}\right)\right\}\left[z_{2 *},-\infty p_{2}\right]_{z}\right\}\right]
\end{aligned}
$$

Using the definition of operators $\hat{Q}_{1 x}$ and $\hat{\tilde{Q}}_{1 x}$ we obtain

$$
\left\langle Q_{1 x}\right\rangle_{\text {Figure } 5 a}=\frac{\alpha_{s}}{4 \pi^{2}} \int_{0}^{+\infty} \frac{d \alpha}{\alpha} \int \frac{d^{2} z}{(x-z)_{\perp}^{2}}\left[\operatorname{Tr}\left\{U_{x}^{\dagger} U_{z}\right\} Q_{1 z}-\frac{1}{N_{c}} \operatorname{Tr}\left\{U_{x}^{\dagger} \tilde{Q}_{1 z}\right\}\right]
$$

The same diagram calculated for the polarized quark operator $Q_{5 x}$ is

$$
\begin{aligned}
\left\langle Q_{5 x}\right\rangle_{\text {Figure } 5 a} & =g^{2} \int_{-\infty}^{+\infty} d z_{*} \int_{-\infty}^{z_{*}} d z_{*}^{\prime}\left\langle\operatorname{tr}\left\{\gamma^{5} \not p_{1} \psi\left(z_{*}, x_{\perp}\right)\left[z_{*}, z_{*}^{\prime}\right]_{x}^{\dagger} \bar{\psi}\left(z_{*}^{\prime}, x_{\perp}\right)\right\}\right\rangle \\
& =\frac{\alpha_{s}}{4 \pi^{2}} \int_{0}^{+\infty} \frac{d \alpha}{\alpha} \int \frac{d^{2} z}{(x-z)_{\perp}^{2}}\left[\operatorname{Tr}\left\{U_{x}^{\dagger} U_{z}\right\} Q_{5 z}-\frac{1}{N_{c}} \operatorname{Tr}\left\{U_{x}^{\dagger} \tilde{Q}_{5 z}\right\}\right]
\end{aligned}
$$

where this time we used

$$
\operatorname{tr}\left\{\gamma^{5} \not p_{1}(\not x-\not)_{\perp} \gamma_{\perp}^{\mu} \psi\left(z_{1 *}\right) \bar{\psi}\left(z_{2 *}\right) \gamma_{\mu}^{\perp}(\not-\not x)_{\perp}\right\}=2(x-z)_{\perp}^{2} \operatorname{tr}\left\{\gamma^{5} \not p_{1} \psi\left(z_{1 *}\right) \bar{\psi}\left(z_{2 *}\right)\right\}
$$

Now we consider diagram $5 \mathrm{~b}$ and start again with $Q_{1 x}$. We have

$$
\begin{aligned}
\left\langle Q_{1 x}\right\rangle_{\text {Figure } 5 b} & =g^{2} \int_{-\infty}^{+\infty} d z_{*} \int_{-\infty}^{z_{*}} d z_{*}^{\prime}\left\langle\operatorname{tr}\left\{i \not p_{1} \psi\left(z_{*}, x_{\perp}\right)\left[z_{*}, z_{*}^{\prime}\right]_{x}^{\dagger} \bar{\psi}\left(z_{*}^{\prime}, x_{\perp}\right)\right\}\right\rangle \\
& =\frac{\alpha_{s}}{2 \pi^{2}} \int_{0}^{+\infty} \frac{d \alpha}{\alpha} \int d^{2} z \operatorname{Tr}\left\{U_{x}^{\dagger} \mathcal{F}_{z}\right\} \operatorname{tr}\left\{i \not p_{1} \frac{(\not x-\not)_{\perp}}{(x-z)_{\perp}^{2}} \not p_{2} \gamma^{5} \frac{(\not-\not x)_{\perp}}{(z-\omega)_{\perp}^{2}}\right\}=0
\end{aligned}
$$

where we used $\operatorname{tr}\left\{\not \not_{1}(\not x-\not)_{\perp} \not \not_{2} \gamma^{5}(\not-\not x)_{\perp}\right\}=(x-z)_{\perp}^{2} \operatorname{tr}\left\{\not \not_{1} \not \not_{2} \gamma^{5}\right\}=0$. So, we see that operators of different parity do not mix under evolution. For operator $\hat{Q}_{5 x}$ we have, instead

$$
\begin{aligned}
\left\langle Q_{5 x}\right\rangle_{\text {Figure } 5 b} & =g^{2} \int_{-\infty}^{+\infty} d z_{*} \int_{-\infty}^{z_{*}} d z_{*}^{\prime}\left\langle\operatorname{tr}\left\{\gamma^{5} \not p_{1} \psi\left(z_{*}, x_{\perp}\right)\left[z_{*}, z_{*}^{\prime}\right]_{x}^{\dagger} \bar{\psi}\left(z_{*}^{\prime}, x_{\perp}\right)\right\}\right\rangle \\
& =\frac{\alpha_{s}}{2 \pi^{2}} \int_{0}^{+\infty} \frac{d \alpha}{\alpha} \int \frac{d^{2} z}{(x-z)_{\perp}^{2}} \operatorname{Tr}\left\{U_{x}^{\dagger} \mathcal{F}_{z}\right\}
\end{aligned}
$$

So, for diagrams in figure 5 we have

$$
\begin{aligned}
\left\langle\operatorname{Tr}\left\{U_{y}^{\dagger} U_{x}\right\} Q_{1 x}\right\rangle_{\text {Figure 5 }}= & \frac{\alpha_{s}}{4 \pi^{2}} \int_{0}^{+\infty} \frac{d \alpha}{\alpha} \int d^{2} z \frac{\operatorname{Tr}\left\{U_{y}^{\dagger} U_{x}\right\}}{(x-z)_{\perp}^{2}} \\
& \times\left[\operatorname{Tr}\left\{U_{x}^{\dagger} U_{z}\right\} Q_{1 z}-\frac{1}{N_{c}} \operatorname{Tr}\left\{U_{x}^{\dagger} \tilde{Q}_{1 z}\right\}\right]
\end{aligned}
$$




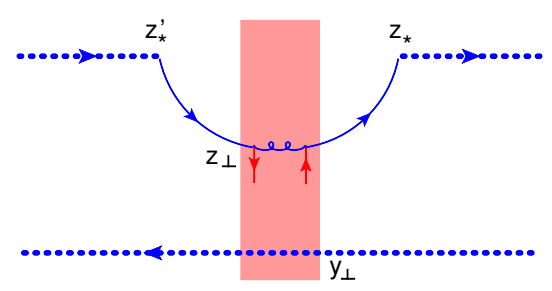

a)

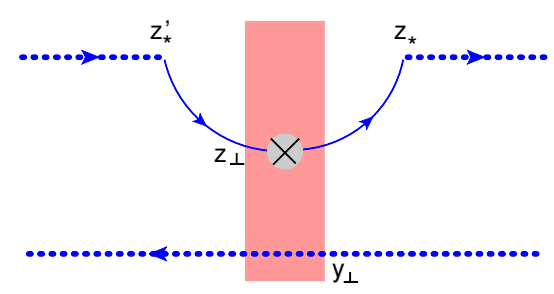

b)

Figure 6. Diagrams with $\tilde{Q}_{1 x}$ and $\tilde{Q}_{5 x}$ quantum.

and

$$
\begin{aligned}
\left\langle\operatorname{Tr}\left\{U_{y}^{\dagger} U_{x}\right\} Q_{5 x}\right\rangle_{\text {Figure 5 }}= & \frac{\alpha_{s}}{4 \pi^{2}} \int_{0}^{+\infty} \frac{d \alpha}{\alpha} \int d^{2} z \frac{\operatorname{Tr}\left\{U_{y}^{\dagger} U_{x}\right\}}{(x-z)_{\perp}^{2}} \\
& \times\left[\operatorname{Tr}\left\{U_{x}^{\dagger} U_{z}\right\} Q_{5 z}-\frac{1}{N_{c}} \operatorname{Tr}\left\{U_{x}^{\dagger}\left(\tilde{Q}_{5 z}-2 N_{c} \mathcal{F}_{z}\right)\right\}\right]
\end{aligned}
$$

\subsection{Diagrams with $\hat{\tilde{Q}}_{1 x}$ and $\hat{\tilde{Q}}_{5 x}$ quantum}

The diagrams with operators $\hat{\tilde{Q}}_{1 x}$ and $\hat{\tilde{Q}}_{5 x}$ are shown in figure 6 . Let us start with diagram with quark in the background. The calculation is similar to the diagrams in the previous section. We have

$$
\begin{aligned}
\left\langle\operatorname{Tr}\left\{U_{y}^{\dagger} \tilde{Q}_{1 x}\right\}\right\rangle_{\text {Figure } 6 a}= & g^{2} \int_{-\infty}^{+\infty} d z_{*} \int_{-\infty}^{z_{*}} d z_{*}^{\prime} \\
& \times\left\langle\operatorname{Tr}\left\{U_{y}^{\dagger}\left[\infty p_{1}, z_{*}\right]_{x} \operatorname{tr}\left\{i \not p_{1} \psi\left(z_{*}, x_{\perp}\right) \bar{\psi}\left(z_{*}^{\prime}, x_{\perp}\right)\right\}\left[z_{*}^{\prime},-p_{1} \infty\right]_{x}\right\}\right\rangle \\
= & g^{2} \int_{-\infty}^{+\infty} d z_{*} \int_{-\infty}^{z_{*}} d z_{*}^{\prime} \operatorname{Tr}\left\{U_{z}^{\dagger} \operatorname{tr}\left\{i \not p_{1}\left\langle\psi^{q}\left(z_{*}, x_{\perp}\right) \bar{\psi}^{q}\left(z_{*}^{\prime}, x_{\perp}\right)\right\rangle\right\}\right\}
\end{aligned}
$$

Now, using quark propagator in the background eq. (3.10) and performing Dirac algebra we get

$$
\left\langle\operatorname{Tr}\left\{U_{y}^{\dagger} \tilde{Q}_{1 x}\right\}\right\rangle_{\text {Figure } 6 a}=\frac{\alpha_{s}}{4 \pi^{2}} \int_{0}^{+\infty} \frac{d \alpha}{\alpha} \int \frac{d^{2} z}{(x-z)_{\perp}^{2}}\left[\operatorname{Tr}\left\{U_{y}^{\dagger} U_{z}\right\} Q_{1 z}-\frac{1}{N_{c}} \operatorname{Tr}\left\{U_{y}^{\dagger} \tilde{Q}_{1 z}\right\}\right]
$$

We se that after one loop we get mixing of operators $Q_{1 x}$ and $\tilde{Q}_{1 x}$.

For operator $\tilde{Q}_{5 x}$ we get the same result

$$
\left\langle\operatorname{Tr}\left\{U_{y}^{\dagger} \tilde{Q}_{5 x}\right\}\right\rangle_{\text {Figure } 6 a}=\frac{\alpha_{s}}{4 \pi^{2}} \int_{0}^{+\infty} \frac{d \alpha}{\alpha} \int \frac{d^{2} z}{(x-z)_{\perp}^{2}}\left[\operatorname{Tr}\left\{U_{y}^{\dagger} U_{z}\right\} Q_{5 z}-\frac{1}{N_{c}} \operatorname{Tr}\left\{U_{y}^{\dagger} \tilde{Q}_{5 z}\right\}\right]
$$

Next diagram is $6 \mathrm{~b}$. Starting with $\tilde{Q}_{1 x}$ we have

$$
\begin{aligned}
& \left\langle\operatorname{Tr}\left\{U_{y}^{\dagger} \tilde{Q}_{1 x}\right\}\right\rangle_{\text {Figure } 6 b} \\
& =g^{2} \int_{-\infty}^{+\infty} d z_{*} \int_{-\infty}^{z_{*}} d z_{*}^{\prime}\left\langle\operatorname{Tr}\left\{U_{z}^{\dagger}\left[\infty p_{1}, z_{*}\right]_{x} \operatorname{tr}\left\{i \not p_{1} \psi\left(z_{*}, x_{\perp}\right) \bar{\psi}\left(z_{*}^{\prime}, x_{\perp}\right)\right\}\left[z_{*}^{\prime},-p_{1} \infty\right]_{x}\right\}\right\rangle \\
& =g^{2} \int_{-\infty}^{+\infty} d z_{*} \int_{-\infty}^{z_{*}} d z_{*}^{\prime} \operatorname{Tr}\left\{U_{y}^{\dagger} \operatorname{tr}\left\{i \not p_{1}\left\langle\psi\left(z_{*}, x_{\perp}\right) \bar{\psi}\left(z_{*}^{\prime}, x_{\perp}\right)\right\rangle\right\}\right\} \\
& =\frac{\alpha_{s}}{8 \pi^{2}} \int_{0}^{+\infty} \frac{d \alpha}{\alpha} \int \frac{d^{2} z}{(x-z)_{\perp}^{4}} \frac{2}{s} \operatorname{Tr}\left\{U_{y}^{\dagger} \mathcal{F}_{z}\right\} \operatorname{tr}\left\{i \not p_{1}(\not x-\not)_{\perp} \not p_{2} \gamma^{5}(\not-\not \phi)_{\perp}\right\}=0
\end{aligned}
$$




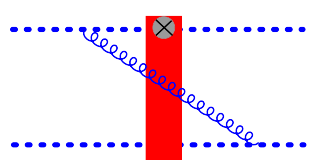

a)

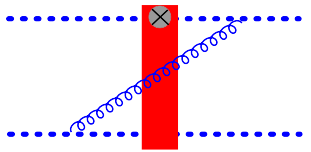

c)

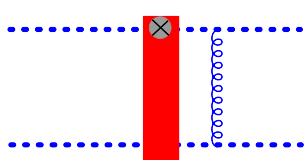

b)

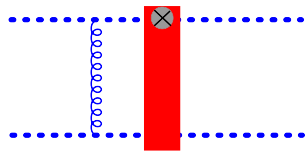

d)

Figure 7. Diagrams with sub-eikonal operator in the shock-wave. The gray circle with a cross on it represents the operator being treated as classical thus is situated in the red-band shock-wave.

As before, we get again no mixing between operators of different parity.

For operator $\tilde{Q}_{5 x}$ we have

$$
\begin{aligned}
& \left\langle\operatorname{Tr}\left\{U_{y}^{\dagger} \tilde{Q}_{5 x}\right\}\right\rangle_{\text {Figure } 6 b}=g^{2} \int_{-\infty}^{+\infty} d z_{*} \int_{-\infty}^{z_{*}} d z_{*}^{\prime} \operatorname{Tr}\left\{U_{y}^{\dagger} \operatorname{tr}\left\{\gamma^{5} \not p_{1}\left\langle\psi^{q}\left(z_{*}, x_{\perp}\right) \bar{\psi}^{q}\left(z_{*}^{\prime}, x_{\perp}\right)\right\rangle\right\}\right\} \\
& =\frac{\alpha_{s}}{8 \pi^{2}} \int_{0}^{+\infty} \frac{d \alpha}{\alpha} \int d^{2} z \frac{\operatorname{Tr}\left\{U_{y}^{\dagger} \mathcal{F}_{z}\right\}}{(x-z)_{\perp}^{4}} \frac{2}{s} \operatorname{tr}\left\{\gamma^{5} \not p_{1}(\not x-\not)_{\perp} \not p_{2} \gamma^{5}(\not z-\not x)_{\perp}\right\} \\
& =\frac{\alpha_{s}}{2 \pi^{2}} \int_{0}^{+\infty} \frac{d \alpha}{\alpha} \int \frac{d^{2} z}{(x-z)_{\perp}^{2}} \operatorname{Tr}\left\{U_{y}^{\dagger} \mathcal{F}_{z}\right\}
\end{aligned}
$$

We get, after one loop evolution, mixing between operator $\tilde{Q}_{5 x}$ and $\mathcal{F}_{x}$.

So, we conclude that

$$
\left\langle\operatorname{Tr}\left\{U_{y}^{\dagger} \tilde{Q}_{1 x}\right\}\right\rangle_{\text {Figure } 6}=\frac{\alpha_{s}}{4 \pi^{2}} \int_{0}^{+\infty} \frac{d \alpha}{\alpha} \int \frac{d^{2} z}{(x-z)_{\perp}^{2}}\left[\operatorname{Tr}\left\{U_{y}^{\dagger} U_{z}\right\} Q_{1 z}-\frac{1}{N_{c}} \operatorname{Tr}\left\{U_{y}^{\dagger} \tilde{Q}_{1 z}\right\}\right]
$$

and

$$
\begin{aligned}
\left\langle\operatorname{Tr}\left\{U_{y}^{\dagger} \tilde{Q}_{5 x}\right\}\right\rangle_{\text {Figure } 6}= & \frac{\alpha_{s}}{4 \pi^{2}} \int_{0}^{+\infty} \frac{d \alpha}{\alpha} \int \frac{d^{2} z}{(x-z)_{\perp}^{2}} \\
& \times\left[\operatorname{Tr}\left\{U_{y}^{\dagger} U_{z}\right\} Q_{5 z}-\frac{1}{N_{c}} \operatorname{Tr}\left\{U_{y}^{\dagger}\left(\tilde{Q}_{5 z}-2 N_{c} \mathcal{F}_{z}\right)\right\}\right]
\end{aligned}
$$

\subsection{BK-type diagrams}

The diagrams in figure 7 and 8 are similar to the BK diagrams in figure 2 with the only exception that in the shock-wave there is located a sub-eikonal correction that can be either $Q_{1}, \tilde{Q}_{1}, Q_{5}, \tilde{Q}_{5}$ (and their adjoint conjugated) or $\epsilon^{i j} F_{i j}$ (and in the appendix we will consider also $\mathcal{Q}_{1}$, or $\mathcal{Q}_{5}$ ).

As we will shortly see, the diagrams with $\tilde{Q}_{1 x}, \tilde{Q}_{1 x}$ (and their adjoint conjugated) and $\mathcal{F}_{x}$, are actually different then those with $Q_{1 x}$, and $Q_{5 x}$ (and their adjoint conjugated). So, we will calculate them separately. 


\subsubsection{BK-type diagrams for $Q_{1 x}$ and $Q_{5 x}$}

The BK-type of diagram for evolution for $\operatorname{Tr}\left\{U_{y}^{\dagger} U_{x}\right\} Q_{1 x}$ (or $\operatorname{Tr}\left\{U_{y}^{\dagger} U_{x}\right\} Q_{5 x}$ ) is

$$
\begin{aligned}
\left\langle Q_{1 x} \operatorname{Tr}\left\{U_{x} U_{y}^{\dagger}\right\}\right\rangle_{\text {Figures } 7+8}= & Q_{1 x}\left\langle\operatorname{Tr}\left\{U_{x} U_{y}^{\dagger}\right\}\right\rangle_{\text {Figures }} 7+8 \\
= & \frac{\alpha_{s}}{2 \pi^{2}} \int_{0}^{+\infty} \frac{d \alpha}{\alpha} \int d^{2} z \frac{(x-y)_{\perp}^{2}}{(x-z)_{\perp}^{2}(y-z)_{\perp}^{2}} \\
& \times\left[\operatorname{Tr}\left\{U_{x} U_{z}^{\dagger}\right\} \operatorname{Tr}\left\{U_{z} U_{y}^{\dagger}\right\}-N_{c} \operatorname{Tr}\left\{U_{x} U_{y}^{\dagger}\right\}\right] Q_{1 x}
\end{aligned}
$$

We get the same result if we replace $Q_{1 x}$ with $Q_{5 x}$. In the liner regime, evolution equation (6.22) gives the resummation in the LLA for the light-cone quark operator. However, in the double $\log$ of energy approximation, where $\alpha_{s} \ln ^{2}\left(1 / x_{B}\right) \sim 1$ and $\alpha_{s} \ln \left(1 / x_{B}\right) \ll 1$, contribution to the evolution equation $Q_{1 x}$ and $Q_{5 x}$ coming from (6.22) can be neglected.

\subsubsection{BK-type diagrams with $\tilde{Q}_{1 x}, \tilde{Q}_{5 x}, \mathcal{F}_{x}$}

Let us start with the real diagrams in figure 7 in which the quantum gluon (in blue) goes through the shock-wave. The calculation is the same as the diagram for BK equation. Considering, for example, operator $\tilde{Q}_{1 x}$, we have

$$
\left\langle\operatorname{Tr}\left\{\tilde{Q}_{1 x} U_{z}^{\dagger}\right\}\right\rangle_{\text {Figure } 7 a}=\frac{\alpha_{s}}{\pi^{2}} \int_{0}^{+\infty} \frac{d \alpha}{\alpha} \int d^{2} z U_{z}^{b a} \operatorname{Tr}\left\{\tilde{Q}_{1 x} t^{a} U_{y}^{\dagger} t^{b}\right\} \frac{(y-z, z-x)_{\perp}}{(y-z)_{\perp}^{2}(z-x)_{\perp}^{2}}
$$

In eq. (6.23) we notice the divergence in the longitudinal momentum component $\frac{1}{\alpha}$. Similarly to what we did for the evolution of the trace of two Wilson lines, we will regulate this divergence with a rigid cut-off and performing the derivative with respect this rapidity parameter we will obtain the evolution equation. The calculation of diagram in figure 7 is performed by first splitting all the fields of the l.h.s. of eq. (6.23) in classical and quantum, then considering only the terms in which operator $\hat{Q}_{i j}^{\alpha \beta}$ is left classical, and contracting the gluon quantum fields using the propagator in the background of a shock-wave given in eq.

The virtual diagram is given in figure (7b). Following similar procedure as for the real ones we obtain

$$
\left\langle\operatorname{Tr}\left\{\tilde{Q}_{1 x} U_{y}^{\dagger}\right\}\right\rangle_{\text {Figure } 7 b}=-\frac{\alpha_{s}}{\pi^{2}} \int_{0}^{+\infty} \frac{d \alpha}{\alpha} \int d^{2} z U_{z}^{b a} \operatorname{Tr}\left\{\tilde{Q}_{1 x} t^{a} U_{y}^{\dagger} t^{b}\right\} \frac{(y-z, z-x)_{\perp}}{(x-z)_{\perp}^{2}(y-z)_{\perp}^{2}}
$$

Using the symmetry which relates the diagrams in figure 7 we can also obtain the result of the last two i.e. diagrams in figure $7 \mathrm{a}$ and $\mathrm{b}$. Summing all them up and working out the color factor we have

$$
\begin{aligned}
& \left\langle\operatorname{Tr}\left\{\tilde{Q}_{1 x} U_{y}^{\dagger}\right\}\right\rangle_{\text {Figure } 7}=2\left\langle\operatorname{Tr}\left\{\tilde{Q}_{1 x} U_{y}^{\dagger}\right\}\right\rangle_{\text {Figure } 7 a+b} \\
& =\frac{\alpha_{s}}{2 \pi^{2}} \int_{0}^{+\infty} \frac{d \alpha}{\alpha} \int d^{2} z\left[2 U_{z}^{b a}-2 U_{y}^{b a}\right] \operatorname{Tr}\left\{\tilde{Q}_{1 x} t^{a} U_{y}^{\dagger} t^{b}\right\} \frac{2(y-z, z-x)_{\perp}}{(y-z)_{\perp}^{2}(z-x)_{\perp}^{2}} \\
& =\frac{\alpha_{s}}{2 \pi^{2}} \int_{0}^{+\infty} \frac{d \alpha}{\alpha} \int d^{2} z \frac{2(y-z, z-x)_{\perp}}{(y-z)_{\perp}^{2}(z-x)_{\perp}^{2}}\left[\operatorname{Tr}\left\{\tilde{Q}_{1 x} U_{z}^{\dagger}\right\} \operatorname{Tr}\left\{U_{z} U_{y}^{\dagger}\right\}-N_{c} \operatorname{Tr}\left\{\tilde{Q}_{1 x} U_{y}^{\dagger}\right\}\right]
\end{aligned}
$$

Let us consider self energy diagrams in figure 8 . The result is

$$
\left\langle\operatorname{Tr}\left\{\tilde{Q}_{1 x} U_{y}^{\dagger}\right\}\right\rangle_{\text {Figure } 8 a+b+c}=\frac{\alpha_{s}}{2 \pi^{2}} \int_{0}^{+\infty} \frac{d \alpha}{\alpha} \int \frac{d^{2} z}{(x-z)^{2}}\left[2 U_{z}^{a b}-2 U_{y}^{a b}\right] \operatorname{Tr}\left\{t^{a} \tilde{Q}_{1 x} t^{b} U_{y}^{\dagger}\right\}
$$



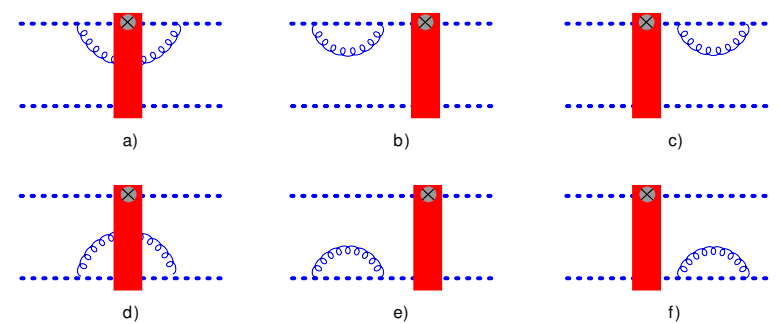

Figure 8. Self-energy diagrams with sub-eikonal correction inside and outside shock-wave.

and

$$
\left\langle\operatorname{Tr}\left\{\tilde{Q}_{1 x} U_{y}^{\dagger}\right\}\right\rangle_{\text {Figure } 8 d+e+f}=\frac{\alpha_{s}}{2 \pi^{2}} \int_{0}^{+\infty} \frac{d \alpha}{\alpha} \int d^{2} z\left[2 U_{z}^{a b}-2 U_{y}^{a b}\right] \operatorname{Tr}\left\{t^{a} \tilde{Q}_{1 x} t^{b} U_{y}^{\dagger}\right\} \frac{1}{(y-z)^{2}}
$$

Adding up the results for diagrams in figure 7 and 8 we have

$$
\begin{aligned}
\left\langle\operatorname{Tr}\left\{\tilde{Q}_{1 x} U_{y}^{\dagger}\right\}\right\rangle_{\text {Figure } 7+8}= & \frac{\alpha_{s}}{2 \pi^{2}} \int_{0}^{+\infty} \frac{d \alpha}{\alpha} \int d^{2} z \frac{(x-y)_{\perp}^{2}}{(x-z)_{\perp}^{2}(y-z)_{\perp}^{2}}\left[2 U_{z}^{a b}-2 U_{y}^{a b}\right] \operatorname{Tr}\left\{t^{a} \tilde{Q}_{1 x} t^{b} U_{y}^{\dagger}\right\} \\
= & \frac{\alpha_{s}}{2 \pi^{2}} \int_{0}^{+\infty} \frac{d \alpha}{\alpha} \int d^{2} z \frac{(x-y)_{\perp}^{2}}{(x-z)_{\perp}^{2}(y-z)_{\perp}^{2}} \\
& \times\left[\operatorname{Tr}\left\{U_{z}^{\dagger} \tilde{Q}_{1 x}\right\} \operatorname{Tr}\left\{U_{y}^{\dagger} U_{z}\right\}-N_{c} \operatorname{Tr}\left\{U_{y}^{\dagger} \tilde{Q}_{1 x}\right\}\right]
\end{aligned}
$$

Replacing operator $\hat{Q}_{1 x}$ with $\mathcal{Q}_{1 x}, \hat{\tilde{Q}}_{5 x}$, and $\mathcal{F}_{x}$ in eq. (6.28), we get the respective results for diagrams in figure 7 and 8 .

What should be noted in eq. (6.28) is that the unitarity property for vanishing dipole size is absent because in this limit the term $\operatorname{Tr}\left\{U_{z}^{\dagger} \tilde{Q}_{1 x}\right\} \operatorname{Tr}\left\{U_{y}^{\dagger} U_{z}\right\}$ does not reduce to $N_{c} \operatorname{Tr}\left\{U_{y}^{\dagger} \tilde{Q}_{1 x}\right\}$ when $z \rightarrow x$. This will be source of $\alpha_{s} \ln ^{2} \frac{1}{x_{B}}$ contributions [5].

\subsection{Evolution equation with $\epsilon^{i j} F_{i j}$ quantum}

In this section we consider the case in which the gluon operator $\mathcal{F}$ is quantum and, for this reason, it will be integrated out via functional integration. The diagrams for this case are shown in figure 9 and in figure 10.

In the Diagrams in figure 9 we take the gluon propagator with sub-eikonal correction due to gluon field. Let us start with diagram in figure 9a. We have

$$
\begin{aligned}
\langle\operatorname{Tr} & \left.\left\{g \int_{-\infty}^{+\infty} d x_{*}\left[\infty p_{1}, x_{*}\right]_{x} \epsilon^{i j} F_{i j}\left[x_{*},-\infty p_{1}\right]_{x} U_{y}^{\dagger}\right\}\right\rangle_{\text {Figure } 9 a} \\
& =-i g^{2} \int_{-\infty}^{0} d x_{*} \int_{0}^{+\infty} d \frac{2}{s} y_{*} \operatorname{Tr}\left\{U_{x} t^{a} U_{y}^{\dagger} t^{b}\right\}\left\langle A_{\bullet}^{b}\left(y_{*}, y_{\perp}\right) \epsilon^{i j} F_{i j}^{a}\left(x_{*}, x_{\perp}\right)\right\rangle
\end{aligned}
$$

From (6.29), we clearly see that $F_{i j}$ is now quantum and it will be integrated out. We will perform the calculation in the axial gauge and will use the gluon propagator (B.19). For the diagram under consideration the only terms that will survive the contraction of the 


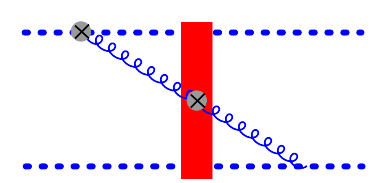

a)

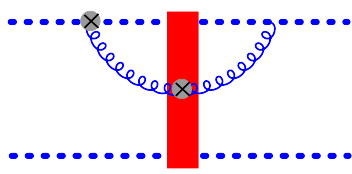

c)

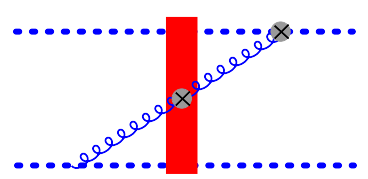

b)

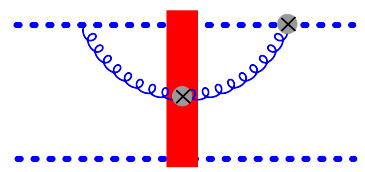

d)

Figure 9. Diagrams with $F_{i j}$ outside shock-wave. Here the gluon propagator, which represent the quantum loop, has gluon sub-eikonal corrections. The gray circle with an $\mathrm{x}$ on it symbolizes the $F_{i j}$ operator: it is quantum when it is on the Wilson-line and it is functionally integrated to create the gluon propagator; it is classical when it is in the shock-wave red band.

Lorentz indexes are the ones given in $\mathfrak{G}_{3}$, eq. (B.23), thus we have

$$
\begin{aligned}
& \left\langle\operatorname{Tr}\left\{g \int_{-\infty}^{+\infty} d x_{*}\left[\infty p_{1}, x_{*}\right]_{x} \epsilon^{i j} F_{i j}\left[x_{*},-\infty p_{1}\right]_{x} U_{y}^{\dagger}\right\}\right\rangle_{\text {Figure } 9 a} \\
& =-i g^{2} \int_{-\infty}^{0} d x_{*} \int_{0}^{+\infty} d \frac{2}{s} y_{*} \operatorname{Tr}\left\{U_{x} t^{a} U_{y}^{\dagger} t^{b}\right\}\left\langle A_{\bullet}^{b}\left(y_{*}, y_{\perp}\right) \epsilon^{i j} F_{i j}^{a}\left(x_{*}, x_{\perp}\right)\right\rangle \\
& =\frac{i g^{2}}{s^{2}} \operatorname{Tr}\left\{U_{x} t^{a} U_{y}^{\dagger} t^{b}\right\} \int_{0}^{+\infty} \frac{d \alpha}{\alpha^{3}} \int_{-\infty}^{0} d x_{*} \int_{0}^{+\infty} d y_{*} \epsilon^{i j}\left(\partial_{i}^{x} g_{j}^{l}-\partial_{j}^{x} g_{i}^{l}\right)\left\langle y_{\perp}\right| e^{-i \frac{\hat{p}_{\perp}^{2}}{\alpha s} y_{*}} \\
& \quad \times g \int_{-\infty}^{+\infty} d z_{*}\left[\left(\left[\infty p_{1}, z_{*}\right] i D^{k} F_{k l}\left[z_{*},-\infty p_{1}\right]\right)^{b a}\right. \\
& \left.\quad-2 g \int_{z_{*}}^{+\infty} d \frac{2}{s} z_{*}^{\prime}\left(\left[\infty p_{1}, z_{*}^{\prime}\right] F^{i} \bullet\left[z_{*}^{\prime}, z_{*}\right] F_{i j}\left[z_{*},-\infty p_{1}\right]\right)^{b a}\right] e^{i \frac{\hat{p}_{\perp}^{2}}{\alpha s} x_{*}}\left|x_{\perp}\right\rangle \\
& =-\frac{i g^{2}}{2 \pi} \operatorname{Tr}\left\{U_{x} t^{a} U_{y}^{\dagger} t^{b}\right\} \int_{0}^{+\infty} \frac{d \alpha}{\alpha} \int d^{2} z \epsilon^{i j}\left(\partial_{i}^{x} g_{j}^{l}-\partial_{j}^{x} g_{i}^{l}\right) \int d^{2} q_{1} \frac{e^{i\left(q_{1}, y-z\right)}}{q_{1 \perp}^{2}} \int d^{2} q_{2} \frac{e^{i\left(q_{2}, z-x\right)}}{q_{2 \perp}^{2}} \\
& \quad \times g \int_{-\infty}^{+\infty} d z_{*}\left[\left(\left[\infty p_{1}, z_{*}\right]_{z} i D^{k} F_{k l}\left[z_{*},-\infty p_{1}\right]_{z}\right)^{b a}\right. \\
& \left.\quad-2 g \int_{z_{*}}^{+\infty} d \frac{2}{s} z_{*}^{\prime}\left(\left[\infty p_{1}, z_{*}^{\prime}\right]_{z} F^{i} \bullet\left[z_{*}^{\prime}, z_{*}\right]_{z} F_{i j}\left[z_{*},-\infty p_{1}\right]_{z}\right)^{b a}\right]
\end{aligned}
$$

Now we consider diagram 9b. This time we need the sub-eikonal correction $\mathfrak{G}_{4}$, eq. (B.24) and obtain

$$
\begin{aligned}
& \left\langle\operatorname{Tr}\left\{g \int_{-\infty}^{+\infty} d x_{*}\left[\infty p_{1}, x_{*}\right]_{x} \epsilon^{i j} F_{i j}\left[x_{*},-\infty p_{1}\right]_{x} U_{y}^{\dagger}\right\}\right\rangle_{\text {Figure } 9 b} \\
& =-i g^{2} \int_{0}^{+\infty} d x_{*} \int_{-\infty}^{0} d \frac{2}{s} y_{*} \operatorname{Tr}\left\{t^{a} U_{x} t^{b} U_{y}^{\dagger}\right\}\left\langle F_{i j}^{a}\left(x_{*}, x_{\perp}\right) A_{\bullet}^{b}\left(y_{*}, y_{\perp}\right) \epsilon^{i j}\right\rangle \\
& =-\frac{i g^{2}}{2 \pi} \operatorname{Tr}\left\{t^{a} U_{x} t^{b} U_{y}^{\dagger}\right\} \int_{0}^{+\infty} \frac{d \alpha}{\alpha} \int d^{2} z \epsilon^{i j}\left(\partial_{i}^{x} g_{j}^{l}-\partial_{j}^{x} g_{i}^{l}\right) \int d^{2} q_{1} \frac{e^{i\left(q_{1}, x-z\right)}}{q_{1 \perp}^{2}} \int d^{2} q_{2} \frac{e^{i\left(q_{2}, z-y\right)}}{q_{2 \perp}^{2}} \\
& \quad \times g \int_{-\infty}^{+\infty} d z_{*}\left[\left(\left[\infty p_{1}, z_{*}\right]_{z} i D^{k} F_{k l}\left[z_{*},-\infty p_{1}\right]_{z}\right)^{a b}\right. \\
& \left.\quad-2 g \int_{z_{*}}^{+\infty} d \frac{2}{s} z_{*}^{\prime}\left(\left[\infty p_{1}, z_{*}^{\prime}\right]_{z} F_{i j}\left[z_{*}^{\prime}, z_{*}\right]_{z} F_{\bullet}^{i} \cdot\left[z_{*},-\infty p_{1}\right]_{z}\right)^{a b}\right]
\end{aligned}
$$


In summing diagrams $9 \mathrm{a}$ and $9 \mathrm{~b}$, we observe that the term with $D^{k} F_{k l}$ doubles and we may use the identity

$$
\begin{aligned}
2 g \int_{-\infty}^{+\infty} d z_{*}\left[\hat{P}^{k},\left(\left[\infty p_{1}, z_{*}\right]_{z} F_{k l}\left[z_{*},-\infty p_{1}\right]_{z}\right)^{b a}\right] \\
=2 g \int_{-\infty}^{+\infty} d z_{*} i \mathfrak{D}_{z}^{k}\left(\left[\infty p_{1}, z_{*}\right]_{z} F_{k l}\left[z_{*},-\infty p_{1}\right]_{z}\right)^{b a} \\
=2 g \int_{-\infty}^{+\infty} d z_{*}\left[\left(\left[\infty p_{1}, z_{*}\right]_{z} i D^{k} F_{k l}\left[z_{*},-\infty p_{1}\right]_{z}\right)^{b a}\right. \\
-g \int_{z_{*}}^{+\infty} d \frac{2}{s} z_{*}^{\prime}\left(\left[\infty p_{1}, z_{*}^{\prime}\right]_{z} F^{i} \cdot\left[z_{*}^{\prime}, z_{*}\right]_{z} F_{i j}\left[z_{*},-\infty p_{1}\right]_{z}\right)^{b a} \\
\left.-g \int_{z_{*}}^{+\infty} d \frac{2}{s} z_{*}^{\prime}\left(\left[\infty p_{1}, z_{*}^{\prime}\right]_{z} F_{i j}\left[z_{*}^{\prime}, z_{*}\right]_{z} F_{\bullet}^{i}\left[z_{*},-\infty p_{1}\right]_{z}\right)^{b a}\right]
\end{aligned}
$$

where in the first step we substituted the covariant derivative acting on gauge links and which is defined in eqs. (A.7) and (A.11). Furthermore, we observe that the operator $P^{i}=p^{i}+g A^{i}$ can be traded with $p^{i}$ because, as usual in these cases, the transverse gauge field is zero at the points outside the shock-wave.

So, using (6.32), the sum of diagrams $9 \mathrm{a}$ and $9 \mathrm{~b}$ is

$$
\begin{aligned}
&\left\langle\operatorname{Tr}\left\{g \int_{-\infty}^{+\infty} d x_{*}\left[\infty p_{1}, x_{*}\right]_{x} \epsilon^{i j} F_{i j}\left[x_{*},-\infty p_{1}\right]_{x} U_{y}^{\dagger}\right\}\right\rangle_{\text {Figure } 9 a+b} \\
&=\frac{i g^{2}}{s^{2}} \operatorname{Tr}\left\{U_{x} t^{a} U_{y}^{\dagger} t^{b}\right\} \int_{0}^{+\infty} \frac{d \alpha}{\alpha^{3}} \int_{-\infty}^{0} d x_{*} \int_{0}^{+\infty} d u_{*} \epsilon^{i j}\left(\partial_{i}^{x} g_{j}^{l}-\partial_{j}^{x} g_{i}^{l}\right)\left\langle z_{\perp}\right| e^{-i \frac{\hat{p}_{\perp}^{2}}{\alpha s} u_{*}} \\
& \quad \times 2 g \int_{-\infty}^{+\infty} d z_{*}\left[\hat{P}^{k},\left(\left[\infty p_{1}, z_{*}\right] F_{k l}\left[z_{*},-\infty p_{1}\right]\right)^{b a}\right] e^{i \frac{\hat{p}_{\perp}^{2}}{\alpha s} x_{*}\left|\omega_{\perp}\right\rangle} \\
&=-\frac{i g^{2}}{2 \pi} \operatorname{Tr}\left\{U_{x} t^{a} U_{y}^{\dagger} t^{b}\right\} \int_{0}^{+\infty} \frac{d \alpha}{\alpha} \int d^{2} z \epsilon^{i j}\left(i q_{2 i} g_{j}^{l}-i q_{2 j} g_{i}^{l}\right) \\
& \times \int d^{2} q_{1} \frac{e^{i\left(q_{1}, y-z\right)}}{q_{1 \perp}^{2}} \int d^{2} q_{2} \frac{e^{i\left(q_{2}, z-x\right)}}{q_{2 \perp}^{2}}\left(q_{1}^{k}-q_{2}^{k}\right) \\
& \times 2 g \int_{-\infty}^{+\infty} d z_{*}\left(\left[\infty p_{1}, z_{*}\right]_{z} F_{k l}\left[z_{*},-\infty p_{1}\right]_{z}\right)^{b a}
\end{aligned}
$$

Performing the Fourier transform we arrive at

$$
\begin{aligned}
\left\langle\operatorname{Tr}\left\{g \int_{-\infty}^{+\infty} d x_{*}\left[\infty p_{1}, x_{*}\right]_{x} \epsilon^{i j} F_{i j}\left(x_{*}, x_{\perp}\right)\left[x_{*},-\infty p_{1}\right]_{x} U_{y}^{\dagger}\right\}\right\rangle_{\text {Figure } 9 a+b} \\
=-\frac{g^{2}}{2 \pi} \operatorname{Tr}\left\{U_{x} t^{a} U_{y}^{\dagger} t^{b}\right\} \int_{0}^{+\infty} \frac{d \alpha}{\alpha} \int d^{2} z 2 g \int_{-\infty}^{+\infty} d z_{*}\left(\left[\infty p_{1}, z_{*}\right]_{z} \epsilon^{i j} F_{i j}\left[z_{*},-\infty p_{1}\right]_{z}\right)^{b a} \\
\quad \times\left[\frac{i(y-z)^{i}}{2 \pi(y-z)^{2}} \frac{i(z-x)^{i}}{2 \pi(z-x)^{2}}-\int d^{2} q_{1} \frac{e^{i\left(q_{1}, y-z\right)}}{q_{1 \perp}^{2}} \delta^{(2)}(z-x)\right]
\end{aligned}
$$

where we used $\epsilon^{i j}\left(i q_{2 i} F_{k j}-i q_{2 j} F_{k i}\right)\left(q_{1}^{k}-q_{2}^{k}\right)=-i \epsilon^{i j} F_{i j}\left(q_{2}, q_{1}-q_{2}\right)_{\perp}$.

At this point it should be clear why we performed all this massaging: the sum of diagrams $9 \mathrm{a}$ and $9 \mathrm{~b}$ gave back the same operator we started with, namely $\mathcal{F}\left(z_{\perp}\right)$ but in the adjoint representation. 
Let us consider the self energy diagrams in figure 9c and 9d. Integrating over the quantum field we have

$$
\begin{aligned}
\langle\operatorname{Tr} & \left.\left\{g \int_{-\infty}^{+\infty} d x_{*}\left[\infty p_{1}, x_{*}\right]_{x} \epsilon^{i j} F_{i j}\left[x_{*},-\infty p_{1}\right]_{x} U_{z}^{\dagger}\right\}\right\rangle_{\text {Figure } 9 c+d} \\
& =i g^{2} \frac{2}{s} \int_{-\infty}^{0} x_{*} \int_{0}^{+\infty} d x_{*}^{\prime} \operatorname{Tr}\left\{t^{a} U_{x} t^{b} U_{y}^{\dagger}\right\} \epsilon^{i j}\left(\partial_{i} g_{j}^{\nu}-\partial_{j} g_{i}^{\nu}\right)\left\langle A_{\bullet}^{a}\left(x_{*}^{\prime}, x_{\perp}\right) A_{\nu}^{b}\left(x_{*}, x_{\perp}\right)\right\rangle
\end{aligned}
$$

Repeating similar steps done for the real ones we arrive at

$$
\begin{gathered}
\left\langle\operatorname{Tr}\left\{g \int_{-\infty}^{+\infty} d x_{*}\left[\infty p_{1}, x_{*}\right]_{x} \epsilon^{i j} F_{i j}\left[x_{*},-\infty p_{1}\right]_{x} U_{z}^{\dagger}\right\}\right\rangle_{\text {Figure }} 9 c+d \\
=\frac{i g^{2}}{2 \pi} \operatorname{Tr}\left\{t^{a} U_{x} t^{b} U_{y}^{\dagger}\right\} \int_{0}^{+\infty} \frac{d \alpha}{\alpha} \epsilon^{i j}\left(i q_{2 i} g_{j}^{\nu}-i q_{2 j} g_{i}^{\nu}\right) \int d^{2} z \int d q_{1} \frac{e^{i\left(q_{1}, x-z\right)}}{q_{1 \perp}^{2}} \int d^{2} q_{2} \frac{e^{i\left(q_{2}, z-x\right)}}{q_{2 \perp}^{2}} \\
\times 2 g \int_{-\infty}^{+\infty} d z_{*}\left[\left(\left[\infty p_{1}, z_{*}\right]_{z} i D^{i} F_{i j}\left[z_{*},-\infty p_{1}\right]_{z}\right)^{a b}\right. \\
-g \frac{2}{s} \int_{z_{*}}^{+\infty} d z_{*}^{\prime}\left(\left[\infty p_{1}, z_{*}^{\prime}\right]_{z} F_{\bullet}^{l} \cdot\left[z_{*}^{\prime}, z_{*}\right]_{z} F_{l m}\left[z_{*},-\infty p_{1}\right]_{z}\right)^{a b} \\
\left.-g \frac{2}{s} \int_{z_{*}}^{+\infty} d z_{*}^{\prime}\left(\left[\infty p_{1}, z_{*}^{\prime}\right]_{z} F_{l m}\left[z_{*}^{\prime}, z_{*}\right]_{z} F_{\bullet}^{l}\left[z_{*},-\infty p_{1}\right]_{z}\right)^{a b}\right]
\end{gathered}
$$

and using again the identity (6.32) we obtain

$$
\begin{aligned}
\left\langle\operatorname{Tr}\left\{g \int_{-\infty}^{+\infty} d x_{*}\left[\infty p_{1}, x_{*}\right]_{x} \epsilon^{i j} F_{i j}\left[x_{*},-\infty p_{1}\right]_{x} U_{y}^{\dagger}\right\}\right\rangle_{\text {Figure } 9 c+d} \\
=-\frac{i g^{2}}{2 \pi} \operatorname{Tr}\left\{U_{x} t^{a} U_{y}^{\dagger} t^{b}\right\} \int_{0}^{+\infty} \frac{d \alpha}{\alpha} \int d^{2} z 2 g \int_{-\infty}^{+\infty} d z_{*}\left(\left[\infty p_{1}, z_{*}\right]_{z} F_{k l}\left[z_{*},-\infty p_{1}\right]_{z}\right)^{b a} \\
\quad \times \int d^{2} q_{1} \frac{e^{i\left(q_{1}, x-z\right)}}{q_{1 \perp}^{2}} \int d^{2} q_{2} \frac{e^{i\left(q_{2}, z-x\right)}}{q_{2 \perp}^{2}} \epsilon^{i j}\left(i q_{2 i} g_{j}^{l}-i q_{2 j} g_{i}^{l}\right)\left(q_{1}^{k}-q_{2}^{k}\right) .
\end{aligned}
$$

We can now perform the Fourier transform and add to it the result of the real diagrams and arrive at

$$
\begin{aligned}
& \left\langle\operatorname{Tr}\left\{\mathcal{F}_{x} U_{y}^{\dagger}\right\}\right\rangle_{\text {Figure } 9} \\
& =\frac{\alpha_{s}}{\pi^{2}} \int_{0}^{+\infty} \frac{d \alpha}{\alpha} \int d^{2} z \operatorname{Tr}\left\{U_{x} t^{a} U_{y}^{\dagger} t^{b}\right\} \mathcal{F}_{z}^{b a} \\
& \quad \times\left(\frac{(x-z, z-y)}{(y-z)_{\perp}^{2}(z-x)_{\perp}^{2}}+\frac{1}{(x-z)_{\perp}^{2}}+4 \pi^{2} \int d^{2} q_{1} \frac{e^{i\left(q_{1}, y-z\right)}-e^{i\left(q_{1}, x-z\right)}}{q_{1 \perp}^{2}} \delta^{(2)}(z-x)\right)
\end{aligned}
$$

where we defined the adjoint representation operator

$$
\mathcal{F}^{a b}\left(x_{\perp}\right)=i g \frac{s}{2} \int_{-\infty}^{+\infty} d z_{*}\left(\left[\infty p_{1}, z_{*}\right]_{x} \epsilon^{i j} F_{i j}\left(z_{*}, x_{\perp}\right)\left[z_{*},-\infty p_{1}\right]_{x}\right)^{a b} .
$$

Taking into consideration also quarks in the external field we get diagrams represented in figure 10. The gluon propagator with quarks in the external field given in eq. (B.25) has been calculated in ref. [46]. 


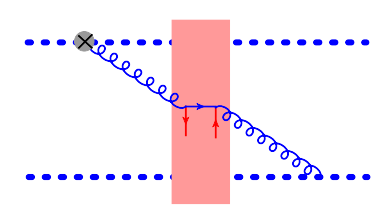

a)

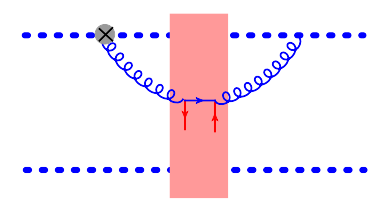

c)

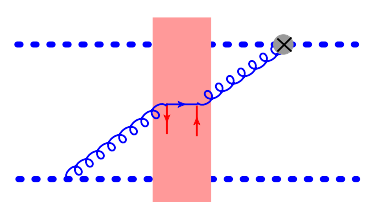

b)

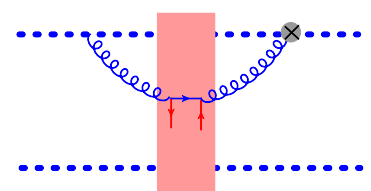

d)

Figure 10. Diagrams with $F_{i j}$ outside shock-wave. Here the gluon propagator, which represent the quantum loop, has quark sub-eikonal corrections.

We start with diagram in figure 10a and 10b

$$
\begin{aligned}
& \left\langle\operatorname{Tr}\left\{g \int_{-\infty}^{+\infty} d x_{*}\left[\infty p_{1}, x_{*}\right]_{x} \epsilon^{i j} F_{i j}\left[x_{*},-\infty p_{1}\right]_{x} U_{y}^{\dagger}\right\}\right\rangle_{\text {Figure } 10 a+b} \\
& =-i g^{2}\left[\int_{-\infty}^{0} d x_{*} \int_{0}^{+\infty} d \frac{2}{s} y_{*} \operatorname{Tr}\left\{U_{x} t^{a} U_{y}^{\dagger} t^{b}\right\}+\int_{0}^{+\infty} d x_{*} \int_{-\infty}^{0} d \frac{2}{s} y_{*} \operatorname{Tr}\left\{t^{a} U_{x} t^{b} U_{y}^{\dagger}\right\}\right] \\
& \quad \times\left\langle A_{\bullet}^{b}\left(y_{*}, y_{\perp}\right) \epsilon^{i j} F_{i j}^{a}\left(x_{*}, x_{\perp}\right)\right\rangle
\end{aligned}
$$

Using propagator (B.25) and performing simple spinor algebra we get the result for diagrams 10a and $10 \mathrm{~b}$

$$
\begin{aligned}
& \left\langle\operatorname{Tr}\left\{g \int_{-\infty}^{+\infty} d x_{*}\left[\infty p_{1}, x_{*}\right]_{x} \epsilon^{i j} F_{i j}\left(x_{*}, x_{\perp}\right)\left[x_{*},-\infty p_{1}\right]_{x} U_{y}^{\dagger}\right\}\right\rangle_{\text {Figure } 10 a+b} \\
& =4 \frac{g^{2}}{s} \operatorname{Tr}\left\{U_{x} t^{a} U_{y}^{\dagger} t^{b}\right\} \int_{0}^{+\infty} \frac{d \alpha}{\alpha} \int_{-\infty}^{+\infty} d z_{1 *} \int_{-\infty}^{z_{1 *}} d z_{2 *} \int d^{2} z \\
& \times g^{2}\left[\int \frac{d^{2} q_{1} d^{2} q_{2}}{q_{1 \perp}^{2} q_{2 \perp}^{2}} e^{i\left(q_{1}, y-z\right)+i\left(q_{2}, z-x\right)} \bar{\psi}\left(z_{1 *}, z_{\perp}\right)\left(\vec{q}_{2} \times \vec{q}_{1}+i\left(q_{1}, q_{2}\right) \gamma^{5}\right) \not p_{1}\right. \\
& \left.\times\left[z_{1 *}, \infty p_{1}\right]_{z} t^{b} U_{z} t^{a}\left[-\infty p_{1}, z_{2 *}\right]_{z} \psi\left(z_{2 *}, z_{\perp}\right)+\text { c.c. }\right] .
\end{aligned}
$$


Notice that we used the propagator (B.25) in the limit of $x^{+} \rightarrow+\infty$ and $y^{+} \rightarrow-\infty$ so that the gauge link gets $\left[x^{+}, y^{+}\right]_{z} \rightarrow U\left(z_{\perp}\right)$. After Fourier transform we have

$$
\begin{aligned}
\left\langle\operatorname{Tr}\left\{\mathcal{F}_{x} U_{y}^{\dagger}\right\}\right\rangle_{\text {Figure } 10 a+b} & \\
=-\frac{\alpha_{s}}{\pi^{2}} & \operatorname{Tr}\left\{U_{x} t^{a} U_{y}^{\dagger} t^{b}\right\} \int_{0}^{+\infty} \frac{d \alpha}{\alpha} \int d^{2} z \int_{-\infty}^{+\infty} d z_{1 *} \int_{-\infty}^{z_{1 *}} d z_{2 *} \\
\times g^{2}[ & \bar{\psi}\left(z_{1 *}, z_{\perp}\right)\left(\frac{(\vec{x}-\vec{z}) \times(\vec{z}-\vec{y})}{(x-z)_{\perp}^{2}(y-z)_{\perp}^{2}}+i \frac{(x-z, z-y) \gamma^{5}}{(x-z)_{\perp}^{2}(y-z)_{\perp}^{2}}\right) i \not p_{1} \\
& \times\left[z_{1 *}, \infty p_{1}\right]_{z} t^{b} U_{z} t^{a}\left[-\infty p_{1}, z_{2 *}\right]_{z} \psi\left(z_{2 *}, z_{\perp}\right) \\
& +\bar{\psi}\left(z_{2 *}, z_{\perp}\right)\left(\frac{(\vec{x}-\vec{z}) \times(\vec{z}-\vec{y})}{(x-z)_{\perp}^{2}(y-z)_{\perp}^{2}}+i \frac{(x-z, z-y) \gamma^{5}}{(x-z)_{\perp}^{2}(y-z)_{\perp}^{2}}\right) i \not p_{1} \\
& \left.\times\left[z_{2 *},-\infty p_{1}\right]_{z} t^{a} U_{z}^{\dagger} t^{b}\left[\infty p_{1}, z_{1 *}\right]_{z} \psi\left(z_{1 *}, z_{\perp}\right)\right]
\end{aligned}
$$

where we used again the two dimensional vector product as $\vec{x} \times \vec{y}=\epsilon^{i j} x_{i} y_{j}$.

Let us consider self energy diagrams in figure 10c and 10d. Proceeding in a similar way we obtain

$$
\begin{aligned}
& \left\langle\operatorname{Tr}\left\{g \int_{-\infty}^{+\infty} d x_{*}\left[\infty p_{1}, x_{*}\right]_{x} \epsilon^{i j} F_{i j}\left(x_{*}, x_{\perp}\right)\left[x_{*},-\infty p_{1}\right]_{x} U_{y}^{\dagger}\right\}\right\rangle_{\text {Figure } 10 c+d} \\
& =-\frac{\alpha_{s}}{\pi^{2}} \frac{2}{s} \operatorname{Tr}\left\{U_{x} t^{a} U_{y}^{\dagger} t^{b}\right\} \int_{0}^{+\infty} \frac{d \alpha}{\alpha} \int \frac{d^{2} z}{(x-z)_{\perp}^{2}} \int_{-\infty}^{+\infty} d z_{1 *} \int_{-\infty}^{z_{1 *}} d z_{2 *} \\
& \times g^{2}\left[\bar{\psi}\left(z_{1 *}, z_{\perp}\right) i \gamma^{5} \not p_{1}\left[z_{1 *}, \infty p_{1}\right]_{z} t^{b} U_{z} t^{a}\left[-\infty p_{1}, z_{2 *}\right]_{z} \psi\left(z_{2 *}, z_{\perp}\right)\right. \\
& \left.\quad+\bar{\psi}\left(z_{2 *}, z_{\perp}\right) i \gamma^{5} \not p_{1}\left[z_{2 *},-\infty p_{1}\right]_{z} t^{a} U_{z}^{\dagger} t^{b}\left[\infty p_{1}, z_{1 *}\right]_{z} \psi\left(z_{1 *}, z_{\perp}\right)\right]
\end{aligned}
$$

Let us define the following quark parity odd operators

$$
\begin{aligned}
& \mathcal{Q}_{5}^{a b}\left(z_{\perp}\right) \\
& \quad \equiv g^{2} \int_{-\infty}^{+\infty} d z_{1 *} \int_{-\infty}^{z_{1 *}} d z_{2 *} \bar{\psi}\left(z_{1 *}, z_{\perp}\right) \gamma^{5} \not p_{1}\left[z_{1 *}, \infty p_{1}\right]_{z} t^{a} U_{z} t^{b}\left[-\infty p_{1}, z_{2 *}\right]_{z} \psi\left(z_{2 *}, z_{\perp}\right), \\
& \mathcal{Q}_{5}^{a b{ }_{5}^{\dagger}\left(z_{\perp}\right)} \\
& \quad \equiv g^{2} \int_{-\infty}^{+\infty} d z_{1 *} \int_{-\infty}^{z_{1 *}} d z_{2 *} \bar{\psi}\left(z_{2 *}, z_{\perp}\right) \gamma^{5} \not p_{1}\left[z_{2 *},-\infty p_{1}\right]_{z} t^{b} U_{z}^{\dagger} t^{a}\left[\infty p_{1}, z_{1 *}\right]_{z} \psi\left(z_{1 *}, z_{\perp}\right)
\end{aligned}
$$

and parity even operators

$$
\begin{aligned}
& \mathcal{Q}_{1}^{a b}\left(z_{\perp}\right) \\
& \quad \equiv g^{2} \int_{-\infty}^{+\infty} d z_{1 *} \int_{-\infty}^{z_{1 *}} d z_{2 *} \bar{\psi}\left(z_{1 *}, z_{\perp}\right) i \not p_{1}\left[z_{1 *}, \infty p_{1}\right]_{z} t^{a} U_{z} t^{b}\left[-\infty p_{1}, z_{2 *}\right]_{z} \psi\left(z_{2 *}, z_{\perp}\right), \\
& \mathcal{Q}^{a b}{ }_{1}^{\dagger}\left(z_{\perp}\right) \\
& \quad \equiv-g^{2} \int_{-\infty}^{+\infty} d z_{1 *} \int_{-\infty}^{z_{1 *}} d z_{2 *} \bar{\psi}\left(z_{2 *}, z_{\perp}\right) i \not p_{1}\left[z_{2 *},-\infty p_{1}\right]_{z} t^{b} U_{z}^{\dagger} t^{a}\left[\infty p_{1}, z_{1 *}\right]_{z} \psi\left(z_{1 *}, z_{\perp}\right) .
\end{aligned}
$$


As usual, we will use the short-hand notation $\mathcal{Q}_{1 z}=\mathcal{Q}_{1}\left(z_{\perp}\right)$. Summing diagrams of figure 10 and using definitions (6.44)-(6.47) we have

$$
\begin{gathered}
\left\langle\operatorname{Tr}\left\{\mathcal{F}_{x} U_{y}^{\dagger}\right\}\right\rangle_{\text {Figure 10 }}=-\frac{\alpha_{s}}{\pi^{2}} \operatorname{Tr}\left\{U_{x} t^{a} U_{y}^{\dagger} t^{b}\right\} \int_{0}^{+\infty} \frac{d \alpha}{\alpha} \int d^{2} z\left[\frac{(\vec{x}-\vec{z}) \times(\vec{z}-\vec{y})}{(x-z)_{\perp}^{2}(y-z)_{\perp}^{2}}\left(\mathcal{Q}_{1 z}^{b a}-\mathcal{Q}_{1 z}^{b a \dagger}\right)\right. \\
\left.-\left(\frac{(x-z, z-y)}{(x-z)_{\perp}^{2}(y-z)_{\perp}^{2}}+\frac{1}{(x-z)_{\perp}^{2}}\right)\left(\mathcal{Q}_{5 z}^{b a}+\mathcal{Q}_{5 z}^{b a \dagger}\right)\right]
\end{gathered}
$$

The interesting thing to notice in result (6.48) is the appearance of operator $\mathcal{Q}_{1 x}^{a b}$ which although the operator itself is parity even it is multiplied by the $(\vec{x}-\vec{z}) \times(\vec{z}-\vec{y})$ so parity is preserved.

We can now sum up diagrams in figure 9 and 10 and obtain

$$
\begin{aligned}
&\left\langle\operatorname{Tr}\left\{\mathcal{F}_{x} U_{y}^{\dagger}\right\}\right\rangle_{\text {Figures }} 9+10 \\
&=-\frac{\alpha_{s}}{\pi^{2}} \operatorname{Tr}\left\{U_{x} t^{a} U_{y}^{\dagger} t^{b}\right\} \int_{0}^{+\infty} \frac{d \alpha}{\alpha} \int d^{2} z\left\{\frac{(\vec{x}-\vec{z}) \times(\vec{z}-\vec{y})}{(x-z)_{\perp}^{2}(y-z)_{\perp}^{2}}\left(\mathcal{Q}_{1 z}^{b a}-\mathcal{Q}_{1 z}^{b a \dagger}\right)\right. \\
&-\left(\frac{(x-z, z-y)}{(x-z)_{\perp}^{2}(y-z)_{\perp}^{2}}+\frac{1}{(x-z)_{\perp}^{2}}\right)\left(\mathcal{Q}_{5 z}^{b a}+\mathcal{Q}_{5 z}^{b a \dagger}+\mathcal{F}_{z}^{b a}\right) \\
&\left.-4 \pi^{2} \int d^{2} q_{1} \frac{e^{i\left(q_{1}, y-z\right)}-e^{i\left(q_{1}, x-z\right)}}{q_{1 \perp}^{2}} \delta^{(2)}(z-x) \mathcal{F}_{z}^{b a}\right\} .
\end{aligned}
$$

The first thing to notice in eq. (6.49) is that diagrams 9 and 10 have generated, after one loop evolution, operators $\hat{\mathcal{Q}}_{1}^{a b}\left(x_{\perp}\right), \hat{\mathcal{Q}}_{5}^{a b}\left(x_{\perp}\right)$, and $\hat{\mathcal{F}}^{a b}\left(x_{\perp}\right)$ which are not present in the OPE (4.36). This means that, either we have to find the evolution of this new operators or try to reduce them to the operators we started with i.e $\hat{\mathcal{F}}\left(x_{\perp}\right), \hat{\mathcal{Q}}_{1}\left(x_{\perp}\right)$ and $\hat{\mathcal{Q}}_{5}\left(x_{\perp}\right)$. Performing some color algebra we can reduce the r.h.s. of eq. (6.49) in the fundamental 

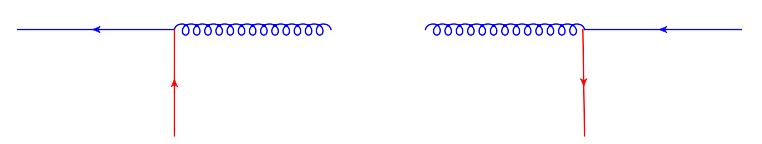

Figure 11. Diagrams for quark-to-gluon propagator.

representation

$$
\begin{aligned}
& \left\langle\operatorname{Tr}\left\{\mathcal{F}_{x} U_{y}^{\dagger}\right\}\right\rangle_{\text {Figures }} 9+10 \\
& =\frac{\alpha_{s}}{2 \pi^{2}} \int_{0}^{+\infty} \frac{d \alpha}{\alpha} \int d^{2} z\left\{\frac { 1 } { 2 } \frac { ( \vec { x } - \vec { z } ) \times ( \vec { z } - \vec { y } ) } { ( x - z ) _ { \perp } ^ { 2 } ( y - z ) _ { \perp } ^ { 2 } } \left[\operatorname{Tr}\left\{U_{y}^{\dagger} \tilde{Q}_{1 z}\right\} \operatorname{Tr}\left\{U_{z}^{\dagger} U_{x}\right\}-\operatorname{Tr}\left\{U_{x} \tilde{Q}_{1 z}^{\dagger}\right\} \operatorname{Tr}\left\{U_{y}^{\dagger} U_{z}\right\}\right.\right. \\
& +\frac{1}{N_{c}}\left(\operatorname{Tr}\left\{U_{x} U_{y}^{\dagger} \tilde{Q}_{1 z} U_{z}^{\dagger}\right\}+\operatorname{Tr}\left\{U_{y}^{\dagger} U_{x} U_{z}^{\dagger} \tilde{Q}_{1 z}\right\}-\operatorname{Tr}\left\{U_{x} U_{y}^{\dagger} U_{z} \tilde{Q}_{1 z}^{\dagger}\right\}-\operatorname{Tr}\left\{U_{y}^{\dagger} U_{x} \tilde{Q}_{1 z}^{\dagger} U_{z}\right\}\right) \\
& \left.+\frac{1}{N_{c}^{2}} \operatorname{Tr}\left\{U_{y}^{\dagger} U_{x}\right\}\left(Q_{1 z}^{\dagger}-Q_{1 z}\right)\right]-\frac{1}{2}\left[\frac{(x-z, z-y)}{(x-z)_{\perp}^{2}(y-z)_{\perp}^{2}}+\frac{1}{(x-z)_{\perp}^{2}}\right] \\
& \times\left[\operatorname{Tr}\left\{U_{y}^{\dagger}\left(\tilde{Q}_{5 z}-2 \mathcal{F}_{z}\right)\right\} \operatorname{Tr}\left\{U_{z}^{\dagger} U_{x}\right\}+\operatorname{Tr}\left\{U_{x}\left(\tilde{Q}_{5 z}^{\dagger}-2 \mathcal{F}_{z}^{\dagger}\right)\right\} \operatorname{Tr}\left\{U_{y}^{\dagger} U_{z}\right\}\right. \\
& -\frac{1}{N_{c}}\left(\operatorname{Tr}\left\{U_{x} U_{y}^{\dagger} U_{z} \tilde{Q}_{5 z}^{\dagger}\right\}+\operatorname{Tr}\left\{U_{y}^{\dagger} U_{x} \tilde{Q}_{5 z}^{\dagger} U_{z}\right\}+\operatorname{Tr}\left\{U_{x} U_{y}^{\dagger} \tilde{Q}_{5 z} U_{z}^{\dagger}\right\}+\operatorname{Tr}\left\{U_{y}^{\dagger} U_{x} U_{z}^{\dagger} \tilde{Q}_{5 z}\right\}\right) \\
& \left.+\frac{1}{N_{c}^{2}} \operatorname{Tr}\left\{U_{y}^{\dagger} U_{x}\right\}\left(Q_{5 z}+Q_{5 z}^{\dagger}\right)\right] \\
& +4 \pi^{2} \int d^{2} q_{1} \frac{e^{i\left(q_{1}, y-z\right)}-e^{i\left(q_{1}, x-z\right)}}{q_{1 \perp}^{2}} \delta^{(2)}(z-x) \\
& \left.\times\left[\operatorname{Tr}\left\{U_{x} U_{z}^{\dagger}\right\} \operatorname{Tr}\left\{U_{y}^{\dagger} \mathcal{F}_{z}\right\}+\operatorname{Tr}\left\{U_{x}^{\dagger} U_{z}\right\} \operatorname{Tr}\left\{U_{x} \mathcal{F}_{z}^{\dagger}\right\}\right]\right\}
\end{aligned}
$$

So, writing the evolution equation for $\operatorname{Tr}\left\{\mathcal{F}_{x} U_{y}^{\dagger}\right\}$ in the fundamental representation, we eliminated the operators $\hat{\mathcal{F}}_{x}^{a b}, \hat{\mathcal{Q}}_{1 x}^{a b}, \hat{\mathcal{Q}}_{5 x}^{a b}$ and their adjoint conjugated, in favor of operators $\hat{\mathcal{F}}_{x}, \hat{Q}_{1 x}, \hat{Q}_{5 x}, \hat{\tilde{Q}}_{1 x}, \hat{\widetilde{Q}}_{5 x}$ and their adjoint conjugated, defined in eqs. (4.18)-(4.25), that are present in the OPE (4.36) and (4.39).

From eq. (6.50) it should be clear why we decided to calculate the diagrams using operators $\hat{Q}_{1 x}, \hat{Q}_{5 x}, \hat{\tilde{Q}}_{1 x}, \hat{\tilde{Q}}_{5 x}$, instead of $\hat{\mathcal{Q}}_{1 x}$ and $\hat{\mathcal{Q}}_{5 x}$.

\subsection{Diagrams with quark-to-gluon propagator}

The diagrams we have calculated until now were obtained using the quark and gluon propagators originally calculated in reference [46] and summarized in appendix B.

In this section we are going to calculate the one-loop correction to the quark operators $\hat{Q}_{1 x}, \hat{Q}_{5 x}, \hat{\tilde{Q}}_{1 x}$, and $\hat{\tilde{Q}}_{5 x}$ defined in eqs. (4.18)-4.25 using the quark-to-gluon propagator shown in figure 11.

\subsubsection{Quark-to-gluon propagator}

First, let us calculate the quark-to-gluon propagator. We have two contributions represented in diagrams in figure 11. As usual, the quantum fields are indicated with the 
superscript (or subscript) $q$ while the classical ones are indicated with the superscript (or subscript) $c l$ although the superscript (or subscript) $c l$ will be eventually dropped.

We need the following quark propagators in the eikonal approximation

$$
\begin{aligned}
& \langle\psi(x) \bar{\psi}(y)\rangle \\
& =\left[\int_{0}^{+\infty} \frac{d \alpha}{2 \alpha} \theta\left(x_{*}-y_{*}\right)-\int_{-\infty}^{0} \frac{d \alpha}{2 \alpha} \theta\left(y_{*}-x_{*}\right)\right] e^{-i \alpha\left(x_{\bullet}-y_{\bullet}\right)} \frac{1}{\alpha s} \\
& \quad \times\left\langle x_{\perp}\left|e^{-i \frac{p_{\perp}^{2}}{\alpha s} x_{*}}\left(\left[x_{*}, y_{*}\right] \not p p p_{2} \not p-g \int_{y_{*}}^{x_{*}} d \frac{2}{s} \omega_{*}\left[x_{*}, \omega_{*}\right] F_{i \bullet}\left[\omega_{*}, y_{*}\right] \gamma^{i} \not p_{2} \not p\right) e^{i \frac{p_{\perp}^{2}}{\alpha s} y_{*}}\right| y_{\perp}\right\rangle
\end{aligned}
$$

and

$$
\begin{aligned}
& \langle\psi(x) \bar{\psi}(y)\rangle \\
& =\left[\int_{0}^{+\infty} \frac{d \alpha}{2 \alpha} \theta\left(x_{*}-y_{*}\right)-\int_{-\infty}^{0} \frac{d \alpha}{2 \alpha} \theta\left(y_{*}-x_{*}\right)\right] e^{-i \alpha\left(x_{\bullet}-y_{\bullet}\right)} \frac{1}{\alpha s} \\
& \quad \times\left\langle x_{\perp}\left|e^{-i \frac{\hat{p}_{\perp}^{2}}{\alpha s} x_{*}}\left(\hat{\not p h} \hat{p}_{2} \hat{\not p}\left[x_{*}, y_{*}\right]-g \int_{y_{*}}^{x_{*}} d \frac{2}{s} \omega_{*} \hat{p h} p_{2} \gamma^{i}\left[x_{*}, \omega_{*}\right] F_{i \bullet}\left[\omega_{*}, y_{*}\right]\right) e^{i \frac{\hat{p}_{\perp}^{2}}{\alpha s} y_{*}}\right| y_{\perp}\right\rangle .
\end{aligned}
$$

Notice that we are not using the usual eikonal quark propagator in the shock-wave which has structure free propagator-shock-wave-free propagator as given in the eikonal term of quark propagator in eq. (B.12). The transition from the eikonal propagators (6.51) and (6.52) to the eikonal part of the quark propagator (B.12) is explained ino ref. [46]. Here we just mention that with the help of eqs. (A.6), (A.7), and (A.11), we can show that the work propagators (6.52), and (6.51) can easily be put in the usual form

$$
\begin{aligned}
\left\langle x\left|\frac{i}{\not P+i \epsilon}\right| y\right\rangle= & \frac{1}{s}\left[\int_{0}^{+\infty} \frac{d \alpha}{2 \alpha^{2}} \theta\left(x_{*}-y_{*}\right)-\int_{-\infty}^{0} \frac{d \alpha}{2 \alpha^{2}} \theta\left(y_{*}-x_{*}\right)\right] e^{-i \alpha\left(x_{\bullet}-y_{\bullet}\right)} \\
& \times\left\langle x_{\perp}\left|e^{-i \frac{\hat{p}_{\perp}^{2}}{\alpha s} x_{*}} \not p\right| z_{\perp}\right\rangle \not p_{2} U_{z}\left\langle z_{\perp}\left|\not p e^{i \frac{\hat{p}_{\perp}^{2}}{\alpha s} y_{*}}\right| y_{\perp}\right\rangle .
\end{aligned}
$$

However, as we will soon see, to calculate diagrams in figure 11 we will need the form of the quark propagator given in (6.52), and (6.51).

We also need the eikonal gluon propagator in the axial gauge

$$
\begin{aligned}
\left\langle A_{\mu}^{a}(x) A_{\nu}^{b}(y)\right\rangle_{A}= & {\left[-\int_{0}^{+\infty} \frac{d \alpha}{2 \alpha} \theta\left(x_{*}-y_{*}\right)+\int_{-\infty}^{0} \frac{d \alpha}{2 \alpha} \theta\left(y_{*}-x_{*}\right)\right] e^{-i \alpha\left(x_{\bullet}-y_{\bullet}\right)} } \\
& \times\left\langle x_{\perp}\left|e^{-i \frac{\hat{p}_{\perp}^{2}}{\alpha s} x_{*}}\left(\delta_{\mu}^{\xi}-\frac{2}{s} \frac{p_{2 \mu}}{\alpha} p^{\xi}\right)\left[x_{*}, y_{*}\right]\left(g_{\xi \nu}-\frac{2}{s} p_{\xi} \frac{p_{2 \nu}}{\alpha}\right) e^{i \frac{\hat{p}_{\perp}^{2}}{\alpha s} y_{*}}\right| y_{\perp}\right\rangle^{a b}
\end{aligned}
$$


Using quark propagator (6.52) and gluon propagator (6.54), we can calculate the first diagram for the quark-to-gluon propagator

$$
\begin{aligned}
& \left\langle A_{\mu}^{a}(x) \bar{\psi}(y)\right\rangle=\left\langle A_{\mu}^{q, a}(x) \bar{\psi}^{q}(y) i g \int d^{4} z \bar{\psi}^{c l}(z) \mathcal{A}^{q}(z) \psi^{q}(z)\right\rangle \\
& =i g \int d^{4} z\left[-\int_{0}^{+\infty} \frac{d \alpha}{2 \alpha} \theta\left(x_{*}-z_{*}\right)+\int_{-\infty}^{0} \frac{d \alpha}{2 \alpha} \theta\left(z_{*}-x_{*}\right)\right] e^{-i \alpha\left(x_{\bullet}-z_{\bullet}\right)} \\
& \times\left\langle x_{\perp}\left|e^{-i \frac{\hat{p}_{\perp}^{2}}{\alpha s} x_{*}}\left(\delta_{\mu}^{\xi}-\frac{p_{2 \mu}}{p_{*}} p^{\xi}\right)\left[x_{*}, z_{*}\right]\left(g_{\xi \nu}-p_{\xi} \frac{p_{2 \nu}}{p_{*}}\right) e^{i \frac{\hat{p}_{\perp}^{2}}{\alpha s} z_{*}}\right| z_{\perp}\right\rangle^{a b} \bar{\psi}^{c l}(z) t^{b} \\
& \times\left[\int_{0}^{+\infty} \frac{d \alpha}{2 \alpha} \theta\left(z_{*}-y_{*}\right)-\int_{-\infty}^{0} \frac{d \alpha}{2 \alpha} \theta\left(y_{*}-z_{*}\right)\right] e^{-i \alpha\left(z_{\bullet}-y_{\bullet}\right)} \frac{1}{\alpha s} \\
& \times\left\langle z_{\perp}\left|e^{-i \frac{p_{\perp}^{2}}{\alpha s} z_{*}}\left(\not p p p p_{2} \not p\left[z_{*}, y_{*}\right]-g \int_{y_{*}}^{z_{*}} d \frac{2}{s} \omega_{*} \not p p p_{2} \gamma^{i}\left[z_{*}, \omega_{*}\right] F_{i \bullet}\left[\omega_{*}, y_{*}\right]\right) e^{i \frac{p_{\perp}^{2}}{\alpha s} y_{*}}\right| y_{\perp}\right\rangle \\
& =i g \int_{y_{*}}^{x_{*}} d \frac{2}{s} z_{*}\left[-\int_{0}^{+\infty} \frac{d \alpha}{4 \alpha^{2}} \theta\left(x_{*}-y_{*}\right)+\int_{-\infty}^{0} \frac{d \alpha}{4 \alpha^{2}} \theta\left(y_{*}-x_{*}\right)\right] e^{-i \alpha\left(x_{\bullet}-y_{\bullet}\right)} \int d^{2} z \\
& \times\left\langle x_{\perp}\left|e^{-i \frac{p_{\perp}^{2}}{\alpha s} x_{*}}\left(g_{\mu \nu}^{\perp}-\frac{2}{s} \frac{p_{\nu}^{\perp} p_{2 \mu}}{\alpha}\right)\right| z_{\perp}\right\rangle\left[x_{*}, z_{*}\right]_{z}^{a b} \bar{\psi}^{c l}\left(z_{*}, z_{\perp}\right) t^{b} \gamma_{\perp}^{\nu}\left[z_{*}, y_{*}\right]_{z} \\
& \times\left\langle z_{\perp}\left|\left(\alpha \not p_{1}+\not p_{\perp}\right) e^{i \frac{p_{\perp}^{2}}{\alpha s} y_{*}}\right| y_{\perp}\right\rangle+O\left(\lambda^{-2}\right)
\end{aligned}
$$

where we used $\not p_{2} \psi=\bar{\psi} \not p_{2}=O\left(\lambda^{-2}\right)$ and

$$
\left(\delta_{\mu}^{\nu}-\frac{p_{2 \mu} p_{\nu}}{p_{*}}\right)\left[x_{*}, z_{*}\right]^{a b} \bar{\psi}\left(z_{*}\right) \gamma^{\nu}=\left(g_{\perp \mu}^{\nu}-\frac{p_{2 \mu} p_{\perp \nu}}{p_{*}}\right)\left[x_{*}, z_{*}\right]^{a b} \bar{\psi}\left(z_{*}\right) \gamma_{\perp}^{\nu}+O\left(\lambda^{-2}\right)
$$

and

$$
\begin{aligned}
\left(\delta_{\mu}^{\nu}\right. & \left.-\frac{p_{2 \mu} p_{\nu}}{p_{*}}\right)\left[x_{*}, z_{*}\right]^{a b}\left(g_{\xi \nu}-p_{\xi} \frac{p_{2 \nu}}{p_{*}}\right) \bar{\psi}\left(z_{*}\right) \gamma^{\nu} \\
& =\left(g_{\perp \mu}^{\nu}-\frac{p_{2 \mu} p_{\perp \nu}}{p_{*}}\right)\left[x_{*}, z_{*}\right]^{a b} \bar{\psi}\left(z_{*}\right) \gamma_{\perp}^{\nu}+O\left(\lambda^{-2}\right)
\end{aligned}
$$

We remind the reader that the parameter $\lambda$ is the large boost-parameter which discriminates between the components of the fields (see appendix A for details and ref. [46]). Similarly, we have

$$
\begin{aligned}
\left\langle\psi(y) A_{\mu}^{a}(x)\right\rangle= & \left\langle\psi^{q}(y) A_{\mu}^{q, a}(x) i g \int d^{4} z \bar{\psi}^{q}(z) \mathcal{A}^{q}(z) \psi^{c l}(z)\right\rangle \\
= & i g \int_{x_{*}}^{y_{*}} d \frac{2}{s} z_{*}\left[-\int_{0}^{+\infty} \frac{d \alpha}{4 \alpha^{2}} \theta\left(y_{*}-x_{*}\right)+\int_{-\infty}^{0} \frac{d \alpha}{4 \alpha^{2}} \theta\left(x_{*}-y_{*}\right)\right] e^{-i \alpha\left(y_{\bullet}-x_{\bullet}\right)} \\
& \times \int d^{2} z\left\langle y_{\perp}\left|e^{-i \frac{p_{\perp}^{2}}{\alpha s} y_{*}}\left(\alpha \not p_{1}+\not p_{\perp}\right)\right| z_{\perp}\right\rangle\left[y_{*}, z_{*}\right]_{z} \gamma^{\nu} t^{b} \psi^{c l}\left(z_{*}, z_{\perp}\right)\left[z_{*}, x_{*}\right]_{z}^{b a} \\
& \times\left\langle z_{\perp}\left|\left(g_{\nu \mu}^{\perp}-\frac{2}{s} \frac{p_{\nu}^{\perp} p_{2 \mu}}{\alpha}\right) e^{i \frac{p_{\perp}^{2}}{\alpha s} x_{*}}\right| x_{\perp}\right\rangle+O\left(\lambda^{-2}\right)
\end{aligned}
$$




\subsubsection{Operators definition}

Before we start the calculation of the diagrams we defined operators that will be useful for the subsequent results. So, we define

$$
\begin{aligned}
& \mathcal{X}_{1}\left(x_{\perp}, y_{\perp}\right)=-g^{2} \int_{-\infty}^{+\infty} d z_{*} d \omega_{*} \bar{\psi}\left(z_{*}, y_{\perp}\right)\left[z_{*},-\infty p_{1}\right]_{y} i \not p_{1}\left[\infty p_{1}, \omega_{*}\right]_{x} \psi\left(\omega_{*}, x_{\perp}\right), \\
& \mathcal{X}_{1}^{\dagger}\left(x_{\perp}, y_{\perp}\right)=g^{2} \int_{-\infty}^{+\infty} d z_{*} d \omega_{*} \bar{\psi}\left(\omega_{*}, x_{\perp}\right)\left[\omega_{*}, \infty p_{1}\right]_{x} i \not p_{1}\left[-\infty p_{1}, z_{*}\right]_{y} \psi\left(z_{*}, y_{\perp}\right) \\
& \mathcal{X}_{5}\left(x_{\perp}, y_{\perp}\right)=-g^{2} \int_{-\infty}^{+\infty} d z_{*} d \omega_{*} \bar{\psi}\left(z_{*}, y_{\perp}\right)\left[z_{*},-\infty p_{1}\right]_{y} \gamma^{5} \not p_{1}\left[\infty p_{1}, \omega_{*}\right]_{x} \psi\left(\omega_{*}, x_{\perp}\right), \\
& \mathcal{X}_{5}^{\dagger}\left(x_{\perp}, y_{\perp}\right)=-g^{2} \int_{-\infty}^{+\infty} d z_{*} d \omega_{*} \bar{\psi}\left(\omega_{*}, x_{\perp}\right)\left[\omega_{*}, \infty p_{1}\right]_{x} \gamma^{5} \not p_{1}\left[-\infty p_{1}, z_{*}\right]_{y} \psi\left(z_{*}, y_{\perp}\right) .
\end{aligned}
$$

Operators (6.59)-(6.62) are not related, to the author's knowledge to any known Transverse Momentum Distributions (TMD).

We also define

$$
\begin{aligned}
& \mathcal{H}_{1}^{+}\left(x_{\perp}, y_{\perp}\right)=-g^{2} \int_{-\infty}^{+\infty} d z_{*} d \omega_{*} \bar{\psi}\left(\omega_{*}, y_{\perp}\right)\left[\omega_{*}, \infty p_{1}\right]_{y} i \not p_{1}\left[\infty p_{1}, z_{*}\right]_{x} \psi\left(z_{*}, x_{\perp}\right), \\
& \mathcal{H}_{5}^{+}\left(x_{\perp}, y_{\perp}\right)=-g^{2} \int_{-\infty}^{+\infty} d z_{*} d \omega_{*} \bar{\psi}\left(\omega_{*}, y_{\perp}\right)\left[\omega_{*}, \infty p_{1}\right]_{y} \gamma^{5} \not p_{1}\left[\infty p_{1}, z_{*}\right]_{x} \psi\left(z_{*}, x_{\perp}\right), \\
& \mathcal{H}_{1}^{-}\left(x_{\perp}, y_{\perp}\right)=-g^{2} \int_{-\infty}^{+\infty} d z_{*} d \omega_{*} \bar{\psi}\left(\omega_{*}, y_{\perp}\right)\left[\omega_{*},-\infty p_{1}\right]_{y} i p_{1}\left[-\infty p_{1}, z_{*}\right]_{x} \psi\left(z_{*}, x_{\perp}\right), \\
& \mathcal{H}_{5}^{-}\left(x_{\perp}, y_{\perp}\right)=-g^{2} \int_{-\infty}^{+\infty} d z_{*} d \omega_{*} \bar{\psi}\left(\omega_{*}, y_{\perp}\right)\left[\omega_{*},-\infty p_{1}\right]_{y} \gamma^{5} \not p_{1}\left[-\infty p_{1}, z_{*}\right]_{x} \psi\left(z_{*}, x_{\perp}\right),
\end{aligned}
$$

where the superscripts + and - remind that the semi-infinite Wilson lines point to $+\infty p_{1}$ and $-\infty p_{1}$ respectively. We will write subsequent results in terms of the above defined operators.

\subsubsection{Quark-to-gluon diagrams for $\tilde{Q}_{1 x}$ and $\tilde{Q}_{5 x}$}

Let us start with the calculation for operators $\tilde{Q}_{1 x}$ and $\tilde{Q}_{5 x}$. Diagram 12a is

$$
\begin{aligned}
\left\langle\operatorname{Tr}\left\{U_{y}^{\dagger} \tilde{Q}_{1 x}\right\}\right\rangle_{\text {Figure } 12 a} \\
=g^{2} \int_{-\infty}^{+\infty} d z_{*} \int_{-\infty}^{z_{*}} d z_{*}^{\prime} \operatorname{Tr}\left\{U_{y}^{\dagger}\right. \\
\left.\quad \times i g \int_{z_{*}}^{+\infty} d \frac{2}{s} x_{*} A_{*}^{q}\left(x_{*}, x_{\perp}\right)\left[\infty p_{1}, z_{*}\right]_{x} \operatorname{tr}\left\{\psi^{c l}\left(z_{*}, x_{\perp}\right) \bar{\psi}^{q}\left(z_{*}^{\prime}, x_{\perp}\right) i \not p_{1}\right\}\left[z_{*}^{\prime},-\infty p_{1}\right]_{x}\right\} \\
=-g^{4} \int_{-\infty}^{+\infty} d z_{*} \int_{-\infty}^{z_{*}} d z_{*}^{\prime} \int_{z_{*}}^{+\infty} d x_{*} \int_{z_{*}^{\prime}}^{x_{*}} d \omega_{*} \int_{0}^{+\infty} \frac{d \alpha}{s^{2} \alpha^{3}} \\
\quad \times \operatorname{Tr}\left\{U _ { y } ^ { \dagger } t ^ { a } [ \infty p _ { 1 } , z _ { * } ] _ { x } \operatorname { t r } \left\{\psi^{c l}\left(z_{*}, x_{\perp}\right)\right.\right. \\
\left.\left.\quad \times\left\langle x_{\perp}\left|e^{-i \frac{\hat{p}_{\perp}^{2}}{\alpha s} x_{*}} p_{\nu}^{\perp}\left[x_{*}, \omega_{*}\right]^{a b} \bar{\psi}^{c l}\left(\omega_{*}\right) t^{b} \gamma_{\perp}^{\nu}\left[\omega_{*}, z_{*}^{\prime}\right]\left(\alpha \not p_{1}+\not p_{\perp}\right) e^{i \frac{\hat{p}_{\perp}^{2}}{\alpha s} z_{*}^{\prime}}\right| x_{\perp}\right\rangle i \not p_{1}\right\}\right\}
\end{aligned}
$$

where we used propagator (6.55) and used the fact that the classical shock-wave field has line support only within the interval $\left[-\epsilon_{*}, \epsilon_{*}\right]$ (with $\epsilon_{*}>0$ ), and that, we are working in 


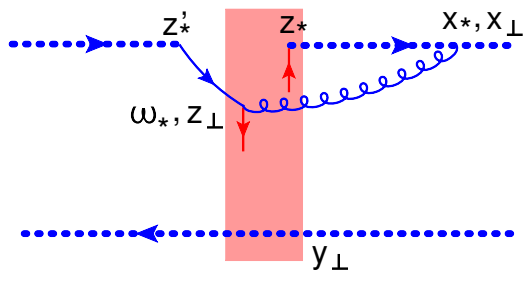

a)

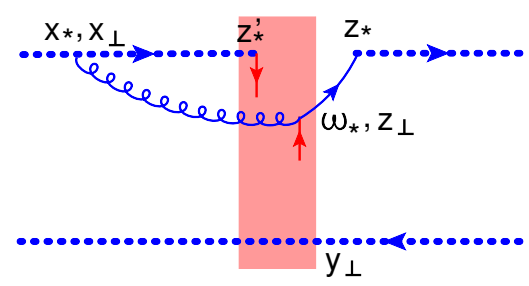

c)

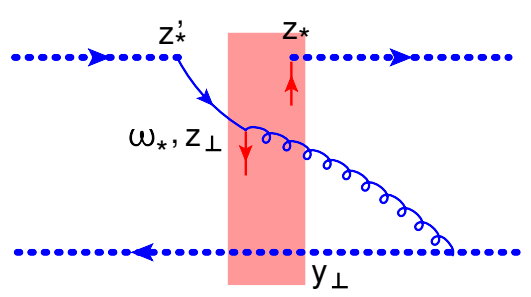

b)

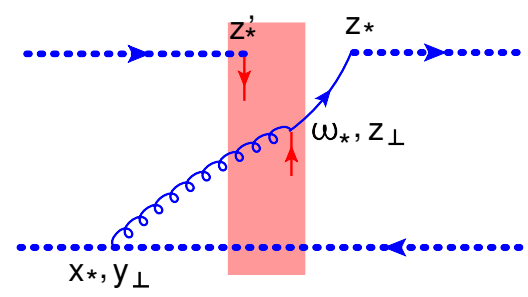

d)

Figure 12. Quark-to-gluon diagrams for $\hat{\tilde{Q}}_{1}\left(x_{\perp}\right)$ and $\hat{\tilde{Q}}_{5}\left(x_{\perp}\right)$ operators.

a gauge in which the gauge link made of pure gauge can be set to 1 . So, in the limit of $\epsilon_{*} \rightarrow 0$, the line support of the quantum fields, $x_{*}$ and $z_{*}^{\prime}$ (with the condition that $x_{*} \geq z_{*}^{\prime}$ ) extend up to 0 , and, on the other hand, the line support of the classical field can be extend to infinity, i.e. we extend the limit of integration of $\omega^{+}$to $]-\infty, \infty[$ and the gauge link becomes semi-infinite Wilson lines. Thus,

$$
\begin{aligned}
\left\langle\operatorname{Tr}\left\{U_{y}^{\dagger} \tilde{Q}_{1 x}\right\}\right\rangle_{\text {Figure 12a }}= & -g^{4} \int_{-\infty}^{+\infty} d z_{*} d \omega_{*} \int_{-\infty}^{0} d z_{*}^{\prime} \int_{0}^{+\infty} d x_{*} \int_{0}^{+\infty} \frac{d \alpha}{s^{2} \alpha^{3}} \int d^{2} z \\
& \times \operatorname{Tr}\left\{U _ { y } ^ { \dagger } t ^ { a } [ \infty p _ { 1 } , z _ { * } ] _ { x } \operatorname { t r } \left\{\psi^{c l}\left(z_{*}, x_{\perp}\right)\right.\right. \\
& \times\left\langle x_{\perp}\left|e^{-i \frac{\hat{p}_{\perp}^{2}}{\alpha s} x_{*}} p_{\nu}^{\perp}\right| z_{\perp}\right\rangle\left[\infty p_{1}, \omega_{*}\right]_{z}^{a b} \bar{\psi}^{c l}\left(\omega_{*}, z_{\perp}\right) t^{b} \gamma_{\perp}^{\nu} \\
& \left.\left.\times\left[\omega_{*},-\infty p_{1}\right]_{z}\left\langle z_{\perp}\left|\left(\alpha \not p_{1}+\not p_{\perp}\right) e^{i \frac{\hat{p}_{\perp}^{2}}{\alpha s} z_{*}^{\prime}}\right| x_{\perp}\right\rangle i \not p_{1}\right\}\right\}
\end{aligned}
$$

Next, we perform the integration over the light-cone coordinates $z^{\prime+}$ and $x^{+}$, and the Fourier transform

$$
\begin{aligned}
& \left\langle\operatorname{Tr}\left\{U_{y}^{\dagger} \tilde{Q}_{1 x}\right\}\right\rangle_{\text {Figure } 12 a}=\frac{g^{4}}{4 \pi^{2}} \int_{-\infty}^{+\infty} d z_{*} d \omega_{*} \int_{0}^{+\infty} \frac{d \alpha}{\alpha} \int d^{2} z \operatorname{Tr}\left\{U_{y}^{\dagger} t^{a}\left[\infty p_{1}, z_{*}\right]_{x}\right. \\
& \left.\quad \times \operatorname{tr}\left\{\psi^{c l}\left(z_{*}, x_{\perp}\right)\left[\infty p_{1}, \omega_{*}\right]_{z}^{a b} \bar{\psi}^{c l}\left(\omega_{*}, z_{\perp}\right) t^{b}\left[\omega_{*},-\infty p_{1}\right]_{z} \frac{(\not x-\not)_{\perp}}{(x-z)_{\perp}^{2}} \frac{(\not x-\not)_{\perp}}{(x-z)_{\perp}^{2}} i \not p_{1}\right\}\right\}
\end{aligned}
$$

After color and Dirac algebra we arrive at

$$
\left\langle\operatorname{Tr}\left\{U_{y}^{\dagger} \tilde{Q}_{1 x}\right\}\right\rangle_{\text {Figure } 12 a}=\frac{\alpha_{s}}{4 \pi^{2}} \int_{0}^{+\infty} \frac{d \alpha}{\alpha} \int \frac{d^{2} z}{(x-z)_{\perp}^{2}}\left[\frac{1}{N_{c}} \operatorname{Tr}\left\{U_{y}^{\dagger} \mathcal{X}_{1 x z}\right\}-\operatorname{Tr}\left\{U_{z} U_{y}^{\dagger}\right\} \mathcal{H}_{1 x z}^{+}\right]
$$


From eq. (6.69), substituting $i \not p_{1}$ with $\gamma^{5} \not p_{1}$ we get

$$
\left\langle\operatorname{Tr}\left\{U_{y}^{\dagger} \tilde{Q}_{5 x}\right\}\right\rangle_{\text {Figure } 12 a}=\frac{\alpha_{s}}{4 \pi^{2}} \int_{0}^{+\infty} \frac{d \alpha}{\alpha} \int \frac{d^{2} z}{(x-z)_{\perp}^{2}}\left[\frac{1}{N_{c}} \operatorname{Tr}\left\{U_{y}^{\dagger} \mathcal{X}_{5 x z}\right\}-\operatorname{Tr}\left\{U_{z} U_{y}^{\dagger}\right\} \mathcal{H}_{5 x z}^{+}\right]
$$

Let us turn to diagram in figure $12 \mathrm{~b}$ and perform similar steps as the ones we performed for $12 \mathrm{a}$

$$
\begin{aligned}
\left\langle\operatorname{Tr}\left\{U_{y}^{\dagger} \tilde{Q}_{1 x}\right\}\right\rangle_{\text {Figure } 12 b} \\
=-i g^{3} \int_{-\infty}^{+\infty} d z_{*} \int_{-\infty}^{z_{*}} d z_{*}^{\prime} \operatorname{Tr}\left\{U_{y}^{\dagger}\right. \\
\left.\quad \times \frac{2}{s} \int_{0}^{+\infty} d x_{*} A_{\bullet}\left(x_{*}, y_{\perp}\right)\left[\infty p_{1}, z_{*}\right]_{x} \operatorname{tr}\left\{i \not p_{1} \psi\left(z_{*}, x_{\perp}\right) \bar{\psi}\left(z_{*}^{\prime}, x_{\perp}\right)\right\}\left[z_{*}^{\prime},-\infty p_{1}\right]_{x}\right\} \\
=g^{4} \int_{-\infty}^{+\infty} d z_{*} \int_{-\infty}^{z_{*}} d z_{*}^{\prime} \int_{0}^{+\infty} d x_{*} \int_{z_{*}^{\prime}}^{+\infty} d \omega_{*} \int_{0}^{+\infty} \frac{d \alpha}{s^{2} \alpha^{3}} \operatorname{Tr}\left\{U_{y}^{\dagger} t^{a}\left[\infty p_{1}, z_{*}\right]_{x}\right. \\
\left.\quad \times \operatorname{tr}\left\{i \not p_{1} \psi\left(z_{*}, x_{\perp}\right)\left\langle y_{\perp}\left|e^{-i \frac{\hat{p}_{\perp}^{2}}{\alpha s} x_{*}} p_{\nu}^{\perp}\left[\infty p_{1}, \omega_{*}\right]^{a b} \bar{\psi}\left(\omega_{*}\right) t^{b} \gamma_{\perp}^{\nu}\left[\omega_{*},-\infty p_{1}\right] \not p_{\perp} e^{i \frac{\hat{p}_{\perp}^{2}}{\alpha s} z_{*}^{\prime}}\right| x_{\perp}\right\rangle\right\}\right\}
\end{aligned}
$$

Now we perform the integration over the light-cone variable $z^{\prime+}$ and $x^{+}$(taking into consideration the observations done for diagram 12a) and get

$$
\begin{aligned}
\left\langle\operatorname{Tr}\left\{U_{y}^{\dagger} \tilde{Q}_{1 x}\right\}\right\rangle_{\text {Figure } 12 b}= & -\frac{\alpha_{s}}{2 \pi^{2}} \int_{0}^{+\infty} \frac{d \alpha}{\alpha} \int_{-\infty}^{+\infty} d z_{*} d \omega_{*} \int d^{2} z \frac{(y-z)_{i}(x-z)_{j}}{(y-z)_{\perp}^{2}(z-x)_{\perp}^{2}} g^{2} \operatorname{Tr}\left\{U_{y}^{\dagger} t^{a}\right. \\
& \left.\times\left[\infty p_{1}, z_{*}\right]_{x} \operatorname{tr}\left\{\gamma^{i} \gamma^{j} i \not p_{1} \psi\left(z_{*}, x_{\perp}\right) \bar{\psi}\left(\omega_{*}, z_{\perp}\right)\right\}\left[\omega_{*}, \infty p_{1}\right]_{z} t^{a} U_{z}\right\}(6.73)
\end{aligned}
$$

Performing color and Dirac algebra we obtain

$$
\begin{aligned}
\left\langle\operatorname{Tr}\left\{U_{y}^{\dagger} \tilde{Q}_{1 x}\right\}\right\rangle_{\text {Figure } 12 b}= & -\frac{\alpha_{s}}{4 \pi^{2}} \int_{0}^{+\infty} \frac{d \alpha}{\alpha} \int d^{2} z \\
& \times\left\{\frac{(x-z, z-y)}{(y-z)_{\perp}^{2}(z-x)_{\perp}^{2}}\left(\operatorname{Tr}\left\{U_{z} U_{y}^{\dagger}\right\} \mathcal{H}_{1 x z}^{+}-\frac{1}{N_{c}} \operatorname{Tr}\left\{U_{y}^{\dagger} \mathcal{X}_{1 x z}\right\}\right)\right. \\
& \left.-\frac{(\vec{x}-\vec{z}) \times(\vec{y}-\vec{z})}{(y-z)_{\perp}^{2}(z-x)_{\perp}^{2}}\left(\operatorname{Tr}\left\{U_{z} U_{y}^{\dagger}\right\} \mathcal{H}_{5 x z}^{+}-\frac{1}{N_{c}} \operatorname{Tr}\left\{U_{y}^{\dagger} \mathcal{X}_{5 x z}\right\}\right)\right\}
\end{aligned}
$$

From (6.73), substituting $i \not p_{1}$ with $\gamma^{5} \not p_{1}$ we have

$$
\begin{aligned}
\left\langle\operatorname{Tr}\left\{U_{y}^{\dagger} \tilde{Q}_{5 x}\right\}\right\rangle_{\text {Figure 12b }}= & -\frac{\alpha_{s}}{4 \pi^{2}} \int_{0}^{+\infty} \frac{d \alpha}{\alpha} \int d^{2} z \\
& \times\left\{\frac{(x-z, z-y)}{(y-z)_{\perp}^{2}(z-x)_{\perp}^{2}}\left(\operatorname{Tr}\left\{U_{z} U_{y}^{\dagger}\right\} \mathcal{H}_{5 x z}^{+}-\frac{1}{N_{c}} \operatorname{Tr}\left\{U_{y}^{\dagger} \mathcal{X}_{5 x z}\right\}\right)\right. \\
& \left.+\frac{(\vec{x}-\vec{z}) \times(\vec{y}-\vec{z})}{(y-z)_{\perp}^{2}(z-x)_{\perp}^{2}}\left(\operatorname{Tr}\left\{U_{z} U_{y}^{\dagger}\right\} \mathcal{H}_{1 x z}^{+}-\frac{1}{N_{c}} \operatorname{Tr}\left\{U_{y}^{\dagger} \mathcal{X}_{1 x z}\right\}\right)\right\} .
\end{aligned}
$$


The calculation for diagrams $12 \mathrm{c}$ and $12 \mathrm{~d}$ is the same as the previous two ones, so we may write

$$
\begin{aligned}
& \left\langle\operatorname{Tr}\left\{U_{y}^{\dagger} \tilde{Q}_{1 x}\right\}\right\rangle_{\text {Figure } 12 c}=i g^{3} \int_{-\infty}^{+\infty} d z_{*} \int_{-\infty}^{z_{*}} d z_{*}^{\prime} \int_{-\infty}^{z_{*}^{\prime}} d \frac{2}{s} x_{*} \operatorname{Tr}\left\{U_{y}^{\dagger}\left[\infty p_{1}, z_{*}\right]_{x}\right. \\
& \left.\times \operatorname{tr}\left\{\psi\left(z_{*}, x_{\perp}\right) \bar{\psi}\left(z_{*}^{\prime}, x_{\perp}\right) i \not p_{1}\right\}\left[z_{*}^{\prime},-\infty p_{1}\right]_{x} A_{\bullet}\left(x_{*}, x_{\perp}\right)\right\} \\
& =-g^{4} \int_{-\infty}^{+\infty} d z_{*}^{\prime} \int_{z_{*}^{\prime}}^{+\infty} d z_{*} \int_{-\infty}^{z_{*}^{\prime}} d \frac{2}{s} x_{*} \int_{x_{*}}^{z_{*}} d \frac{2}{s} \omega_{*} \int_{0}^{+\infty} \frac{d \alpha}{4 \alpha^{3}} \int d^{2} z \\
& \times \operatorname{Tr}\left\{U _ { y } ^ { \dagger } \operatorname { t r } \left\{i \not p_{1}\left\langle x_{\perp}\left|e^{-i \frac{\hat{p}_{\perp}^{2}}{\alpha s} z_{*}}\left(\alpha \not p_{1}+\not p_{\perp}\right)\right| z_{\perp}\right\rangle\left[z_{*}, \omega_{*}\right] \gamma_{\perp}^{\nu} t^{b} \psi\left(\omega_{*}\right)\left[\omega_{*}, x_{*}\right]^{b a}\right.\right. \\
& \left.\left.\times\left\langle z_{\perp}\left|p_{\nu}^{\perp} e^{i \frac{\hat{p}_{\perp}^{2}}{\alpha s} x_{*}}\right| x_{\perp}\right\rangle \bar{\psi}\left(z_{*}^{\prime}, x_{\perp}\right)\right\}\left[z_{*}^{\prime},-\infty p_{1}\right]_{x} t^{a}\right\} \\
& =-\frac{\alpha_{s}}{4 \pi^{2}} \int_{0}^{+\infty} \frac{d \alpha}{\alpha} \int \frac{d^{2} z}{(x-z)_{\perp}^{2}}\left[\operatorname{Tr}\left\{U_{y}^{\dagger} U_{z}\right\} \mathcal{H}_{1 z x}^{-}-\frac{1}{N_{c}} \operatorname{Tr}\left\{U_{y}^{\dagger} \mathcal{X}_{1 z x}\right\}\right]
\end{aligned}
$$

For $\tilde{Q}_{5 x}$ we have

$$
\left\langle\operatorname{Tr}\left\{U_{y}^{\dagger} \tilde{Q}_{5 x}\right\}\right\rangle_{\text {Figure } 12 c}=-\frac{\alpha_{s}}{4 \pi^{2}} \int_{0}^{+\infty} \frac{d \alpha}{\alpha} \int \frac{d^{2} z}{(x-z)_{\perp}^{2}}\left[\operatorname{Tr}\left\{U_{y}^{\dagger} U_{z}\right\} \mathcal{H}_{5 z x}^{-}-\frac{1}{N_{c}} \operatorname{Tr}\left\{U_{y}^{\dagger} \mathcal{X}_{5 z x}\right\}\right]
$$

Finally, diagram $12 \mathrm{~d}$ is

$$
\begin{aligned}
\left\langle\operatorname{Tr}\left\{U_{y}^{\dagger} \tilde{Q}_{1 x}\right\}\right\rangle_{\text {Figure 12d }}= & -\frac{\alpha_{s}}{4 \pi^{2}} \int_{0}^{+\infty} \frac{d \alpha}{\alpha} \int d^{2} z \\
& \times\left\{\frac{(x-z, z-y)}{(y-z)_{\perp}^{2}(z-x)_{\perp}^{2}}\left(\operatorname{Tr}\left\{U_{z} U_{y}^{\dagger}\right\} \mathcal{H}_{1 z x}^{-}-\frac{1}{N_{c}} \operatorname{Tr}\left\{U_{y}^{\dagger} \mathcal{X}_{1 z x}\right\}\right)\right. \\
& \left.+\frac{(\vec{x}-\vec{z}) \times(\vec{y}-\vec{z})}{(y-z)_{\perp}^{2}(z-x)_{\perp}^{2}}\left(\operatorname{Tr}\left\{U_{z} U_{y}^{\dagger}\right\} \mathcal{H}_{5 z x}^{-}-\frac{1}{N_{c}} \operatorname{Tr}\left\{U_{y}^{\dagger} \mathcal{X}_{5 z x}\right\}\right)\right\}
\end{aligned}
$$

For $\tilde{Q}_{5 x}$ we have

$$
\begin{aligned}
\left\langle\operatorname{Tr}\left\{U_{y}^{\dagger} \tilde{Q}_{5 x}\right\}\right\rangle_{\text {Figure 12d }}= & -\frac{\alpha_{s}}{4 \pi^{2}} \int_{0}^{+\infty} \frac{d \alpha}{\alpha} \int d^{2} z \\
& \times\left\{\frac{(x-z, z-y)}{(y-z)_{\perp}^{2}(z-x)_{\perp}^{2}}\left(\operatorname{Tr}\left\{U_{z} U_{y}^{\dagger}\right\} \mathcal{H}_{5 z x}^{-}-\frac{1}{N_{c}} \operatorname{Tr}\left\{U_{y}^{\dagger} \mathcal{X}_{5 z x}\right\}\right)\right. \\
& \left.-\frac{(\vec{x}-\vec{z}) \times(\vec{y}-\vec{z})}{(y-z)_{\perp}^{2}(z-x)_{\perp}^{2}}\left(\operatorname{Tr}\left\{U_{z} U_{y}^{\dagger}\right\} \mathcal{H}_{1 z x}^{-}-\frac{1}{N_{c}} \operatorname{Tr}\left\{U_{y}^{\dagger} \mathcal{X}_{1 z x}\right\}\right)\right\}
\end{aligned}
$$


We now may sum up diagrams in figure 12 . Starting with $\tilde{Q}_{1 x}$, we sum eqs. (6.70), (6.74), (6.76), and (6.78) we have

$$
\begin{aligned}
\left\langle\operatorname{Tr}\left\{U_{y}^{\dagger} \tilde{Q}_{1 x}\right\}\right\rangle_{\text {Figure } 12} \\
=-\frac{\alpha_{s}}{4 \pi^{2}} \int_{0}^{+\infty} \frac{d \alpha}{\alpha} \int d^{2} z\left\{\frac{1}{(x-z)_{\perp}^{2}}\left[\operatorname{Tr}\left\{U_{z} U_{y}^{\dagger}\right\}\left(\mathcal{H}_{1 x z}^{+}+\mathcal{H}_{1 z x}^{-}\right)-\frac{1}{N_{c}} \operatorname{Tr}\left\{U_{y}^{\dagger}\left(\mathcal{X}_{1 x z}+\mathcal{X}_{1 z x}\right)\right\}\right]\right. \\
\quad+\frac{(x-z, z-y)}{(y-z)_{\perp}^{2}(z-x)_{\perp}^{2}}\left[\operatorname{Tr}\left\{U_{z} U_{y}^{\dagger}\right\}\left(\mathcal{H}_{1 x z}^{+}+\mathcal{H}_{1 z x}^{-}\right)-\frac{1}{N_{c}} \operatorname{Tr}\left\{U_{y}^{\dagger}\left(\mathcal{X}_{1 x z}+\mathcal{X}_{1 z x}\right)\right\}\right] \\
\left.\quad+\frac{(\vec{x}-\vec{z}) \times(\vec{y}-\vec{z})}{(y-z)_{\perp}^{2}(z-x)_{\perp}^{2}}\left[\operatorname{Tr}\left\{U_{z} U_{y}^{\dagger}\right\}\left(\mathcal{H}_{5 z x}^{-}-\mathcal{H}_{5 x z}^{+}\right)+\frac{1}{N_{c}} \operatorname{Tr}\left\{U_{y}^{\dagger}\left(\mathcal{X}_{5 x z}-\mathcal{X}_{5 z x}\right)\right\}\right]\right\} .
\end{aligned}
$$

The sum of diagrams in figure 12 with $\tilde{Q}_{5 x}$ is obtained summing eqs. (6.71), (6.75), (6.77), and (6.79). So, we have

$$
\begin{aligned}
& \left\langle\operatorname{Tr}\left\{U_{y}^{\dagger} \tilde{Q}_{5 x}\right\}\right\rangle_{\text {Figure } 12} \\
& =-\frac{\alpha_{s}}{4 \pi^{2}} \int_{0}^{+\infty} \frac{d \alpha}{\alpha} \int d^{2} z\left\{\frac{1}{(x-z)_{\perp}^{2}}\left[\operatorname{Tr}\left\{U_{z} U_{y}^{\dagger}\right\}\left(\mathcal{H}_{5 x z}^{+}+\mathcal{H}_{5 z x}^{-}\right)-\frac{1}{N_{c}} \operatorname{Tr}\left\{U_{y}^{\dagger}\left(\mathcal{X}_{5 x z}+\mathcal{X}_{5 z x}\right)\right\}\right]\right. \\
& \left.\quad+\frac{(x-z, z-y)}{(y-z)_{\perp}^{2}(z-x)_{\perp}^{2}}\left[\operatorname{Tr}\left\{U_{z} U_{y}^{\dagger}\right\}\left(\mathcal{H}_{5 x z}^{+}+\mathcal{H}_{5 z x}^{-}\right)-\frac{1}{N_{c}} \operatorname{Tr}\left\{U_{y}^{\dagger}\left(\mathcal{X}_{5 x z}+\mathcal{X}_{5 z x}\right)\right\}\right)\right] \\
& \left.\quad+\frac{(\vec{x}-\vec{z}) \times(\vec{y}-\vec{z})}{(y-z)_{\perp}^{2}(z-x)_{\perp}^{2}}\left[\operatorname{Tr}\left\{U_{z} U_{y}^{\dagger}\right\}\left(\mathcal{H}_{1 x z}^{+}-\mathcal{H}_{1 z x}^{-}\right)+\frac{1}{N_{c}} \operatorname{Tr}\left\{U_{y}^{\dagger}\left(\mathcal{X}_{1 z x}-\mathcal{X}_{1 x z}\right)\right\}\right]\right\} .
\end{aligned}
$$

\subsubsection{Quark-to-gluon diagrams for $\hat{Q}_{1 x}$ and $\hat{Q}_{5 x}$}

Now we turn our attention to the diagrams with the quark-to-gluon propagator for operators $\operatorname{Tr}\left\{\hat{U}_{x} \hat{U}_{y}^{\dagger}\right\} \hat{Q}_{1 x}$ and $\operatorname{Tr}\left\{\hat{U}_{x} \hat{U}_{y}^{\dagger}\right\} \hat{Q}_{5 x}$ given in figure 13. Note that, these type of diagrams will, after one loop, make the dipole operator $\operatorname{Tr}\left\{\hat{U}_{x} \hat{U}_{y}^{\dagger}\right\}$ talk with operator $\hat{Q}_{1 x}$ (or $\hat{Q}_{5 x}$ ).

Let us start with the calculation of the first diagram, 13a. The procedure is similar to the one adopted in the previous section

$$
\begin{aligned}
\left\langle\operatorname{Tr}\left\{U_{x} U_{y}^{\dagger}\right\} Q_{1 x}\right\rangle_{\text {Figure 13a }}= & i g^{3} \frac{2}{s} \int_{-\infty}^{+\infty} d z_{*} \int_{-\infty}^{z_{*}} d z_{*}^{\prime} \int_{0}^{+\infty} d x_{*} \operatorname{Tr}\left\{t^{a} U_{x} U_{y}^{\dagger}\right\} \\
& \times \operatorname{Tr}\left\{\left[z_{*}^{\prime}, z_{*}\right]_{x} \operatorname{tr}\left\{i \not p_{1} \psi^{c l}\left(z_{*}, x_{\perp}\right)\left\langle A_{\bullet}^{q}\left(x_{*}, x_{\perp}\right) \bar{\psi}^{q}\left(z_{*}^{\prime}, x_{\perp}\right)\right\rangle\right\}\right\} .
\end{aligned}
$$

Using propagator (6.55) we have

$$
\begin{aligned}
\left\langle\operatorname{Tr}\left\{U_{x} U_{y}^{\dagger}\right\} Q_{1 x}\right\rangle_{\text {Figure 13a }}= & -g^{4} \int_{-\infty}^{+\infty} d z_{*} \int_{-\infty}^{z_{*}} d z_{*}^{\prime} \int_{0}^{+\infty} d x_{*} \int_{z_{*}^{\prime}}^{x_{*}} d \omega_{*} \int_{0}^{+\infty} \frac{d \alpha}{s^{2} \alpha^{3}} \operatorname{Tr}\left\{t^{a} U_{x} U_{y}^{\dagger}\right\} \\
& \times \int d^{2} z \operatorname{Tr}\left\{[ - \infty p _ { 1 } , z _ { * } ] _ { x } \operatorname { t r } \left\{i \not p_{1} \psi\left(z_{*}, x_{\perp}\right)\left\langle x_{\perp}\left|e^{-i \frac{\hat{p}_{\perp}^{2}}{\alpha s} x_{*}} p_{\nu}^{\perp}\right| z_{\perp}\right\rangle(6.83)\right.\right. \\
& \left.\left.\times\left[\infty p_{1}, \omega_{*}\right]_{z}^{a b} \bar{\psi}\left(\omega_{*}, z_{\perp}\right) t^{b} \gamma_{\perp}^{\nu}\left[\omega_{*},-\infty p_{1}\right]_{z}\left\langle z_{\perp}\left|\not p_{\perp} e^{i \frac{\hat{p}_{\perp}^{2}}{\alpha s} z_{*}^{\prime}}\right| x_{\perp}\right\rangle\right\}\right\} .
\end{aligned}
$$




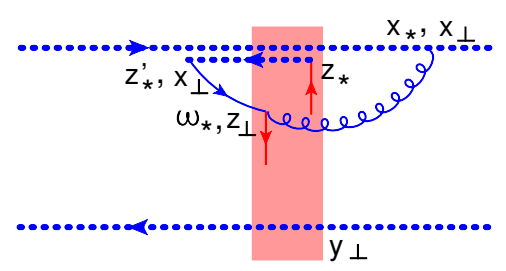

a)

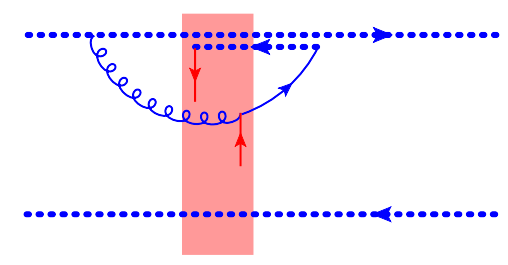

c)

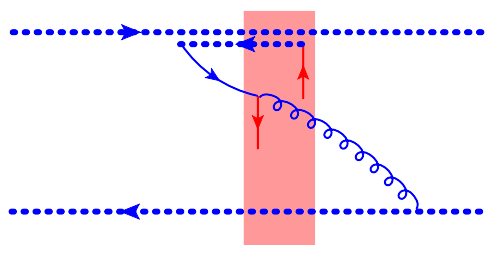

b)

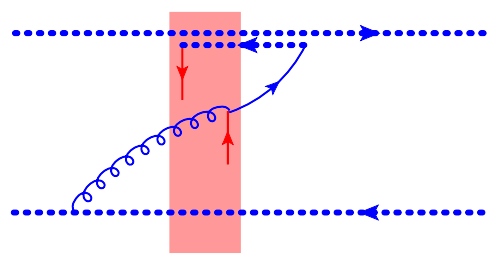

d)

Figure 13. Quark-to-gluon diagrams for $\hat{Q}_{1}\left(x_{\perp}\right)$ and $\hat{Q}_{5}\left(x_{\perp}\right)$ operators.

Next step is again to recognize the range of the quantum and classical fields as we did for diagram in figure 12 . So, we have

$$
\begin{aligned}
\left\langle\operatorname{Tr}\left\{U_{x} U_{y}^{\dagger}\right\} Q_{1 x}\right\rangle_{\text {Figure } 13 a} \\
=-\frac{\alpha_{s}}{2 \pi^{2}} \int_{0}^{+\infty} \frac{d \alpha}{\alpha} g^{2} \int_{-\infty}^{+\infty} d z_{*} d \omega_{*} \int \frac{d^{2} z}{(x-z)_{\perp}^{2}} \\
\quad \times \operatorname{Tr}\left\{t^{a} U_{x} U_{y}^{\dagger}\right\} \operatorname{Tr}\left\{\left[-\infty p_{1}, z_{*}\right]_{x} \operatorname{tr}\left\{i \not p_{1} \psi\left(z_{*}, x_{\perp}\right) \bar{\psi}\left(\omega_{*}, z_{\perp}\right)\right\}\left[\omega_{*}, \infty p_{1}\right]_{z} t^{a} U_{z}\right\} \\
=-\frac{\alpha_{s}}{2 \pi^{2}} \int_{0}^{+\infty} \frac{d \alpha}{\alpha} g^{2} \int_{-\infty}^{+\infty} d z_{*} d \omega_{*} \int \frac{d^{2} z}{(x-z)_{\perp}^{2}} \\
\quad \times\left[\frac{1}{2} \operatorname{Tr}\left\{U_{x} U_{y}^{\dagger} U_{z}\left[-\infty p_{1}, z_{*}\right]_{x} \operatorname{tr}\left\{i \not p_{1} \psi\left(z_{*}, x_{\perp}\right) \bar{\psi}\left(\omega_{*}, z_{\perp}\right)\right\}\left[\omega_{*}, \infty p_{1}\right]_{z}\right\}\right. \\
\\
\left.-\frac{1}{2 N_{c}} \operatorname{Tr}\left\{U_{x} U_{y}^{\dagger}\right\} \operatorname{Tr}\left\{\left[-\infty p_{1}, z_{*}\right]_{x} \operatorname{tr}\left\{i \not p_{1} \psi\left(z_{*}, x_{\perp}\right) \bar{\psi}\left(\omega_{*}, z_{\perp}\right)\right\}\left[\omega_{*},-\infty p_{1}\right]_{z}\right\}\right] .
\end{aligned}
$$

Using definition of operators (6.59)-(6.62) and (6.63)-(6.66) we arrive at

$$
\begin{aligned}
\left\langle\operatorname{Tr}\left\{U_{x} U_{y}^{\dagger}\right\} Q_{1 x}\right\rangle_{\text {Figure 13a }}= & \frac{\alpha_{s}}{4 \pi^{2}} \int_{0}^{+\infty} \frac{d \alpha}{\alpha} \int \frac{d^{2} z}{(x-z)_{\perp}^{2}} \\
& \times\left[\operatorname{Tr}\left\{U_{x} U_{y}^{\dagger} U_{z} \mathcal{X}_{1 z x}^{\dagger}\right\}+\frac{1}{N_{c}} \operatorname{Tr}\left\{U_{x} U_{y}^{\dagger}\right\} \mathcal{H}_{1 x z}^{-}\right]
\end{aligned}
$$

Substituting $i \not p_{1}$ with $\gamma^{5} \not p_{1}$ we get

$$
\begin{aligned}
\left\langle\operatorname{Tr}\left\{U_{x} U_{y}^{\dagger}\right\} Q_{5 x}\right\rangle_{\text {Figure 13a }}= & -\frac{\alpha_{s}}{4 \pi^{2}} \int_{0}^{+\infty} \frac{d \alpha}{\alpha} \int \frac{d^{2} z}{(x-z)_{\perp}^{2}} \\
& \times\left[\operatorname{Tr}\left\{U_{x} U_{y}^{\dagger} U_{z} \mathcal{X}_{5 z x}^{\dagger}\right\}-\frac{1}{N_{c}} \operatorname{Tr}\left\{U_{x} U_{y}^{\dagger}\right\} \mathcal{H}_{5 x z}^{-}\right]
\end{aligned}
$$


Similarly, for diagram $13 \mathrm{~b}$ we have

$$
\begin{aligned}
& \left\langle\operatorname{Tr}\left\{U_{x} U_{y}^{\dagger}\right\} Q_{1 x}\right\rangle_{\text {Figure } 13 b} \\
& =-i g^{3} \frac{2}{s} \int_{-\infty}^{+\infty} d z_{*} \int_{-\infty}^{z_{*}} d z_{*}^{\prime} \int_{0}^{+\infty} d x_{*} \\
& \quad \times \operatorname{Tr}\left\{U_{x} U_{y}^{\dagger} t^{a}\right\} \operatorname{Tr}\left\{\left[z_{*}^{\prime}, z_{*}\right]_{x} \operatorname{tr}\left\{i \not p_{1} \psi^{c l}\left(z_{*}, x_{\perp}\right)\left\langle A_{*}^{q, a}\left(x_{*}, y_{\perp}\right) \bar{\psi}^{q}\left(z_{*}^{\prime}, x_{\perp}\right)\right\rangle\right\}\right\} \\
& =g^{4} \int_{-\infty}^{+\infty} d z_{*} \int_{-\infty}^{z_{*}} d z_{*}^{\prime} \int_{0}^{+\infty} d x_{*} \int_{z_{*}^{\prime}}^{x_{*}} d \omega_{*} \int_{0}^{+\infty} \frac{d \alpha}{s^{2} \alpha^{3}} \operatorname{Tr}\left\{U_{x} U_{y}^{\dagger} t^{a}\right\} \int d^{2} z \\
& \quad \times \operatorname{Tr}\left\{[ z _ { * } ^ { \prime } , z _ { * } ] _ { x } \operatorname { t r } \left\{i \not p_{1} \psi\left(z_{*}, x_{\perp}\right)\left\langle y_{\perp}\left|e^{-i \frac{\hat{p}_{\perp}^{2}}{\alpha s} x_{*}} p_{\perp}^{\nu}\right| z_{\perp}\right\rangle\left[x_{*}, \omega_{*}\right]_{z}^{a b} \bar{\psi}\left(\omega_{*}, z_{\perp}\right)\right.\right. \\
& \left.\left.\quad \times t^{b} \gamma_{\nu}^{\perp}\left[\omega_{*}, z_{*}^{\prime}\right]_{z}\left\langle z_{\perp}\left|\not p_{\perp} e^{i \frac{\hat{p}_{\perp}^{2}}{\alpha s} z_{*}^{\prime}}\right| x_{\perp}\right\rangle\right\}\right\} .
\end{aligned}
$$

After Fourier transform and integration over the light-cone coordinates we have

$$
\begin{aligned}
\left\langle\operatorname{Tr}\left\{U_{x} U_{y}^{\dagger}\right\} Q_{1 x}\right\rangle_{\text {Figure 13b }}= & -\frac{\alpha_{s}}{2 \pi^{2}} g^{2} \int_{-\infty}^{+\infty} d z_{*} d \omega_{*} \int_{0}^{+\infty} \frac{d \alpha}{\alpha} \operatorname{Tr}\left\{U_{x} U_{y}^{\dagger} t^{a}\right\} \int d^{2} z \\
& \times \operatorname{Tr}\left\{[ - \infty p _ { 1 } , z _ { * } ] _ { x } \operatorname { t r } \left\{i \not p_{1} \psi\left(z_{*}, x_{\perp}\right) \frac{(y-z)_{\perp}^{\nu}}{(y-z)_{\perp}^{2}}\left[\infty p_{1}, \omega_{*}\right]_{z}^{a b} \bar{\psi}\left(\omega_{*}, z_{\perp}\right)\right.\right. \\
& \left.\left.\times t^{b} \gamma_{\nu}^{\perp}\left[\omega_{*},-\infty p_{1}\right]_{z} \frac{(\not p-\not z)}{(x-z)_{\perp}^{2}}\right\}\right\} .
\end{aligned}
$$

Performing color and Dirac algebra we obtain

$$
\begin{aligned}
\left\langle\operatorname{Tr}\left\{U_{x} U_{y}^{\dagger}\right\} Q_{1 x}\right\rangle_{\text {Figure 13b }}= & \frac{\alpha_{s}}{4 \pi^{2}} \int_{0}^{+\infty} \frac{d \alpha}{\alpha} \int d^{2} z \\
& \times\left\{\frac{(x-z, z-y)_{\perp}}{(y-z)_{\perp}^{2}(x-z)_{\perp}^{2}}\left(\operatorname{Tr}\left\{U_{x} U_{y}^{\dagger} U_{z} \mathcal{X}_{1 z x}^{\dagger}\right\}+\frac{1}{N_{c}} \operatorname{Tr}\left\{U_{x} U_{y}^{\dagger}\right\} \mathcal{H}_{1 x z}^{-}\right)\right. \\
& \left.+\frac{(\vec{x}-\vec{z}) \times(\vec{y}-\vec{z})}{(y-z)_{\perp}^{2}(x-z)_{\perp}^{2}}\left(\operatorname{Tr}\left\{U_{x} U_{y}^{\dagger} U_{z} \mathcal{X}_{5 z x}^{\dagger}\right\}-\frac{1}{N_{c}} \operatorname{Tr}\left\{U_{x} U_{y}^{\dagger}\right\} \mathcal{H}_{5 x z}^{-}\right)\right\} .
\end{aligned}
$$

For $Q_{5}\left(x_{\perp}\right)$ we have

$$
\begin{aligned}
\left\langle\operatorname{Tr}\left\{U_{x} U_{y}^{\dagger}\right\} Q_{5 x}\right\rangle_{\text {Figure 13b }}= & -\frac{\alpha_{s}}{4 \pi^{2}} \int_{0}^{+\infty} \frac{d \alpha}{\alpha} \int d^{2} z \\
& \times\left\{\frac{(x-z, z-y)_{\perp}}{(y-z)_{\perp}^{2}(x-z)_{\perp}^{2}}\left(\operatorname{Tr}\left\{U_{x} U_{y}^{\dagger} U_{z} \mathcal{X}_{5 z x}^{\dagger}\right\}-\frac{1}{N_{c}} \operatorname{Tr}\left\{U_{x} U_{y}^{\dagger}\right\} \mathcal{H}_{5 x z}^{-}\right)\right. \\
& \left.-\frac{(\vec{x}-\vec{z}) \times(\vec{y}-\vec{z})}{(y-z)_{\perp}^{2}(x-z)_{\perp}^{2}}\left(\operatorname{Tr}\left\{U_{x} U_{y}^{\dagger} U_{z} \mathcal{X}_{1 z x}^{\dagger}\right\}+\frac{1}{N_{c}} \operatorname{Tr}\left\{U_{x} U_{y}^{\dagger}\right\} \mathcal{H}_{1 x z}^{-}\right)\right\}
\end{aligned}
$$


Diagram in figure $13 \mathrm{c}$ is

$$
\begin{aligned}
\left\langle\operatorname{Tr}\left\{U_{x} U_{z}^{\dagger}\right\} Q_{1 x}\right\rangle_{\text {Figure } 13 c} & =g^{4} \int_{-\infty}^{+\infty} d z_{*}^{\prime} d \omega_{*} \int_{z_{*}^{\prime}}^{+\infty} d z_{*} \int_{\omega_{*}}^{0} d x_{*} \int_{0}^{+\infty} \frac{d \alpha}{\alpha^{3} s^{2}} \operatorname{Tr}\left\{U_{x} t^{a} U_{y}^{\dagger}\right\} \\
& \times \int d^{2} z \operatorname{Tr}\left\{\operatorname { t r } \left\{i \not p_{1}\left\langle x_{\perp}\left|e^{-i \frac{\hat{p}_{\perp}^{\perp}}{\alpha s} z_{*}} \not p_{\perp}\right| z_{\perp}\right\rangle\left[\infty p_{1}, \omega_{*}\right]_{z} \gamma_{\perp}^{\nu} t^{b} \psi\left(\omega_{*}, z_{\perp}\right)\left[\omega_{*}, x_{*}\right]_{z}^{b a}\right.\right. \\
& \left.\left.\times\left\langle z_{\perp}\left|p_{\perp}^{\nu} e^{i \frac{\hat{p}_{\perp}^{2}}{\alpha s} x_{*}}\right| x_{\perp}\right\rangle \bar{\psi}\left(z_{*}^{\prime}, x_{\perp}\right)\left[z_{*}^{\prime}, \infty p_{1}\right]_{x}\right\}\right\} \\
= & -\frac{\alpha_{s}}{2 \pi^{2}} \int_{0}^{+\infty} \frac{d \alpha}{\alpha} g^{2} \int_{-\infty}^{+\infty} d z_{*}^{\prime} d \omega_{*} \int \frac{d^{2} z}{(x-z)_{\perp}^{2}} \operatorname{Tr}\left\{U_{y}^{\dagger} U_{x} t^{a}\right\} \\
& \times \operatorname{Tr}\left\{t^{a}\left[-\infty p_{1}, \omega_{*}\right]_{z} \operatorname{tr}\left\{i \not p_{1} \psi\left(\omega_{*}, z_{\perp}\right) \bar{\psi}\left(z_{*}^{\prime}, x_{\perp}\right)\right\}\left[z_{*}^{\prime}, \infty p_{1}\right]_{x} U_{z}\right\} \\
= & \frac{\alpha_{s}}{4 \pi^{2}} \int_{0}^{+\infty} \frac{d \alpha}{\alpha} \int \frac{d^{2} z}{(x-z)_{\perp}^{2}}\left[\operatorname{Tr}\left\{U_{z} U_{y}^{\dagger} U_{x} \mathcal{X}_{1 x z}^{\dagger}\right\}+\frac{1}{N_{c}} \operatorname{Tr}\left\{U_{y}^{\dagger} U_{x}\right\} \mathcal{H}_{1 z x}^{+}\right] .
\end{aligned}
$$

For operator $Q_{5 x}$ we have

$$
\begin{aligned}
\left\langle\operatorname{Tr}\left\{U_{x} U_{z}^{\dagger}\right\} Q_{5 x}\right\rangle_{\text {Figure 13c }}= & -\frac{\alpha_{s}}{4 \pi^{2}} \int_{0}^{+\infty} \frac{d \alpha}{\alpha} \int \frac{d^{2} z}{(x-z)_{\perp}^{2}} \\
& \times\left[\operatorname{Tr}\left\{U_{z} U_{y}^{\dagger} U_{x} \mathcal{X}_{5 x z}^{\dagger}\right\}-\frac{1}{N_{c}} \operatorname{Tr}\left\{U_{y}^{\dagger} U_{x}\right\} \mathcal{H}_{5 z x}^{+}\right] .
\end{aligned}
$$

Last diagram of this set of diagrams is 13c. The steps we have to perform to calculate this diagram are again the same as the ones performed above. We have

$$
\begin{aligned}
& \left\langle\operatorname{Tr}\left\{U_{x} U_{y}^{\dagger}\right\} Q_{1 x}\right\rangle_{\text {Figure } 13 d} \\
& =g^{4} \int_{-\infty}^{+\infty} d z_{*}^{\prime} \int_{-\infty}^{z_{*}} d \omega_{*} \int_{z_{*}^{\prime}}^{+\infty} d z_{*} \int_{-\infty}^{0} d x_{*} \int_{0}^{+\infty} \frac{d \alpha}{\alpha^{3} s^{2}} \operatorname{Tr}\left\{U_{x} t^{a} U_{y}^{\dagger}\right\} \\
& \quad \times \int d^{2} z \operatorname{Tr}\left\{[ z _ { * } ^ { \prime } , z _ { * } ] _ { x } \operatorname { t r } \left\{i \not \phi_{1}\left\langle x_{\perp}\left|e^{-i \frac{\hat{p}_{\perp}^{2}}{\alpha s} z_{*}} \not p_{\perp}\right| z_{\perp}\right\rangle\left[z_{*}, \omega_{*}\right]_{z} \gamma_{\perp}^{\nu} t^{b} \psi\left(\omega_{*}, z_{\perp}\right)\left[\omega_{*}, x_{*}\right]_{z}^{b a}\right.\right. \\
& \left.\left.\quad \times\left\langle z_{\perp}\left|p_{\nu}^{\perp} e^{i \frac{\hat{p}_{\perp}^{2}}{\alpha s} x_{*}}\right| y_{\perp}\right\rangle \bar{\psi}\left(z_{*}^{\prime}, x_{\perp}\right)\right\}\right\} .
\end{aligned}
$$

Fourier transform and integration over the longitudinal coordinates give

$$
\begin{aligned}
& \left\langle\operatorname{Tr}\left\{U_{x} U_{y}^{\dagger}\right\} Q_{1 x}\right\rangle_{\text {Figure } 13 d} \\
& =\frac{\alpha_{s}}{2 \pi^{2}} \int_{0}^{+\infty} \frac{d \alpha}{\alpha} g^{2} \int_{-\infty}^{+\infty} d z_{*}^{\prime} d \omega_{*} \int d^{2} z \frac{(x-z)_{i}(z-y)_{j}}{(x-z)_{\perp}^{2}(z-y)_{\perp}^{2}} \operatorname{Tr}\left\{U_{y}^{\dagger} U_{x} t^{a}\right\} \\
& \quad \times \operatorname{Tr}\left\{t^{a}\left[-\infty p_{1}, \omega_{*}\right]_{z} \operatorname{tr}\left\{i \not p_{1} \gamma^{i} \gamma^{j} \psi\left(\omega_{*}, z_{\perp}\right) \bar{\psi}\left(z_{*}^{\prime}, x_{\perp}\right)\right\}\left[z_{*}^{\prime}, \infty p_{1}\right]_{x} U_{z}\right\} .
\end{aligned}
$$

Color and Dirac algebra give

$$
\begin{aligned}
& \left\langle\operatorname{Tr}\left\{U_{x} U_{y}^{\dagger}\right\} Q_{1 x}\right\rangle_{\text {Figure } 13 d} \\
& =\frac{\alpha_{s}}{4 \pi^{2}} \int_{0}^{+\infty} \frac{d \alpha}{\alpha} \int d^{2} z\left\{\frac{(x-z, z-y)_{\perp}}{(x-z)_{\perp}^{2}(z-y)_{\perp}^{2}}\left[\operatorname{Tr}\left\{U_{z} U_{y}^{\dagger} U_{x} \mathcal{X}_{1 x z}^{\dagger}\right\}+\frac{1}{N_{c}} \operatorname{Tr}\left\{U_{y}^{\dagger} U_{x}\right\} \mathcal{H}_{1 z x}^{+}\right]\right. \\
& \left.\quad-\frac{(\vec{x}-\vec{z}) \times(\vec{y}-\vec{z})}{(x-z)_{\perp}^{2}(z-y)_{\perp}^{2}}\left[\operatorname{Tr}\left\{U_{z} U_{y}^{\dagger} U_{x} \mathcal{X}_{5 x z}^{\dagger}\right\}-\frac{1}{N_{c}} \operatorname{Tr}\left\{U_{y}^{\dagger} U_{x}\right\} \mathcal{H}_{5 z x}^{+}\right]\right\} .
\end{aligned}
$$


With $Q_{5 x}$ we have

$$
\begin{aligned}
& \left\langle\operatorname{Tr}\left\{U_{x} U_{y}^{\dagger}\right\} Q_{5 x}\right\rangle_{\text {Figure 13d }} \\
& =-\frac{\alpha_{s}}{4 \pi^{2}} \int_{0}^{+\infty} \frac{d \alpha}{\alpha} \int d^{2} z\left\{\frac{(x-z, z-y)_{\perp}}{(x-z)_{\perp}^{2}(z-y)_{\perp}^{2}}\left[\operatorname{Tr}\left\{U_{z} U_{y}^{\dagger} U_{x} \mathcal{X}_{5 x z}^{\dagger}\right\}-\frac{1}{N_{c}} \operatorname{Tr}\left\{U_{y}^{\dagger} U_{x}\right\} \mathcal{H}_{5 z x}^{+}\right]\right. \\
& \left.\quad+\frac{(\vec{x}-\vec{z}) \times(\vec{y}-\vec{z})}{(x-z)_{\perp}^{2}(z-y)_{\perp}^{2}}\left[\operatorname{Tr}\left\{U_{z} U_{y}^{\dagger} U_{x} \mathcal{X}_{1 x z}^{\dagger}\right\}+\frac{1}{N_{c}} \operatorname{Tr}\left\{U_{y}^{\dagger} U_{x}\right\} \mathcal{H}_{1 z x}^{+}\right]\right\} .
\end{aligned}
$$

Let us sum up diagrams in figure 13. Summing (6.85), (6.89), (6.91), and (6.95) we have

$$
\begin{aligned}
&\left\langle\operatorname{Tr}\left\{U_{x} U_{y}^{\dagger}\right\} Q_{1 x}\right\rangle_{\text {Figure } 13} \\
&=\frac{\alpha_{s}}{4 \pi^{2}} \int_{0}^{+\infty} \frac{d \alpha}{\alpha} \int d^{2} z\left\{\frac { 1 } { ( x - z ) _ { \perp } ^ { 2 } } \left[\operatorname{Tr}\left\{U_{x} U_{y}^{\dagger} U_{z} \mathcal{X}_{1 z x}^{\dagger}\right\}\right.\right. \\
&+\left.\operatorname{Tr}\left\{U_{z} U_{y}^{\dagger} U_{x} \mathcal{X}_{1 x z}^{\dagger}\right\}+\frac{1}{N_{c}} \operatorname{Tr}\left\{U_{x} U_{y}^{\dagger}\right\}\left(\mathcal{H}_{1 x z}^{-}+\mathcal{H}_{1 z x}^{+}\right)\right]+\frac{(x-z, z-y)_{\perp}}{(y-z)_{\perp}^{2}(x-z)_{\perp}^{2}} \\
& \times\left[\operatorname{Tr}\left\{U_{x} U_{y}^{\dagger} U_{z} \mathcal{X}_{1 z x}^{\dagger}\right\}+\operatorname{Tr}\left\{U_{z} U_{y}^{\dagger} U_{x} \mathcal{X}_{1 x z}^{\dagger}\right\}+\frac{1}{N_{c}} \operatorname{Tr}\left\{U_{x} U_{y}^{\dagger}\right\}\left(\mathcal{H}_{1 x z}^{-}+\mathcal{H}_{1 z x}^{+}\right)\right] \\
&+\frac{(\vec{x}-\vec{z}) \times(\vec{y}-\vec{z})}{(y-z)_{\perp}^{2}(x-z)_{\perp}^{2}}\left[\operatorname{Tr}\left\{U_{x} U_{y}^{\dagger} U_{z} \mathcal{X}_{5 z x}^{\dagger}\right\}-\operatorname{Tr}\left\{U_{z} U_{y}^{\dagger} U_{x} \mathcal{X}_{5 x z}^{\dagger}\right\}\right. \\
&\left.\left.-\frac{1}{N_{c}} \operatorname{Tr}\left\{U_{x} U_{y}^{\dagger}\right\}\left(\mathcal{H}_{5 x z}^{-}-\mathcal{H}_{5 z x}^{+}\right)\right]\right\} .
\end{aligned}
$$

Summing, instead, (6.86), (6.90), (6.92), and (6.96) we have

$$
\begin{aligned}
\left\langle\operatorname{Tr}\left\{U_{x} U_{y}^{\dagger}\right\} Q_{5 x}\right\rangle_{\text {Figure } 13} & =\frac{\alpha_{s}}{4 \pi^{2}} \int_{0}^{+\infty} \frac{d \alpha}{\alpha} \int d^{2} z\left\{\frac { 1 } { ( x - z ) _ { \perp } ^ { 2 } } \left[\operatorname{Tr}\left\{U_{x} U_{y}^{\dagger} U_{z} \mathcal{X}_{5 z x}^{\dagger}\right\}\right.\right. \\
& \left.+\operatorname{Tr}\left\{U_{z} U_{y}^{\dagger} U_{x} \mathcal{X}_{5 x z}^{\dagger}\right\}-\frac{1}{N_{c}} \operatorname{Tr}\left\{U_{y}^{\dagger} U_{x}\right\}\left(\mathcal{H}_{5 x z}^{-}+\mathcal{H}_{5 z x}^{+}\right)\right]+\frac{(x-z, z-y)_{\perp}}{(y-z)_{\perp}^{2}(x-z)_{\perp}^{2}} \\
& \times\left[\operatorname{Tr}\left\{U_{x} U_{y}^{\dagger} U_{z} \mathcal{X}_{5 z x}^{\dagger}\right\}+\operatorname{Tr}\left\{U_{z} U_{y}^{\dagger} U_{x} \mathcal{X}_{5 x z}^{\dagger}\right\}-\frac{1}{N_{c}} \operatorname{Tr}\left\{U_{x} U_{y}^{\dagger}\right\}\left(\mathcal{H}_{5 x z}^{-}+\mathcal{H}_{5 z x}^{+}\right)\right] \\
& +\frac{(\vec{x}-\vec{z}) \times(\vec{y}-\vec{z})}{(y-z)_{\perp}^{2}(x-z)_{\perp}^{2}}\left[\operatorname{Tr}\left\{U_{z} U_{y}^{\dagger} U_{x} \mathcal{X}_{1 x z}^{\dagger}\right\}-\operatorname{Tr}\left\{U_{x} U_{y}^{\dagger} U_{z} \mathcal{X}_{1 z x}^{\dagger}\right\}\right. \\
& \left.\left.+\frac{1}{N_{c}} \operatorname{Tr}\left\{U_{x} U_{y}^{\dagger}\right\}\left(\mathcal{H}_{1 z x}^{+}-\mathcal{H}_{1 x z}^{-}\right)\right]\right\} .
\end{aligned}
$$

\section{$7 \quad$ Summary of evolution equations}

In this section we summarize the evolution equations we have calculated. As already discussed in the previous sections, the operators of which we want to find the evolution 
equations are the ones proportional to the impact factors. The operator proportional to the impact factor $\mathcal{I}_{1}^{\mu \nu}$ is

$$
\operatorname{Tr}\left\{\hat{\mathcal{Q}}_{1 x} \hat{U}_{y}^{\dagger}\right\}=\frac{1}{2} \operatorname{Tr}\left\{\hat{U}_{y}^{\dagger} \hat{U}_{x}\right\} \hat{Q}_{1 x}-\frac{1}{2 N_{c}} \operatorname{Tr}\left\{\hat{U}_{y}^{\dagger} \hat{\tilde{Q}}_{1 x}\right\}
$$

The operator proportional to the impact factor $\mathcal{I}_{5}^{\mu \nu}$ is, for flavor singlet,

$$
\begin{aligned}
& \operatorname{Tr}\left\{\left(\hat{\mathcal{Q}}_{5 z_{2}}+\hat{\mathcal{F}}_{z_{2}}\right) \hat{U}_{z_{1}}^{\dagger}\right\}+\operatorname{Tr}\left\{\left(\hat{\mathcal{Q}}_{5 z_{2}}+\hat{\mathcal{F}}_{z_{2}}^{\dagger}\right) \hat{U}_{z_{1}}\right\} \\
& =\operatorname{Tr}\left\{\hat{U}_{z_{1}}^{\dagger} \hat{U}_{z_{2}}\right\} \hat{Q}_{5 z_{2}}+\operatorname{Tr}\left\{\hat{U}_{z_{1}} \hat{U}_{z_{2}}\right\} \hat{Q}_{5 z_{2}}^{\dagger} \\
& \quad-\frac{1}{N_{c}} \operatorname{Tr}\left\{\hat{U}_{z_{1}}^{\dagger}\left(\hat{\tilde{Q}}_{5 z_{2}}-2 N_{c} \hat{\mathcal{F}}_{z_{2}}\right)\right\}-\frac{1}{N_{c}} \operatorname{Tr}\left\{\hat{U}_{z_{1}}\left(\hat{\tilde{Q}}_{5 z_{2}}^{\dagger}-2 N_{c} \hat{\mathcal{F}}_{z_{2}}^{\dagger}\right)\right\}
\end{aligned}
$$

and for flavor non-singlet case

$$
\begin{aligned}
& \operatorname{Tr}\left\{\hat{\mathcal{Q}}_{5 z_{2}} \hat{U}_{z_{1}}^{\dagger}\right\}+\operatorname{Tr}\left\{\hat{\mathcal{Q}}_{5 z_{2}} \hat{U}_{z_{1}}\right\} \\
& =\operatorname{Tr}\left\{\hat{U}_{z_{1}}^{\dagger} \hat{U}_{z_{2}}\right\} \hat{Q}_{5 z_{2}}+\operatorname{Tr}\left\{\hat{U}_{z_{1}} \hat{U}_{z_{2}}\right\} \hat{Q}_{5 z_{2}}^{\dagger}-\frac{1}{N_{c}} \operatorname{Tr}\left\{\hat{U}_{z_{1}}^{\dagger} \hat{\tilde{Q}}_{5 z_{2}}\right\}-\frac{1}{N_{c}} \operatorname{Tr}\left\{\hat{U}_{z_{1}} \hat{\tilde{Q}}_{5 z_{2}}^{\dagger}\right\}
\end{aligned}
$$

The evolution equations that we are going to collect in this sections are not strictly speaking evolution equations of the type $\frac{d}{d \eta} \mathcal{O}=K \otimes \mathcal{O}$ like the BK equation (2.20). The reason lies in the fact that there are divergences of the type $\frac{d^{2} z}{(x-z)_{\perp}^{2}}$ which are responsible for the double log contributions peculiar of the polarized structure functions at small- $x_{B}$ and of the unpolarized quark structure functions as well. In this work we limit ourself to calculate all the diagrams at one loop level, and properly analyze the double log of energy contributions in a separate publication. Actually, in appendix F, eq. (F.13), we obtain the double log of energy evolution equation which agree with the one calculated in refs. $[5,7,8]$, but as we will argue in the conclusions, the evolution equations will differ when written in terms of the operators $Q_{5 x}$ and $\tilde{Q}_{5 x}$.

\section{$7.1 Q_{1 x}$ and $\tilde{Q}_{1 x}$ operators}

Here we collect the evolution equations for $Q_{1 x}$ and $\tilde{Q}_{1 x}$.

Summing (6.13), (6.22), and (6.97) we have

$$
\begin{aligned}
\langle & \left.\operatorname{Tr}\left\{U_{y}^{\dagger} U_{x}\right\} Q_{1 x}\right\rangle \\
= & \frac{\alpha_{s}}{4 \pi^{2}} \int_{0}^{+\infty} \frac{d \alpha}{\alpha} \int d^{2} z\left\{\frac{\operatorname{Tr}\left\{U_{y}^{\dagger} U_{x}\right\}}{(x-z)_{\perp}^{2}}\left[\operatorname{Tr}\left\{U_{x}^{\dagger} U_{z}\right\} Q_{1 z}-\frac{1}{N_{c}} \operatorname{Tr}\left\{U_{x}^{\dagger} \tilde{Q}_{1 z}\right\}\right]\right. \\
& +\frac{2 Q_{1 x}(x-y)_{\perp}^{2}}{(x-z)_{\perp}^{2}(y-z)_{\perp}^{2}}\left[\operatorname{Tr}\left\{U_{x} U_{z}^{\dagger}\right\} \operatorname{Tr}\left\{U_{z} U_{y}^{\dagger}\right\}-N_{c} \operatorname{Tr}\left\{U_{x} U_{y}^{\dagger}\right\}\right] \\
& +\frac{1}{(x-z)_{\perp}^{2}}\left[\operatorname{Tr}\left\{U_{x} U_{y}^{\dagger} U_{z} \mathcal{X}_{1 z x}^{\dagger}\right\}+\operatorname{Tr}\left\{U_{z} U_{y}^{\dagger} U_{x} \mathcal{X}_{1 x z}^{\dagger}\right\}+\frac{1}{N_{c}} \operatorname{Tr}\left\{U_{x} U_{y}^{\dagger}\right\}\left(\mathcal{H}_{1 x z}^{-}+\mathcal{H}_{1 z x}^{+}\right)\right] \\
& +\frac{(x-z, z-y)_{\perp}}{(y-z)_{\perp}^{2}(x-z)_{\perp}^{2}}\left[\operatorname{Tr}\left\{U_{x} U_{y}^{\dagger} U_{z} \mathcal{X}_{1 z x}^{\dagger}\right\}+\operatorname{Tr}\left\{U_{z} U_{y}^{\dagger} U_{x} \mathcal{X}_{1 x z}^{\dagger}\right\}\right. \\
& \left.+\frac{1}{N_{c}} \operatorname{Tr}\left\{U_{x} U_{y}^{\dagger}\right\}\left(\mathcal{H}_{1 x z}^{-}+\mathcal{H}_{1 z x}^{+}\right)\right]+\frac{(\vec{x}-\vec{z}) \times(\vec{y}-\vec{z})}{(y-z)_{\perp}^{2}(x-z)_{\perp}^{2}}\left[\operatorname{Tr}\left\{U_{x} U_{y}^{\dagger} U_{z} \mathcal{X}_{5 z x}^{\dagger}\right\}\right. \\
& \left.\left.-\operatorname{Tr}\left\{U_{z} U_{y}^{\dagger} U_{x} \mathcal{X}_{5 x z}^{\dagger}\right\}-\frac{1}{N_{c}} \operatorname{Tr}\left\{U_{x} U_{y}^{\dagger}\right\}\left(\mathcal{H}_{5 x z}^{-}-\mathcal{H}_{5 z x}^{+}\right)\right]\right\} .
\end{aligned}
$$


Summing eqs. (6.20), (6.28), (6.80)

$$
\begin{aligned}
& \left\langle\operatorname{Tr}\left\{U_{y}^{\dagger} \tilde{Q}_{1 x}\right\}\right\rangle \\
& =\frac{\alpha_{s}}{4 \pi^{2}} \int_{0}^{+\infty} \frac{d \alpha}{\alpha} \int d^{2} z\left\{\frac { 1 } { ( x - z ) _ { \perp } ^ { 2 } } \left[\operatorname{Tr}\left\{U_{z} U_{y}^{\dagger}\right\}\left(Q_{1 z}-\mathcal{H}_{1 x z}^{+}-\mathcal{H}_{1 z x}^{-}\right)\right.\right. \\
& \left.+\frac{1}{N_{c}} \operatorname{Tr}\left\{U_{y}^{\dagger}\left(\mathcal{X}_{1 x z}+\mathcal{X}_{1 z x}-\tilde{Q}_{1 z}\right)\right\}\right] \\
& -\frac{(x-z, z-y)}{(y-z)_{\perp}^{2}(z-x)_{\perp}^{2}}\left[\operatorname{Tr}\left\{U_{z} U_{y}^{\dagger}\right\}\left(\mathcal{H}_{1 x z}^{+}+\mathcal{H}_{1 z x}^{-}\right)-\frac{1}{N_{c}} \operatorname{Tr}\left\{U_{y}^{\dagger}\left(\mathcal{X}_{1 x z}+\mathcal{X}_{1 z x}\right)\right\}\right] \\
& -\frac{(\vec{x}-\vec{z}) \times(\vec{y}-\vec{z})}{(y-z)_{\perp}^{2}(z-x)_{\perp}^{2}}\left[\operatorname{Tr}\left\{U_{z} U_{y}^{\dagger}\right\}\left(\mathcal{H}_{5 z x}^{-}-\mathcal{H}_{5 x z}^{+}\right)+\frac{1}{N_{c}} \operatorname{Tr}\left\{U_{y}^{\dagger}\left(\mathcal{X}_{5 x z}-\mathcal{X}_{5 z x}\right)\right\}\right] \\
& \left.+\frac{2(x-y)_{\perp}^{2}}{(x-z)_{\perp}^{2}(z-y)_{\perp}^{2}}\left(\operatorname{Tr}\left\{U_{z} U_{y}^{\dagger}\right\} \operatorname{Tr}\left\{U_{z}^{\dagger} \tilde{Q}_{1 x}\right\}-N_{c} \operatorname{Tr}\left\{U_{y}^{\dagger} \tilde{Q}_{1 x}\right\}\right)\right\} .
\end{aligned}
$$

For operator $\hat{\mathcal{Q}}_{1}\left(x_{\perp}\right)$, instead, we have to sum (6.28) (with $\tilde{Q}_{1}\left(x_{\perp}\right)$ replaced by $\left.\mathcal{Q}_{1}\left(x_{\perp}\right)\right),(\mathrm{F} .8)$, and (F.22) and get

$$
\begin{aligned}
\langle & \left.\operatorname{Tr}\left\{\mathcal{Q}_{1 x} U_{y}^{\dagger}\right\}\right\rangle \\
= & \frac{\alpha_{s}}{2 \pi^{2}} \int_{0}^{+\infty} \frac{d \alpha}{\alpha} \int d^{2} z\left\{\frac{1}{2} \frac{1}{(x-z)_{\perp}^{2}}\left(\operatorname{Tr}\left\{U_{x}^{\dagger} \mathcal{Q}_{1 x}\right\} \operatorname{Tr}\left\{U_{y}^{\dagger} U_{x}\right\}-\frac{1}{N_{c}} \operatorname{Tr}\left\{\mathcal{Q}_{1 z} U_{y}^{\dagger}\right\}\right)\right. \\
& +\frac{(x-y)_{\perp}^{2}}{(x-z)_{\perp}^{2}(y-z)_{\perp}^{2}}\left(\operatorname{Tr}\left\{U_{z}^{\dagger} \mathcal{Q}_{1 x}\right\} \operatorname{Tr}\left\{U_{y}^{\dagger} U_{z}\right\}-N_{c} \operatorname{Tr}\left\{U_{y}^{\dagger} \mathcal{Q}_{1 x}\right\}\right) \\
& -\frac{1}{4}\left[\frac{(x-z, z-y)}{(x-z)_{\perp}^{2}(y-z)_{\perp}^{2}}+\frac{1}{(x-z)_{\perp}^{2}}\right]\left[\operatorname{Tr}\left\{U_{x} U_{y}^{\dagger} U_{z} \mathcal{X}_{1 z x}^{\dagger}\right\}+\operatorname{Tr}\left\{U_{z} U_{y}^{\dagger} U_{x} \mathcal{X}_{1 z x}^{\dagger}\right\}\right. \\
& +\frac{1}{N_{c}}\left(\operatorname{Tr}\left\{U_{y}^{\dagger} U_{z}\right\}\left(\mathcal{H}_{1 x z}^{+}+\mathcal{H}_{1 z x}^{-}\right)+\operatorname{Tr}\left\{U_{y}^{\dagger} U_{x}\right\}\left(\mathcal{H}_{1 z x}^{+}+\mathcal{H}_{1 x z}^{-}\right)\right) \\
& \left.-\frac{1}{N_{c}^{2}}\left(\operatorname{Tr}\left\{U_{z} U_{y}^{\dagger} U_{x} \mathcal{X}_{1 z x}^{\dagger}\right\}+\operatorname{Tr}\left\{U_{x} U_{y}^{\dagger} U_{z} \mathcal{X}_{1 x z}^{\dagger}\right\}\right)\right] \\
& +\frac{1}{4} \frac{(\vec{x}-\vec{z}) \times(\vec{y}-\vec{z})}{(x-z)_{\perp}^{2}(y-z)_{\perp}^{2}}\left[\operatorname{Tr}\left\{U_{z} U_{y}^{\dagger} U_{x} \mathcal{X}_{5 x z}^{\dagger}\right\}-\operatorname{Tr}\left\{U_{x} U_{y}^{\dagger} U_{z} \mathcal{X}_{5 z x}^{\dagger}\right\}\right. \\
& +\frac{1}{N_{c}}\left(\operatorname{Tr}\left\{U_{y}^{\dagger} U_{z}\right\}\left(\mathcal{H}_{5 x z}^{+}-\mathcal{H}_{5 z x}^{-}\right)+\operatorname{Tr}\left\{U_{y}^{\dagger} U_{x}\right\}\left(\mathcal{H}_{5 x z}^{-}-\mathcal{H}_{5 z x}^{+}\right)\right) \\
& \left.\left.+\frac{1}{N_{c}^{2}}\left(\operatorname{Tr}\left\{U_{z} U_{y}^{\dagger} U_{x} \mathcal{X}_{5 z x}^{\dagger}\right\}-\operatorname{Tr}\left\{U_{x} U_{y}^{\dagger} U_{z} \mathcal{X}_{5 x z}^{\dagger}\right\}\right)\right]\right\} .
\end{aligned}
$$

\section{2 $Q_{5 x}, \tilde{Q}_{5 x}$, and $\mathcal{F}_{x}$ operators: flavor singlet}

Here we collect the evolution equations for operators $Q_{5 x}, \tilde{Q}_{5 x}$, and $\mathcal{F}_{x}$ in the flavor singlet case. 
Summing (6.14), (6.22) (with $Q_{1}$ replaced by $Q_{5}$ ), and (6.98) we obtain

$$
\begin{aligned}
&\left\langle\operatorname{Tr}\left\{U_{y}^{\dagger} U_{x}\right\} Q_{5 x}\right\rangle \\
&=\frac{\alpha_{s}}{4 \pi^{2}} \int_{0}^{+\infty} \frac{d \alpha}{\alpha} \int d^{2} z\left\{\frac{\operatorname{Tr}\left\{U_{y}^{\dagger} U_{x}\right\}}{(x-z)_{\perp}^{2}}\left[\operatorname{Tr}\left\{U_{x}^{\dagger} U_{z}\right\} Q_{5 z}-\frac{1}{N_{c}} \operatorname{Tr}\left\{U_{x}^{\dagger}\left(\tilde{Q}_{5 z}-2 N_{c} \mathcal{F}_{z}\right)\right\}\right]\right. \\
&+\frac{2 Q_{5 x}(x-y)_{\perp}^{2}}{(x-z)_{\perp}^{2}(y-z)_{\perp}^{2}}\left[\operatorname{Tr}\left\{U_{x} U_{z}^{\dagger}\right\} \operatorname{Tr}\left\{U_{z} U_{y}^{\dagger}\right\}-N_{c} \operatorname{Tr}\left\{U_{x} U_{y}^{\dagger}\right\}\right] \\
&+\frac{1}{(x-z)_{\perp}^{2}}\left[\operatorname{Tr}\left\{U_{x} U_{y}^{\dagger} U_{z} \mathcal{X}_{5 z x}^{\dagger}\right\}+\operatorname{Tr}\left\{U_{z} U_{y}^{\dagger} U_{x} \mathcal{X}_{5 x z}^{\dagger}\right\}-\frac{1}{N_{c}} \operatorname{Tr}\left\{U_{y}^{\dagger} U_{x}\right\}\left(\mathcal{H}_{5 x z}^{-}+\mathcal{H}_{5 z x}^{+}\right)\right] \\
&+\frac{(x-z, z-y)_{\perp}}{(y-z)_{\perp}^{2}(x-z)_{\perp}^{2}}\left[\operatorname{Tr}\left\{U_{x} U_{y}^{\dagger} U_{z} \mathcal{X}_{5 z x}^{\dagger}\right\}+\operatorname{Tr}\left\{U_{z} U_{y}^{\dagger} U_{x} \mathcal{X}_{5 x z}^{\dagger}\right\}\right. \\
&\left.-\frac{1}{N_{c}} \operatorname{Tr}\left\{U_{x} U_{y}^{\dagger}\right\}\left(\mathcal{H}_{5 x z}^{-}+\mathcal{H}_{5 z x}^{+}\right)\right]+\frac{(\vec{x}-\vec{z}) \times(\vec{y}-\vec{z})}{(y-z)_{\perp}^{2}(x-z)_{\perp}^{2}}\left[\operatorname{Tr}\left\{U_{z} U_{y}^{\dagger} U_{x} \mathcal{X}_{1 x z}^{\dagger}\right\}\right. \\
&\left.-\operatorname{Tr}\left\{\operatorname{Tr}\left\{U_{x} U_{y}^{\dagger} U_{z} \mathcal{X}_{1 z x}^{\dagger}\right\}+\frac{1}{N_{c}} \operatorname{Tr}\left\{U_{x} U_{y}^{\dagger}\right\}\left(\mathcal{H}_{1 z x}^{+}-\mathcal{H}_{1 x z}^{-}\right)\right]\right\}
\end{aligned}
$$

The evolution equation for operator $\operatorname{Tr}\left\{\hat{U}_{y}^{\dagger} \hat{\tilde{Q}}_{5 x}\right\}$ is obtained summing eqs. (6.28) (with $\tilde{Q}_{1 x}$ replaced by $\left.\tilde{Q}_{5 x}\right),(6.21)$, and (6.81). Thus, we have

$$
\begin{aligned}
&\left\langle\operatorname{Tr}\left\{U_{y}^{\dagger} \tilde{Q}_{5 x}\right\}\right\rangle \\
&=\frac{\alpha_{s}}{4 \pi^{2}} \int_{0}^{+\infty} \frac{d \alpha}{\alpha} \int d^{2} z\left\{\frac { 1 } { ( x - z ) _ { \perp } ^ { 2 } } \left[\operatorname{Tr}\left\{U_{y}^{\dagger} U_{z}\right\}\left(Q_{5 z}-\mathcal{H}_{5 x z}^{+}-\mathcal{H}_{5 z x}^{-}\right)\right.\right. \\
&\left.+\frac{1}{N_{c}} \operatorname{Tr}\left\{U_{y}^{\dagger}\left(\mathcal{X}_{5 x z}+\mathcal{X}_{5 z x}-\tilde{Q}_{5 z}+N_{c} \mathcal{F}_{z}\right)\right\}\right] \\
&\left.-\frac{(x-z, z-y)}{(y-z)_{\perp}^{2}(z-x)_{\perp}^{2}}\left[\operatorname{Tr}\left\{U_{z} U_{y}^{\dagger}\right\}\left(\mathcal{H}_{5 x z}^{+}+\mathcal{H}_{5 z x}^{-}\right)-\frac{1}{N_{c}} \operatorname{Tr}\left\{U_{y}^{\dagger}\left(\mathcal{X}_{5 x z}+\mathcal{X}_{5 z x}\right)\right\}\right)\right] \\
&-\frac{(\vec{x}-\vec{z}) \times(\vec{y}-\vec{z})}{(y-z)_{\perp}^{2}(z-x)_{\perp}^{2}}\left[\operatorname{Tr}\left\{U_{z} U_{y}^{\dagger}\right\}\left(\mathcal{H}_{1 x z}^{+}-\mathcal{H}_{1 z x}^{-}\right)+\frac{1}{N_{c}} \operatorname{Tr}\left\{U_{y}^{\dagger}\left(\mathcal{X}_{1 z x}-\mathcal{X}_{1 x z}\right)\right\}\right] \\
&\left.+\frac{2(x-y)_{\perp}^{2}}{(x-z)_{\perp}^{2}(z-y)_{\perp}^{2}}\left(\operatorname{Tr}\left\{U_{z} U_{y}^{\dagger}\right\} \operatorname{Tr}\left\{U_{z}^{\dagger} \tilde{Q}_{5 x}\right\}-N_{c} \operatorname{Tr}\left\{U_{y}^{\dagger} \tilde{Q}_{5 x}\right\}\right)\right\}
\end{aligned}
$$


Evolution equation for operator $\hat{\mathcal{F}}\left(z_{\perp}\right)$ is the sum of eqs. (6.28) (with $\hat{\widetilde{Q}}_{1 x}$ replaced by $\left.\hat{\mathcal{F}}_{x}\right)$, and $(6.50)$

$$
\begin{aligned}
\langle & \left.\operatorname{Tr}\left\{\mathcal{F}_{x} U_{y}^{\dagger}\right\}\right\rangle \\
= & \frac{\alpha_{s}}{4 \pi^{2}} \int_{0}^{+\infty} \frac{d \alpha}{\alpha} \int d^{2} z\left\{\frac { ( \vec { x } - \vec { z } ) \times ( \vec { z } - \vec { y } ) } { ( x - z ) _ { \perp } ^ { 2 } ( y - z ) _ { \perp } ^ { 2 } } \left[\operatorname{Tr}\left\{U_{y}^{\dagger} \tilde{Q}_{1 z}\right\} \operatorname{Tr}\left\{U_{z}^{\dagger} U_{x}\right\}-\operatorname{Tr}\left\{U_{x} \tilde{Q}_{1 z}^{\dagger}\right\} \operatorname{Tr}\left\{U_{y}^{\dagger} U_{z}\right\}\right.\right. \\
& +\frac{1}{N_{c}}\left(\operatorname{Tr}\left\{U_{x} U_{y}^{\dagger} \tilde{Q}_{1 z} U_{z}^{\dagger}\right\}+\operatorname{Tr}\left\{U_{y}^{\dagger} U_{x} U_{z}^{\dagger} \tilde{Q}_{1 z}\right\}-\operatorname{Tr}\left\{U_{x} U_{y}^{\dagger} U_{z} \tilde{Q}_{1 z}^{\dagger}\right\}-\operatorname{Tr}\left\{U_{y}^{\dagger} U_{x} \tilde{Q}_{1 z}^{\dagger} U_{z}\right\}\right) \\
& \left.+\frac{1}{N_{c}^{2}} \operatorname{Tr}\left\{U_{y}^{\dagger} U_{x}\right\}\left(Q_{1 z}^{\dagger}-Q_{1 z}\right)\right]+\left(\frac{(x-z, z-y)}{(x-z)_{\perp}^{2}(y-z)_{\perp}^{2}}+\frac{1}{(x-z)_{\perp}^{2}}\right) \\
& \times\left[\operatorname{Tr}\left\{U_{y}^{\dagger}\left(2 \mathcal{F}_{z}-\tilde{Q}_{5 z}\right)\right\} \operatorname{Tr}\left\{U_{z}^{\dagger} U_{x}\right\}+\operatorname{Tr}\left\{U_{x}\left(2 \mathcal{F}_{z}^{\dagger}-\tilde{Q}_{5 z}^{\dagger}\right)\right\} \operatorname{Tr}\left\{U_{y}^{\dagger} U_{z}\right\}\right. \\
& +\frac{1}{N_{c}}\left(\operatorname{Tr}\left\{U_{x} U_{y}^{\dagger} U_{z} \tilde{Q}_{5 z}^{\dagger}\right\}+\operatorname{Tr}\left\{U_{y}^{\dagger} U_{x} \tilde{Q}_{5 z}^{\dagger} U_{z}\right\}+\operatorname{Tr}\left\{U_{x} U_{y}^{\dagger} \tilde{Q}_{5 z} U_{z}^{\dagger}\right\}+\operatorname{Tr}\left\{U_{y}^{\dagger} U_{x} U_{z}^{\dagger} \tilde{Q}_{5 z}\right\}\right) \\
& \left.-\frac{1}{N_{c}^{2}} \operatorname{Tr}\left\{U_{y}^{\dagger} U_{x}\right\}\left(Q_{5 z}+Q_{5 z}^{\dagger}\right)\right]+8 \pi^{2} \int d^{2} q_{1} \frac{e^{i\left(q_{1}, y-z\right)}-e^{i\left(q_{1}, x-z\right)}}{q_{1 \perp}^{2}} \delta^{(2)}(z-x) \\
& \times\left[\operatorname{Tr}\left\{U_{x} U_{z}^{\dagger}\right\} \operatorname{Tr}\left\{U_{y}^{\dagger} \mathcal{F}_{z}\right\}+\operatorname{Tr}\left\{U_{u}^{\dagger} U_{z}\right\} \operatorname{Tr}\left\{U_{x} \mathcal{F}_{z}^{\dagger}\right\}\right] \\
& \left.+\frac{2(x-y)_{\perp}^{2}}{(x-z)_{\perp}^{2}(z-y)_{\perp}^{2}}\left(\operatorname{Tr}\left\{U_{z} U_{y}^{\dagger}\right\} \operatorname{Tr}\left\{U_{z}^{\dagger} \mathcal{F}_{x}\right\}-N_{c} \operatorname{Tr}\left\{U_{y}^{\dagger} \mathcal{F}_{x}\right\}\right)\right\}
\end{aligned}
$$

Evolution equation (7.9) should clarify why we needed evolution equations (7.4), (7.5), (7.7), and (7.8).

We can also write done the evolution equation for operator $\operatorname{Tr}\left\{\hat{\mathcal{Q}}_{5 x} \hat{U}_{y}^{\dagger}\right\}$ summing eqs. (6.28) (with $\hat{\tilde{Q}}_{1 x}$ replaced by $\hat{\mathcal{Q}}_{5 x}$ ), (F.6), and (F.30). We have

$$
\begin{aligned}
\langle & \left.\operatorname{Tr}\left\{\mathcal{Q}_{5 x} U_{y}^{\dagger}\right\}\right\rangle \\
= & \frac{\alpha_{s}}{2 \pi^{2}} \int_{0}^{+\infty} \frac{d \alpha}{\alpha} \int d^{2} z\left\{\frac{1}{2} \frac{1}{(x-z)_{\perp}^{2}}\left[\operatorname{Tr}\left\{U_{x}^{\dagger}\left(\mathcal{Q}_{5 z}+\mathcal{F}_{z}\right)\right\} \operatorname{Tr}\left\{U_{y}^{\dagger} U_{x}\right\}-\frac{1}{N_{c}} \operatorname{Tr}\left\{\left(\mathcal{Q}_{5 z}+\mathcal{F}_{z}\right) U_{y}^{\dagger}\right\}\right]\right. \\
& +\frac{(x-y)_{\perp}^{2}}{(x-z)_{\perp}^{2}(y-z)_{\perp}^{2}}\left[\operatorname{Tr}\left\{\mathcal{Q}_{5 x} U_{z}^{\dagger}\right\} \operatorname{Tr}\left\{U_{z} U_{y}^{\dagger}\right\}-N_{c} \operatorname{Tr}\left\{U_{y}^{\dagger} \mathcal{Q}_{5 x}\right\}\right] \\
& +\frac{1}{4}\left[\frac{(x-z, z-y)}{(x-z)_{\perp}^{2}(y-z)_{\perp}^{2}}+\frac{1}{(x-z)_{\perp}^{2}}\right]\left[\operatorname{Tr}\left\{U_{x} U_{y}^{\dagger} U_{z} \mathcal{X}_{5 z x}^{\dagger}\right\}+\operatorname{Tr}\left\{U_{z} U_{y}^{\dagger} U_{x} \mathcal{X}_{5 x z}^{\dagger}\right\}\right. \\
& -\frac{1}{N_{c}}\left(\operatorname{Tr}\left\{U_{y}^{\dagger} U_{z}\right\}\left(\mathcal{H}_{5 x z}^{+}+\mathcal{H}_{5 z x}^{-}\right)+\operatorname{Tr}\left\{U_{y}^{\dagger} U_{x}\right\}\left(\mathcal{H}_{5 x z}^{-}+\mathcal{H}_{5 z x}^{+}\right)\right) \\
& -\frac{1}{N_{c}^{2}}\left(\operatorname{Tr}\left\{U_{z} U_{y}^{\dagger} U_{x} \mathcal{X}_{5 z x}^{\dagger}\right\}+\operatorname{Tr}\left\{U_{x} U_{y}^{\dagger} U_{z} \mathcal{X}_{5 x z}^{\dagger}\right\}\right] \\
& +\frac{1}{4} \frac{(\vec{x}-\vec{z}) \times(\vec{y}-\vec{z})}{(x-z)_{\perp}^{2}(y-z)_{\perp}^{2}}\left[\operatorname{Tr}\left\{U_{z} U_{y}^{\dagger} U_{x} \mathcal{X}_{1 x z}^{\dagger}\right\}-\operatorname{Tr}\left\{U_{x} U_{y}^{\dagger} U_{z} \mathcal{X}_{1 z x}^{\dagger}\right\}\right. \\
& -\frac{1}{N_{c}}\left(\operatorname{Tr}\left\{U_{y}^{\dagger} U_{z}\right\}\left(\mathcal{H}_{1 x z}^{+}-\mathcal{H}_{1 z x}^{-}\right)+\operatorname{Tr}\left\{U_{y}^{\dagger} U_{x}\right\}\left(\mathcal{H}_{1 x z}^{-}-\mathcal{H}_{1 z x}^{+}\right)\right) \\
& \left.\left.+\frac{1}{N_{c}^{2}}\left(\operatorname{Tr}\left\{U_{z} U_{y}^{\dagger} U_{x} \mathcal{X}_{1 z x}^{\dagger}\right\}-\operatorname{Tr}\left\{U_{x} U_{y}^{\dagger} U_{z} \mathcal{X}_{1 x z}^{\dagger}\right\}\right)\right]\right\}
\end{aligned}
$$


In eqs. (7.7), (7.8), (7.9), and (7.10) we have again mixing between odd operators which carry the subscript 5 , and even operators with subscript 1 .

The evolution equation of operators in the adjoint representation $\hat{\mathcal{Q}}_{1}\left(x_{\perp}\right)^{a b}, \hat{\mathcal{Q}}_{5}\left(x_{\perp}\right)^{a b}$, and $\hat{\mathcal{F}}^{b a}\left(x_{\perp}\right)$ can be found in appendix F.3.

\section{3 $Q_{5 x}, \tilde{Q}_{5 x}$, and $\mathcal{F}_{x}$ operators: flavor non-singlet}

For flavor non-singlet case we do not have the operator $\hat{\mathcal{F}}\left(x_{\perp}\right)$ because it does not allow flavor exchange. In this case the evolution equations, using results for the singlet case (neglecting operator $\mathcal{F}\left(x_{\perp}\right)$ ), are

$$
\begin{aligned}
& \left\langle\operatorname{Tr}\left\{U_{y}^{\dagger} U_{x}\right\} Q_{5 x}\right\rangle \\
& =\frac{\alpha_{s}}{4 \pi^{2}} \int_{0}^{+\infty} \frac{d \alpha}{\alpha} \int d^{2} z\left\{\frac{\operatorname{Tr}\left\{U_{y}^{\dagger} U_{x}\right\}}{(x-z)_{\perp}^{2}}\left[\operatorname{Tr}\left\{U_{x}^{\dagger} U_{z}\right\} Q_{5 z}-\frac{1}{N_{c}} \operatorname{Tr}\left\{U_{x}^{\dagger} \tilde{Q}_{5 z}\right\}\right]\right. \\
& +\frac{2 Q_{5 x}(x-y)_{\perp}^{2}}{(x-z)_{\perp}^{2}(y-z)_{\perp}^{2}}\left[\operatorname{Tr}\left\{U_{x} U_{z}^{\dagger}\right\} \operatorname{Tr}\left\{U_{z} U_{y}^{\dagger}\right\}-N_{c} \operatorname{Tr}\left\{U_{x} U_{y}^{\dagger}\right\}\right] \\
& +\frac{1}{(x-z)_{\perp}^{2}}\left[\operatorname{Tr}\left\{U_{x} U_{y}^{\dagger} U_{z} \mathcal{X}_{5 z x}^{\dagger}\right\}+\operatorname{Tr}\left\{U_{z} U_{y}^{\dagger} U_{x} \mathcal{X}_{5 x z}^{\dagger}\right\}-\frac{1}{N_{c}} \operatorname{Tr}\left\{U_{y}^{\dagger} U_{x}\right\}\left(\mathcal{H}_{5 x z}^{-}+\mathcal{H}_{5 z x}^{+}\right)\right] \\
& +\frac{(x-z, z-y)_{\perp}}{(y-z)_{\perp}^{2}(x-z)_{\perp}^{2}}\left[\operatorname{Tr}\left\{U_{x} U_{y}^{\dagger} U_{z} \mathcal{X}_{5 z x}^{\dagger}\right\}+\operatorname{Tr}\left\{U_{z} U_{y}^{\dagger} U_{x} \mathcal{X}_{5 x z}^{\dagger}\right\}\right. \\
& \left.-\frac{1}{N_{c}} \operatorname{Tr}\left\{U_{x} U_{y}^{\dagger}\right\}\left(\mathcal{H}_{5 x z}^{-}+\mathcal{H}_{5 z x}^{+}\right)\right]+\frac{(\vec{x}-\vec{z}) \times(\vec{y}-\vec{z})}{(y-z)_{\perp}^{2}(x-z)_{\perp}^{2}}\left[\operatorname{Tr}\left\{U_{z} U_{y}^{\dagger} U_{x} \mathcal{X}_{1 x z}^{\dagger}\right\}\right. \\
& \left.-\operatorname{Tr}\left\{\operatorname{Tr}\left\{U_{x} U_{y}^{\dagger} U_{z} \mathcal{X}_{1 z x}^{\dagger}\right\} \tilde{+} \frac{1}{N_{c}} \operatorname{Tr}\left\{U_{x} U_{y}^{\dagger}\right\}\left(\mathcal{H}_{1 z x}^{+}-\mathcal{H}_{1 x z}^{-}\right)\right]\right\} . \\
& \left\langle\operatorname{Tr}\left\{U_{y}^{\dagger} \tilde{Q}_{5 x}\right\}\right\rangle \\
& =\frac{\alpha_{s}}{4 \pi^{2}} \int_{0}^{+\infty} \frac{d \alpha}{\alpha} \int d^{2} z\left\{\frac { 1 } { ( x - z ) _ { \perp } ^ { 2 } } \left[\operatorname{Tr}\left\{U_{y}^{\dagger} U_{z}\right\}\left(Q_{5 z}-\mathcal{H}_{5 x z}^{+}-\mathcal{H}_{5 z x}^{-}\right)\right.\right. \\
& \left.+\frac{1}{N_{c}} \operatorname{Tr}\left\{U_{y}^{\dagger}\left(\mathcal{X}_{5 x z}+\mathcal{X}_{5 z x}-\tilde{Q}_{5 z}\right)\right\}\right] \\
& \left.-\frac{(x-z, z-y)}{(y-z)_{\perp}^{2}(z-x)_{\perp}^{2}}\left[\operatorname{Tr}\left\{U_{z} U_{y}^{\dagger}\right\}\left(\mathcal{H}_{5 x z}^{+}+\mathcal{H}_{5 z x}^{-}\right)-\frac{1}{N_{c}} \operatorname{Tr}\left\{U_{y}^{\dagger}\left(\mathcal{X}_{5 x z}+\mathcal{X}_{5 z x}\right)\right\}\right)\right] \\
& -\frac{(\vec{x}-\vec{z}) \times(\vec{y}-\vec{z})}{(y-z)_{\perp}^{2}(z-x)_{\perp}^{2}}\left[\operatorname{Tr}\left\{U_{z} U_{y}^{\dagger}\right\}\left(\mathcal{H}_{1 x z}^{+}-\mathcal{H}_{1 z x}^{-}\right)+\frac{1}{N_{c}} \operatorname{Tr}\left\{U_{y}^{\dagger}\left(\mathcal{X}_{1 z x}-\mathcal{X}_{1 x z}\right)\right\}\right] \\
& \left.+\frac{2(x-y)_{\perp}^{2}}{(x-z)_{\perp}^{2}(z-y)_{\perp}^{2}}\left(\operatorname{Tr}\left\{U_{z} U_{y}^{\dagger}\right\} \operatorname{Tr}\left\{U_{z}^{\dagger} \tilde{Q}_{5 x}\right\}-N_{c} \operatorname{Tr}\left\{U_{y}^{\dagger} \tilde{Q}_{5 x}\right\}\right)\right\} \\
& \left\langle\operatorname{Tr}\left\{\mathcal{Q}_{5 x} U_{y}^{\dagger}\right\}\right\rangle \\
& =\frac{\alpha_{s}}{2 \pi^{2}} \int_{0}^{+\infty} \frac{d \alpha}{\alpha} \int d^{2} z\left\{\frac{1}{2} \frac{1}{(x-z)_{\perp}^{2}}\left[\operatorname{Tr}\left\{U_{x}^{\dagger} \mathcal{Q}_{5 z}\right\} \operatorname{Tr}\left\{U_{y}^{\dagger} U_{x}\right\}-\frac{1}{N_{c}} \operatorname{Tr}\left\{\mathcal{Q}_{5 z} U_{y}^{\dagger}\right\}\right]\right. \\
& +\frac{(x-y)_{\perp}^{2}}{(x-z)_{\perp}^{2}(y-z)_{\perp}^{2}}\left[\operatorname{Tr}\left\{U_{z}^{\dagger} \mathcal{Q}_{5 z}\right\} \operatorname{Tr}\left\{U_{y}^{\dagger} U_{z}\right\}-N_{c} \operatorname{Tr}\left\{U_{y}^{\dagger} \mathcal{Q}_{5 z}\right\}\right]
\end{aligned}
$$




$$
\begin{aligned}
& +\frac{1}{4}\left[\frac{(x-z, z-y)}{(x-z)_{\perp}^{2}(y-z)_{\perp}^{2}}+\frac{1}{(x-z)_{\perp}^{2}}\right]\left[\operatorname{Tr}\left\{U_{x} U_{y}^{\dagger} U_{z} \mathcal{X}_{5 z x}^{\dagger}\right\}+\operatorname{Tr}\left\{U_{z} U_{y}^{\dagger} U_{x} \mathcal{X}_{5 x z}^{\dagger}\right\}\right. \\
& -\frac{1}{N_{c}}\left(\operatorname{Tr}\left\{U_{y}^{\dagger} U_{z}\right\}\left(\mathcal{H}_{5 x z}^{+}+\mathcal{H}_{5 z x}^{-}\right)+\operatorname{Tr}\left\{U_{y}^{\dagger} U_{x}\right\}\left(\mathcal{H}_{5 x z}^{-}+\mathcal{H}_{5 z x}^{+}\right)\right) \\
& -\frac{1}{N_{c}^{2}}\left(\operatorname{Tr}\left\{U_{z} U_{y}^{\dagger} U_{x} \mathcal{X}_{5 z x}^{\dagger}\right\}+\operatorname{Tr}\left\{U_{x} U_{y}^{\dagger} U_{z} \mathcal{X}_{5 x z}^{\dagger}\right\}\right]+\frac{1}{4} \frac{(\vec{x}-\vec{z}) \times(\vec{y}-\vec{z})}{(x-z)_{\perp}^{2}(y-z)_{\perp}^{2}}\left[\operatorname{Tr}\left\{U_{z} U_{y}^{\dagger} U_{x} \mathcal{X}_{1 x z}^{\dagger}\right\}\right. \\
& -\operatorname{Tr}\left\{U_{x} U_{y}^{\dagger} U_{z} \mathcal{X}_{1 z x}^{\dagger}\right\}-\frac{1}{N_{c}}\left(\operatorname{Tr}\left\{U_{y}^{\dagger} U_{z}\right\}\left(\mathcal{H}_{1 x z}^{+}-\mathcal{H}_{1 z x}^{-}\right)+\operatorname{Tr}\left\{U_{y}^{\dagger} U_{x}\right\}\left(\mathcal{H}_{1 x z}^{-}-\mathcal{H}_{1 z x}^{+}\right)\right) \\
& \left.\left.+\frac{1}{N_{c}^{2}}\left(\operatorname{Tr}\left\{U_{z} U_{y}^{\dagger} U_{x} \mathcal{X}_{1 z x}^{\dagger}\right\}-\operatorname{Tr}\left\{U_{x} U_{y}^{\dagger} U_{z} \mathcal{X}_{1 x z}^{\dagger}\right\}\right)\right]\right\}
\end{aligned}
$$

In the flavor non-singlet case, because of the absence of mixing with operator $\mathcal{F}\left(x_{\perp}\right)$ the evolution equation for polarized structure functions is eq. (7.13). This might represent a great simplification to find its solution.

\section{Conclusions}

We have extended the high-energy OPE of the $T$-product of two electromagnetic currents at sub-eikonal level. The impact factors $\mathcal{I}_{1}^{\mu \nu}$ and $\mathcal{I}_{5}^{\mu \nu}$, given in eqs. (4.11), and (4.12) respectively, are part of the main results of this paper. They satisfy electromagnetic gauge invariance and conformal $\mathrm{SL}(2, C)$ invariance.

We have identified several new distribution functions, summarized in section 5 , which came up from the parametrization of the matrix elements of the operators associated to the impact factors $I_{1}^{\mu \nu}$ and $I_{5}^{\mu \nu}$. In the appendix $\mathrm{C}$ we have identified further distribution functions that, however, will not contribute to $g_{1}$ structure functions.

We found that the polarized and unpolarized quark distribution functions as well as the polarized gluon distributions are energy suppressed with respect to the unpolarized gluon ones. Moreover we have observed that the polarized gluon distributions $G_{L}$, and $G_{T}$ enter on the same footing with the polarized distribution functions $q_{5 L}, q_{5 T}, \tilde{q}_{5 L}, \tilde{q}_{5 T}$, because they are associated to the same impact factor $I_{5}^{\mu \nu}$. We also showed that under one loop evolution we have mixing also with the operators parametrized by the quark distribution functions $q_{1}, q_{1 T}, \tilde{q}_{1}, \tilde{q}_{1 T}$.

We have also observed the evolution equations are not in a closed form; after one loop we have generated new operators $\hat{\mathcal{X}}_{1 x y}, \hat{\mathcal{X}}_{5 x y}, \hat{\mathcal{H}}_{1 x y}^{+}, \hat{\mathcal{H}}_{5 x y}^{+}, \hat{\mathcal{H}}_{1 x y}^{-}, \hat{\mathcal{H}}_{5 x y}^{-}$.

In the evolution equations (see section 7 ), we have found also mixing between operators of different parity, i.e. operators $\hat{Q}_{5 x}, \hat{\tilde{Q}}_{5 x}, \hat{\mathcal{F}}_{x}, \hat{\mathcal{X}}_{5 x y}, \hat{\mathcal{H}}_{5 x y}^{+}$, and $\hat{\mathcal{H}}_{5 x y}^{-}$and operators $\hat{Q}_{1 x}$, $\hat{\tilde{Q}}_{1 x}, \hat{\mathcal{X}}_{1 x y}, \hat{\mathcal{H}}_{1 x y}^{+}$, and $\hat{\mathcal{H}}_{1 x y}^{-}$. These are all new evolution equations that are presented here for the first time. As already emphasized before, we are aware that the form of the evolution equations summarized in section 7 are not written in the usual form $\frac{d}{d \eta} \mathcal{O}^{\eta}=K \otimes \mathcal{O}^{\eta}$. The reason is that in all these equations there are contaminations of infrared divergences which remind us the double log of energy nature of the small-x resummation of quark polarized (and unpolarized) structure functions. We plan to disentangle the leading log from the double log of energy and to put the evolution equations of section 7 in a conventional form in future work where we will try to reproduce the result obtained in the double log formalism [36, 37]. 
We now compare our results with the ones obtained in recent literature $[7,8,56-58]$.

Let us start with the ones obtained in refs. $[7,8]$. In appendix $\mathrm{F}$ we have shown that, in the double logarithm approximation, the evolution equation for the operator $\operatorname{Tr}\left\{\left(\mathcal{F}_{x}+\right.\right.$ $\left.\left.\mathcal{Q}_{5 x}\right) U_{y}^{\dagger}\right\}$, eq. (F.13) (see also eq. (F.45) in the adjoint representation), does agree with the one derived in refs. $[7,8]$ provided that we neglect the mixing with operator $\hat{\mathcal{Q}}_{1 x}$. However, when we consider all diagrams we find some differences.

In refs. $[7,8]$ the evolution equations for the operators $\hat{Q}_{1 x}, \hat{Q}_{5 x}, \hat{\tilde{Q}}_{1 x}, \hat{\tilde{Q}}_{5 x}$ were not calculated. Only operator $\hat{\mathcal{Q}}_{5 x}$ was considered. With equation (7.9), we have shown that operator $\hat{\mathcal{F}}_{x}$ does mix with operators $\hat{Q}_{1 x}, \hat{Q}_{5 x}, \hat{\tilde{Q}}_{1 x}, \hat{\tilde{Q}}_{5 x}$, thus justifying the calculation of their evolution equations.

Here, for the first time, we calculated all the diagrams required for the evolution of the polarized (and unpolarized) structure functions including the quark-to-gluon propagator in the fundamental (see figures 12 and 13) and in the adjoint representation (see figure 15). The quark-to-gluon propagator diagrams have generated new operators, (6.59)(6.66), which will affect the spin dynamics at small-x.

In refs. $[7,8]$ the matrix element of operator $\operatorname{Tr}\left\{\hat{\mathcal{F}}_{x} \hat{U}_{y}^{\dagger}\right\}$ was associated only with the longitudinal helicity distributions. The same conclusion was reached also in ref. [58]. In section 5 (see also appendix $\mathrm{C}$ ), however, it was shown that the matrix element of operator $\operatorname{Tr}\left\{\hat{\mathcal{F}}_{x} \hat{U}_{y}^{\dagger}\right\}$ is, in general, parametrized with longitudinal and transverse distributions.

Sub-eikonal corrections in the framework of Color Glass Condensate (CGC) have been considered also in refs. $[56,57]$. At the moment the result in refs. $[56,57]$ is incomplete because sub-eikonal corrections due to pure transverse components of the field strength tensor, like $F_{i j}$, have not been included. As we have seen, such corrections turned out to be very important and central to the study of spin dynamics. Recently, the sub-eikonal corrections to the quark propagator have been considered in ref. [58]. The authors have reproduced the terms in operator $\hat{\mathcal{O}}_{1}$ of eq. (B.13), but their result seem to be missing some of the terms that are, instead, present in ref. [46] and that we presented here in eq. (3.1).

In ref. [9] the small-x behavior of the orbital angular momentum distributions was found as a generalization of the double log of energy for $g_{1}$ structure function originally found in refs. [36, 37]. On the other hand, in ref. [54] the same calculation was carried on in the frame work of saturation and Color Glass Condensate formalism. To our knowledge, it is not known whether the two results are consistent. Our work, among other things, set the foundation of the formalism that eventually will be able to reconcile the Double Logarithm Asymptotics formalism with the non-linear Wilson-line formalism.

Concluding, we obtained novel evolution equations for polarized structure functions (and unpolarized quark structure functions). New operators and new distribution functions have appeared for the first time in small-x physics. First, we have the appearance of the light-ray operators $\hat{Q}_{1 x}$ and $\hat{Q}_{5 x}$ multiplied by the usual dipole operator $\operatorname{Tr}\left\{\hat{U}_{x} \hat{U}_{y}^{\dagger}\right\}$; we have the operators $\operatorname{Tr}\left\{\hat{\tilde{Q}}_{1 x} U_{y}^{\dagger}\right\}$, and $\operatorname{Tr}\left\{\hat{\tilde{Q}}_{5 x} U_{y}^{\dagger}\right\}$; the gluon helicity and transverse distribution is obtained from $\operatorname{Tr}\left\{U_{z_{1}}^{\dagger} \mathcal{F}_{z_{2}}\right\}+\operatorname{Tr}\left\{U_{z_{1}} \mathcal{F}_{z_{2}}^{\dagger}\right\}$; and finally we have also the TMDs operators $\hat{\mathcal{X}}_{1 x y}, \hat{\mathcal{X}}_{5 x y}, \hat{\mathcal{H}}_{1 x y}^{+}, \hat{\mathcal{H}}_{5 x y}^{+}, \hat{\mathcal{H}}_{1 x y}^{-}, \hat{\mathcal{H}}_{5 x y}^{-}$. 


\section{Acknowledgments}

The author is grateful to I. Balitsky for numerous valuable discussions. He also thanks V.M. Braun for discussions and reading the manuscript, and Y. Kovchegov, and A. Vladimirov for discussions. This project has received funding from the European Union's Horizon 2020 research and innovation programme under grant No 824093.

\section{A Notation}

The notations used throughout this paper are the same as the ones used in ref. [46].

Given two light-cone vectors $p_{1}^{\mu}$ and $p_{2}^{\mu}$, with $p_{1}^{\mu} p_{2 \mu}=\frac{s}{2}$, we can decompose any coordinate as $x^{\mu}=\frac{2}{s} x_{*} p_{1}^{\mu}+\frac{2}{s} x_{\bullet} p_{2}^{\mu}+x_{\perp}^{\mu}$ with $x_{*}=x_{\mu} p_{2}^{\mu}=\sqrt{\frac{s}{2}} x^{+}, x_{\bullet}=x_{\mu} p_{1}^{\mu}=\sqrt{\frac{s}{2}} x^{-}$and $x^{ \pm}=\frac{x^{0} \pm x^{3}}{\sqrt{2}}$. Our metric is $(1,-1,-1,-1$,$) . We use the notation x_{\perp}^{\mu}=\left(0, x^{1}, x^{2}, 0\right)$ and $x^{i}=$ $\left(x^{1}, x^{2}\right)$ such that $x^{i} x_{i}=x_{\perp}^{\mu} x_{\mu}^{\perp}=-x_{\perp}^{2}$. So, Latin indexes assume values 1,2 , while Greek indexes run from 0 to 3 . We also use notation for scalar product $(x, y)_{\perp}=x^{1} y^{1}+x^{2} y^{2}$. For a vector in momentum space we have $k^{\mu}=\alpha p_{1}^{\mu}+\beta p_{2}^{\mu}+p_{\perp}^{\mu}$ with $\alpha=\sqrt{\frac{2}{s}} k^{+}$and $\beta=\sqrt{\frac{2}{s}} k^{-}$.

Under a longitudinal boost, the components of the gauge fields gets rescaled by the large boost parameter $\lambda$ as follows

$$
\begin{aligned}
& A_{\bullet}\left(x_{\bullet}, x_{*}, x_{\perp}\right) \rightarrow \lambda A_{\bullet}\left(\lambda^{-1} x_{\bullet}, \lambda x_{*}, x_{\perp}\right), \\
& A_{*}\left(x_{\bullet}, x_{*}, x_{\perp}\right) \rightarrow \lambda^{-1} A_{*}\left(\lambda^{-1} x_{\bullet}, \lambda x_{*}, x_{\perp}\right), \\
& A_{\perp}\left(x_{\bullet}, x_{*}, x_{\perp}\right) \rightarrow A_{\perp}\left(\lambda^{-1} x_{\bullet}, \lambda x_{*}, x_{\perp}\right) .
\end{aligned}
$$

while field strength tensor as

$$
\begin{aligned}
& F_{i \bullet}\left(x_{\bullet}, x_{*}, x_{\perp}\right) \rightarrow \lambda F_{i \bullet}\left(\lambda^{-1} x_{\bullet}, \lambda x_{*}, x_{\perp}\right), \\
& F_{i *}\left(x_{\bullet}, x_{*}, x_{\perp}\right) \rightarrow \lambda^{-1} F_{i *}\left(\lambda^{-1} x_{\bullet}, \lambda x_{*}, x_{\perp}\right), \\
& F_{\bullet *}\left(x_{\bullet}, x_{*}, x_{\perp}\right) \rightarrow F_{\bullet *}\left(\lambda^{-1} x_{\bullet}, \lambda x_{*}, x_{\perp}\right), \\
& F_{i j}\left(x_{\bullet}, x_{*}, x_{\perp}\right) \rightarrow F_{i j}\left(\lambda^{-1} x_{\bullet}, \lambda x_{*}, x_{\perp}\right) .
\end{aligned}
$$

Under the same large longitudinal boost the spinor fields get rescaled as

$$
\bar{\psi} t^{a} \not p_{1} \psi \rightarrow \lambda \bar{\psi} t^{a} \not p_{1} \psi, \quad \bar{\psi} t^{a} \gamma_{\nu}^{\perp} \psi \rightarrow \bar{\psi} t^{a} \gamma_{\nu}^{\perp} \psi, \quad \bar{\psi} t^{a} \not p_{2} \psi \rightarrow \lambda^{-1} \bar{\psi} t^{a} \not p_{2} \psi .
$$

In Schwinger representation, which will be frequently used throughout this paper, the free scalar propagator can be written as

$$
\left\langle x\left|\frac{i}{p^{2}+i \epsilon}\right| y\right\rangle=i \int d^{4} k \frac{e^{-i k \cdot(x-y)}}{k^{2}+i \epsilon},
$$

with $\langle k \mid x\rangle=e^{i x \cdot k}$. In (A.4) we used the $\hbar$-inspired notation $d^{4} k \equiv \frac{d^{4} k}{(2 \pi)^{4}}$ and $\delta^{(4)}(k)=$ $(2 \pi)^{4} \delta^{(4)}(k)$ so that, $\int d^{4} k \delta^{(4)}(k)=1$.

We define the gauge link at fixed transverse position as

$$
\left[u p_{1}, v p_{1}\right]_{z} \equiv\left[u p_{1}+z_{\perp}, v p_{1}+z_{\perp}\right] \equiv \operatorname{Pexp}\left\{i g \int_{v}^{u} d t A_{\bullet}\left(t p_{1}+z_{\perp}\right)\right\} .
$$


The derivative of the gauge link with respect to the transverse position is

$$
\begin{aligned}
\frac{\partial}{\partial z^{i}}\left[u p_{1}, v p_{1}\right]_{z}= & i g A_{i}\left(u p_{1}+z_{\perp}\right)\left[u p_{1}, v p_{1}\right]_{z}-i g\left[u p_{1}, v p_{1}\right]_{z} A_{i}\left(v p_{1}+z_{\perp}\right) \\
& -i g \int_{v}^{u} d s\left[u p_{1}, s p_{1}\right]_{z} F_{\bullet i}\left(p_{1} s+z_{\perp}\right)\left[p_{1} s, p_{1} v\right]_{z},
\end{aligned}
$$

with index $i=1,2$. From (A.6) we may formally define the transverse covariant derivative $\mathfrak{D}_{i}$ that acts on a non-local operator as

$$
\begin{aligned}
i \mathfrak{D}_{i}\left[u p_{1}, v p_{1}\right]_{z} & \equiv i \frac{\partial}{\partial z^{i}}\left[u p_{1}, v p_{1}\right]_{z}+g\left[A_{i}\left(z_{\perp}\right),\left[u p_{1}, v p_{1}\right]_{z}\right] \\
& =g \int_{v}^{u} d s\left[u p_{1}, s p_{1}\right]_{z} F_{\bullet i}\left(p_{1} s+z_{\perp}\right)\left[p_{1} s, p_{1} v\right]_{z},
\end{aligned}
$$

where we have used the implicit notation $\left[A_{i}\left(z_{\perp}\right),\left[u p_{1}, v p_{1}\right]_{z}\right]=A_{i}\left(z_{\perp}+u p_{1}\right)\left[u p_{1}, v p_{1}\right]_{z}-$ $\left[u p_{1}, v p_{1}\right]_{z} A_{i}\left(z_{\perp}+v p_{1}\right)$.

Given a gauge link $\left[x_{*}, y_{*}\right]_{z} \equiv\left[\frac{2}{s} x_{*} p_{1}+z_{\perp}, \frac{2}{s} y_{*} p_{1}+z_{\perp}\right]$, in Schwinger notation we have

$$
\left\langle x_{\perp}\left|\left[x_{*}, y_{*}\right]\right| y_{\perp}\right\rangle=\left[x_{*}, y_{*}\right]_{x} \delta^{(2)}(x-y) .
$$

The transverse momentum operator $\hat{P}_{i}=\hat{p}_{i}+g \hat{A}_{i}$ acts on the gauge link as

$$
\begin{aligned}
\left\langle x_{\perp}\left|\hat{P}_{i}\left[x_{*}, y_{*}\right]\right| y_{\perp}\right\rangle & \equiv\left\langle x_{\perp}\left|\left(\hat{p}_{i}+\hat{A}_{i}\left(x_{*}\right)\right)\left[x_{*}, y_{*}\right]\right| y_{\perp}\right\rangle \\
& =\left(i \frac{\partial}{\partial x^{i}}+A_{i}\left(x_{*}, x_{\perp}\right)\right)\left\langle x_{\perp}\left|\left[x_{*}, y_{*}\right]\right| y_{\perp}\right\rangle \\
\left\langle x_{\perp}\left|\left[x_{*}, y_{*}\right] \hat{P}_{i}\right| y_{\perp}\right\rangle & \equiv\left\langle x_{\perp}\left|\left[x_{*}, y_{*}\right]\left(\hat{p}_{i}+A_{i}\left(y_{*}\right)\right)\right| y_{\perp}\right\rangle \\
& =\left\langle x_{\perp}\left|\left[x_{*}, y_{*}\right]\right| y_{\perp}\right\rangle\left(-i \frac{\partial}{\partial y^{i}}+A_{i}\left(y_{*}, y_{\perp}\right)\right) .
\end{aligned}
$$

So, from (A.9), and (A.10), and using (A.7), and (A.8) we finally have

$$
\left\langle x_{\perp}\left|\left[\hat{P}_{i},\left[x_{*}, y_{*}\right]\right]\right| y_{\perp}\right\rangle=\left\langle x_{\perp}\left|i \mathfrak{D}_{i}\left[x_{*}, y_{*}\right]\right| y_{\perp}\right\rangle=\left\langle x_{\perp}\left|g \frac{2}{s} \int_{y_{*}}^{x_{*}} d \omega_{*}\left[x_{*}, \omega_{*}\right] F_{\bullet}\left[\omega_{*}, y_{*}\right]\right| y_{\perp}\right\rangle,
$$

where we used again the short-hand notation $\left[x_{*}, \omega_{*}\right] F_{i \bullet}\left[\omega_{*}, y_{*}\right]=\left[x_{*}, \omega_{*}\right] F_{i \bullet}\left(\omega_{*}\right)\left[\omega_{*}, y_{*}\right]$. From (A.11) we observe that the covariant derivative $i \mathfrak{D}_{i}$ acts on the gauge link even though the transverse coordinate has not been specified yet and, as matter of fact, it does not have to in order to know how it acts on the gauge link. Throughout the paper we will often use this property of the momentum operator $\hat{P}_{i}$ and the covariant derivative $i \mathfrak{D}_{i}$ without specifying the $b r a\left\langle x_{\perp}\right|$ and the ket $\left|y_{\perp}\right\rangle$.

\section{B Quark and gluon propagators with sub-eikonal corrections}

We want to extend the high-energy OPE to include sub-eikonal corrections in order to study polarized structure function at small-x. The idea is to proceed in a similar way as we did in the unpolarized case with the exception that now the quark propagator (and the gluon one) has also sub-eikonal corrections. In reference [46] a complete analysis of the quark and gluon propagator has been performed. All the sub-eikonal corrections (regardless of the twist) that scale as $\frac{1}{\lambda}$ with $\lambda$ a large boost parameter, have been identified.

In what follow we will summarize the all the propagator with sub-eikonal corrections. 


\section{B.1 Quark propagator in the background of gluon fields}

In this sub-section we are going to derive the quark propagator in the gluon background field. Here, however, we will perform a different expansion than the one we performed in ref. [46], but that was only suggested in the appendix of the same reference.

If we define $\mathcal{B}_{1}=\frac{4}{s^{2}} F_{\bullet} \sigma_{* \bullet}+\frac{1}{2} \sigma_{i j} F^{i j}$, and $O=\left\{p_{\perp}^{\mu}, A_{\mu}^{\perp}\right\}+\left\{\frac{2}{s} P_{\bullet}, A_{*}\right\}-g A_{\perp}^{2}$, then the quark propagator up to sub-eikonal corrections is

$$
\begin{aligned}
\langle x| & \not P \frac{i}{P^{2}+\frac{g}{2} F_{\mu \nu} \sigma^{\mu \nu}}|y\rangle \\
= & i \not D\langle x|\left[\frac{i}{p^{2}+2 g \alpha A_{\bullet}+g O}-\frac{i}{p^{2}+2 g \alpha A_{\bullet}+g O} i g \frac{2}{s} F_{i \bullet} \gamma^{i} \not p_{2} \frac{1}{p^{2}+2 g \alpha A_{\bullet}+g O}\right. \\
& -\frac{i}{p^{2}+2 g \alpha A_{\bullet}} g \mathcal{B}_{1} \frac{1}{p^{2}+2 g \alpha A_{\bullet}}+\frac{i}{p^{2}+2 g \alpha A_{\bullet}} i g \frac{2}{s} F_{i \bullet} \gamma^{i} \not p_{2} \frac{1}{p^{2}+2 g \alpha A_{\bullet}} g \mathcal{B}_{1} \frac{1}{p^{2}+2 g \alpha A_{\bullet}} \\
& \left.+\frac{i}{p^{2}+2 g \alpha A_{\bullet}} g \mathcal{B}_{1} \frac{1}{p^{2}+2 g \alpha A_{\bullet}} i g \frac{2}{s} F_{i \bullet} \gamma^{i} \not p_{2} \frac{1}{p^{2}+2 g \alpha A_{\bullet}}\right]|y\rangle .
\end{aligned}
$$

Now we need the scalar propagator up to sub-eikonal corrections [46]

$$
\begin{aligned}
& \left\langle x\left|\frac{i}{P^{2}+i \epsilon}\right| y\right\rangle=\left\langle x\left|\frac{i}{p^{2}+2 \alpha g A \bullet+g O+i \epsilon}\right| y\right\rangle \\
& =\left[\int_{0}^{+\infty} \frac{d \alpha}{2 \alpha} \theta\left(x_{*}-y_{*}\right)-\int_{-\infty}^{0} \frac{d \alpha}{2 \alpha} \theta\left(y_{*}-x_{*}\right)\right] e^{-i \alpha\left(x_{\bullet}-y_{\bullet}\right)} \\
& \quad \times\left\langle x_{\perp}\right| e^{-i \frac{\hat{p}_{\perp}^{2}}{\alpha s} x_{*}}\left\{\left[x_{*}, y_{*}\right]+\frac{i g}{2 \alpha}\left[\frac{2}{s} x_{*}\left(\left\{P_{i}, A^{i}\left(x_{*}\right)\right\}-g A_{i}\left(x_{*}\right) A^{i}\left(x_{*}\right)\right)\left[x_{*}, y_{*}\right]\right.\right. \\
& \quad-\left[x_{*}, y_{*}\right] \frac{2}{s} y_{*}\left(\left\{P_{i}, A^{i}\left(y_{*}\right)\right\}-g A_{i}\left(y_{*}\right) A^{i}\left(y_{*}\right)\right)+\int_{y_{*}}^{x_{*}} d \frac{2}{s} \omega_{*}\left(\left\{P^{i},\left[x_{*}, \omega_{*}\right] \frac{2}{s} \omega_{*} F_{i \bullet}\left(\omega_{*}\right)\left[\omega_{*}, y_{*}\right]\right\}\right. \\
& \left.\left.\left.\quad+g \int_{\omega_{*}}^{x_{*}} d \frac{2}{s} \omega_{*}^{\prime} \frac{2}{s}\left(\omega_{*}-\omega_{*}^{\prime}\right)\left[x_{*}, \omega_{*}^{\prime}\right] F_{\bullet}^{i}\left[\omega_{*}^{\prime}, \omega_{*}\right] F_{i \bullet}\left[\omega_{*}, y_{*}\right]\right)\right]\right\} e^{i \frac{\hat{p}_{\perp}^{2}}{\alpha s} y_{*}\left|y_{\perp}\right\rangle . \quad(\mathrm{B} .2)}
\end{aligned}
$$

Now we observe that

$$
\begin{aligned}
& i \not D\left(e^{-i \alpha\left(x_{\bullet}-y_{\bullet}\right)}\left\langle x_{\perp}\left|e^{-i \frac{\hat{p}_{\perp}^{2}}{\alpha s} x_{*}}\left[x_{*}, y_{*}\right]\right| z_{\perp}\right\rangle\right) \\
= & \left(i \not x^{x}+g \frac{2}{s} \not_{2} A_{\bullet}\left(x_{*}, x_{\perp}\right)+g A_{\perp}\left(x_{*}, x_{\perp}\right)\right) e^{-i \alpha\left(x_{\bullet}-y_{\bullet}\right)}\left\langle x_{\perp}\left|e^{-i \frac{\hat{p}_{\perp}^{2}}{\alpha s} x_{*}}\left[x_{*}, y_{*}\right]\right| z_{\perp}\right\rangle \\
= & e^{-i \alpha\left(x_{\bullet}-y_{\bullet}\right)}\left\langle x_{\perp}\right| e^{-i \frac{\hat{p}_{\perp}^{2}}{\alpha s} x_{*}} \\
& \times\left(\frac{1}{\alpha s} \hat{p h} p_{2} \hat{p p}+i \frac{2}{s} \not p_{2} D_{\bullet}^{x}+\frac{i x_{*}}{\alpha s}\left[\hat{p}_{\perp}^{2}, g \frac{2}{s} \not p_{2} A_{\bullet}\left(x_{*}\right)\right]+g A_{\perp}\left(x_{*}\right)\right)\left[x_{*}, y_{*}\right]\left|z_{\perp}\right\rangle .
\end{aligned}
$$

Note that $i\left[p_{\perp}^{2}, g A_{\bullet}\left(x_{*}\right)\right]=g\left\{p^{i}, F_{i \bullet}\left(x_{*}\right)+D_{\bullet} A_{i}\left(x_{*}\right)\right\}$. The field strength tensor $F_{i \bullet}\left(x_{*}\right)=0$ since $x_{*}$ is outside the shock-wave $\left(x_{*}\right.$ and $y_{*}$ here are always point outside the shock-wave which will be sent to $+\infty$ and $-\infty$ respectively). Similarly, we can set all the transverse fields at the edges of the gauge-link (outside the shock-wave) to zero since they are pure 
gauge. Moreover, we make use of $i D_{\bullet}\left[x_{*}, y_{*}\right]_{x}=\left(i \partial_{\bullet}^{x}+g A_{\bullet}\left(x_{*}\right)\right)\left[x_{*}, y_{*}\right]_{x}=0$. So, we may reduce (B.3) to

$$
\begin{gathered}
\left(i \not \partial^{x}+g \frac{2}{s} \not p_{2} A_{\bullet}\left(x_{*}, x_{\perp}\right)+g A_{\perp}\left(x_{*}, x_{\perp}\right)\right) e^{-i \alpha\left(x_{\bullet}-y_{\bullet}\right)}\left\langle x_{\perp}\left|e^{-i \frac{\hat{p}_{\perp}^{2}}{\alpha s} x_{*}}\right| z_{\perp}\right\rangle \\
=e^{-i \alpha\left(x_{\bullet}-y_{\bullet}\right)}\left\langle x_{\perp}\left|e^{-i \frac{\hat{p}_{\perp}^{2}}{\alpha s} x_{*}} \frac{1}{\alpha s} \not \not p p_{2} \not p\left[x_{*}, y_{*}\right]\right| z_{\perp}\right\rangle .
\end{gathered}
$$

We will also need the following two identities. Given two generic operators $\mathcal{A}_{1}$ and $\mathcal{A}_{2}$, we have

$$
\begin{aligned}
& \left\langle x\left|\frac{1}{p^{2}+2 g \alpha A_{\bullet}+i \epsilon} A_{1} \frac{1}{p^{2}+2 g \alpha A_{\bullet}+i \epsilon}\right| y\right\rangle \\
& =\left[-\int_{0}^{+\infty} \frac{d \alpha}{4 \alpha^{2}} \theta\left(x_{*}-y_{*}\right)+\int_{-\infty}^{0} \frac{d \alpha}{4 \alpha^{2}} \theta\left(y_{*}-x_{*}\right)\right] e^{-i \alpha\left(x_{\bullet}-y_{\bullet}\right)} \\
& \times \int_{y_{*}}^{x_{*}} d \frac{2}{s} z_{1 *}\left\langle x_{\perp}\left|e^{-i \frac{\hat{p}_{\perp}^{2}}{\alpha s} x_{*}}\left[x_{*}, z_{1 *}\right] A_{1}\left[z_{1 *}, y_{*}\right] e^{i \frac{\hat{p}_{\perp}^{2}}{\alpha s} y_{*}}\right| y_{\perp}\right\rangle
\end{aligned}
$$

and

$$
\begin{aligned}
& \left\langle x\left|\frac{1}{p^{2}+2 g \alpha A_{\bullet}+i \epsilon} A_{1} \frac{1}{p^{2}+2 g \alpha A_{\bullet}+i \epsilon} A_{2} \frac{1}{p^{2}+2 g \alpha A_{\bullet}+i \epsilon}\right| y\right\rangle \\
& =\left[i \int_{0}^{+\infty} \frac{d \alpha}{8 \alpha^{3}} \theta\left(x_{*}-y_{*}\right)-i \int_{-\infty}^{0} \frac{d \alpha}{8 \alpha^{3}} \theta\left(y_{*}-x_{*}\right)\right] e^{-i \alpha\left(x_{\bullet}-y_{\bullet}\right)} \\
& \quad \times \int_{y_{*}}^{x_{*}} d \frac{2}{s} z_{2 *} \int_{z_{2 *}}^{x_{*}} d \frac{2}{s} z_{1 *}\left\langle x_{\perp}\left|e^{-i \frac{\hat{p}_{\perp}^{2}}{\alpha s} x_{*}}\left[x_{*}, z_{1 *}\right] A_{1}\left[z_{1 *}, z_{2 *}\right] A_{2}\left[z_{2 *}, y_{*}\right] e^{i \frac{\hat{p}_{\perp}^{2}}{\alpha s} y_{*}}\right| y_{\perp}\right\rangle
\end{aligned}
$$

To obtain (B.5) and (B.6), one hae to insert a complite set of states between the operators $A_{1}$ and $A_{2}$ and use the eikonal term of the scalar propagator in eq. (B.2). Another identities that we are going to need is

$$
\begin{aligned}
& \frac{i z_{*}}{\alpha s}\left[p_{\perp}^{2}, F_{j \bullet}\right]+\frac{2}{s} z_{*} \frac{i g}{2 \alpha}\left(-\left\{P_{i}, A^{i}\right\}+g A_{i} A^{i}\right) F_{j \bullet}+F_{j \bullet} \frac{2}{s} z_{*} \frac{i g}{2 \alpha}\left(\left\{P_{i}, A^{i}\right\}-g A_{i} A^{i}\right) \\
& \quad=-\frac{i z_{*}}{\alpha s}\left\{P^{i}, i D_{i} F_{j \bullet}\right\}
\end{aligned}
$$

So, using eq. (B.2), and identities (B.3), (B.4), (B.5), (B.6), and (B.7), the first two terms of expansion (B.1) become

$$
\begin{aligned}
& i \not D\left\langle x\left|\frac{i}{p^{2}+2 g \alpha A_{\bullet}+g O}-\frac{i}{p^{2}+2 g \alpha A_{\bullet}+g O} i g \frac{2}{s} F_{i \bullet} \gamma^{i} \not p_{2} \frac{1}{p^{2}+2 g \alpha A_{\bullet}+g O}\right| y\right\rangle \\
& =\left[\int_{0}^{+\infty} \frac{d \alpha}{2 \alpha} \theta\left(x_{*}-y_{*}\right)-\int_{-\infty}^{0} \frac{d \alpha}{2 \alpha} \theta\left(y_{*}-x_{*}\right)\right] e^{-i \alpha\left(x_{\bullet}-y_{\bullet}\right)} \frac{1}{\alpha s}\left\langle x_{\perp}\right| e^{-i \frac{\hat{p}_{\perp}^{2}}{\alpha s} x_{*}} \not p p_{2}\left[x_{*}, y_{*}\right] \not p \\
& +\frac{i g}{2 \alpha} \int_{y_{*}}^{x_{*}} d \frac{2}{s} \omega_{*} \not p p_{2}\left(\frac{2}{s} \omega_{*}\left\{P_{i},\left[x_{*}, \omega_{*}\right] F^{i} \cdot\left[\omega_{*}, y_{*}\right]\right\}\right. \\
& \left.\left.+g \int_{\omega_{*}}^{x_{*}} d \frac{2}{s} \omega_{*}^{\prime} \frac{2}{s}\left(\omega_{*}-\omega_{*}^{\prime}\right)\left[x_{*}, \omega_{*}^{\prime}\right] F^{i} \bullet\left[\omega_{*}^{\prime}, \omega_{*}\right] F_{i \bullet}\left[\omega_{*}, y_{*}\right]\right) \not p\right\} e^{i \frac{\hat{p}_{\perp}^{2}}{\alpha s} y_{*}}\left|y_{\perp}\right\rangle
\end{aligned}
$$


Note that to get eq. (B.8) we have pushed the operator $\hat{p}$ all the way to the right so to have a $\hat{p}$ to the left and another one to the right. This is the structure of the quark propagator in the background of shock-wave: free propagator until the interaction with the scock-wave, eikonal plus sub-eikonal interaction with the shock-wave, and again free propagation after the shock-wave. Note also that, since the operator $\hat{\not p}$ is outside the shock-wave i.e. is at the point $x_{*}$, it can be promoted to $\hat{P}$ because the gauge fields are pure gauge outside the shock-wave (points $x_{*}$ and $y_{*}$ ). Then, to push the operator $\hat{P}$ to the right we repeatedly use

$$
\begin{aligned}
& \left\langle x_{\perp}\left|\not \not p p_{2} \not P\left[x_{*}, y_{*}\right]\right| y_{\perp}\right\rangle=\left\langle x_{\perp}\left|\not \not p p_{2}\left(\alpha \not p_{1}+\not P_{\perp}\right)\left[x_{*}, y_{*}\right]\right| y_{\perp}\right\rangle \\
& =\left\langle x_{\perp}\left|\not \not p p_{2}\left(\gamma^{i} i \mathfrak{D}_{i}\left[x_{*}, y_{*}\right]+\left[x_{*}, y_{*}\right]\left(\alpha \not p_{1}+\not P_{\perp}\right)\right)\right| y_{\perp}\right\rangle
\end{aligned}
$$

and the definition of $\mathfrak{D}_{i}$ eq. (A.7). Recall also that $\alpha=\frac{2}{s} p_{*}=\frac{2}{s} i \frac{\partial}{\partial x_{\bullet}}$, and it commutes with all the fields because they do not depend on $x_{\bullet}$. In a very similar way, we have

$$
\begin{aligned}
& i \not D\langle x|\left[-\frac{i}{p^{2}+2 g \alpha A_{\bullet}} g \frac{1}{2} \sigma_{i j} F^{i j} \frac{1}{p^{2}+2 g \alpha A_{\bullet}}\right. \\
& +\frac{i}{p^{2}+2 g \alpha A_{\bullet}} i g \frac{2}{s} F_{i \bullet} \gamma^{i} \not p_{2} \frac{1}{p^{2}+2 g \alpha A_{\bullet}} \frac{1}{2} \sigma_{i j} F^{i j} \frac{1}{p^{2}+2 g \alpha A_{\bullet}} \\
& \left.+\frac{i}{p^{2}+2 g \alpha A_{\bullet}} \frac{1}{2} \sigma_{i j} F^{i j} \frac{1}{p^{2}+2 g \alpha A_{\bullet}} i g \frac{2}{s} F_{i \bullet} \gamma^{i} \not p_{2} \frac{1}{p^{2}+2 g \alpha A_{\bullet}}\right]|y\rangle \\
& =\left[\int_{0}^{+\infty} \frac{d \alpha}{2 \alpha} \theta\left(x_{*}-y_{*}\right)-\int_{-\infty}^{0} \frac{d \alpha}{2 \alpha} \theta\left(y_{*}-x_{*}\right)\right] e^{-i \alpha\left(x_{\bullet}-y_{\bullet}\right)} \frac{1}{\alpha s}\left\langle x_{\perp}\right| e^{-i \frac{\hat{p}_{\perp}^{2}}{\alpha s} x_{*}} \\
& \quad \times\left[\frac { i g } { 2 \alpha } \int _ { y _ { * } } ^ { x _ { * } } d \frac { 2 } { s } z _ { * } \not p p p _ { 2 } \left(\left[x_{*}, z_{*}\right] \frac{1}{2} F_{i j} \sigma^{i j}\left[z_{*}, y_{*}\right] \not p+\left\{p^{k},\left[x_{*}, z_{*}\right] i F_{k j} \gamma^{j}\left[z_{*}, y_{*}\right]\right\}\right.\right. \\
& \left.\left.\quad+\left[x_{*}, z_{*}\right] i F_{k j} \gamma^{j}\left(i D^{k}\left[z_{*}, y_{*}\right]\right)-\left(i D^{k}\left[x_{*}, z_{*}\right]\right) i F_{k j} \gamma^{j}\left[z_{*}, y_{*}\right]\right)\right] e^{i \frac{\hat{p}_{\perp}^{2}}{\alpha s} y_{*}\left|y_{\perp}\right\rangle}
\end{aligned}
$$

and

$$
\begin{aligned}
& i \not D\langle x|\left[-\frac{i}{p^{2}+2 g \alpha A_{\bullet}} g \frac{4}{s^{2}} F_{\bullet *} \sigma_{* \bullet} \frac{1}{p^{2}+2 g \alpha A_{\bullet}}\right. \\
& +\frac{i}{p^{2}+2 g \alpha A_{\bullet}} i g \frac{2}{s} F_{i \bullet} \gamma^{i} \not p_{2} \frac{1}{p^{2}+2 g \alpha A_{\bullet}} \frac{4}{s^{2}} F_{\bullet *} \sigma_{* \bullet} \frac{1}{p^{2}+2 g \alpha A_{\bullet}} \\
& \left.+\frac{i}{p^{2}+2 g \alpha A_{\bullet}} \frac{4}{s^{2}} F_{\bullet *} \sigma_{* \bullet} \frac{1}{p^{2}+2 g \alpha A_{\bullet}} i g \frac{2}{s} F_{\bullet} \gamma^{i} \not p_{2} \frac{1}{p^{2}+2 g \alpha A_{\bullet}}\right]|y\rangle \\
& =\left[\int_{0}^{+\infty} \frac{\pi \alpha}{2 \alpha} \theta\left(x_{*}-y_{*}\right)-\int_{-\infty}^{0} \frac{d \alpha}{2 \alpha} \theta\left(y_{*}-x_{*}\right)\right] e^{-i \alpha\left(x_{\bullet}-y_{\bullet}\right)} \frac{1}{\alpha s}\left\langle x_{\perp}\right| e^{-i \frac{\hat{p}_{\perp}^{2}}{\alpha s} x_{*}} \\
& \quad \times\left[\frac{i g}{2 \alpha} \int_{y_{*}}^{x_{*}} d \frac{2}{s} z_{*} \not p p_{2}\left(\alpha \not p_{1}-\not p_{\perp}\right)\left[x_{*}, z_{*}\right] i \frac{2}{s} F_{\bullet *}\left[z_{*}, y_{*}\right]+\frac{i g}{2 \alpha} \int_{y_{*}}^{x_{*}} d \frac{2}{s} z_{*}\right. \\
& \left.\quad \times \not p p_{2}\left(\left(i \not D_{\perp}\left[x_{*}, z_{*}\right]\right) i \frac{2}{s} F_{\bullet *}\left[z_{*}, y_{*}\right]-\left[x_{*}, z_{*}\right] i \frac{2}{s} F_{\bullet *}\left(i \not D_{\perp}\left[z_{*}, y_{*}\right]\right)\right)\right] e^{i \frac{\hat{p}_{\perp}^{2}}{\alpha s} y_{*}\left|y_{\perp}\right\rangle}
\end{aligned}
$$

Summing the three terms (B.8), (B.10), and (B.11) and symmetrizing the propagator by adding 1.h.s. of eq. (B.1) with $\not P$ to the right, we arrive at the quark propagator with 
sub-eikonal corrections in the background of gluon fields [46]

$$
\begin{aligned}
\left\langle x\left|\frac{i}{\hat{p}+i \epsilon}\right| y\right\rangle= & {\left[\int_{0}^{+\infty} \frac{d \alpha}{2 \alpha} \theta\left(x_{*}-y_{*}\right)-\int_{-\infty}^{0} \frac{d \alpha}{2 \alpha} \theta\left(y_{*}-x_{*}\right)\right] e^{-i \alpha\left(x_{\bullet}-y_{\bullet}\right)} \frac{1}{\alpha s} } \\
& \times\left\langle x_{\perp}\right| e^{-i \frac{\hat{p}_{\perp}^{2}}{\alpha s} x_{*}}\left\{\hat{p}_{p} p_{2}\left[x_{*}, y_{*}\right] \hat{p}+\hat{p} \not p_{2} \hat{\mathcal{O}}_{1}\left(p_{\perp} ; x_{*}, y_{*}\right) \hat{\not p}\right. \\
& \left.+\hat{p} \not p_{2} \frac{1}{2} \hat{\mathcal{O}}_{2}\left(p_{\perp} ; x_{*}, y_{*}\right)-\frac{1}{2} \hat{\mathcal{O}}_{2}\left(x_{*}, y_{*} ; p_{\perp}\right) \not p_{2} \not \hat{p}\right\} e^{i \frac{\hat{p}_{\perp}^{2}}{\alpha s} y_{*}}\left|y_{\perp}\right\rangle+O\left(\lambda^{-2}\right) .
\end{aligned}
$$

with

$$
\begin{aligned}
& \hat{\mathcal{O}}_{1}\left(x_{*}, y_{*} ; p_{\perp}\right) \\
& \begin{aligned}
\frac{i g}{2 \alpha} \int_{y_{*}}^{x_{*}} d \frac{2}{s} \omega_{*}( & {\left[x_{*}, \omega_{*}\right] \frac{1}{2} \sigma^{i j} F_{i j}\left[\omega_{*}, y_{*}\right]+\left\{\hat{p}^{i},\left[x_{*}, \omega_{*}\right] \frac{2}{s} \omega_{*} F_{i \bullet}\left(\omega_{*}\right)\left[\omega_{*}, y_{*}\right]\right\} } \\
& \left.+g \int_{\omega_{*}}^{x_{*}} d \frac{2}{s} \omega_{*}^{\prime} \frac{2}{s}\left(\omega_{*}-\omega_{*}^{\prime}\right)\left[x_{*}, \omega_{*}^{\prime}\right] F^{i} \cdot\left[\omega_{*}^{\prime}, \omega_{*}\right] F_{i \bullet}\left[\omega_{*}, y_{*}\right]\right),
\end{aligned}
\end{aligned}
$$

and

$$
\begin{aligned}
& \hat{\mathcal{O}}_{2}\left(p_{\perp} ; x_{*}, y_{*}\right) \\
& =\frac{i g}{2 \alpha} \int_{y_{*}}^{x_{*}} d \frac{2}{s} \omega_{*}\left[\left\{\hat{p}^{k},\left[x_{*}, \omega_{*}\right] i F_{k j} \gamma^{j}\left[\omega_{*}, y_{*}\right]\right\}+\left(\alpha \not p_{1}-\hat{p}_{\perp}\right)\left[x_{*}, \omega_{*}\right] i \frac{2}{s} F_{\bullet *}\left[\omega_{*}, y_{*}\right]\right. \\
& \quad+\int_{\omega_{*}}^{x_{*}} d \frac{2}{s} \omega_{*}^{\prime}\left(\left[x_{*}, \omega_{*}^{\prime}\right] g F^{k}\left[\omega_{*}^{\prime}, \omega_{*}\right] i F_{k j} \gamma^{j}\left[\omega_{*}, y_{*}\right]-\left[x_{*}, \omega_{*}^{\prime}\right] i F_{k j} \gamma^{j}\left[\omega_{*}^{\prime}, \omega_{*}\right] g F_{\bullet}^{k}\left[\omega_{*}, y_{*}\right]\right. \\
& \left.\left.\quad+\left[x_{*}, \omega_{*}^{\prime}\right] i \frac{2}{s} F_{\bullet *}\left[\omega_{*}^{\prime}, \omega_{*}\right] \gamma^{k} g F_{k \bullet}\left[\omega_{*}, y_{*}\right]-\left[x_{*}, \omega_{*}^{\prime}\right] \gamma^{k} g F_{k \bullet}\left[\omega_{*}^{\prime}, \omega_{*}\right] i \frac{2}{s} F_{\bullet *}\left[\omega_{*}, y_{*}\right]\right)\right] .
\end{aligned}
$$

where $\left\{\hat{p}^{i},\left[x_{*}, \omega_{*}\right]\right\}=\hat{p}^{i}\left[x_{*}, \omega_{*}\right]+\left[x_{*}, \omega_{*}\right] \hat{p}^{i}$.

The definition of operator $\hat{\mathcal{O}}_{2}$ given here differs from the one given in the previous work, ref. [46], because using the identity $\left\{\gamma^{k}, \gamma^{i} \gamma^{j}\right\}=2 \gamma^{k} g^{i j}$ the term $\frac{i}{4}\left\{\left(i \not D_{\perp} F_{i j}\right), \gamma^{i} \gamma^{j}\right\}$ is identically zero.

Now let us define the operator $\mathcal{O}_{j}$ as follow

$$
\begin{aligned}
& \hat{\mathcal{O}}_{j}\left(p_{\perp} ; x_{*}, y_{*}\right) \\
& \equiv \frac{i g}{2 \alpha} \int_{y_{*}}^{x_{*}} d \frac{2}{s} \omega_{*}\left[\left\{\hat{p}^{k},\left[x_{*}, \omega_{*}\right] i F_{k j}\left[\omega_{*}, y_{*}\right]\right\}\right. \\
& \quad+\int_{\omega_{*}}^{x_{*}} d \frac{2}{s} \omega_{*}^{\prime}\left(\left[x_{*}, \omega_{*}^{\prime}\right] g F_{\bullet}^{k}\left[\omega_{*}^{\prime}, \omega_{*}\right] i F_{k j}\left[\omega_{*}, y_{*}\right]-\left[x_{*}, \omega_{*}^{\prime}\right] i F_{k j}\left[\omega_{*}^{\prime}, \omega_{*}\right] g F_{\bullet}^{k}\left[\omega_{*}, y_{*}\right]\right. \\
& \left.\left.\quad+\left[x_{*}, \omega_{*}^{\prime}\right] i \frac{2}{s} F_{\bullet *}\left[\omega_{*}^{\prime}, \omega_{*}\right] g F_{j}\left[\omega_{*}, y_{*}\right]-\left[x_{*}, \omega_{*}^{\prime}\right] g F_{j \bullet}\left[\omega_{*}^{\prime}, \omega_{*}\right] i \frac{2}{s} F_{\bullet *}\left[\omega_{*}, y_{*}\right]\right)\right] .
\end{aligned}
$$

and we can write

$$
\hat{p p p}_{2} \gamma^{j} \hat{\mathcal{O}}_{j}-\hat{\mathcal{O}}_{j} \gamma^{j} \not p_{2} \hat{p}=i \alpha s \epsilon^{i j} \gamma^{5} \gamma_{i} \hat{\mathcal{O}}_{j}-\not p_{2}\left[\hat{p}^{j}, \hat{\mathcal{O}}_{j}\right]-i \epsilon^{i j} \gamma^{5} \not p_{2}\left\{\hat{p}_{i}, \hat{\mathcal{O}}_{j}\right\}
$$

$\left(\right.$ recall $\left.\not p \not p_{2}=\alpha \not p_{1} \not p_{2}+\not p_{\perp} \not p_{2}\right)$. 
Moreover, we may define ${ }^{1}$

$$
\hat{\mathcal{O}}_{\bullet *}\left(x_{*}, y_{*}\right) \equiv \frac{i g}{2 \alpha} \int_{y_{*}}^{x_{*}} d \frac{2}{s} \omega_{*}\left[x_{*}, \omega_{*}\right] i \frac{2}{s} F_{\bullet *}\left[\omega_{*}, y_{*}\right]
$$

so we may write

$$
\hat{\not p} p_{2}\left(\alpha \not p_{1}-\hat{p}_{\perp}\right) \hat{\mathcal{O}}_{\bullet *}-\hat{\mathcal{O}}_{\bullet *}\left(\alpha \not p_{1}-\hat{p}_{\perp}\right) \not p_{2} \hat{\not p}=i \alpha s \gamma^{5} \gamma_{j} \epsilon^{i j}\left\{p_{i}, \mathcal{O}_{\bullet *}\right\}-\not p_{2}\left[\hat{p}_{\perp}^{2}, \hat{\mathcal{O}}_{\bullet *}\right]
$$

Using the operators $\mathcal{O}_{j}\left(x_{*}, y_{*}\right)$ and $\mathcal{O}_{\bullet}\left(x_{*}, y_{*}\right)$ the quark propagator (B.12) becomes eq. (3.1).

\section{B.2 Gluon propagator in the background of gluon field}

The gluon propagator with sub-eikonal corrections in the background of gluon fields is [46]

$$
\begin{aligned}
\left\langle A_{\mu}^{a}(x) A_{\nu}^{b}(y)\right\rangle_{A} \\
=\left[-\int_{0}^{+\infty} \frac{d \alpha}{2 \alpha} \theta\left(x_{*}-y_{*}\right)+\int_{-\infty}^{0} \frac{d \alpha}{2 \alpha} \theta\left(y_{*}-x_{*}\right)\right] e^{-i \alpha\left(x_{\bullet}-y_{\bullet}\right)}\left\langle x_{\perp}\right| e^{-i \frac{\hat{p}_{\perp}^{2}}{\alpha s} x_{*}} \\
\quad \times\left(\delta_{\mu}^{\xi}-\frac{2}{s} \frac{p_{2 \mu} p^{\xi}}{\alpha}\right) \mathcal{O}_{\alpha}\left(x_{*}, y_{*}\right)\left(g_{\xi \nu}-\frac{2}{s} \frac{p_{\xi} p_{2 \nu}}{\alpha}\right) e^{i \frac{\hat{p}_{\perp}^{2}}{\alpha s} y_{*}}\left|y_{\perp}\right\rangle^{a b}+i\left\langle x\left|\frac{4}{s^{2}} \frac{p_{2 \mu} p_{2 \nu}}{\alpha^{2}}\right| y\right\rangle^{a b} \\
\quad+\left[-\int_{0}^{+\infty} \frac{d \alpha}{2 \alpha} \theta\left(x_{*}-y_{*}\right)+\int_{-\infty}^{0} \frac{d \alpha}{2 \alpha} \theta\left(y_{*}-x_{*}\right)\right] e^{-i \alpha\left(x_{\bullet}-y_{\bullet}\right)}\left\langle x_{\perp}\right| e^{-i \frac{\hat{p}_{\perp}^{2}}{\alpha s} x_{*}} \\
\quad \times\left[\mathfrak{G}_{1 \mu \nu}^{a b}\left(x_{*}, y_{*} ; p_{\perp}\right)+\mathfrak{G}_{2 \mu \nu}^{a b}\left(x_{*}, y_{*} ; p_{\perp}\right)+\mathfrak{G}_{3 \mu \nu}^{a b}\left(x_{*}, y_{*} ; p_{\perp}\right)+\mathfrak{G}_{4 \mu \nu}^{a b}\left(x_{*}, y_{*} ; p_{\perp}\right)\right] \\
\quad \times e^{i \frac{\hat{p}_{\perp}^{2}}{\alpha s} y_{*}}\left|y_{\perp}\right\rangle+O\left(\lambda^{-2}\right),
\end{aligned}
$$

where we defined

$$
\begin{aligned}
\mathcal{O}_{\alpha}\left(x_{*}, y_{*}\right) \equiv\left[x_{*}, y_{*}\right]+\frac{i g}{2 \alpha} \int_{y_{*}}^{x_{*}} d \frac{2}{s} \omega_{*}\left(\left\{p^{i},\left[x_{*}, \omega_{*}\right] \frac{2}{s} \omega_{*} F_{i \bullet}\left(\omega_{*}\right)\left[\omega_{*}, y_{*}\right]\right\}\right. \\
\left.+g \int_{\omega_{*}}^{x_{*}} d \frac{2}{s} \omega_{*}^{\prime} \frac{2}{s}\left(\omega_{*}-\omega_{*}^{\prime}\right)\left[x_{*}, \omega_{*}^{\prime}\right] F^{i} \bullet\left[\omega_{*}^{\prime}, \omega_{*}\right] F_{i \bullet}\left[\omega_{*}, y_{*}\right]\right) .
\end{aligned}
$$

${ }^{1}$ Note that the term of eq. (B.17), since fields do not depend on $x_{\bullet}$, may be analyzed similarly to the term $\left\{P_{\bullet}, A_{*}\right\}$ in appendix $\mathrm{C}$ of ref. [46]. The result is that it may be reduced to gauge dependent terms $A_{*}\left[x_{*}, y_{*}\right]-\left[x_{*}, y_{*}\right] A_{*}$. 
and

$$
\begin{aligned}
\mathfrak{G}_{1 \mu \nu}^{a b}\left(x_{*}, y_{*} ; p_{\perp}\right)= & -\frac{g p_{2 \mu} p_{2 \nu}}{s^{2} \alpha^{3}} \int_{y_{*}}^{x_{*}} d \frac{2}{s} \omega_{*}\left[4 p^{i}\left[x_{*}, \omega_{*}\right] F_{i j}\left[\omega_{*}, y_{*}\right] p^{j}\right. \\
& \left.+i g \int_{\omega_{*}^{\prime}}^{x_{*}} d \frac{2}{s} \omega_{*}^{\prime} \frac{2}{s}\left(\omega_{*}^{\prime}-\omega_{*}\right)\left[x_{*}, \omega_{*}^{\prime}\right] i D^{i} F_{i \bullet}\left[\omega_{*}^{\prime}, \omega_{*}\right] i D^{j} F_{j \bullet}\left[\omega_{*}, y_{*}\right]\right]^{a b} \\
\mathfrak{G}_{2 \mu \nu}^{a b}\left(x_{*}, y_{*} ; p_{\perp}\right)= & -\frac{g}{\alpha} \delta_{\mu}^{i} \delta_{\nu}^{j} \int_{y_{*}}^{x_{*}} d \frac{2}{s} \omega_{*}\left(\left[x_{*}, \omega_{*}\right] F_{i j}\left[\omega_{*}, y_{*}\right]\right)^{a b} \\
\mathfrak{G}_{3 \mu \nu}^{a b}\left(x_{*}, y_{*} ; p_{\perp}\right)= & \frac{g}{\alpha^{2} s}\left(\delta_{\mu}^{j} p_{2 \nu}+\delta_{\nu}^{j} p_{2 \mu}\right) \int_{y_{*}}^{x_{*}} d \frac{2}{s} \omega_{*}\left(\left[x_{*}, \omega_{*}\right] i D^{i} F_{i j}\left[\omega_{*}, y_{*}\right]\right)^{a b} \\
\mathfrak{G}_{4 \mu \nu}^{a b}\left(x_{*}, y_{*} ; p_{\perp}\right)= & -\frac{2 g^{2}}{\alpha^{2} s} \int_{y_{*}}^{x_{*}} d \frac{2}{s} \omega_{*} \int_{\omega_{*}}^{x_{*}} d \frac{2}{s} \omega_{*}^{\prime}\left(\delta_{\nu}^{j} p_{2 \mu}\left[x_{*}, \omega_{*}^{\prime}\right] F_{\bullet}^{i}\left[\omega_{*}^{\prime}, \omega_{*}\right] F_{i j}\left[\omega_{*}, y_{*}\right]\right. \\
& \left.+\delta_{\mu}^{j} p_{2 \nu}\left[x_{*}, \omega_{*}^{\prime}\right] F_{i j}\left[\omega_{*}^{\prime}, \omega_{*}\right] F_{\bullet}^{i}\left[\omega_{*}, y_{*}\right]\right)^{a b} .
\end{aligned}
$$

\section{B.3 Gluon propagator in the background of quark field}

The sub-eikonal corrections to the gluon propagator in the background of quark fields has been calculated in ref. [46]

$$
\begin{aligned}
\langle & \left.A_{\mu}^{a}(x) A_{\nu}^{b}(y)\right\rangle_{\psi, \bar{\psi}} \\
= & {\left[-\int_{0}^{+\infty} \frac{d \alpha}{2 \alpha} \theta\left(x_{*}-y_{*}\right)+\int_{-\infty}^{0} \frac{d \alpha}{2 \alpha} \theta\left(y_{*}-x_{*}\right)\right] e^{-i \alpha\left(x_{\bullet}-y_{\bullet}\right)} } \\
& \times g^{2} \int_{y_{*}}^{x_{*}} d \frac{2}{s} z_{1 *} \int_{y_{*}}^{z_{1 *}} d \frac{2}{s} z_{2 *} \frac{1}{4 \alpha} \int d^{2} z\left[\left\langle x_{\perp}\left|e^{-i \frac{\hat{p}_{\perp}^{2}}{\alpha s} x_{*}}\left(g_{\perp \mu}^{\xi}-\frac{s}{2} \frac{p_{2 \mu}}{\alpha} p_{\perp}^{\xi}\right)\right| z_{\perp}\right\rangle \bar{\psi}\left(z_{1 *}, z_{\perp}\right)\right. \\
& \times \gamma_{\xi}^{\perp} \not p_{1}\left[z_{1 *}, x_{*}\right]_{z} t^{a}\left[x_{*}, y_{*}\right]_{z} t^{b}\left[y_{*}, z_{2 *}\right]_{z} \gamma_{\perp}^{\sigma} \psi\left(z_{2 *}, z_{\perp}\right)\left\langle z_{\perp}\left|\left(g_{\sigma \nu}^{\perp}-p_{\sigma}^{\perp} \frac{s}{2} \frac{p_{2 \nu}}{\alpha}\right) e^{i \frac{\hat{p}_{\perp}^{2}}{\alpha s} y_{*}}\right| y_{\perp}\right\rangle \\
& +\left\langle y_{\perp}\left|e^{-i \frac{\hat{p}_{\perp}^{2}}{\alpha s} y_{*}}\left(g_{\perp \nu}^{\xi}-\frac{s}{2} \frac{p_{2 \nu}}{\alpha} p_{\perp}^{\xi}\right)\right| z_{\perp}\right\rangle \bar{\psi}\left(z_{2 *}, z_{\perp}\right) \gamma_{\xi}^{\perp} \not p_{1}\left[z_{2 *}, y_{*}\right]_{z} t^{b}\left[y_{*}, x_{*}\right]_{z} t^{a}\left[x_{*}, z_{1 *}\right]_{z} \gamma_{\perp}^{\sigma} \\
& \left.\times \psi\left(z_{1 *}, z_{\perp}\right)\left\langle z_{\perp}\left|\left(g_{\sigma \mu}^{\perp}-p_{\sigma}^{\perp} \frac{s}{2} \frac{p_{2 \mu}}{\alpha}\right) e^{i \frac{\hat{p}_{\perp}^{2}}{\alpha s} x_{*}}\right| x_{\perp}\right\rangle\right]+O\left(\lambda^{-2}\right)
\end{aligned}
$$

Note that the entire sub-eikonal correction is at the transverse position $z_{\perp}$. Moreover, in the shock-wave limit we are employing here, we have to send $x_{*} \rightarrow+\infty$ and $y_{*} \rightarrow-\infty$. In this limit the gauge link $\left[x_{*}, y_{*}\right]_{z}$ becomes the usual infinite Wilson line $U\left(z_{\perp}\right)$.

\section{Evaluation of matrix elements}

To understand which of the sub-eikonal term in the quark propagator (3.1) will contribute to the polarized structure functions we have to consider forward matrix elements with the sub-eikonal operators. In other words, we have to analyze the matrix element that will be obtained using the quark propagator (3.1) to calculate diagram in figure 3 for the impact factor. 
The polarization vector is a pseudo-vector which satisfy $S^{\mu} S_{\mu}=-1$ and $S \cdot P=0$. Let $S_{L}^{\mu}$ be the longitudinal component of the spin vector. In the DIS kinematics we have that $S_{L}^{\mu} \simeq \frac{\lambda}{M} P^{\mu}$ so, we may write $S^{\mu} \simeq \frac{\lambda}{M} P^{\mu}+S_{\perp}^{\mu}$.

We chose the proton momentum to be mainly in $p_{2}^{\mu}$ direction: $P^{\mu}=p_{2}^{\mu}+\frac{M^{2}}{s} p_{1}^{\mu}$, and the virtual photon has momentum $q^{\mu}=p_{1}^{\mu}-x_{B} p_{2}^{\mu}$.

Helicity is defined as $h=\lambda \frac{\vec{S} \cdot \vec{P}}{|\vec{P}|} \simeq \lambda g^{3 \mu} S_{\mu} \simeq-\lambda \frac{\sqrt{s}}{2 M}$ with $\lambda= \pm \frac{1}{2}$.

The components of the hadronic tensor $W^{\mu \nu}$, eq. (2.2), which are associated the polarized structure functions $g_{1}$ and $g_{2}$, are

$$
\begin{aligned}
W_{\mu \nu}^{\perp} & =-g_{\mu \nu}^{\perp} F_{1}+i \epsilon_{\mu \nu}^{\perp} g_{1} \\
g_{\mu}^{i} W_{\bullet i} & =i M x_{B} \epsilon_{\mu \nu}^{\perp} S_{\perp}^{\nu} g_{T}=-g_{\nu}^{i} W_{i \bullet} \\
g_{\mu}^{i} W_{* i} & =i \epsilon_{\mu \nu}^{\perp} S_{\perp}^{\nu} M g_{T}=-g_{\nu}^{i} W_{i *}
\end{aligned}
$$

where $g_{T}=g_{1}+g_{2}$ is the transverse polarized structure function. We see that the transverse hadronic tensor is associated to the $g_{1}$ structure function with longitudinal polarization, while transverse polarized structure function $g_{T}$ is obviously associated to the transverse $\operatorname{spin} S_{\perp}^{\mu}$.

To evaluate the matrix elements we will use spin vector $S^{\mu}$, the target momentum $P^{\mu}$, the direction of the Wilson line $\frac{2}{s} p_{1}^{\mu}$ and the transverse momentum $k_{\perp}^{\mu}$ conjugated to the size of the dipole $\Delta_{\perp}^{\mu}=(x-y)_{\perp}^{\mu}$. Note also that all distribution functions that we are going to introduce have dimensions $\left[M^{-2}\right]$.

\section{C.1 Matrix element with $\hat{\mathcal{O}}_{1}$ operator}

Here we are going to evaluate the matrix element that would be generated using the operator $\hat{\mathcal{O}}_{1}$ defined in eq. (B.13). All matrix elements will be of dipole type with the insertion of the sub-eikonal correction (see figure 3 ).

Let us consider the following matrix elements

$$
\begin{aligned}
& \left.\int d^{2} \Delta e^{i(\Delta, k)_{\perp}} \int_{-\infty}^{+\infty} d z_{*}\left\langle\langle P, S|\left[\operatorname{Tr}\left\{\left[\infty p_{1}, z_{*}\right]_{x} i g F^{i j}\left(z_{*}, x_{\perp}\right)\left[z_{*},-\infty p_{1}\right]_{x} U_{y}^{\dagger}\right\}+\text { a.c }\right] \mid P, S\right\rangle\right\rangle \\
& =a \frac{4}{s^{2}} \epsilon^{\bullet * \alpha i}\left(S_{*} P_{\bullet}-S_{\bullet} P_{*}\right) \frac{k_{\alpha}^{\perp} k^{j}}{M^{5}}+b \frac{4}{s^{2}} \epsilon^{\bullet * \alpha i} S_{\bullet} \frac{k_{\alpha}^{\perp} k^{j}}{M^{3}}+c \frac{4}{s^{2}} \epsilon^{\bullet * \alpha i} S_{\alpha}^{\perp} P_{\bullet} \frac{k^{j}}{M^{3}}+d \frac{k^{i} k^{j}}{M^{4}} \\
& =\frac{2}{s M^{2}} \epsilon^{k i} k^{j}\left(\frac{a}{M^{3}}\left(S_{*} P_{\bullet}-S_{\bullet} P_{*}\right) k_{k}+\frac{b}{M} S_{\bullet} k_{k}+\frac{c}{M} S_{k} P_{\bullet}\right)+d \frac{k^{i} k^{j}}{M^{4}} .
\end{aligned}
$$

with $a, b, c, d$ dimensionless coefficients. We now use $P^{\mu} \simeq\left(P^{0}, 0,0, P^{3}\right)=\frac{\sqrt{s}}{2}(1,0,0,-1)$, and $S \cdot P=0$, and

$$
\begin{aligned}
S_{*} P_{\bullet}-S_{\bullet} P_{*} & =\frac{s}{2}\left(S^{3} P^{0}-S^{0} P^{3}\right)=\frac{s}{2} \frac{M^{2} S^{3}}{P^{0}} \simeq \frac{s}{2} \frac{\lambda M P^{3}}{P^{0}}=-\frac{s}{2} \lambda M, \\
S_{\bullet} & \simeq \frac{1}{M} \lambda P \bullet=\lambda \frac{s}{2 M},
\end{aligned}
$$


where we also used $M S^{3} \simeq \lambda P^{3}$. The matrix element (C.4) becomes

$$
\begin{aligned}
& \left.\int d^{2} \Delta e^{i(\Delta, k)_{\perp}} \int_{-\infty}^{+\infty} d z_{*}\left\langle\langle P, S|\left[\operatorname{Tr}\left\{\left[\infty p_{1}, z_{*}\right]_{x} i g F^{i j}\left(z_{*}, x_{\perp}\right)\left[z_{*},-\infty p_{1}\right]_{x} U_{y}^{\dagger}\right\}+\text { a.c. }\right] \mid P, S\right\rangle\right\rangle \\
& =\frac{\epsilon^{k i} k^{j}}{M^{2}}\left(\lambda G_{L}\left(k_{\perp}^{2}, x\right) k_{k}+G_{T}\left(k_{\perp}^{2}, x\right) M S_{k}\right) .
\end{aligned}
$$

where we have introduced the polarized longitudinal (helicity) distribution function $G_{L}\left(k_{\perp}^{2}, x\right)$, the transverse gluon distribution function $G_{T}\left(k_{\perp}^{2}, x\right)$. As usual, Latin indexes assume values 1, 2, while Greek ones run from 0 to $3,(x, y)_{\perp}=x^{1} y^{1}+x^{2} y^{2}$.

Let us consider the matrix element generated by the term $\epsilon^{i j} F_{i j}$ in operator (B.13)

$$
\begin{aligned}
& \left.\int d^{2} \Delta e^{i(\Delta, k)_{\perp}} \int_{-\infty}^{+\infty} d z_{*}\left\langle\langle P, S|\left[\operatorname{Tr}\left\{\left[\infty p_{1}, z_{*}\right]_{x} i g \frac{s}{2} \epsilon^{i j} F_{i j}\left(z_{*}, x_{\perp}\right)\left[z_{*},-\infty p_{1}\right]_{x} U_{y}^{\dagger}\right\}+\text { a.c }\right] \mid P, S\right\rangle\right\rangle \\
& =\frac{s}{2}\left[\lambda \frac{k_{\perp}^{2}}{M^{2}} G_{L}\left(k_{\perp}^{2}, x\right)+\frac{(S, k)_{\perp}}{M} G_{T}\left(k_{\perp}^{2}, x\right)\right]
\end{aligned}
$$

So, the matrix element (C.8) will contribute to longitudinal polarized structure function, $g_{1}$, and to the transverse polarized structure function $g_{T}$.

Consider

$$
\begin{aligned}
& \int d^{2} \Delta e^{i(\Delta, k)_{\perp}} \int_{-\infty}^{+\infty} d \omega_{*} \int_{\omega_{*}}^{+\infty} d \omega_{*}^{\prime}\left(\omega_{*}-\omega_{*}^{\prime}\right) \\
& \left.\times\left\langle\langle P, S|\left[\operatorname{Tr}\left\{\left[\infty p_{1}, \omega_{*}^{\prime}\right]_{x} F^{i} \cdot\left[\omega_{*}^{\prime}, \omega_{*}\right]_{x} F_{i \bullet}\left[\omega_{*},-\infty p_{1}\right]_{x} U_{y}^{\dagger}\right\}+\text { a.c }\right] \mid P, S\right\rangle\right\rangle \\
& \quad=\frac{s}{2} \hat{G}\left(k_{\perp}^{2}, x\right)
\end{aligned}
$$

This matrix element will contribute to unpolarized structure function and $\hat{G}\left(k_{\perp}^{2}, x\right)$ is an unpolarized gluon distribution function.

Now we consider

$$
\begin{array}{ll}
\left.\int d^{2} \Delta e^{i(\Delta, k)_{\perp}} \int_{-\infty}^{+\infty} d \omega_{*} \omega_{*} k_{i}\left\langle\langle P, S|\left[\operatorname{Tr}\left\{\left[\infty p_{1}, \omega_{*}^{\prime}\right]_{x} F^{i} \bullet\left(\omega_{*}, x_{\perp}\right)\left[\omega_{*},-\infty p_{1}\right]_{x} U_{y}^{\dagger}\right\}+\text { a.c. }\right] \mid P, S\right\rangle\right\rangle \\
=\frac{s}{2} G^{-}\left(k_{\perp}^{2}, x\right)+\frac{s}{2} \frac{\hat{S} \times \vec{k}}{M} G_{T}^{-}\left(k_{\perp}^{2}, x\right) & \text { (C. } 10)
\end{array}
$$

Also this matrix element will contribute to unpolarized structure function with and $G^{-}\left(k_{\perp}^{2}, x\right)$ is an unpolarized gluon distribution function (recall that $F^{i} \bullet=\sqrt{\frac{s}{2}} F^{i-}$ ).

\section{C.2 Matrix element with $\hat{\mathcal{O}}_{j}$ operator}

Here we are going to evaluate the matrix element that would be generated using the operator $\hat{\mathcal{O}}_{j}$ defined in eq. (B.15).

Let us consider the term $\frac{1}{2} \not p_{2}\left[\hat{p}^{j}, \hat{\mathcal{O}}_{j}\right]$. One of the matrix element that it will generate is

$$
\begin{aligned}
& \int d^{2} \Delta e^{i(\Delta, k)_{\perp}} \int_{-\infty}^{+\infty} d \omega_{*} \int_{\omega_{*}}^{+\infty} d \omega_{*}^{\prime} \\
& \left.\times k^{j}\left\langle\langle P, S|\left[\operatorname{Tr}\left\{\left[\infty p_{1}, \omega_{*}^{\prime}\right]_{x} F^{k} \bullet\left[\omega_{*}^{\prime}, \omega_{*}\right]_{x} F_{k j}\left[\omega_{*},-\infty p_{1}\right]_{x} U_{y}^{\dagger}\right\}+\text { a.c. }\right] \mid P, S\right\rangle\right\rangle \\
& \quad=\frac{s}{2} k_{\perp}^{2} \bar{G}\left(k_{\perp}^{2}, x\right)+\frac{s}{2} M(\vec{S} \times \vec{k}) \bar{G}_{T}\left(k_{\perp}^{2}, x\right) .
\end{aligned}
$$


This matrix element will contribute to the unpolarized structure function with gluon distribution $\bar{G}\left(k_{\perp}^{2}, x\right)$ and to the transverse polarized structure function with transversely polarized gluon distribution $\bar{G}_{T}\left(k_{\perp}^{2}, x\right)$.

We also have matrix element

$$
\begin{aligned}
& \int d^{2} \Delta e^{i(\Delta, k)_{\perp}} \int_{-\infty}^{+\infty} d \omega_{*} \int_{\omega_{*}}^{+\infty} d \omega_{*}^{\prime} \\
& \left.\times\left\langle\langle P, S|\left[\operatorname{Tr}\left\{\left[\infty p_{1}, \omega_{*}^{\prime}\right]_{x} F_{\bullet *}\left[\omega_{*}^{\prime}, \omega_{*}\right]_{x} F_{j \bullet}\left[\omega_{*},-\infty p_{1}\right]_{x} U_{y}^{\dagger}\right\}+\text { a.c. }\right] \mid P, S\right\rangle\right\rangle \\
& \quad=\frac{s}{2} k_{j} \breve{G}\left(k_{\perp}^{2}, x\right)+\lambda \frac{s}{2} \epsilon^{i j} M S_{i} \breve{G}_{L}\left(k_{\perp}^{2}, x\right) .
\end{aligned}
$$

This matrix element will contribute to unpolarized structure function with gluon distribution $\breve{G}\left(k_{\perp}^{2}, x\right)$ and to the transverse polarized structure function with transversely polarized gluon distribution $\breve{G}_{T}\left(k_{\perp}^{2}, x\right)$.

\section{C.3 Matrix element with $\hat{\mathcal{O}}_{\bullet *}$ operator}

Let us now consider the forward matrix element obtained with operator $\hat{\mathcal{O}}_{\bullet}$ *

$$
\begin{gathered}
\left.\int d^{2} \Delta e^{i(\Delta, k)}\left\langle\langle P, S|\left[\operatorname{Tr}\left\{\left[\infty p_{1}, \omega_{*}\right]_{x} F_{\bullet *}\left(\omega_{*}, x_{\perp}\right)\left[\omega_{*},-\infty p_{1}\right]_{x} U_{y}^{\dagger}\right\}+\text { a.c. }\right] \mid P, S\right\rangle\right\rangle \\
=\frac{s}{2} \tilde{G}\left(k_{\perp}^{2}, x\right)+\frac{s}{2} \frac{\vec{S} \times \vec{k}}{M} \tilde{G}_{T}\left(k_{\perp}^{2}, x\right),
\end{gathered}
$$

where we recall that $F_{\bullet *}=\frac{s}{2} F^{-+}=\frac{s}{2} F_{+-}$. From this matrix element we have extracted an unpolarized gluon distribution $\tilde{G}\left(k_{\perp}^{2}, x\right)$, and a transversally polarized gluon distribution function $\tilde{G}_{T}\left(k_{\perp}^{2}, x\right)$. This matrix element will not contribute to $g_{1}$, but to $g_{T}$.

\section{C.4 Quark propagator for $g_{1}$ structure function}

From the analysis just performed we may conclude that the gluon field sub-eikonal contribution to the quark propagator that we have to use to calculate the impact factor for polarized DIS and relevant for $g_{1}$ structure function is

$$
\begin{aligned}
\left\langle x\left|\frac{i}{\hat{p}+i \epsilon}\right| y\right\rangle \ni & {\left[\int_{0}^{+\infty} \frac{d \alpha}{2 \alpha} \theta\left(x_{*}-y_{*}\right)-\int_{-\infty}^{0} \frac{d \alpha}{2 \alpha} \theta\left(y_{*}-x_{*}\right)\right] e^{-i \alpha\left(x_{\bullet}-y_{\bullet}\right)} \frac{1}{\alpha s} } \\
& \times \int d^{2} z\left\langle x_{\perp}\left|e^{-i \frac{\hat{p}_{\perp}^{2}}{\alpha s} x_{*}} \not p\right| z_{\perp}\right\rangle \\
& \times\left\{\not p_{2}\left[x_{*}, y_{*}\right]_{z}+\frac{i g}{2 \alpha} \int_{y_{*}}^{x_{*}} d \frac{2}{s} \omega_{*}\left[x_{*}, \omega_{*}\right]_{z} \frac{1}{2} \not p_{2} \gamma^{5} \epsilon^{i j} F_{i j}\left[\omega_{*}, y_{*}\right]_{z}\right\} \\
& \times\left\langle z_{\perp}\left|\not p e^{i \frac{\hat{p}_{\perp}^{2}}{\alpha s} y_{*}}\right| y_{\perp}\right\rangle
\end{aligned}
$$

where the symbol $\ni$ in eq. (C.14) means that the terms in the right-hand-side (r.h.s.) are only part of all the terms of the quark propagators; the terms we have left out will not contribute to the calculation of the impact factor for polarized $g_{1}$ structure function. It should be stressed that here we are only concerned with the sub-eikonal correction with only gluon background field. Propagator (C.14) will be used to calculate the impact factor diagram in figure $1 \mathrm{~b}$. To calculate the impact factor diagram in figure $1 \mathrm{~b}$ we need the quark propagator with quarks in the background given in eq. (3.7). 


\section{Derivation of the OPE with quark-sub-eikonal corrections}

Here we provide some calculation details of the derivation of the OPE with quark-subeikonal corrections eq. (4.2). Let us consider the $T$-product of two electromagnetic currents am perform the functional integration over the spinor fields. As usual, we start with the case in which $x^{+}>0>y^{+}$. Since the sub-eikonal correction can be included either in the quark fermion line or in the anti-quark fermion line, at sub-eikonal level we have, without exceeding our precision, two terms

$$
\left\langle\mathrm{T}\left\{\hat{j}^{\mu}(x) \hat{j}^{\nu}(y)\right\}\right\rangle_{A, \psi, \bar{\psi}} \stackrel{x^{+}>0>y^{+}}{=}-\operatorname{tr}\left\{\gamma^{\mu}\langle\psi(x) \bar{\psi}(y)\rangle_{A, \psi, \bar{\psi}} \gamma^{\nu}\langle\psi(y) \bar{\psi}(x)\rangle_{A, \psi, \bar{\psi}}\right\}
$$

Now we are concerned only with the contribution coming from quarks in the external field, so using the quark propagator (3.8) we have

$$
\begin{aligned}
& \left\langle\mathrm{T}\left\{\hat{j}^{\mu}(x) \hat{j}^{\nu}(y)\right\}\right\rangle_{A, \psi, \bar{\psi}} \\
& \stackrel{x^{+}>0>y^{+}}{\ni}-\operatorname{Tr} \operatorname{tr}\left\{\gamma^{\mu} \frac{-g^{2}}{16 \pi^{3} x_{*}^{2} y_{*}^{2}} \int_{y_{*}}^{x_{*}} d z_{*} \int_{y_{*}}^{z_{*}} d z_{*}^{\prime} \int d^{2} z_{2}\right. \\
& \times \frac{X_{2}\left[x_{*}, z_{*}\right]_{z_{2}} t^{a} \gamma_{\perp}^{\alpha} \psi\left(z_{*}, z_{2 \perp}\right)\left[z_{*}, z_{*}^{\prime}\right]_{z_{2}}^{a b} \bar{\psi}\left(z_{*}^{\prime}, z_{2 \perp}\right) \gamma_{\alpha}^{\perp} t^{b}\left[z_{*}^{\prime}, y_{*}\right]_{z_{2}} Y_{2}}{\left(\mathcal{Z}_{2}+i \epsilon\right)^{2}} \\
& \left.\times \gamma^{\nu} \frac{i}{2 \pi^{3} x_{*}^{2} y_{*}^{2}} \int d^{2} z_{1} \frac{Y_{1} \not p_{2}\left[y_{*}, x_{*}\right]_{z_{1}} X_{1}}{\left(\mathcal{Z}_{1}+i \epsilon\right)^{3}}\right\}-\operatorname{Tr} \operatorname{tr}\left\{\gamma^{\mu} \frac{-i}{2 \pi^{3} x_{*}^{2} y_{*}^{2}}\right. \\
& \times \int d^{2} z_{1} \frac{X_{1} \not p_{2}\left[x_{*}, y_{*}\right]_{z_{1}} Y_{1}}{\left(\mathcal{Z}_{1}+i \epsilon\right)^{3}} \gamma^{\nu} \frac{-g^{2}}{16 \pi^{3} x_{*}^{2} y_{*}^{2}} \int_{x_{*}}^{y_{*}} d z_{*}^{\prime} \int_{x_{*}}^{z_{*}^{\prime}} d z_{*} \int d^{2} z_{2} \frac{1}{\left(\mathcal{Z}_{2}+i \epsilon\right)^{2}} \\
& \left.\times Y_{2}\left[y_{*}, z_{*}^{\prime}\right]_{z_{2}} t^{a} \gamma_{\perp}^{\alpha} \psi\left(z_{*}^{\prime}, z_{2 \perp}\right)\left[z_{*}^{\prime}, z_{*}\right]_{z_{2}}^{a b} \bar{\psi}\left(z_{*}, z_{2 \perp}\right) \gamma_{\alpha}^{\perp} t^{b}\left[z_{*}, x_{*}\right]_{z_{2}} X_{2}\right\}
\end{aligned}
$$

The two terms in (D.2) represent the sub-eikonal correction for quark and anti-quark propagator. We can further simplify (D.2) by renaming dummy variables

$$
\begin{aligned}
& \left\langle\mathrm{T}\left\{\hat{j}^{\mu}(x) \hat{j}^{\nu}(y)\right\}\right\rangle_{A, \psi, \bar{\psi}} \\
& \underset{x^{+}>0>y^{+}}{\ni} \frac{i g^{2}}{32 \pi^{6} x_{*}^{4} y_{*}^{4}} \int d^{2} z_{2} d^{2} z_{1}\left(\mathcal{Z}_{2}+i \epsilon\right)^{-2}\left(\mathcal{Z}_{1}+i \epsilon\right)^{-3} \\
& \quad \times\left[\int _ { y _ { * } } ^ { x _ { * } } d z _ { * } \int _ { y _ { * } } ^ { z _ { * } } d z _ { * } ^ { \prime } \operatorname { T r } \operatorname { t r } \left\{\gamma^{\mu} X_{2}\left[x_{*}, z_{*}\right]_{z_{2}} t^{a} \gamma_{\perp}^{\alpha} \psi\left(z_{*}, z_{2 \perp}\right)\left[z_{*}, z_{*}^{\prime}\right]_{z_{2}}^{a b} \bar{\psi}\left(z_{*}^{\prime}, z_{2 \perp}\right)\right.\right. \\
& \left.\quad \times \gamma_{\alpha}^{\perp} t^{b}\left[z_{*}^{\prime}, y_{*}\right]_{z_{2}} Y_{2} \gamma^{\nu} Y_{1} \not \not_{2}\left[y_{*}, x_{*}\right]_{z_{1}} X_{1}\right\} \\
& \quad-\int_{x_{*}}^{y_{*}} d z_{*} \int_{x_{*}}^{z_{*}} d z_{*}^{\prime} \operatorname{Tr} \operatorname{tr}\left\{\gamma^{\mu} X_{1} \not \phi_{2}\left[x_{*}, y_{*}\right]_{z_{1}} Y_{1} \gamma^{\nu} Y_{2}\left[y_{*}, z_{*}\right]_{z_{2}} t^{a} \gamma_{\perp}^{\alpha} \psi\left(z_{*}, z_{2 \perp}\right) \bar{\psi}\left(z_{*}^{\prime}, z_{2 \perp}\right)\right. \\
& \left.\left.\quad \times\left[z_{*}, z_{*}^{\prime}\right]_{z_{2}}^{a b} \gamma_{\alpha}^{\perp} t^{b}\left[z_{*}^{\prime}, x_{*}\right]_{z_{2}} X_{2}\right\}\right]
\end{aligned}
$$


Observing that last line in (D.3) can be written as the adjoint conjugation of the second, we have

$$
\begin{aligned}
& \left\langle\mathrm{T}\left\{\hat{j}^{\mu}(x) \hat{j}^{\nu}(y)\right\}\right\rangle_{A, \psi, \bar{\psi}} \\
& \stackrel{x^{+}>0>y^{+}}{\ni} \frac{i g^{2}}{32 \pi^{6} x_{*}^{4} y_{*}^{4}} \int d^{2} \omega d^{2} z\left(\mathcal{Z}_{2}+i \epsilon\right)^{-2}\left(\mathcal{Z}_{1}+i \epsilon\right)^{-3} \\
& \times\left[\int _ { y _ { * } } ^ { x _ { * } } d z _ { * } \int _ { y _ { * } } ^ { z _ { * } } d z _ { * } ^ { \prime } \operatorname { T r } \operatorname { t r } \left\{\gamma^{\mu} X_{2}\left[x_{*}, z_{*}\right]_{z_{2}} t^{a} \gamma_{\perp}^{\alpha} \psi\left(z_{*}, z_{2 \perp}\right)\left[z_{*}, z_{*}^{\prime}\right]_{z_{2}}^{a b} \bar{\psi}\left(z_{*}^{\prime}, z_{2 \perp}\right)\right.\right. \\
& \left.\times \gamma_{\alpha}^{\perp} t^{b}\left[z_{*}^{\prime}, y_{*}\right]_{z_{2}} Y_{2} \gamma^{\nu} Y_{1} \not p_{2}\left[y_{*}, x_{*}\right]_{z_{1}} X_{1}\right\} \\
& -\left(\int _ { y _ { * } } ^ { x _ { * } } d z _ { * } \int _ { y _ { * } } ^ { z _ { * } } d z _ { * } ^ { \prime } \operatorname { T r } \operatorname { t r } \left\{\gamma^{\mu} X_{2}\left[x_{*}, z_{*}\right]_{z_{2}} t^{a} \gamma_{\perp}^{\alpha} \psi\left(z_{*}, z_{2 \perp}\right)\left[z_{*}, z_{*}^{\prime}\right]_{z_{2}}^{a b} \bar{\psi}\left(z_{*}^{\prime}, z_{2 \perp}\right)\right.\right. \\
& \left.\left.\left.\times \gamma_{\alpha}^{\perp} t^{b}\left[z_{*}^{\prime}, y_{*}\right]_{z_{2}} Y_{2} \gamma^{\nu} Y_{1} \not p_{2}\left[y_{*}, x_{*}\right]_{z_{1}} X_{1}\right\}\right)^{\dagger}\right] \text {. }
\end{aligned}
$$

Now, using definition of operator $Q_{i j}^{\alpha \beta}$ in eq. (3.9) we finally get eq. (4.2)

$$
\begin{aligned}
&\left\langle\mathrm{T}\left\{j^{\mu}(x) j^{\nu}(y)\right\}\right\rangle_{A, \psi, \bar{\psi}} \stackrel{x_{*}>0>y_{*}}{\ni} \frac{i}{32 \pi^{6} x_{*}^{4} y_{*}^{4}} \int \frac{d^{2} z_{1} d^{2} z_{2}}{\left[\mathcal{Z}_{1}+i \epsilon\right]^{3}\left[\mathcal{Z}_{2}+i \epsilon\right]^{2}} \\
& \times\left[\operatorname{Tr} \operatorname{tr}\left\{\gamma^{\mu} X_{2} \gamma_{\perp}^{\rho} Q\left(z_{2 \perp}\right) \gamma_{\rho}^{\perp} Y_{2} \gamma^{\nu} Y_{1} \not p_{2} U_{z_{1}}^{\dagger} X_{1}\right\}\right. \\
&\left.-\left(\operatorname{Tr} \operatorname{tr}\left\{\gamma^{\mu} X_{2} \gamma_{\perp}^{\rho} Q\left(z_{2 \perp}\right) \gamma_{\rho}^{\perp} Y_{2} \gamma^{\nu} Y_{1} \not p_{2} U_{z_{1}}^{\dagger} X_{1}\right\}\right)^{\dagger}\right]
\end{aligned}
$$

\section{E Coefficients $I_{1}^{\mu \nu}$ and $I_{5}^{\mu \nu}$}

Here we provide explicit expressions for the coefficients $I_{1}^{\mu \nu}$ and $I_{5}^{\mu \nu}$. We remind that $X_{i}^{\mu}=X_{i \perp}^{\mu}+\frac{2}{s} x_{*} p_{1}^{\mu}$ and the same for $y$ with $i=1,2, X_{1 \perp}^{\mu}=\left(x-z_{1}\right)_{\perp}^{\mu}$ and $X_{2 \perp}^{\mu}=\left(x-z_{2}\right)_{\perp}^{\mu}$ and similar expression with $y$.

Coefficient $I_{1}^{\mu \nu}$ is

$$
\begin{aligned}
I_{1}^{\mu \nu}=\frac{1}{2}\left[2 x_{*} y_{*}\left(z_{12 \perp}^{2} g^{\mu \nu}+z_{12}^{\mu} z_{12}^{\nu}-\left(X_{1}+X_{2}\right)^{\mu}\left(Y_{1}+Y_{2}\right)^{\nu}\right)\right. & -2 p_{2}^{\mu} p_{2}^{\nu}\left(\left(X_{1} \cdot Y_{1}\right)\left(X_{2} \cdot Y_{2}\right)-\left(X_{1} \cdot Y_{2}\right)\left(X_{2} \cdot Y_{1}\right)+\left(Y_{1} \cdot Y_{2}\right)\left(X_{1} \cdot X_{2}\right)\right) \\
& -x_{*} p_{2}^{\nu}\left(-\left(Y_{1}^{2}-Y_{2}^{2}\right) z_{12 \perp}^{\mu}+z_{12 \perp}^{2}\left(Y_{1}^{\mu}+Y_{2}^{\mu}\right)-2 Y_{1} \cdot Y_{2}\left(X_{1}^{\mu}+X_{2}^{\mu}\right)\right) \\
& \left.-y_{*} p_{2}^{\mu}\left(-\left(X_{1}^{2}-X_{2}^{2}\right) z_{12 \perp}^{\nu}+z_{12 \perp}^{2}\left(X_{1}^{\nu}+X_{2}^{\nu}\right)-2 X_{1} \cdot X_{2}\left(Y_{1}^{\nu}+Y_{2}^{\nu}\right)\right)\right] \\
=\frac{1}{2} & x_{*}^{2} y_{*}^{2} \frac{\partial^{2}}{\partial x_{\mu} \partial y_{\nu}}\left(\mathcal{Z}_{1} \mathcal{Z}_{2}-z_{12 \perp}^{2} \frac{(x-y)^{2}}{x_{*} y_{*}}\right)
\end{aligned}
$$




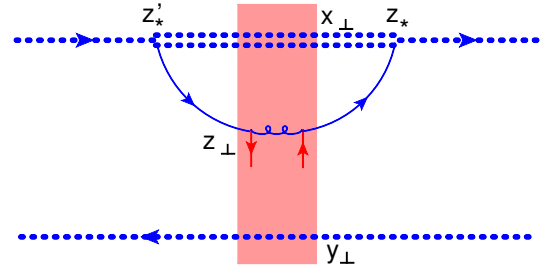

a)

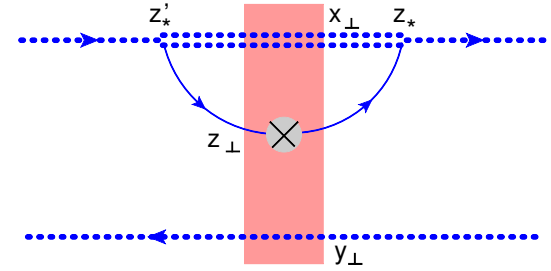

b)

Figure 14. Diagrams with quantum quarks. Blue fermion lines are quantum fields, while red fermion lines are classical fields. The gray circle with a cross on it represents here the $\epsilon^{i j} F_{i j}$ classical field.

Coefficient $I_{5}^{\mu \nu}$ is

$$
\begin{aligned}
I_{5}^{\mu \nu}=\left[x_{*} y_{*}\left(Y_{1 j} X_{1 k}-Y_{2 j} X_{2 k}\right)\left(g_{\perp}^{\mu \nu} \epsilon^{j k}+\epsilon^{\mu \nu} g^{j k}\right)\right. \\
\quad+p_{2}^{\mu} p_{2}^{\nu}\left(\left(Y_{1}, Y_{2}\right) \vec{X}_{1} \times \vec{X}_{2}-\left(X_{1}, X_{2}\right) \vec{Y}_{1} \times \vec{Y}_{2}\right) \\
+x_{*} y_{*}\left(\left(Y_{1 \perp}^{\nu} X_{2 j}-Y_{2 \perp}^{\nu} X_{1 j}\right) \epsilon^{j \mu}-\left(X_{1 \perp}^{\mu} Y_{2 j}-X_{2 \perp}^{\mu} Y_{1 j}\right) \epsilon^{j \nu}\right) \\
\quad-x_{*} p_{2}^{\nu}\left(\left(Y_{1}, Y_{2}\right)\left(X_{1 k}-X_{2 k}\right) \epsilon^{k \mu}+\left(\vec{Y}_{1} \times \vec{Y}_{2}\right)\left(X_{1 \perp}^{\mu}+X_{2 \perp}^{\mu}\right)\right) \\
+y_{*} p_{2}^{\mu}\left(\left(X_{1}, X_{2}\right)\left(Y_{1 k}-Y_{2 k}\right) \epsilon^{k \nu}+\left(\vec{X}_{1} \times \vec{X}_{2}\right)\left(Y_{1 \perp}^{\nu}+Y_{2 \perp}^{\nu}\right)\right) \\
\quad+\frac{4}{s}\left[x_{*} y_{*}^{2} p_{1}^{\nu}\left(X_{2 j}-X_{1 j}\right) \epsilon^{j \mu}-x_{*}^{2} y_{*} p_{1}^{\mu}\left(Y_{2 j}-Y_{1 j}\right) \epsilon^{j \nu}\right. \\
\left.\left.\quad+y_{*}^{2} p_{1}^{\nu} p_{2}^{\mu} \vec{X}_{1} \times \vec{X}_{2}-x_{*}^{2} p_{1}^{\mu} p_{2}^{\nu} \vec{Y}_{1} \times \vec{Y}_{2}\right]\right] \\
=\left(x_{*} \partial_{x}^{\mu}-p_{2}^{\mu}\right)\left(y_{*} \partial_{y}^{\nu}-p_{2}^{\nu}\right)\left[\left(\vec{Y}_{1} \times \vec{Y}_{2}\right) X_{1} \cdot X_{2}-\left(\vec{X}_{1} \times \vec{X}_{2}\right) Y_{1} \cdot Y_{2}\right]
\end{aligned}
$$

\section{F Evolution equation for operators $\mathcal{Q}_{1 x}$ and $\mathcal{Q}_{5 x}$}

\section{F.1 Diagrams with $\mathcal{Q}_{1 x}$ and $\mathcal{Q}_{5 x}$ quantum}

It is convenient to calculate the evolution of the operator $Q_{i j}^{\alpha \beta}$ given in eq. (3.9). Using the following relations

$$
\begin{aligned}
& \mathcal{Q}_{5}\left(x_{\perp}\right)=\operatorname{tr}\left\{\gamma^{5} \not p_{1} Q_{x}\right\} \\
& \mathcal{Q}_{1}\left(x_{\perp}\right)=\operatorname{tr}\left\{i \not p_{1} Q_{x}\right\}
\end{aligned}
$$

we will obtain the evolution equations for $\operatorname{Tr}\left\{\mathcal{Q}_{1 x} U_{y}^{\dagger}\right\}$ and $\operatorname{Tr}\left\{\mathcal{Q}_{5 x} U_{y}^{\dagger}\right\}$. We will use tr for traces over Dirac matrices, Tr for traces over color matrices in the fundamental representation, and $\operatorname{Tr}_{\mathrm{a}}$ for traces in the adjoint representation.

In this section, we will calculate the evolution of the operator $Q_{i j}^{\alpha \beta}$ as defined in eq. (3.9). Let us start calculating diagram in figure 14a. Performing the contraction 
over the quark field we have

$$
\left\langle\operatorname{Tr}\left\{Q_{x}^{\beta \alpha} U_{y}^{\dagger}\right\}\right\rangle_{\text {Figure } 14 a}=g^{2} \int_{0}^{+\infty} d z_{*} \int_{-\infty}^{0} d z_{*}^{\prime} t_{i j}^{a} U_{\omega}^{a b}\left(t^{b} U_{z}^{\dagger}\right)_{k i}\left\langle\psi_{j}^{\beta}\left(z_{*}, \omega_{\perp}\right) \bar{\psi}_{k}^{\alpha}\left(z_{*}^{\prime}, \omega_{\perp}\right)\right\rangle
$$

To proceed with the calculation for diagram in figure 14a we need the quark propagator with quark sub-eikonal correction (3.8), and obtain

$$
\begin{aligned}
&\langle\left.\operatorname{Tr}\left\{Q_{x}^{\beta \alpha} U_{y}^{\dagger}\right\}\right\rangle_{\text {Figure } 14 a} \\
&=-\alpha_{s} \int_{0}^{+\infty} \frac{d \alpha}{\alpha} \int d^{2} z d^{2} q_{1} d^{2} q_{2} e^{i\left(q_{1}, x-z\right)+i\left(q_{2}, z-x\right)} U_{x}^{a b} \frac{\left(\phi_{1 \perp} \gamma_{\rho}^{\perp}\right)_{\beta \beta^{\prime}}}{q_{1 \perp}^{2}} \\
& \times \operatorname{Tr}\left\{t^{a} Q_{z}^{\beta^{\prime} \alpha^{\prime}} t^{b} U_{y}^{\dagger}\right\} \frac{\left(\gamma_{\perp}^{\rho} q_{2 \perp}\right)_{\alpha^{\prime} \alpha}}{q_{2 \perp}^{2}} \\
&= \frac{\alpha_{s}}{4 \pi^{2}} \int_{0}^{+\infty} \frac{d \alpha}{\alpha} \int d^{2} z U_{x}^{a b} \frac{\left[(\not x-\not)^{\perp} \gamma_{\rho}^{\perp}\right]_{\beta \beta^{\prime}}}{(x-z)_{\perp}^{2}} \operatorname{Tr}\left\{t^{a} Q_{z}^{\beta^{\prime} \alpha^{\prime}} t^{b} U_{y}^{\dagger}\right\} \frac{\left[\gamma_{\perp}^{\rho}(\not z-\not x)_{\perp}\right]_{\alpha^{\prime} \alpha}}{(x-z)_{\perp}} \\
&= \frac{\alpha_{s}}{4 \pi^{2}} \int_{0}^{+\infty} \frac{d \alpha}{\alpha} \int d^{2} z \frac{\left[(\not x-\not)_{\perp} \gamma_{\rho}^{\perp}\right]_{\beta \beta^{\prime}}}{(x-z)_{\perp}^{2}}\left[\frac{1}{2} \operatorname{Tr}\left\{U_{y}^{\dagger} U_{x}\right\} \operatorname{Tr}\left\{U_{x}^{\dagger} Q_{z}^{\beta^{\prime} \alpha^{\prime}}\right\}\right. \\
&\left.\quad-\frac{1}{2 N_{c}} \operatorname{Tr}\left\{U_{y}^{\dagger} Q_{z}^{\beta^{\prime} \alpha^{\prime}}\right\}\right] \frac{\left[\gamma_{\perp}^{\rho}(\not z-\not \not)_{\perp}\right]_{\alpha^{\prime} \alpha}}{(x-z)_{\perp}}
\end{aligned}
$$

We perform same steps for diagram in figure $14 \mathrm{~b}$ but this time we need the gluon subeikonal contribution to the quark propagator. Thus, we have

$$
\begin{aligned}
\left\langle\operatorname{Tr}\left\{Q_{x}^{\beta \alpha} U_{y}^{\dagger}\right\}\right\rangle_{\text {Figure } 14 b}= & \frac{\alpha_{s}}{4 \pi^{2}} \int_{0}^{+\infty} \frac{d \alpha}{\alpha} \int d^{2} z \frac{\left[(\not x-\not \not)_{\perp} \gamma_{\perp}^{\rho}\right]_{\beta \beta^{\prime}}}{(z-x)_{\perp}^{2}} \frac{1}{2 s}\left[\not \phi_{2} \gamma^{5}\right]^{\beta^{\prime} \alpha^{\prime}} \\
& \times \operatorname{Tr}\left\{t^{a} \mathcal{F}_{z} t^{b} U_{y}^{\dagger}\right\} U_{x}^{a b} \frac{\left[\gamma_{\rho}^{\perp}(\not z-\not x)_{\perp}\right]_{\alpha^{\prime} \alpha}}{(z-x)_{\perp}}
\end{aligned}
$$

Contracting eqs. (F.4) and (F.5) with $\not p_{1} \gamma^{5}$ and summing them up we have

$$
\begin{aligned}
& \left\langle\operatorname{Tr}\left\{\mathcal{Q}_{5 x} U_{y}^{\dagger}\right\}\right\rangle_{\text {Figure } 14} \\
& =\left\langle\operatorname{Tr}\left\{\operatorname{tr}\left\{\gamma^{5} \not p_{1} Q_{x}\right\} U_{y}^{\dagger}\right\}\right\rangle_{\text {Figure } 14} \\
& =\frac{\alpha_{s}}{4 \pi^{2}} \int_{0}^{+\infty} \frac{d \alpha}{\alpha} \int d^{2} z \operatorname{tr}\left\{\gamma^{5} \not p_{1} \frac{\left[(\not-\not)_{\perp} \gamma_{\rho}^{\perp}\right]}{(x-z)_{\perp}^{2}} U_{x}^{a b}\right. \\
& \left.\quad \times \operatorname{Tr}\left\{t^{a}\left(Q_{z}+\frac{1}{2 s}\left[\not p_{2} \gamma^{5}\right] \mathcal{F}_{z}\right) t^{b} U_{y}^{\dagger}\right\} \frac{\left[\gamma_{\perp}^{\rho}(\not-\not x)_{\perp}\right]}{(x-z)_{\perp}^{2}}\right\} \\
& =\frac{\alpha_{s}}{2 \pi^{2}} \int_{0}^{+\infty} \frac{d \alpha}{\alpha} \int d^{2} z \frac{U_{x}^{a b}}{(x-z)_{\perp}^{2}} \operatorname{Tr}\left\{t^{a}\left(\mathcal{Q}_{5 z}+\mathcal{F}_{z}\right) t^{b} U_{y}^{\dagger}\right\} \\
& =\frac{\alpha_{s}}{4 \pi^{2}} \int_{0}^{+\infty} \frac{d \alpha}{\alpha} \int \frac{d^{2} z}{(x-z)_{\perp}^{2}}\left[\operatorname{Tr}\left\{\left(\mathcal{Q}_{5 z}+\mathcal{F}_{z}\right) U_{x}^{\dagger}\right\} \operatorname{Tr}\left\{U_{y}^{\dagger} U_{x}\right\}-\frac{1}{N_{c}} \operatorname{Tr}\left\{\left(\mathcal{Q}_{5 z}+\mathcal{F}_{z}\right) U_{y}^{\dagger}\right\}\right]
\end{aligned}
$$


While contracting eqs. (F.4) and (F.5) with $i \not p_{1}$ and again summing them up we obtain

$$
\begin{aligned}
& \left\langle\operatorname{Tr}\left\{\mathcal{Q}_{1 x} U_{y}^{\dagger}\right\}\right\rangle_{\text {Figure 14 }} \\
& =\left\langle\operatorname{Tr}\left\{\operatorname{tr}\left\{i \not p_{1} Q_{x}\right\} U_{y}^{\dagger}\right\}\right\rangle_{\text {Figure } 14} \\
& =\frac{\alpha_{s}}{4 \pi^{2}} \int_{0}^{+\infty} \frac{d \alpha}{\alpha} \int d^{2} z \operatorname{tr}\left\{i \not p_{1} \frac{\left[(\not p-\not x)_{\perp} \gamma_{\rho}^{\perp}\right]}{(x-z)_{\perp}^{2}}\right. \\
& \left.\quad \times U_{x}^{a b} \operatorname{Tr}\left\{t^{a}\left(Q_{z}+\frac{1}{2 s}\left[\not p_{2} \gamma^{5}\right] \mathcal{F}_{z}\right) t^{b} U_{y}^{\dagger}\right\} \frac{\left[\gamma_{\perp}^{\rho}(\not p-\not x)_{\perp}\right]}{(x-z)_{\perp}^{2}}\right\}
\end{aligned}
$$

Using $\operatorname{tr}\left\{(\not x-\not)_{\perp} \gamma_{\rho}^{\perp} \not p_{2} \sigma^{i j} \gamma_{\perp}^{\rho}(\not k-\not x)_{\perp} \not \phi_{1}\right\}=0$, we see that the term proportional to $\mathcal{F}_{z}$ cancels, so we do not have mixing of operator of different parity. Thus, we arrive at

$$
\begin{aligned}
\langle\operatorname{Tr} & \left.\left\{\mathcal{Q}_{1 x} U_{y}^{\dagger}\right\}\right\rangle_{\text {Figure } 14} \\
& =\frac{\alpha_{s}}{2 \pi^{2}} \int_{0}^{+\infty} \frac{d \alpha}{\alpha} \int d^{2} z \frac{U_{x}^{a b}}{(x-z)_{\perp}^{2}} \operatorname{Tr}\left\{t^{a} \mathcal{Q}_{1 z} t^{b} U_{y}^{\dagger}\right\} \\
& =\frac{\alpha_{s}}{4 \pi^{2}} \int_{0}^{+\infty} \frac{d \alpha}{\alpha} \int d^{2} z \frac{1}{(x-z)_{\perp}^{2}}\left[\operatorname{Tr}\left\{U_{x}^{\dagger} \mathcal{Q}_{1 z}\right\} \operatorname{Tr}\left\{U_{y}^{\dagger} U_{x}\right\}-\frac{1}{N_{c}} \operatorname{Tr}\left\{\mathcal{Q}_{1 z} U_{y}^{\dagger}\right\}\right]
\end{aligned}
$$

To arrive at results (F.6) and (F.8) we used identities

$$
\begin{aligned}
\not p_{2} \sigma^{i j} & =\epsilon^{i j} \not p_{2} \gamma^{5} \\
\operatorname{tr}\left\{(\not p-\not)_{\perp} \gamma_{\rho}^{\perp} \not p_{2} \sigma^{i j} \gamma_{\perp}^{\rho}(\not-\not x)_{\perp} \gamma^{5} \not p_{1}\right\} & =4 s \epsilon^{i j}(x-z)_{\perp}^{2}, \\
\operatorname{tr}\left\{\gamma^{5} \not p_{1}(\not x-\not)_{\perp} \gamma_{\rho}^{\perp} \psi \bar{\psi} \gamma_{\perp}^{\rho}(\not-\not)_{\perp}\right\} & =2(x-z)_{\perp}^{2} \operatorname{tr}\left\{\gamma^{5} \not p_{1} \psi \bar{\psi}\right\}, \\
\operatorname{tr}\left\{\not p_{1}(\not \not-\not)_{\perp} \gamma_{\rho}^{\perp} \psi \bar{\psi} \gamma_{\perp}^{\rho}(\not-\not x)_{\perp}\right\} & =2(x-z)_{\perp}^{2} \operatorname{tr}\left\{\not p_{1} \psi \bar{\psi}\right\},
\end{aligned}
$$

The evolution equations (F.6) and (F.8) are not closed evolution equations because after one loop evolution we have generated new operators. Consequently, to solve them we should find the evolution equations of the operators generated after one loop, thus generating a hierarchy of evolution equations similar to the Balitsky-hierarchy equations for dipoles. Alternatively, one can try to truncate the hierarchy of evolution equations employing some approximation. For example, it is known that in the large $N_{c}$ limit the Balitskyhierarchy of evolution equations is truncated to the BK equation. In order to perform a similar truncation in eqs. (F.6) and (F.8) it is probably convenient to work out the color algebra and reduce all the operators in the fundamental representation, as it is done in eq. (6.50), and only then one can try to find a way to linearize and solve the evolution equations.

It is interesting to notice that if we consider the evolution equation of the sum of operator $\hat{\mathcal{Q}}_{5 x}$, eq. (7.10), of operator $\hat{\mathcal{F}}_{x}$, eq. (6.49) and their adjoint conjugated we obtain and neglecting the quark-to-gluon diagrams and consider only the terms contributing to 


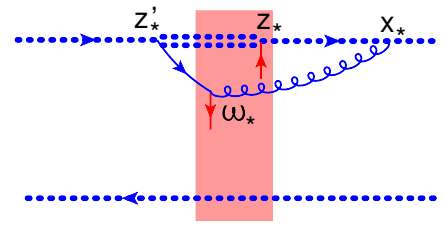

a)

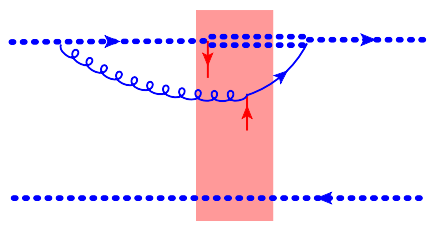

c)

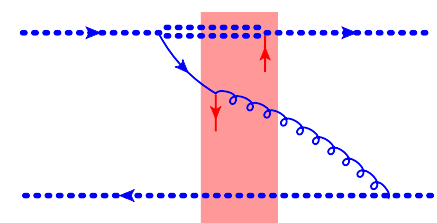

b)

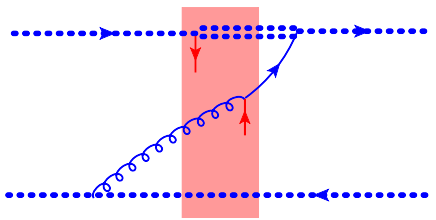

d)

Figure 15. One loop correction diagrams to the operator $Q_{i j}^{\alpha \beta}$ defined in eq. (3.9) using the quarkto-gluon propagator. Single dotted lines are Wilson-line in the fundamental representation, while double dotted lines are Wilson-line in the adjoint representation.

the double logarithm, we have

$$
\begin{aligned}
\left\langle\operatorname{Tr}\left\{\left(\mathcal{F}_{x}+\mathcal{Q}_{5 x}\right) U_{y}^{\dagger}\right\}+\operatorname{Tr}\left\{\left(\mathcal{F}_{x}^{\dagger}+\mathcal{Q}_{5 x}^{\dagger}\right) U_{y}\right\}\right\rangle_{\text {Figures }} 9+10+14 \\
=\frac{\alpha_{s}}{\pi^{2}} \int_{0}^{+\infty} \frac{d \alpha}{\alpha} \int \frac{d^{2} z}{(x-z)_{\perp}^{2}}\left\{\operatorname{Tr}\left\{U_{x} t^{a} U_{y}^{\dagger} t^{b}\right\}\left(\mathcal{Q}_{5 z}^{b a}+\mathcal{Q}_{5 z}^{b a \dagger}+\mathcal{F}_{z}^{b a}\right)\right. \\
\quad+\operatorname{Tr}\left\{t^{b} U_{y} t^{a} U_{x}^{\dagger}\right\}\left(\mathcal{Q}_{5 z}^{b a \dagger}+\mathcal{Q}_{5 z}^{b a}+\mathcal{F}_{z}^{b a}{ }_{z}^{\dagger}\right) \\
\quad+\frac{1}{4}\left[\operatorname{Tr}\left\{\left(\mathcal{Q}_{5 z}+\mathcal{F}_{z}\right) U_{x}^{\dagger}\right\} \operatorname{Tr}\left\{U_{y}^{\dagger} U_{x}\right\}-\frac{1}{N_{c}} \operatorname{Tr}\left\{\left(\mathcal{Q}_{5 z}+\mathcal{F}_{z}\right) U_{y}^{\dagger}\right\}\right. \\
\left.\left.\quad+\operatorname{Tr}\left\{\left(\mathcal{Q}_{5 z}^{\dagger}+\mathcal{F}_{z}^{\dagger}\right) U_{x}\right\} \operatorname{Tr}\left\{U_{y} U_{x}^{\dagger}\right\}-\frac{1}{N_{c}} \operatorname{Tr}\left\{\left(\mathcal{Q}_{5 z}^{\dagger}+\mathcal{F}_{z}^{\dagger}\right) U_{y}\right\}\right]\right\} .
\end{aligned}
$$

So, if we neglect the quark-to-gluon diagrams and the mixing with the operator $\mathcal{Q}_{1}$ as is shown in eq. (6.49), then (F.13) does agree with the evolution equation calculated in refs. $[7,8]$.

\section{F.2 Diagrams quark-to-gluon for $\mathcal{Q}_{1 x}$ and $\mathcal{Q}_{5 x}$}

We calculate diagrams in figure 15 using again operator $Q_{i j}^{\alpha \beta}$ and then with the help of relations (F.1) and (F.2), we will obtain the results for operators $\mathcal{Q}_{5 x}$ and $\mathcal{Q}_{1 x}$ respectively.

The first diagram we will calculate is in figure $15 \mathrm{a}$

$$
\begin{aligned}
&\left\langle\operatorname{Tr}\left\{Q_{x}^{\alpha \beta} U_{y}^{\dagger}\right\}\right\rangle_{\text {Figure 15a }} \\
&=\left\langle\operatorname{Tr}\left\{g^{2} \int_{-\infty}^{+\infty} d z_{*} \int_{-\infty}^{z_{*}} d z_{*}^{\prime}\left[\infty p_{1}, z_{*}\right]_{x} t^{a} \psi^{\alpha}\left(z_{*}, x_{\perp}\right)\left[z_{*}, z_{*}^{\prime}\right]_{x}^{a b} \bar{\psi}^{\beta}\left(z_{*}^{\prime}, x_{\perp}\right) t^{b}\left[z_{*}^{\prime},-\infty p_{1}\right]_{x} U_{y}^{\dagger}\right\}\right\rangle \\
&=g^{2} \int_{-\infty}^{+\infty} d z_{*} \int_{-\infty}^{z_{*}} d z_{*}^{\prime} i g \frac{2}{s} \int_{z_{*}}^{+\infty} d \xi_{*}\left\langle\operatorname { T r } \left\{\left[\infty p_{1}, \xi_{*}\right]_{x} A_{\bullet}^{q}\left(\xi_{*}, x_{\perp}\right)\left[\xi_{*}, z_{*}\right]_{x} t^{a} \psi_{c l}^{\alpha}\left(z_{*}, x_{\perp}\right)\right.\right. \\
&\left.\left.\times\left[z_{*}, z_{*}^{\prime}\right]_{x}^{a b} \bar{\psi}_{q}^{\beta}\left(z_{*}^{\prime}, x_{\perp}\right) t^{b}\left[z_{*}^{\prime},-\infty p_{1}\right]_{x} U_{y}^{\dagger}\right\}\right\rangle
\end{aligned}
$$


To proceed, we should remember that the shock-wave has support only within the infinitesimal interval $\left[-\epsilon_{*}, \epsilon_{*}\right]$ and that, in a particular gauge, we can set to 1 the gauge links made of classical field with support outside the interval $\left[-\epsilon_{*}, \epsilon_{*}\right]$. Moreover, we can change the extremes of integration of the longitudinal variable $\xi^{+}$from $\left[z^{+}, \infty\right]$ to $[0, \infty]$ and those of $z^{\prime+}$ from $\left[-\infty, z^{+}\right]$to $[-\infty, 0]$ because the support of the quantum field gets up to zero. Thus, using propagator in eq. (6.55) we have

$$
\begin{aligned}
& \left\langle\operatorname{Tr}\left\{Q_{x}^{\alpha \beta} U_{y}^{\dagger}\right\}\right\rangle_{\text {Figure } 15 a} \\
& =g^{2} \int_{-\infty}^{+\infty} d z_{*} \int_{-\infty}^{z_{*}} d z_{*}^{\prime} i g \frac{2}{s} \int_{z_{*}}^{+\infty} d \xi_{*} \\
& \quad \times\left\langle\operatorname{Tr}\left\{t^{c}\left[\infty p_{1}, \xi_{*}\right]_{x} t^{a} \psi_{c l}^{\alpha}\left(z_{*}, x_{\perp}\right)\left[z_{*}, z_{*}^{\prime}\right]_{x}^{a b}\left\langle A_{*}^{q, c}\left(\xi_{*}, x_{\perp}\right) \bar{\psi}^{q, \beta}\left(z_{*}^{\prime}, x_{\perp}\right)\right\rangle t^{b}\left[z_{*}^{\prime},-\infty p_{1}\right]_{x} U_{y}^{\dagger}\right\}\right\rangle \\
& =-\frac{g^{4}}{s^{2}} \int_{-\infty}^{+\infty} d z_{*} d \omega_{*} \int_{-\infty}^{0} d z_{*}^{\prime} \int_{0}^{+\infty} d \xi_{*} \int_{0}^{+\infty} \frac{d \alpha}{\alpha^{3}} \int d^{2} z\left\langle x_{\perp}\left|e^{-i \frac{\hat{p}_{\perp}^{2}}{\alpha s} \xi_{*}} \hat{p}_{\nu}^{\perp}\right| z_{\perp}\right\rangle \\
& \quad \times \operatorname{Tr}\left\{t^{c}\left[\infty p_{1}, z_{*}\right]_{x} t^{a} \psi_{c l}^{\alpha}\left(z_{*}, x_{\perp}\right)\left[z_{*},-\infty p_{1}\right]_{x}^{a b}\left[\infty p_{1}, \omega_{*}\right]_{z}^{c d} \bar{\psi}_{c l}^{\beta^{\prime}}\left(\omega_{*}, z_{\perp}\right) t^{d}\left[\omega_{*},-\infty p_{1}\right]_{z} t^{b} U_{y}^{\dagger}\right. \\
& \left.\quad \times\left\langle z_{\perp}\left|\left[\gamma_{\perp}^{\nu}\left(\alpha \not p_{1}+\not p_{\perp}\right)\right]^{\beta^{\prime} \beta} e^{i \frac{\hat{p}_{\perp}^{2}}{\alpha s} z_{*}^{\prime}}\right| x_{\perp}\right\rangle\right\} \\
& =-\frac{g^{4}}{s^{2}} \int_{-\infty}^{+\infty} d z_{*} d \omega_{*} \int_{-\infty}^{0} d z_{*}^{\prime} \int_{0}^{+\infty} d \xi_{*} \int_{0}^{+\infty} \frac{d \alpha}{\alpha^{3}} \int d^{2} z\left\langle x_{\perp}\left|e^{-i \frac{\hat{p}_{\perp}^{2}}{\alpha s} \xi_{*}} \hat{p}_{\nu}^{\perp}\right| z_{\perp}\right\rangle \\
& \quad \times \operatorname{Tr}\left\{t^{c} U_{z} t^{b} U_{y}^{\dagger} t^{c} U_{x} t^{b}\left[-\infty p_{1}, z_{*}\right]\right]_{x} \psi_{c l}^{\alpha}\left(z_{*}, x_{\perp}\right) \bar{\psi}_{c l}^{\beta^{\prime}}\left(\omega_{*}, z_{\perp}\right)\left[\omega_{*}, \infty p_{1}\right]_{z} \\
& \quad \times\left\langle z_{\perp}\right|\left[\gamma_{\perp}^{\nu}\left(\alpha \not p_{1}+\not p_{\perp}\right)\right]^{\beta^{\prime} \beta} e^{\left.i \frac{\hat{p}_{\perp}^{2}}{\alpha s} z_{*}^{\prime}\left|x_{\perp}\right\rangle\right\}}
\end{aligned}
$$

Proceeding in the same way, with the exception that $d \xi_{*}$ starts from 0 and not from $z_{*}$, for diagram $15 \mathrm{~b}$ we have

$$
\begin{aligned}
\left\langle\operatorname{Tr}\left\{Q_{x}^{\alpha \beta} U_{y}^{\dagger}\right\}\right\rangle_{\text {Figure }} 15 b & \\
= & \left.\operatorname{Tr}\left\{g^{2} \int_{-\infty}^{+\infty} d z_{*} \int_{-\infty}^{z_{*}} d z_{*}^{\prime}\left[\infty p_{1}, z_{*}\right]_{x} t^{a} \psi^{\alpha}\left(z_{*}, x_{\perp}\right)\left[z_{*}, z_{*}^{\prime}\right]_{x}^{a b} \bar{\psi}^{\beta}\left(z_{*}^{\prime}, x_{\perp}\right) t^{b}\left[z_{*}^{\prime},-\infty p_{1}\right]_{x} U_{y}^{\dagger}\right\}\right\rangle \\
= & \frac{g^{4}}{s^{2}} \int_{-\infty}^{+\infty} d z_{*} d \omega_{*} \int_{-\infty}^{z_{*}} d z_{*}^{\prime} \int_{z_{*}}^{+\infty} d \xi_{*} \int_{0}^{+\infty} \frac{d \alpha}{\alpha^{3}} \int d^{2} z\left\langle y_{\perp}\left|e^{-i \frac{\hat{p}_{\perp}^{2}}{\alpha s} \xi_{*}} \hat{p}_{\nu}^{\perp}\right| z_{\perp}\right\rangle \\
& \times \operatorname{Tr}\left\{t^{c}\left[\infty p_{1}, z_{*}\right]_{x} t^{a} \psi_{c l}^{\alpha}\left(z_{*}, x_{\perp}\right)\left[z_{*},-\infty p_{1}\right]_{x}^{a b}\left[\infty p_{1}, \omega_{*}\right]_{z}^{c d} \bar{\psi}_{c l}^{\beta^{\prime}}\left(\omega_{*}, z_{\perp}\right) t^{d}\left[\omega_{*},-\infty p_{1}\right]_{z} t^{b} U_{y}^{\dagger}\right. \\
= & \left.\times\left\langle z_{\perp}\left|\left[\gamma_{\perp}^{\nu}\left(\alpha \not p_{1}+\not p_{\perp}\right)\right]^{\beta^{\prime} \beta} e^{i \frac{\hat{p}_{\perp}^{2}}{\alpha s} z_{*}^{\prime}}\right| x_{\perp}\right\rangle\right\} \\
g^{4} & \int_{-\infty}^{+\infty} d z_{*} d \omega_{*} \int_{-\infty}^{z_{*}} d z_{*}^{\prime} \int_{z_{*}}^{+\infty} d \xi_{*} \int_{0}^{+\infty} \frac{d \alpha}{\alpha^{3}} \int d^{2} z\left\langle y_{\perp}\left|e^{-i \frac{\hat{p}_{\perp}^{2}}{\alpha s} \xi_{*}} \hat{p}_{\nu}^{\perp}\right| z_{\perp}\right\rangle \\
& \times \operatorname{Tr}\left\{t^{c} U_{z} t^{b} U_{y}^{\dagger} t^{c} U_{x} t^{b}\left[-\infty p_{1}, z_{*}\right]_{x} \psi_{c l}^{\alpha}\left(z_{*}, x_{\perp}\right) \bar{\psi}_{c l}^{\beta^{\prime}}\left(\omega_{*}, z_{\perp}\right)\left[\omega_{*}, \infty p_{1}\right]_{z}\right. \\
& \left.\times\left\langle z_{\perp}\left|\left[\gamma_{\perp}^{\nu}\left(\alpha \not p_{1}+\not p_{\perp}\right)\right]^{\beta^{\prime} \beta} e^{i \frac{\hat{p}_{\perp}^{2}}{\alpha s} z_{*}^{\prime}}\right| x_{\perp}\right\rangle\right\} .
\end{aligned}
$$


The second self diagram, figure $15 \mathrm{c}$ is also calculated in a similar way. We have

$$
\begin{aligned}
\left\langle\operatorname{Tr}\left\{Q_{x}^{\alpha \beta} U_{y}^{\dagger}\right\}\right\rangle_{\text {Figure } 15 c} \\
=\left\langle\operatorname{Tr}\left\{g^{2} \int_{-\infty}^{+\infty} d z_{*} \int_{-\infty}^{z_{*}} d z_{*}^{\prime}\left[\infty p_{1}, z_{*}\right]_{x} t^{a} \psi^{\alpha}\left(z_{*}, x_{\perp}\right)\left[z_{*}, z_{*}^{\prime}\right]_{x}^{a b} \bar{\psi}^{\beta}\left(z_{*}^{\prime}, x_{\perp}\right) t^{b}\left[z_{*}^{\prime},-\infty p_{1}\right]_{x} U_{y}^{\dagger}\right\}\right\rangle \\
=\operatorname{Tr}\left\{g^{2} \int_{-\infty}^{+\infty} d z_{*} \int_{-\infty}^{z_{*}} d z_{*}^{\prime} t^{a} \psi_{q}^{\alpha}\left(z_{*}, x_{\perp}\right)\left[z_{*}, z_{*}^{\prime}\right]^{a b} \bar{\psi}_{c l}^{\beta}\left(z_{*}^{\prime}, x_{\perp}\right) t^{b}\right. \\
\left.\quad \times\left(i g \frac{2}{s}\right) \int_{-\infty}^{z_{*}^{\prime}} d \xi_{*}\left[z_{*}^{\prime}, \xi_{*}\right]_{x} A_{\bullet}^{q}\left(\xi_{*}, x_{\perp}\right) U_{y}^{\dagger}\right\}
\end{aligned}
$$

Now we use propagator (6.58) and obtain

$$
\begin{aligned}
& \left\langle\operatorname{Tr}\left\{Q_{x}^{\alpha \beta} U_{y}^{\dagger}\right\}\right\rangle_{\text {Figure } 15 c} \\
& =i g^{3} \frac{2}{s} \int_{-\infty}^{+\infty} d z_{*}^{\prime} \int_{z_{*}^{\prime}}^{+\infty} d z_{*} \int_{-\infty}^{z_{*}^{\prime}} d \xi_{*} \\
& \times \operatorname{Tr}\left\{t^{a}\left\langle\psi_{q}^{\alpha}\left(z_{*}, x_{\perp}\right) A_{\bullet}^{q, c}\left(\xi_{*}, x_{\perp}\right)\right\rangle\left[\infty p_{1}, z_{*}^{\prime}\right]_{x}^{a b} \bar{\psi}_{c l}^{\beta}\left(z_{*}^{\prime}, x_{\perp}\right) t^{b}\left[z_{*}^{\prime},-\infty p_{1}\right]_{x} t^{c} U_{y}^{\dagger}\right\} \\
& =-\frac{g^{4}}{s^{2}} \int_{-\infty}^{+\infty} d z_{*}^{\prime} d \omega_{*} \int_{0}^{+\infty} \frac{d \alpha}{\alpha^{3}} \int_{0}^{+\infty} d z_{*} \int_{-\infty}^{0} d \xi_{*} \int d^{2} z \\
& \times\left\langle x_{\perp}\left|e^{-i \frac{\hat{p}_{\perp}^{2}}{\alpha s} z_{*}}\left[\left(\alpha \not p_{1}+\not p_{\perp}\right) \gamma_{\perp}^{\nu}\right]^{\alpha \alpha^{\prime}}\right| z_{\perp}\right\rangle\left\langle z_{\perp}\left|p_{\nu}^{\perp} e^{i \frac{\hat{p}_{\perp}^{2}}{\alpha s} \xi_{*}}\right| x_{\perp}\right\rangle \\
& \times \operatorname{Tr}\left\{t^{a}\left[\infty p_{1}, \omega_{*}\right]_{z} t^{d} \psi^{\alpha^{\prime}}\left(\omega_{*}, z_{\perp}\right)\left[\omega_{*},-\infty p_{1}\right]_{z}^{d c}\left[\infty p_{1}, z_{*}^{\prime}\right]_{x}^{a b} \bar{\psi}^{\beta}\left(z_{*}^{\prime}, x_{\perp}\right) t^{b}\left[z_{*}^{\prime},-\infty p_{1}\right]_{x} t^{c} U_{y}^{\dagger}\right\} \\
& =-\frac{g^{4}}{s^{2}} \int_{-\infty}^{+\infty} d z_{*}^{\prime} d \omega_{*} \int_{0}^{+\infty} \frac{d \alpha}{\alpha^{3}} \int_{0}^{+\infty} d z_{*} \int_{-\infty}^{0} d \xi_{*} \int d^{2} z \\
& \times\left\langle x_{\perp}\left|e^{-i \frac{\hat{p}_{\perp}^{2}}{\alpha s} z_{*}}\left[\left(\alpha \not p_{1}+\not p_{\perp}\right) \gamma_{\perp}^{\nu}\right]^{\alpha \alpha^{\prime}}\right| z_{\perp}\right\rangle\left\langle z_{\perp}\left|p_{\nu}^{\perp} e^{i \frac{\hat{p}_{\perp}^{2}}{\alpha s} \xi_{*}}\right| x_{\perp}\right\rangle \\
& \times \operatorname{Tr}\left\{t^{a} U_{x} t^{c} U_{y}^{\dagger} t^{a} U_{z} t^{c}\left[-\infty p_{1}, \omega_{*}\right]_{z} \psi^{\alpha^{\prime}}\left(\omega_{*}, z_{\perp}\right) \bar{\psi}^{\beta}\left(z_{*}^{\prime}, x_{\perp}\right)\left[z_{*}^{\prime}, \infty p_{1}\right]_{x}\right\} .
\end{aligned}
$$

Diagram in figure $15 \mathrm{~d}$ is similar to $15 \mathrm{c}$, so we have

$$
\begin{aligned}
\left\langle\operatorname{Tr}\left\{Q_{x}^{\alpha \beta} U_{y}^{\dagger}\right\}\right\rangle_{\text {Figure 15d }} \\
=\left\langle\operatorname{Tr}\left\{g^{2} \int_{-\infty}^{+\infty} d z_{*} \int_{-\infty}^{z_{*}} d z_{*}^{\prime}\left[\infty p_{1}, z_{*}\right]_{x} t^{a} \psi^{\alpha}\left(z_{*}, x_{\perp}\right)\left[z_{*}, z_{*}^{\prime}\right]_{x}^{a b} \bar{\psi}^{\beta}\left(z_{*}^{\prime}, x_{\perp}\right) t^{b}\left[z_{*}^{\prime},-\infty p_{1}\right]_{x} U_{y}^{\dagger}\right\}\right\rangle \\
=\frac{g^{4}}{s^{2}} \int_{-\infty}^{+\infty} d z_{*}^{\prime} d \omega_{*} \int_{0}^{+\infty} \frac{d \alpha}{\alpha^{3}} \int_{0}^{+\infty} d z_{*} \int_{-\infty}^{0} d \xi_{*} \int d^{2} z \\
\quad \times\left\langle x_{\perp}\left|e^{-i \frac{\hat{p}_{\perp}^{2}}{\alpha s} z_{*}}\left[\left(\alpha \not p_{1}+\not p_{\perp}\right) \gamma_{\perp}^{\nu}\right]^{\alpha \alpha^{\prime}}\right| z_{\perp}\right\rangle\left\langle z_{\perp}\left|p_{\nu}^{\perp} e^{i \frac{\hat{p}_{\perp}^{2}}{\alpha s} \xi_{*}}\right| y_{\perp}\right\rangle \\
\quad \times \operatorname{Tr}\left\{t^{a} U_{x} t^{c} U_{y}^{\dagger} t^{a} U_{z} t^{c}\left[-\infty p_{1}, \omega_{*}\right]_{z} \psi^{\alpha^{\prime}}\left(\omega_{*}, z_{\perp}\right) \bar{\psi}^{\beta}\left(z_{*}^{\prime}, x_{\perp}\right)\left[z_{*}^{\prime}, \infty p_{1}\right]_{x}\right\} .
\end{aligned}
$$

The contribution of diagrams in figure 15 to the evolution of operators $\mathcal{Q}_{1}$ and $\mathcal{Q}_{5}$ defined in eqs. (4.9) and (4.10) respectively, can be obtained by taking the Dirac trace of the operator $Q_{i j}^{\alpha \beta}$ with $i \not p_{1}$ and $\gamma^{5} \not p_{1}$. 


\section{F.2.1 Quark-to-gluon diagrams for $\mathcal{Q}_{1 x}$}

Summing eqs. (F.15) and (F.16) and taking trace with $i \not p_{1}$ we have

$$
\begin{aligned}
& \left\langle\operatorname{Tr}\left\{\mathcal{Q}_{1 x} U_{y}^{\dagger}\right\}\right\rangle_{\text {Figure } 15 a+b} \\
& =\left\langle\operatorname{Tr} \operatorname{tr}\left\{i \not p_{1} Q_{x} U_{y}^{\dagger}\right\}\right\rangle_{\text {Figure } 15 a+b} \\
& =\frac{g^{4}}{s^{2}} \int_{-\infty}^{+\infty} d z_{*} d \omega_{*} \int_{-\infty}^{0} d z_{*}^{\prime} \int_{0}^{+\infty} d \xi_{*} \int_{0}^{+\infty} \frac{\pi \alpha}{\alpha^{3}} \int d^{2} z \\
& \times\left(\left\langle y_{\perp}\left|e^{-i \frac{\hat{p}_{\perp}^{2}}{\alpha s} \xi_{*}} \hat{p}_{\nu}^{\perp}\right| z_{\perp}\right\rangle-\left\langle x_{\perp}\left|e^{-i \frac{\hat{p}_{\perp}^{2}}{\alpha s} \xi_{*}} \hat{p}_{\nu}^{\perp}\right| z_{\perp}\right\rangle\right) \\
& \times \operatorname{Tr} \operatorname{tr}\left\{t^{c} U_{z} t^{b} U_{y}^{\dagger} t^{c} U_{x} t^{b}\left[-\infty p_{1}, z_{*}\right]_{x} i \not p_{1} \psi^{\alpha}\left(z_{*}, x_{\perp}\right) \bar{\psi}^{\beta^{\prime}}\left(\omega_{*}, z_{\perp}\right)\left[\omega_{*}, \infty p_{1}\right]_{z}\right. \\
& \left.\times\left\langle z_{\perp}\left|\gamma_{\perp}^{\nu}\left(\alpha \not p_{1}+\not p_{\perp}\right) e^{i \frac{\hat{p}_{\perp}^{2}}{\alpha s} z_{*}^{\prime}}\right| x_{\perp}\right\rangle\right\} \\
& =\frac{\alpha_{s}}{2 \pi^{2}} \int_{0}^{+\infty} \frac{d \alpha}{\alpha} g^{2} \int_{-\infty}^{+\infty} d z_{*} d \omega_{*} \int d^{2} z \\
& \times \operatorname{Tr} \operatorname{tr}\left\{t^{c} U_{z} t^{b} U_{y}^{\dagger} t^{c} U_{x} t^{b}\left[-\infty p_{1}, z_{*}\right]_{x} i \not p_{1} \psi^{\alpha}\left(z_{*}, x_{\perp}\right) \bar{\psi}^{\beta^{\prime}}\left(\omega_{*}, z_{\perp}\right)\left[\omega_{*}, \infty p_{1}\right]_{z}\right. \\
& \left.\times\left[\frac{(x-z, z-y)}{(x-z)_{\perp}^{2}(y-z)_{\perp}^{2}}+\frac{1}{(x-z)_{\perp}^{2}}+i \gamma^{5} \frac{(\vec{x}-\vec{z}) \times(\vec{y}-\vec{z})}{(x-z)_{\perp}^{2}(y-z)_{\perp}^{2}}\right]\right\}
\end{aligned}
$$

where in the last step we have integrated over the longitudinal variables $\xi^{+}$and $z^{\prime+}$ and performed the Fourier transform.

Summing up the next two diagrams, eqs. (F.18) and (F.19) we have

$$
\begin{aligned}
& \left\langle\operatorname{Tr}\left\{\mathcal{Q}_{1 x} U_{y}^{\dagger}\right\}\right\rangle_{\text {Figure } 15 c+d} \\
& =\left\langle\operatorname{Tr} \operatorname{tr}\left\{i \not p_{1} Q_{x} U_{y}^{\dagger}\right\}\right\rangle_{\text {Figure } 15 c+d} \\
& =\frac{g^{4}}{s^{2}} \int_{-\infty}^{+\infty} d z_{*}^{\prime} d \omega_{*} \int_{0}^{+\infty} \frac{d \alpha}{\alpha^{3}} \int_{0}^{+\infty} d z_{*} \int_{-\infty}^{0} d \xi_{*} \int d^{2} z \\
& \quad \times \operatorname{tr}\left\{\left\langle x_{\perp}\left|e^{-i \frac{\hat{p}_{\perp}^{2}}{\alpha s} z_{*}} i \not p_{1}\left[\left(\alpha \not p_{1}+\not p_{\perp}\right) \gamma_{\perp}^{\nu}\right]\right| z_{\perp}\right\rangle\left(\left\langle z_{\perp}\left|p_{\nu}^{\perp} e^{i \frac{\hat{p}_{\perp}^{2}}{\alpha s} \xi_{*}}\right| y_{\perp}\right\rangle-\left\langle z_{\perp}\left|p_{\nu}^{\perp} e^{i \frac{\hat{p}_{\perp}^{2}}{\alpha s} \xi_{*}}\right| x_{\perp}\right\rangle\right)\right. \\
& \left.\quad \times \operatorname{Tr}\left\{t^{a} U_{x} t^{c} U_{y}^{\dagger} t^{a} U_{z} t^{c}\left[-\infty p_{1}, \omega_{*}\right] \psi\left(\omega_{*}, z_{\perp}\right) \bar{\psi}\left(z_{*}^{\prime}, x_{\perp}\right)\left[z_{*}^{\prime}, \infty p_{1}\right] x\right\}\right\} \\
& =\frac{\alpha_{s}}{2 \pi^{2}} \int_{0}^{+\infty} \frac{d \alpha}{\alpha} g^{2} \int_{-\infty}^{+\infty} d z_{*}^{\prime} d \omega_{*} \int d^{2} z \\
& \times \operatorname{tr}\left\{\left[\frac{(x-z, z-y)}{(x-z)_{\perp}^{2}(y-z)_{\perp}^{2}}+\frac{1}{(x-z)_{\perp}^{2}}-i \gamma^{5} \frac{(\vec{x}-\vec{z}) \times(\vec{y}-\vec{z})}{(x-z)_{\perp}^{2}(y-z)_{\perp}^{2}}\right]\right. \\
& \left.\quad \times \operatorname{Tr}\left\{t^{a} U_{x} t^{c} U_{y}^{\dagger} t^{a} U_{z} t^{c}\left[-\infty p_{1}, \omega_{*}\right]_{z} i \not p_{1} \psi\left(\omega_{*}, z_{\perp}\right) \bar{\psi}\left(z_{*}^{\prime}, x_{\perp}\right)\left[z_{*}^{\prime}, \infty p_{1}\right]_{x}\right\}\right\} . \quad(\mathrm{F} .21)
\end{aligned}
$$


We use definition of the operators (6.59) and (6.61), and the sum of eq. (F.20) and (F.21) traced with $i \not p_{1}$ is

$$
\begin{aligned}
\langle\operatorname{Tr} & \left.\left\{\mathcal{Q}_{1 x} U_{y}^{\dagger}\right\}\right\rangle_{\text {Figure } 15} \\
= & -\frac{\alpha_{s}}{2 \pi^{2}} \int_{0}^{+\infty} \frac{d \alpha}{\alpha} \int d^{2} z\left\{\left[\frac{(x-z, z-y)}{(x-z)_{\perp}^{2}(y-z)_{\perp}^{2}}+\frac{1}{(x-z)_{\perp}^{2}}\right]\right. \\
& \times\left(\operatorname{Tr}\left\{t^{a} U_{z} t^{b} U_{y}^{\dagger} t^{a} U_{x} t^{b} \mathcal{X}_{1 z x}^{\dagger}\right\}+\operatorname{Tr}\left\{t^{a} U_{x} t^{b} U_{y}^{\dagger} t^{a} U_{z} t^{b} \mathcal{X}_{1 x z}^{\dagger}\right\}\right) \\
& \left.-\frac{(\vec{x}-\vec{z}) \times(\vec{y}-\vec{z})}{(x-z)_{\perp}^{2}(y-z)_{\perp}^{2}}\left(\operatorname{Tr}\left\{t^{a} U_{x} t^{b} U_{y}^{\dagger} t^{a} U_{z} t^{b} \mathcal{X}_{5 x z}^{\dagger}\right\}-\operatorname{Tr}\left\{t^{a} U_{z} t^{b} U_{y}^{\dagger} t^{a} U_{x} t^{b} \mathcal{X}_{5 z x}^{\dagger}\right\}\right)\right\}
\end{aligned}
$$

The color trace in eq. (F.20) can be simplified. For example, we have

$$
\begin{aligned}
\operatorname{Tr}\left\{t^{a} U_{z} t^{b} U_{y}^{\dagger} t^{a} U_{x} t^{b} \mathcal{X}_{1 z x}^{\dagger}\right\} & \\
= & \frac{1}{4} \operatorname{Tr}\left\{U_{x} U_{y}^{\dagger} U_{z} \mathcal{X}_{1 z x}^{\dagger}\right\}-\frac{1}{4 N_{c}} \operatorname{Tr}\left\{U_{y}^{\dagger} U_{z}\right\} \operatorname{Tr}\left\{U_{x} \mathcal{X}_{1 z x}^{\dagger}\right\} \\
& \quad-\frac{1}{4 N_{c}} \operatorname{Tr}\left\{U_{y}^{\dagger} U_{x}\right\} \operatorname{Tr}\left\{\mathcal{X}_{1 z x}^{\dagger} U_{z}\right\}-\frac{1}{4 N_{c}^{2}} \operatorname{Tr}\left\{U_{z} U_{y}^{\dagger} U_{x} \mathcal{X}_{1 z x}^{\dagger}\right\}
\end{aligned}
$$

Note also that the products like $\mathcal{X}_{1 z x}^{\dagger} U_{z}$ or $U_{x} \mathcal{X}_{1 z x}^{\dagger}$ may modify the operator $\mathcal{X}_{1 z x}^{\dagger}$ to

$$
\begin{aligned}
\operatorname{Tr}\left\{\mathcal{X}_{1 z x}^{\dagger} U_{z}\right\} & =-g^{2} \int_{-\infty}^{+\infty} d z_{*} d \omega_{*} \operatorname{Tr}\left\{\left[-\infty p_{1}, z_{*}\right]_{x} \operatorname{tr}\left\{i \not p_{1} \psi\left(z_{*}, x_{\perp}\right) \bar{\psi}\left(\omega_{*}, z_{\perp}\right)\right\}\left[\omega_{*},-\infty p_{1}\right]_{z}\right\} \\
& =-\mathcal{H}_{1}^{-}\left(x_{\perp}, z_{\perp}\right)
\end{aligned}
$$

and

$$
\begin{aligned}
\operatorname{Tr}\left\{U_{x} \mathcal{X}_{1 z x}^{\dagger}\right\} & =-g^{2} \int_{-\infty}^{+\infty} d z_{*} d \omega_{*} \operatorname{Tr}\left\{\left[\infty p_{1}, z_{*}\right]_{x} \operatorname{tr}\left\{i \not p_{1} \psi\left(z_{*}, x_{\perp}\right) \bar{\psi}\left(\omega_{*}, z_{\perp}\right)\right\}\left[\omega_{*}, \infty p_{1}\right]_{z}\right\} \\
& =-\mathcal{H}_{1}^{+}\left(x_{\perp}, z_{\perp}\right)
\end{aligned}
$$

where we used definition of operators (6.65) and (6.63) respectively. It is also easy to find the operators $\mathcal{H}_{1 x z}^{-\dagger}=-\operatorname{Tr}\left\{U_{z}^{\dagger} \mathcal{X}_{1 z x}\right\}=-\mathcal{H}_{1 z x}^{-}$, and similarly, $\mathcal{H}_{1 x z}^{+\dagger}=-\mathcal{H}_{1 z x}^{+}$. With $\mathcal{X}_{5 x z}$, instead, we have

$$
\begin{aligned}
\operatorname{Tr}\left\{\mathcal{X}_{5 z x}^{\dagger} U_{z}\right\} & =g^{2} \int_{-\infty}^{+\infty} d z_{*} d \omega_{*} \operatorname{Tr}\left\{\left[-\infty p_{1}, z_{*}\right]_{x} \operatorname{tr}\left\{i \not p_{1} \psi\left(z_{*}, x_{\perp}\right) \bar{\psi}\left(\omega_{*}, z_{\perp}\right)\right\}\left[\omega_{*},-\infty p_{1}\right]_{z}\right\} \\
& =\mathcal{H}_{5}^{-}\left(x_{\perp}, z_{\perp}\right)
\end{aligned}
$$

and

$$
\begin{aligned}
\operatorname{Tr}\left\{U_{x} \mathcal{X}_{5 z x}^{\dagger}\right\} & =g^{2} \int_{-\infty}^{+\infty} d z_{*} d \omega_{*} \operatorname{Tr}\left\{\left[\infty p_{1}, z_{*}\right]_{x} \operatorname{tr}\left\{i \not p_{1} \psi\left(z_{*}, x_{\perp}\right) \bar{\psi}\left(\omega_{*}, z_{\perp}\right)\right\}\left[\omega_{*}, \infty p_{1}\right]_{z}\right\} \\
& =\mathcal{H}_{5}^{+}\left(x_{\perp}, z_{\perp}\right)
\end{aligned}
$$

where we used definition of operators (6.66) and (6.64) respectively. We also have $\mathcal{H}_{5 x z}^{-\dagger}=$ $\operatorname{Tr}\left\{\mathcal{X}_{5 z x} U_{z}^{\dagger}\right\}=\mathcal{H}_{5 z x}^{-}$and similarly we get $\mathcal{H}_{5 x z}^{+\dagger}=\mathcal{H}_{5 z x}^{+}$

Evolution of operator $\operatorname{Tr}\left\{\hat{\mathcal{Q}}_{1 x} \hat{U}_{y}^{\dagger}\right\}$, when diagrams in figure 15 are taken into account, introduces new operators, $\mathcal{X}_{1 x y}, \mathcal{X}_{5 x y}, \mathcal{H}_{1 x y}^{-}, \mathcal{H}_{5 x y}^{-}, \mathcal{H}_{1 x y}^{+}, \mathcal{H}_{5 x y}^{+}$, which have never been considered before in the study of spin dynamics at small- $x$. 


\section{F.2.2 Quark-to-gluon diagrams for $\mathcal{Q}_{5 x}$}

Summing eqs. (F.15) and (F.16) and taking trace with $\gamma^{5} \not p_{1}$, we have

$$
\begin{aligned}
\left\langle\operatorname{Tr}\left\{\mathcal{Q}_{5 x} U_{y}^{\dagger}\right\}\right\rangle_{\text {Figure } 15 a+b} \\
=\left\langle\operatorname{Trtr}\left\{\gamma^{5} \not p_{1} Q_{x} U_{y}^{\dagger}\right\}\right\rangle_{\text {Figure } 15 a+b} \\
=\frac{g^{4}}{s^{2}} \int_{-\infty}^{+\infty} d z_{*} d \omega_{*} \int_{-\infty}^{0} d z_{*}^{\prime} \int_{0}^{+\infty} d \xi_{*} \int_{0}^{+\infty} \frac{d \alpha}{\alpha^{3}} \int d^{2} z \\
\quad \times\left(\left\langle y_{\perp}\left|e^{-i \frac{\hat{p}_{\perp}^{2}}{\alpha s} \xi_{*}} \hat{p}_{\nu}^{\perp}\right| z_{\perp}\right\rangle-\left\langle x_{\perp}\left|e^{-i \frac{\hat{p}_{\perp}^{2}}{\alpha s} \xi_{*}} \hat{p}_{\nu}^{\perp}\right| z_{\perp}\right\rangle\right) \operatorname{Tr} \operatorname{tr}\left\{t^{a} U_{z} t^{b} U_{y}^{\dagger} t^{a} U_{x} t^{b}\right. \\
\left.\quad \times\left[-\infty p_{1}, z_{*}\right]_{x} \gamma^{5} \not p_{1} \psi_{c l}\left(z_{*}, x_{\perp}\right) \bar{\psi}_{c l}\left(\omega_{*}, z_{\perp}\right)\left[\omega_{*}, \infty p_{1}\right]_{z}\left\langle z_{\perp}\left|\gamma_{\perp}^{\nu}\left(\alpha \not p_{1}+\not p_{\perp}\right) e^{i \frac{\hat{p}_{\perp}^{2}}{\alpha s} z_{*}^{\prime}}\right| x_{\perp}\right\rangle\right\} \\
=\frac{\alpha_{s}}{2 \pi^{2}} g^{2} \int_{-\infty}^{+\infty} d z_{*} d \omega_{*} \int_{0}^{+\infty} \frac{d \alpha}{\alpha} \int d^{2} z \\
\quad \times \operatorname{Tr} \operatorname{tr}\left\{t^{a} U_{z} t^{b} U_{y}^{\dagger} t^{a} U_{x} t^{b}\left[-\infty p_{1}, z_{*}\right]_{x} \psi_{c l}\left(z_{*}, x_{\perp}\right) \bar{\psi}_{c l}\left(\omega_{*}, z_{\perp}\right)\left[\omega_{*}, \infty p_{1}\right]_{z}\right. \\
\left.\quad \times\left[\gamma^{5} \not p_{1}\left(\frac{(x-z, z-y)}{(x-z)_{\perp}^{2}(y-z)_{\perp}^{2}}+\frac{1}{(x-z)_{\perp}^{2}}\right)+i \not p_{1} \frac{(\vec{x}-\vec{z}) \times(\vec{y}-\vec{z})}{(x-z)_{\perp}^{2}(y-z)_{\perp}^{2}}\right]\right\} \quad(\mathrm{F} .28)
\end{aligned}
$$

Now we sum eqs. (F.18) and (F.19) and trace them with $\gamma^{5} \not p_{1}$ we have

$$
\begin{aligned}
\left\langle\operatorname{Tr}\left\{\mathcal{Q}_{5 x} U_{y}^{\dagger}\right\}\right\rangle_{\text {Figure } 15 c+d} \\
=\left\langle\operatorname{Tr} \operatorname{tr}\left\{\gamma^{5} \not p_{1} Q_{x} U_{y}^{\dagger}\right\}\right\rangle_{\text {Figure } 15 c+d} \\
=\frac{g^{4}}{s^{2}} \int_{-\infty}^{+\infty} d z_{*}^{\prime} d \omega_{*} \int_{0}^{+\infty} \frac{d \alpha}{\alpha^{3}} \int_{0}^{+\infty} d z_{*} \int_{-\infty}^{0} d \xi_{*} \int d^{2} z \\
\quad \times \operatorname{tr}\left\{\left\langle x_{\perp}\left|e^{-i \frac{\hat{p}_{\perp}^{2}}{\alpha s} z_{*}} \gamma^{5} \not p_{1}\left[\left(\alpha \not p_{1}+\not p_{\perp}\right) \gamma_{\perp}^{\nu}\right]\right| z_{\perp}\right\rangle\left(\left\langle z_{\perp}\left|p_{\nu}^{\perp} e^{i \frac{\hat{p}_{\perp}^{2}}{\alpha s} \xi_{*}}\right| y_{\perp}\right\rangle-\left\langle z_{\perp}\left|p_{\nu}^{\perp} e^{i \frac{\hat{p}_{\perp}^{2}}{\alpha s} \xi_{*}}\right| x_{\perp}\right\rangle\right)\right. \\
\left.\quad \times \operatorname{Tr}\left\{t^{a} U_{x} t^{b} U_{y}^{\dagger} t^{a} U_{z} t^{b}\left[-\infty p_{1}, \omega_{*}\right] \psi\left(\omega_{*}, z_{\perp}\right) \bar{\psi}\left(z_{*}^{\prime}, x_{\perp}\right)\left[z_{*}^{\prime}, \infty p_{1}\right]_{x}\right\}\right\} \\
=\frac{\alpha_{s}}{2 \pi^{2}} \int_{0}^{+\infty} \frac{d \alpha}{\alpha} g^{2} \int_{-\infty}^{+\infty} d z_{*}^{\prime} d \omega_{*} \int d^{2} z \\
\quad \times \operatorname{tr}\left\{\left[\gamma^{5} \not p_{1}\left(\frac{(x-z, z-y)}{(x-z)_{\perp}^{2}(y-z)_{\perp}^{2}}+\frac{1}{(x-z)_{\perp}^{2}}\right)-i \not p_{1} \frac{(\vec{x}-\vec{z}) \times(\vec{y}-\vec{z})}{(x-z)_{\perp}^{2}(y-z)_{\perp}^{2}}\right]\right. \\
\left.\quad \times \operatorname{Tr}\left\{t^{a} U_{x} t^{b} U_{y}^{\dagger} t^{a} U_{z} t^{b}\left[-\infty p_{1}, \omega_{*}\right] \psi\left(\omega_{*}, z_{\perp}\right) \bar{\psi}\left(z_{*}^{\prime}, x_{\perp}\right)\left[z_{*}^{\prime}, \infty p_{1}\right]_{x}\right\}\right\} .
\end{aligned}
$$

We can now sum eqs. (F.28) and (F.29) and obtain

$\left\langle\operatorname{Tr}\left\{\mathcal{Q}_{5 x} U_{y}^{\dagger}\right\}\right\rangle_{\text {Figure } 15}$

$$
\begin{aligned}
= & \frac{\alpha_{s}}{2 \pi^{2}} \int_{0}^{+\infty} \frac{d \alpha}{\alpha} \int d^{2} z\left\{\left[\frac{(x-z, z-y)}{(x-z)_{\perp}^{2}(y-z)_{\perp}^{2}}+\frac{1}{(x-z)_{\perp}^{2}}\right]\right. \\
& \times\left(\operatorname{Tr}\left\{t^{a} U_{z} t^{b} U_{y}^{\dagger} t^{a} U_{x} t^{b} \mathcal{X}_{5 z x}^{\dagger}\right\}+\operatorname{Tr}\left\{t^{a} U_{x} t^{b} U_{y}^{\dagger} t^{a} U_{z} t^{b} \mathcal{X}_{5 x z}^{\dagger}\right\}\right) \\
& \left.+\frac{(\vec{x}-\vec{z}) \times(\vec{y}-\vec{z})}{(x-z)_{\perp}^{2}(y-z)_{\perp}^{2}}\left(\operatorname{Tr}\left\{t^{a} U_{x} t^{b} U_{y}^{\dagger} t^{a} U_{z} t^{b} \mathcal{X}_{1 x z}^{\dagger}\right\}-\operatorname{Tr}\left\{t^{a} U_{z} t^{b} U_{y}^{\dagger} t^{a} U_{x} t^{b} \mathcal{X}_{1 z x}^{\dagger}\right\}\right)\right\}
\end{aligned}
$$

where we used the operators $\mathcal{X}_{1 x y}$ and $\mathcal{X}_{5 x y}$ defined in eqs. (6.59) and (6.61) respectively. 


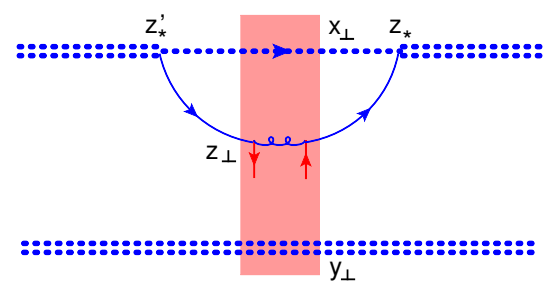

a)

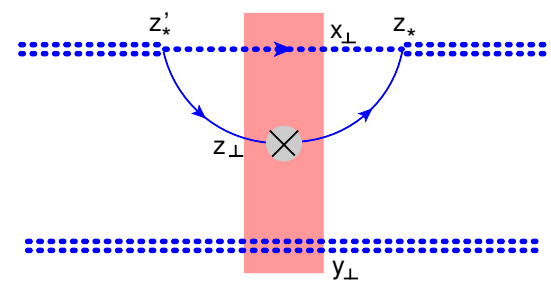

b)

Figure 16. Diagrams for the quark operators in the adjoint representation eqs. (6.44)-(6.47). Double dotted lines represent Wilson lines in the adjoint representation.

\section{F.3 Evolution equation with operators in the adjoint representation}

Let $T^{a}$ be the matrix in the adjoint representation, and $\operatorname{Tr}_{\mathrm{a}}$ the trace in the adjoint representation. From result (6.49) we can easily deduce

$$
\begin{aligned}
\left\langle\operatorname{Tr}_{\mathrm{a}}\left\{\mathcal{F}_{x} U_{y}^{\dagger}\right\}\right\rangle_{\text {Figures }} 9+10 & \\
= & \frac{\alpha_{s}}{\pi^{2}} \operatorname{Tr}_{\mathrm{a}}\left\{U_{x} T^{a} U_{y}^{\dagger} T^{b}\right\} \int_{0}^{+\infty} \frac{d \alpha}{\alpha} \int d^{2} z \\
\times & \left\{\left[-\frac{(\vec{x}-\vec{z}) \times(\vec{z}-\vec{y})}{(x-z)_{\perp}^{2}(y-z)_{\perp}^{2}}\left(\mathcal{Q}_{1 z}^{b a}-\mathcal{Q}_{1 z}^{b a \dagger}\right)\right.\right. \\
& \left.+\left(\frac{(x-z, z-y)}{(x-z)_{\perp}^{2}(y-z)_{\perp}^{2}}+\frac{1}{(x-z)_{\perp}^{2}}\right)\left(\mathcal{Q}_{5 z}^{b a}+\mathcal{Q}_{5 z}^{b a \dagger}+\mathcal{F}_{z}^{b a}\right)\right] \\
& \left.+4 \pi^{2} \int d^{2} q_{1} \frac{e^{i\left(q_{1}, y-z\right)}-e^{i\left(q_{1}, x-z\right)}}{q_{1 \perp}^{2}} \delta^{(2)}(z-x) \mathcal{F}_{z}^{b a}\right\}
\end{aligned}
$$

Let us consider the evolution of $\mathcal{Q}_{1 x}^{a b} U_{y}^{b a}$ and $\mathcal{Q}_{5 x}^{a b} U_{y}^{b a}$ starting with the diagrams with quark in the background shock-wave given in figure 16a

$$
\begin{aligned}
& \left\langle\mathcal{Q}_{5}^{a b^{\dagger}}\left(x_{\perp}\right)\right\rangle_{\text {Figure } 16 a} \\
& =\left\langle g^{2} \int_{-\infty}^{+\infty} d z_{1 *} \int_{-\infty}^{z_{1 *}} d z_{2 *}\left[-\infty p_{1}, z_{2 *}\right]_{x}^{b c} \bar{\psi}\left(z_{2 *}\right) \gamma^{5} \not p_{1} t^{c}\left[z_{2 *}, z_{1 *}\right]_{x} t^{d} \psi\left(z_{1 *}\right)\left[z_{1 *}, \infty p_{1}\right]_{x}^{d a}\right\rangle \\
& =-g^{2} \int_{0}^{+\infty} d z_{1 *} \int_{-\infty}^{0} d z_{2 *}\left(t^{b} U_{x}^{\dagger} t^{a}\right)_{i j} \operatorname{tr}\left\{\gamma^{5} \not p_{1}\left\langle\psi_{j}\left(z_{1 *}, x_{\perp}\right) \bar{\psi}_{i}\left(z_{2 *}, x_{\perp}\right)\right\rangle\right\}
\end{aligned}
$$

Using the quark propagator in the background of quark filed given in eq. (3.7) we obtain

$$
\begin{aligned}
\left\langle\mathcal{Q}_{5}^{a b^{\dagger}}\left(x_{\perp}\right)\right\rangle_{\text {Figure }} 16 a & \\
=- & g^{4} \int_{0}^{+\infty} d z_{1 *} \int_{-\infty}^{0} d z_{2 *} \int_{0}^{+\infty} \frac{d \alpha}{2 \alpha^{3} s^{2}} \int_{z_{2 *}}^{z_{1 *}} d z_{*} \int_{z_{2 *}}^{z_{1 *}} d z_{*}^{\prime} \int d^{2} z\left(t^{b} U_{x}^{\dagger} t^{a}\right)_{i j} \\
\times & \operatorname{tr}\left\{\gamma^{5} \not p_{1}\left\langle x_{\perp}\left|e^{-i \frac{p_{\perp}^{2}}{\alpha s} z_{1 *}}\left(\alpha \not p_{1}+\not p_{\perp}\right)\right| z_{\perp}\right\rangle\left[\infty p_{1}, z_{*}\right]_{z} \gamma_{\perp}^{\mu} t^{c} \psi\left(z_{*}, z_{\perp}\right)\left[z_{*}, z_{*}^{\prime}\right]_{z}^{c d}\right. \\
& \left.\times \bar{\psi}\left(z_{*}^{\prime}, z_{\perp}\right) t^{d} \gamma_{\mu}^{\perp}\left[z_{*}^{\prime},-\infty p_{1}\right]_{z}\left\langle z_{\perp}\left|\left(\alpha \not p_{1}+\not p_{\perp}\right) e^{i \frac{p_{\perp}^{2}}{\alpha s} z_{2 *}}\right| x_{\perp}\right\rangle\right\}_{j i}
\end{aligned}
$$


The integration over the longitudinal directions $z_{1}^{+}$and $z_{2}^{+}$yields

$$
\begin{aligned}
\left\langle\mathcal{Q}_{5}^{a b^{\dagger}}\left(x_{\perp}\right)\right\rangle_{\text {Figure } 16 a} & \\
= & -g^{4} \int_{0}^{+\infty} \frac{d \alpha}{2 \alpha^{3} s^{2}} \int d^{2} z \int_{z_{2 *}}^{z_{1 *}} d z_{*} \int_{z_{2 *}}^{z_{1 *}} d z_{*}^{\prime}\left(t^{b} U_{x}^{\dagger} t^{a}\right)_{i j} \operatorname{tr}\left\{\gamma^{5} \not p_{1}\left\langle x_{\perp}\left|\frac{-i \alpha s \not p_{\perp}}{p_{\perp}^{2}}\right| z_{\perp}\right\rangle\right. \\
& \left.\times\left[\infty p_{1}, z_{*}\right]_{z} \gamma_{\perp}^{\mu} t^{c} \psi\left(z_{*}\right)\left[z_{*}, z_{*}^{\prime}\right]_{z}^{c d} \bar{\psi}\left(z_{*}^{\prime}\right) t^{d} \gamma_{\mu}^{\perp}\left[z_{*}^{\prime},-\infty p_{1}\right]_{z}\left\langle z_{\perp}\left|\frac{-i \alpha s \not p_{\perp}}{p_{\perp}^{2}}\right| x_{\perp}\right\rangle\right\}_{j i}
\end{aligned}
$$

We can now perform the Fourier transform in coordinate space and arrive at

$$
\begin{aligned}
\left\langle\mathcal{Q}_{5}^{a b^{\dagger}}\left(x_{\perp}\right)\right\rangle_{\text {Figure 16a }} & \\
= & g^{2} \int_{0}^{+\infty} \frac{d \alpha}{2 \alpha} \int d^{2} z\left(t^{b} U_{x}^{\dagger} t^{a}\right)_{i j} \operatorname{tr}\left\{\gamma^{5} \not p_{1} \frac{i(\not x-\not)_{\perp}}{2 \pi(x-z)_{\perp}^{2}} g^{2} \int_{-\infty}^{+\infty} d z_{*} \int_{-\infty}^{z_{*}} d z_{*}^{\prime}\right. \\
& \left.\times\left[\infty p_{1}, z_{*}\right]_{z} \gamma_{\perp}^{\mu} t^{c} \psi\left(z_{*}\right)\left[z_{*}, z_{*}^{\prime}\right]_{z}^{c d} \bar{\psi}\left(z_{*}^{\prime}\right) t^{d} \gamma_{\mu}^{\perp}\left[z_{*}^{\prime}, z_{*}\right]_{z} \frac{i(\not-\not x)_{\perp}}{2 \pi(x-z)_{\perp}^{2}}\right\}_{j i}
\end{aligned}
$$

performing the trace over the Dirac gamma-matrices and using $(\not x-\not)_{\perp}(\not x-\not)_{\perp}=-(x-z)_{\perp}^{2}$ we obtain

$$
\begin{aligned}
\left\langle\mathcal{Q}_{5}^{a b^{\dagger}}\left(x_{\perp}\right)\right\rangle_{\text {Figure }} 16 a & \\
= & -\frac{g^{2}}{8 \pi^{3}} \int_{0}^{+\infty} \frac{d \alpha}{\alpha} \int d^{2} z \frac{1}{(x-z)_{\perp}^{2}}\left(t^{b} U_{x}^{\dagger} t^{a}\right)_{i j} g^{2} \int_{-\infty}^{+\infty} d z_{*} \int_{-\infty}^{z_{*}} d z_{*}^{\prime} \\
& \times\left(\left[\infty p_{1}, z_{*}\right]_{z} t^{c} \operatorname{tr}\left\{\gamma^{5} \not p_{1} \psi\left(z_{*}\right)\left[z_{*}, z_{*}^{\prime}\right]^{c d} \bar{\psi}\left(z_{*}^{\prime}\right) t^{d}\left[z_{*}^{\prime},-\infty p_{1}\right]_{z}\right\}\right)_{j i} \\
= & -\frac{\alpha_{s}}{2 \pi^{2}} \int_{0}^{+\infty} \frac{d \alpha}{\alpha} \int d^{2} z \frac{1}{(x-z)_{\perp}^{2}}\left(t^{b} U_{x}^{\dagger} t^{a}\right)_{i j} \mathcal{Q}_{5 j i}\left(z_{\perp}\right)
\end{aligned}
$$

where in the last step we inserted the $\mathcal{Q}_{5 x}$ definition eq. (4.10). Until now the Wilson line operator $U_{y}^{a b}$ was only a spectator, inserting it back we finally obtain

$$
\begin{aligned}
\left\langle U_{y}^{a b}\right. & \left.\mathcal{Q}_{5 x}^{a b}\right\rangle_{\text {Figure } 16 a} \\
& =-\frac{\alpha_{s}}{2 \pi^{2}} \int_{0}^{+\infty} \frac{d \alpha}{\alpha} \int d^{2} z \frac{1}{(x-z)_{\perp}^{2}} U_{y}^{a b} \operatorname{Tr}\left\{t^{b} U_{x}^{\dagger} t^{a} \mathcal{Q}_{5 z}\right\} \\
& =-\frac{\alpha_{s}}{2 \pi^{2}} \int_{0}^{+\infty} \frac{d \alpha}{\alpha} \int d^{2} z \frac{1}{(x-z)_{\perp}^{2}} \frac{1}{2}\left[\operatorname{Tr}\left\{U_{y} U_{x}^{\dagger}\right\} \operatorname{Tr}\left\{\mathcal{Q}_{5 z} U_{y}^{\dagger}\right\}-\frac{1}{N_{c}} \operatorname{Tr}\left\{U_{x}^{\dagger} \mathcal{Q}_{5 z}\right\}\right]
\end{aligned}
$$

Notice that on the 1.h.s. of eq. (F.37) we have trace in the adjoint representation and operator $\mathcal{Q}^{a b}$ defined in (6.45), while on the r.h.s. we have trace over the fundamental representation with operator $\mathcal{Q}_{5 x}$ defined in eq. (4.10). Following similar steps, the result of diagram in figure 16a for the $\mathcal{Q}_{1 x}^{a b}{ }^{\dagger}$ operator is

$$
\begin{aligned}
& \left\langle\mathcal{Q}_{1 x}^{a b}\right\rangle_{\text {Figure } 16 a} \\
& =\left\langle-g^{2} \int_{-\infty}^{+\infty} d z_{1 *} \int_{-\infty}^{z_{1 *}} d z_{2 *}\left[-\infty p_{1}, z_{2 *}\right]_{x}^{b c} \bar{\psi}\left(z_{2 *}\right) i p_{1} t^{c}\left[z_{2 *}, z_{1 *}\right]_{x} t^{d} \psi\left(z_{1 *}\right)\left[z_{1 *}, \infty p_{1}\right]_{x}^{d a}\right\rangle \\
& =g^{2} \int_{0}^{+\infty} d z_{1 *} \int_{-\infty}^{0} d z_{2 *}\left(t^{b} U_{x}^{\dagger} t^{a}\right)_{i j} \operatorname{tr}\left\{i \not p_{1}\left\langle\psi_{j}\left(z_{1 *}, x_{\perp}\right) \bar{\psi}_{i}\left(z_{2 *}, x_{\perp}\right)\right\}\right\rangle \\
& =-\frac{\alpha_{s}}{2 \pi^{2}} \int_{0}^{+\infty} \frac{d \alpha}{\alpha} \int d^{2} z \frac{1}{(x-z)_{\perp}^{2}} \operatorname{Tr}\left\{t^{b} U_{x}^{\dagger} t^{a} \mathcal{Q}_{1 z}\right\}
\end{aligned}
$$


Thus we have

$$
\begin{aligned}
& \left\langle U_{y}^{a b} \mathcal{Q}_{1 x}^{a b{ }^{\dagger}}\right\rangle_{\text {Figure 16a }} \\
& \quad=-\frac{\alpha_{s}}{4 \pi^{2}} \int_{0}^{+\infty} \frac{d \alpha}{\alpha} \int d^{2} z \frac{1}{(x-z)_{\perp}^{2}}\left[\operatorname{Tr}\left\{U_{y} U_{x}^{\dagger}\right\} \operatorname{Tr}\left\{\mathcal{Q}_{1 z} U_{y}^{\dagger}\right\}-\frac{1}{N_{c}} \operatorname{Tr}\left\{U_{x}^{\dagger} \mathcal{Q}_{1 z}\right\}\right]
\end{aligned}
$$

We now consider diagram in figure $16 \mathrm{~b}$ which is the one with the gluon sub-eikonal correction $\epsilon^{i j} F_{i j}$ in the quark propagator. Let us start again with operator $\mathcal{Q}_{5 x}^{a b}$

$$
\begin{aligned}
& \left.\left\langle\mathcal{Q}_{5 x}^{a b}\right\rangle^{\dagger}\right\rangle_{\text {Figure }} 16 b \\
& \quad=\left\langle g^{2} \int_{-\infty}^{+\infty} d z_{1 *} \int_{-\infty}^{z_{1 *}} d z_{2 *}\left[-\infty p_{1}, z_{2 *}\right]_{x}^{b c} \bar{\psi}\left(z_{2 *}\right) \gamma^{5} \not p_{1} t^{c}\left[z_{2 *}, z_{1 *}\right]_{x} t^{d} \psi\left(z_{1 *}\right)\left[z_{1 *}, \infty p_{1}\right]_{x}^{d a}\right\rangle \\
& \quad=-g^{2} \int_{0}^{+\infty} d z_{1 *} \int_{-\infty}^{0} d z_{2 *}\left(t^{b} U_{x}^{\dagger} t^{a}\right)_{i j} \operatorname{tr}\left\{\gamma^{5} \not p_{1}\left\langle\psi_{j}\left(z_{1 *}, x_{\perp}\right) \bar{\psi}_{i}\left(z_{2 *}, x_{\perp}\right)\right\rangle\right\}
\end{aligned}
$$

we now need quark propagator (3.2) and proceeding as before we integrate over the longitudinal coordinates $z_{1 *}$ and $z_{2 *}$ and perform the Fourier transform we arrive at

$$
\begin{aligned}
& \left\langle\mathcal{Q}_{5 x}^{a b}\right\rangle_{\text {Figure 16b }} \\
& =-g^{2}\left(t^{b} U_{x}^{\dagger} t^{a}\right)_{i j} \int_{0}^{+\infty} d z_{1 *} \int_{-\infty}^{0} d z_{2 *} \operatorname{tr}\left\{\gamma^{5} \not p_{1} \int_{0}^{+\infty} \frac{d \alpha}{2 \alpha} \frac{1}{\alpha s}\left\langle x_{\perp}\left|e^{-i \frac{p_{\perp}^{2}}{\alpha s} z_{1 *}}\left(\alpha \not p_{1}+\not p_{\perp}\right)\right| z_{\perp}\right\rangle \not p_{2} \gamma^{5}\right. \\
& \times \frac{i g}{4 \alpha} \int_{-\infty}^{+\infty} d \frac{2}{s} z_{*}\left[\infty p_{1}, z_{*}\right]_{z} \epsilon^{i j} F_{i j}\left[z_{*},-\infty p_{1}\right]_{z}\left\langle z_{\perp}\left|\left(\alpha p_{1}+\not p_{\perp}\right) e^{i \frac{p_{\perp}^{2}}{\alpha s} z_{2 *}}\right| x_{\perp}\right\rangle \\
& =\frac{g^{2}}{8 \pi} \int_{0}^{+\infty} \frac{d \alpha}{\alpha} \operatorname{tr}\left\{\gamma^{5} \not p_{1} \frac{i(\not p-\not \hbar)_{\perp}}{2 \pi(x-z)_{\perp}^{2}} \not p_{2} \gamma^{5} \frac{i(\not-\not x)_{\perp}}{2 \pi(x-z)_{\perp}^{2}}\right\} \\
& \times i g \int_{-\infty}^{+\infty} d z_{*}\left(\left[\infty p_{1}, z_{*}\right]_{z} \epsilon^{i j} F_{i j}\left[z_{*},-\infty p_{1}\right]_{z}\right)_{i j}\left(t^{b} U_{x}^{\dagger} t^{a}\right)_{i j} \\
& =-\frac{\alpha_{s}}{2 \pi^{2}} \int_{0}^{+\infty} \frac{d \alpha}{\alpha} \int \frac{d^{2} z}{(x-z)_{\perp}^{2}} g \int_{-\infty}^{+\infty} d z_{*}\left(\left[\infty p_{1}, z_{*}\right]_{z} \epsilon^{i j} i \frac{s}{2} F_{i j}\left[z_{*},-\infty p_{1}\right]_{z}\right)_{i j}\left(t^{b} U_{x}^{\dagger} t^{a}\right)_{i j} \\
& =-\frac{\alpha_{s}}{2 \pi^{2}} \int_{0}^{+\infty} \frac{d \alpha}{\alpha} \int \frac{d^{2} z}{(x-z)_{\perp}^{2}} \operatorname{Tr}\left\{\mathcal{F}_{z} t^{b} U_{x}^{\dagger} t^{a}\right\}
\end{aligned}
$$

where we used $\operatorname{tr}\left\{\gamma^{5} \not p_{2}(\not x-\not)_{\perp} \not p_{2} \gamma^{5}(\not k-\not x)_{\perp}\right\}=2 s(x-z)_{\perp}^{2}$ and definition of operator $\hat{\mathcal{F}}_{x}$, eq. (3.6).

Next, we consider $\mathcal{Q}_{1 x}^{a b}$ and proceed in the same way

$$
\begin{aligned}
\left\langle\mathcal{Q}_{1 x}^{a b}\right\rangle_{\text {Figure } 16 b}= & \frac{g^{2}}{8 \pi} \int_{0}^{+\infty} \frac{d \alpha}{\alpha} \operatorname{tr}\left\{i \not p_{1} \frac{i(\not x-\not)_{\perp}}{2 \pi(x-z)_{\perp}^{2}} \not p_{2} \gamma^{5} \frac{i(\not-\not x)_{\perp}}{2 \pi(x-z)_{\perp}^{2}}\right\} \\
& \times i g \int_{-\infty}^{+\infty} d z_{*}\left(\left[\infty p_{1}, z_{*}\right]_{z} \epsilon^{i j} F_{i j}\left[z_{*},-\infty p_{1}\right]_{z}\right)_{i j}\left(t^{b} U_{x}^{\dagger} t^{a}\right)_{i j}=0
\end{aligned}
$$

where we used $\operatorname{tr}\left\{i \not p_{1}(\not x-\not)_{\perp} \not p_{2} \gamma^{5}(\not z-\not x)_{\perp}\right\}=0$, so we find that the quark operator $\mathcal{Q}_{1}^{a b}$ does not mix with the gluon operator $\mathcal{F}$ under one loop evolution. We have obtained the same result for the quark operator $\mathcal{Q}_{1}$ defined in (4.9).

Summing up diagrams in figure $16 \mathrm{a}$ and b for operator $\mathcal{Q}_{5 x}^{a b}{ }^{\dagger}$, we have

$$
\left\langle\mathcal{Q}_{5 x}^{a b}\right\rangle_{\text {Figure } 16}=-\frac{\alpha_{s}}{2 \pi^{2}} \int_{0}^{+\infty} \frac{d \alpha}{\alpha} \int \frac{d^{2} z}{(x-z)_{\perp}^{2}} \operatorname{Tr}\left\{\left(\mathcal{F}_{z}+\mathcal{Q}_{5 z}\right) t^{b} U_{x}^{\dagger} t^{a}\right\}
$$


from which we can easily deduce

$$
\left\langle\mathcal{Q}_{5 x}^{a b}\right\rangle_{\text {Figure 16 }}=-\frac{\alpha_{s}}{2 \pi^{2}} \int_{0}^{+\infty} \frac{d \alpha}{\alpha} \int \frac{d^{2} z}{(x-z)_{\perp}^{2}} \operatorname{Tr}\left\{\left(\mathcal{F}_{z}^{\dagger}+\mathcal{Q}_{5 z}^{\dagger}\right) t^{a} U_{x} t^{b}\right\} .
$$

The evolution of operator $\operatorname{Tr}_{\mathrm{a}}\left\{U_{y}^{\dagger} \mathcal{F}_{x}\right\}$, diagrams in figure 9 and figure 10 can be deduced from $\operatorname{Tr}\left\{U_{y}^{\dagger} \mathcal{F}_{x}\right\}$, by changing $t^{a} \rightarrow T^{a}$ and $\operatorname{Tr} \rightarrow \operatorname{Tr}_{\mathrm{a}}$ in eq. (6.49).

We can conclude that the evolution of $\operatorname{Tr}_{\mathrm{a}}\left\{U_{y}^{\dagger}\left(\mathcal{F}_{x}+\mathcal{Q}_{5 x}\right)\right\}$, excluding the BK-type diagrams, is

$$
\begin{aligned}
&\left\langle\operatorname{Tr}_{\mathrm{a}}\left\{U_{y}^{\dagger}\left(\mathcal{F}_{x}+\mathcal{Q}_{5 x}\right)\right\}\right\rangle_{\text {Figures }} 9+10+16 \\
&=-\frac{\alpha_{s}}{2 \pi^{2}} \int_{0}^{+\infty} \frac{d \alpha}{\alpha} \int d^{2} z\left\{\frac{1}{(x-z)_{\perp}^{2}} \operatorname{Tr}\left\{\left(\mathcal{F}_{z}^{\dagger}+\mathcal{Q}_{5 z}^{\dagger}\right) t^{a} U_{x} t^{b}\right\} U_{y}^{a b}\right. \\
&+2 \operatorname{Tr}_{\mathrm{a}}\left\{U_{x} T^{a} U_{y}^{\dagger} T^{b}\right\}\left[\frac{(\vec{x}-\vec{z}) \times(\vec{z}-\vec{y})}{(x-z)_{\perp}^{2}(y-z)_{\perp}^{2}}\left(\mathcal{Q}_{1 z}^{b a}-\mathcal{Q}_{1 z}^{b a \dagger}\right)\right. \\
&-\left(\frac{(x-z, z-y)}{(x-z)_{\perp}^{2}(y-z)_{\perp}^{2}}+\frac{1}{(x-z)_{\perp}^{2}}\right)\left(\mathcal{Q}_{5 z}^{b a}+\mathcal{Q}_{5 z}^{b a \dagger}+\mathcal{F}_{z}^{b a}\right) \\
&\left.\left.-4 \pi^{2} \int d^{2} q_{1} \frac{e^{i\left(q_{1}, y-z\right)}-e^{i\left(q_{1}, x-z\right)}}{q_{1 \perp}^{2}} \delta^{(2)}(z-x) \mathcal{F}_{z}^{b a}\right]\right\}
\end{aligned}
$$

Note, that in the r.h.s. we have trace in the adjoint representation, while in the l.h.s. we have trace in the fundamental representation in the first term, and trace in the adjoint representation in the second term. In eq. (F.45) we have mixing with operator $\hat{\mathcal{Q}}_{1}\left(x_{\perp}\right)$ which may contribute to spin in the non forward case. Evolution equation (F.45) agrees with the one calculated in refs. [7, 8] except for the mixing term proportional to $\hat{\mathcal{Q}}_{1}\left(x_{\perp}\right)$. However, when we perform the color algebra, the evolution equation (F.45) is entirely written in terms of operators $\hat{Q}_{1}\left(x_{\perp}\right) \hat{Q}_{5}\left(x_{\perp}\right), \hat{\tilde{Q}}_{1}\left(x_{\perp}\right)$, and $\hat{\tilde{Q}}_{5}\left(x_{\perp}\right)$.

Open Access. This article is distributed under the terms of the Creative Commons Attribution License (CC-BY 4.0), which permits any use, distribution and reproduction in any medium, provided the original author(s) and source are credited.

\section{References}

[1] D. Boer et al., Gluons and the quark sea at high energies: distributions, polarization, tomography, arXiv:1108.1713 [INSPIRE].

[2] NNPDF collaboration, A first unbiased global determination of polarized PDFs and their uncertainties, Nucl. Phys. B 887 (2014) 276 [arXiv:1406.5539] [INSPIRE].

[3] D. de Florian, R. Sassot, M. Stratmann and W. Vogelsang, Global analysis of helicity parton densities and their uncertainties, Phys. Rev. Lett. 101 (2008) 072001 [arXiv:0804.0422] [INSPIRE].

[4] D. de Florian, R. Sassot, M. Stratmann and W. Vogelsang, Extraction of spin-dependent parton densities and their uncertainties, Phys. Rev. D 80 (2009) 034030 [arXiv:0904.3821] [INSPIRE]. 
[5] Y.V. Kovchegov, D. Pitonyak and M.D. Sievert, Helicity evolution at small-x, JHEP 01 (2016) 072 [Erratum ibid. 10 (2016) 148] [arXiv: 1511.06737] [INSPIRE].

[6] Y.V. Kovchegov, D. Pitonyak and M.D. Sievert, Small-x asymptotics of the gluon helicity distribution, JHEP 10 (2017) 198 [arXiv:1706.04236] [INSPIRE].

[7] Y.V. Kovchegov, D. Pitonyak and M.D. Sievert, Helicity evolution at small-x: flavor singlet and non-singlet observables, Phys. Rev. D 95 (2017) 014033 [arXiv:1610.06197] [INSPIRE].

[8] Y.V. Kovchegov and M.D. Sievert, Small-x helicity evolution: an operator treatment, Phys. Rev. D 99 (2019) 054032 [arXiv: 1808.09010] [inSPIRE].

[9] R. Boussarie, Y. Hatta and F. Yuan, Proton spin structure at small-x, Phys. Lett. B $\mathbf{7 9 7}$ (2019) 134817 [arXiv:1904.02693] [INSPIRE].

[10] A. Tarasov and R. Venugopalan, Role of the chiral anomaly in polarized deeply inelastic scattering: finding the triangle graph inside the box diagram in Bjorken and Regge asymptotics, Phys. Rev. D 102 (2020) 114022 [arXiv:2008.08104] [InSPIRE].

[11] Y. Hatta, Y. Nakagawa, F. Yuan, Y. Zhao and B. Xiao, Gluon orbital angular momentum at small-x, Phys. Rev. D 95 (2017) 114032 [arXiv:1612.02445] [INSPIRE].

[12] Y. Hatta, Nucleon electric dipole moment from polarized deep inelastic scattering, Phys. Lett. B $\mathbf{8 1 4}$ (2021) 136126 [arXiv:2012.01865] [INSPIRE].

[13] Y. Hatta, CP-odd gluonic operators in QCD spin physics, Phys. Rev. D 102 (2020) 094004 [arXiv:2009.03657] [INSPIRE].

[14] E.A. Kuraev, L.N. Lipatov and V.S. Fadin, The Pomeranchuk singularity in non-Abelian gauge theories, Sov. Phys. JETP 45 (1977) 199 [Zh. Eksp. Teor. Fiz. 72 (1977) 377] [INSPIRE].

[15] I.I. Balitsky and L.N. Lipatov, The Pomeranchuk singularity in quantum chromodynamics, Sov. J. Nucl. Phys. 28 (1978) 822 [Yad. Fiz. 28 (1978) 1597] [INSPIRE].

[16] H1 and ZEUS collaborations, Combined measurement and $Q C D$ analysis of the inclusive $e^{ \pm} p$ scattering cross sections at HERA, JHEP 01 (2010) 109 [arXiv:0911.0884] [INSPIRE].

[17] H1 and ZEUS collaborations, Combination of measurements of inclusive deep inelastic $e^{ \pm} p$ scattering cross sections and QCD analysis of HERA data, Eur. Phys. J. C $\mathbf{7 5}$ (2015) 580 [arXiv: 1506.06042] [INSPIRE].

[18] I.I. Balitsky and V.M. Braun, Evolution equations for QCD string operators, Nucl. Phys. B 311 (1989) 541 [inSPIRE].

[19] I. Balitsky, Operator expansion for high-energy scattering, Nucl. Phys. B 463 (1996) 99 [hep-ph/9509348] [INSPIRE].

[20] I. Balitsky and G.A. Chirilli, Next-to-leading order evolution of color dipoles, Phys. Rev. D 77 (2008) 014019 [arXiv:0710.4330] [INSPIRE].

[21] I. Balitsky and G.A. Chirilli, NLO evolution of color dipoles in $N=4 S Y M$, Nucl. Phys. B $\mathbf{8 2 2}$ (2009) 45 [arXiv:0903.5326] [INSPIRE].

[22] I. Balitsky and G.A. Chirilli, Photon impact factor in the next-to-leading order, Phys. Rev. D 83 (2011) 031502 [arXiv: 1009.4729] [INSPIRE].

[23] I. Balitsky and G.A. Chirilli, Photon impact factor and $k_{T}$-factorization for DIS in the next-to-leading order, Phys. Rev. D 87 (2013) 014013 [arXiv:1207.3844] [INSPIRE]. 
[24] J. Jalilian-Marian, A. Kovner, A. Leonidov and H. Weigert, The Wilson renormalization group for low $x$ physics: towards the high density regime, Phys. Rev. D 59 (1998) 014014 [hep-ph/9706377] [INSPIRE].

[25] E. Ferreiro, E. Iancu, A. Leonidov and L. McLerran, Nonlinear gluon evolution in the color glass condensate. 2, Nucl. Phys. A 703 (2002) 489 [hep-ph/0109115] [INSPIRE].

[26] E. Iancu, A. Leonidov and L.D. McLerran, Nonlinear gluon evolution in the color glass condensate. 1, Nucl. Phys. A 692 (2001) 583 [hep-ph/0011241] [INSPIRE].

[27] Y.V. Kovchegov, Small-x $F_{2}$ structure function of a nucleus including multiple Pomeron exchanges, Phys. Rev. D 60 (1999) 034008 [hep-ph/9901281] [INSPIRE].

[28] Y.V. Kovchegov, Unitarization of the BFKL Pomeron on a nucleus, Phys. Rev. D 61 (2000) 074018 [hep-ph/9905214] [INSPIRE].

[29] I. Balitsky, High-energy QCD and Wilson lines, hep-ph/0101042 [INSPIRE].

[30] Y.V. Kovchegov and E. Levin, Quantum chromodynamics at high energy, Cambridge University Press, Cambridge, U.K. (2012) [INSPIRE].

[31] V.S. Fadin and L.N. Lipatov, BFKL Pomeron in the next-to-leading approximation, Phys. Lett. B 429 (1998) 127 [hep-ph/9802290] [INSPIRE].

[32] G. Beuf, Dipole factorization for DIS at NLO: combining the $q \bar{q}$ and $q \bar{q} g$ contributions, Phys. Rev. D 96 (2017) 074033 [arXiv:1708.06557] [InSPIRE].

[33] G. Beuf, H. Hänninen, T. Lappi and H. Mäntysaari, Color glass condensate at next-to-leading order meets HERA data, Phys. Rev. D 102 (2020) 074028 [arXiv: 2007.01645] [INSPIRE].

[34] R. Kirschner and L.n. Lipatov, Double logarithmic asymptotics and Regge singularities of quark amplitudes with flavor exchange, Nucl. Phys. B 213 (1983) 122 [INSPIRE].

[35] B.I. Ermolaev, S.I. Manaenkov and M.G. Ryskin, Nonsinglet structure functions at small $x$, Z. Phys. C 69 (1996) 259 [hep-ph/9502262] [InSPIRE].

[36] J. Bartels, B.I. Ermolaev and M.G. Ryskin, Nonsinglet contributions to the structure function $g_{1}$ at small $x, Z$. Phys. C $\mathbf{7 0}$ (1996) 273 [hep-ph/9507271] [INSPIRE].

[37] J. Bartels, B.I. Ermolaev and M.G. Ryskin, Flavor singlet contribution to the structure function $g_{1}$ at small $x, Z$. Phys. C 72 (1996) 627 [hep-ph/9603204] [INSPIRE].

[38] T. Jaroszewicz, Gluonic Regge singularities and anomalous dimensions in QCD, Phys. Lett. B 116 (1982) 291 [INSPIRE].

[39] I. Balitsky, NLO BFKL and anomalous dimensions of light-ray operators, Int. J. Mod. Phys. Conf. Ser. 25 (2014) 1460024 [INSPIRE].

[40] G. Altarelli and G. Parisi, Asymptotic freedom in parton language, Nucl. Phys. B 126 (1977) 298 [INSPIRE].

[41] R. Mertig and W.L. van Neerven, The calculation of the two loop spin splitting functions $P_{i j}^{(1)}(x)$, Z. Phys. C 70 (1996) 637 [hep-ph/9506451] [INSPIRE].

[42] S. Moch, J.A.M. Vermaseren and A. Vogt, The three-loop splitting functions in QCD: the helicity-dependent case, Nucl. Phys. B 889 (2014) 351 [arXiv:1409.5131] [INSPIRE].

[43] A. Behring et al., The polarized three-loop anomalous dimensions from on-shell massive operator matrix elements, Nucl. Phys. B 948 (2019) 114753 [arXiv:1908.03779] [INSPIRE]. 
[44] J. Blumlein and A. Vogt, On the behavior of nonsinglet structure functions at small $x$, Phys. Lett. B 370 (1996) 149 [hep-ph/9510410] [INSPIRE].

[45] J. Blumlein and A. Vogt, The singlet contribution to the structure function $g_{1}\left(x, Q^{2}\right)$ at small x, Phys. Lett. B 386 (1996) 350 [hep-ph/9606254] [INSPIRE].

[46] G.A. Chirilli, Sub-eikonal corrections to scattering amplitudes at high energy, JHEP 01 (2019) 118 [arXiv:1807.11435] [INSPIRE].

[47] I. Balitsky and A. Tarasov, Rapidity evolution of gluon TMD from low to moderate $x$, JHEP 10 (2015) 017 [arXiv:1505.02151] [INSPIRE].

[48] I. Balitsky and A. Tarasov, Gluon TMD in particle production from low to moderate $x$, JHEP 06 (2016) 164 [arXiv: 1603.06548] [InSPIRE].

[49] D.W. Sivers, Single spin production asymmetries from the hard scattering of point-like constituents, Phys. Rev. D 41 (1990) 83 [InSPIRE].

[50] D.W. Sivers, Hard scattering scaling laws for single spin production asymmetries, Phys. Rev. D 43 (1991) 261 [INSPIRE].

[51] P.J. Mulders and R.D. Tangerman, The complete tree level result up to order $1 / Q$ for polarized deep inelastic leptoproduction, Nucl. Phys. B 461 (1996) 197 [Erratum ibid. 484 (1997) 538] [hep-ph/9510301] [INSPIRE].

[52] K. Goeke, A. Metz and M. Schlegel, Parameterization of the quark-quark correlator of a spin-1/2 hadron, Phys. Lett. B 618 (2005) 90 [hep-ph/0504130] [INSPIRE].

[53] A. Bacchetta, M. Diehl, K. Goeke, A. Metz, P.J. Mulders and M. Schlegel, Semi-inclusive deep inelastic scattering at small transverse momentum, JHEP 02 (2007) 093 [hep-ph/0611265] [INSPIRE].

[54] Y.V. Kovchegov, Orbital angular momentum at small x, JHEP 03 (2019) 174 [arXiv: 1901.07453] [INSPIRE].

[55] J.C. Collins, Foundations of perturbative QCD, Cambridge University Press, Cambridge, U.K. (2011).

[56] T. Altinoluk, N. Armesto, G. Beuf, M. Martínez and C.A. Salgado, Next-to-eikonal corrections in the CGC: gluon production and spin asymmetries in $p A$ collisions, JHEP 07 (2014) 068 [arXiv: 1404.2219] [INSPIRE].

[57] T. Altinoluk, N. Armesto, G. Beuf and A. Moscoso, Next-to-next-to-eikonal corrections in the CGC, JHEP 01 (2016) 114 [arXiv: 1505.01400] [INSPIRE].

[58] T. Altinoluk, G. Beuf, A. Czajka and A. Tymowska, Quarks at next-to-eikonal accuracy in the CGC I: forward quark-nucleus scattering, arXiv:2012.03886 [INSPIRE]. 